University of Louisville

ThinkIR: The University of Louisville's Institutional Repository

Electronic Theses and Dissertations

$12-2020$

\title{
Design, synthesis and application of Janus gold nanoprisms for directed self-assembly.
}

Md. Emtias Chowdhury

University of Louisville

Follow this and additional works at: https://ir.library.louisville.edu/etd

Part of the Analytical Chemistry Commons, and the Materials Chemistry Commons

\section{Recommended Citation}

Chowdhury, Md. Emtias, "Design, synthesis and application of Janus gold nanoprisms for directed selfassembly." (2020). Electronic Theses and Dissertations. Paper 3539.

https://doi.org/10.18297/etd/3539

This Doctoral Dissertation is brought to you for free and open access by ThinkIR: The University of Louisville's Institutional Repository. It has been accepted for inclusion in Electronic Theses and Dissertations by an authorized administrator of ThinkIR: The University of Louisville's Institutional Repository. This title appears here courtesy of the author, who has retained all other copyrights. For more information, please contact thinkir@louisville.edu. 


\title{
DESIGN, SYNTHESIS AND APPLICATION OF JANUS GOLD NANOPRISMS FOR DIRECTED SELF-
}

\author{
ASSEMBLY \\ By \\ Md. Emtias Chowdhury \\ A Dissertation \\ Submitted to the Faculty of the \\ College of Arts and Sciences of the University of Louisville \\ in Partial Fulfillment of the Requirements \\ for the Degree of
}

Doctor of Philosophy in Chemistry

Department of Chemistry

University of Louisville

Louisville, Kentucky

December 2020 



\title{
DESIGN, SYNTHESIS AND APPLICATION OF JANUS GOLD NANOPRISMS FOR DIRECTED SELF- ASSEMBLY
}

\author{
By \\ Md. Emtias Chowdhury
}

A Dissertation Approved on

November 23, 2020

by the following Dissertation Committee:

Dissertation Director: Dr. Martin G. O’Toole

$\overline{\text { Dissertation Co-director: Dr. Craig A. Grapperhaus }}$

Dr. Francis P. Zamborini

Dr. Muriel C. Maurer

Dr. Stuart Williams 


\title{
DEDICATION
}

Dedicated to my parents, Anowara and Elias

\author{
and my beloved wife, Nezahat
}




\section{ACKNOWLEDGEMENT}

I would like to take this opportunity to express my gratitude to everyone who supported me throughout the long and arduous journey towards my PhD. First and foremost, I would first like to express my sincere gratitude and indebtedness to my advisor Dr. Martin O'Toole for his continual support, motivation, and guidance throughout my $\mathrm{PhD}$ program. I really appreciate him giving me an enormous amount freedom, which allowed me to design my own experiments and think about my own works in a critical way. He also motivated me to dive deep into literatures that instilled an ability of ideation in me. I also appreciate his patience with my efforts. Without his assistance, my thesis writing wouldn't have been completed.

I would like to thank my dissertation co-advisor Dr. Craig Grapperhaus for his valuable discussions and suggestions, which helped me greatly to do my research. I would also like to thank my committee members Dr. Francis P. Zamborini, Dr. Muriel C. Maurer, and Dr. Stuart Williams for their questions, feedbacks, and suggestions on my research during my "original research proposal (ORP)" presentation, and compliments during my 'research seminar' for the progress I was able to make 9 months after my ORP, all of which were extremely helpful and significantly shaped my research. 
My sincere thanks goes to Kurtis James, who not only taught me nanoprisms synthesis, SEM, Dark field microscopy and so many other things, but also helped me become acclimatized in the lab and get things going from the very beginning. I must also thank our lab manager Betty Nun for always extending a helping hand to me. Every time I needed a chemical, either she promptly ordered it if possible or helped me find it in the lab. Quite a few times, she even brought chemicals from other labs for me.

There are few other members of our research group I would like to thank, who helped me with my research in many ways and supported me during challenging times. I would like to thank Dr. Rajat Chauhan for his insightful discussions about research and helping me with CMCS-curcumin biopolymer synthesis. Rajat and I are from the same region (South Asian), which has made our daily conversation about various societal aspects more interesting and refreshing. I would also like to thank Nick and Nagwa for their support.

I also have to thank Shahinur Rahman from the lab next to ours for collaborating with me and helping me with Raman experiments, , and Anwar for providing me a special 'Vial', which essentially helped me solve my long-standing problems in getting the DNA melting curve.

I would like to express my gratitude to my high school teacher, Mr. Iqbal for teaching me math and science in an interesting and at the same time the most understandable way, thereby strongly encouraging me to pursue a science career. 
I am extremely grateful to my parents for their unending support and love, guidance, and opportunities that they provided me. Without their inspiration and guidance, I certainly would not be where I am today.

Last but not least, I am grateful to my lovely wife for her unconditional love and unflinching support and inspiration through thick and thin. 


\title{
ABSTRACT \\ DESIGN, SYNTHESIS AND APPLICATION OF JANUS GOLD NANOPRISMS FOR DIRECTED SELF- ASSEMBLY
}

\author{
Md. Emtias Chowdhury
}

November 23, 2020

Colloidal Janus particles that possess more than one type of surface chemistry or functionalities have drawn significant interest due to their enormous potential in bottom-up synthetic strategies for complex superstructures. Moreover, the property of molecular recognition, tunability, and predictability of the DNA-mediated interactions enable a high degree of control over particle assembly to generate highly ordered nanostructures with emergent applications. In this dissertation, we present our works on the synthesis of Janus particles from anisotropic gold nanoprisms, and DNAmediated assembly of nanoprisms and polymer beads in four major areas: 1) Facet selective asymmetric functionalization of gold nanoprisms for Janus particle synthesis, 2) Synthesis and plasmonic properties of nanoprism dimers and trimers, 3) DNA mediated hierarchical organization of gold nanoprisms into 3D superlattices 
and their application in Surface-enhanced Raman scattering (SERS) and Fluorescence, and 4) DNA induced assembly of nanoprisms and polystyrene beads into 3D SERS substrates.

The first set of Janus particles, consisting of gold nanoprisms coated with hexadecane and DNA dynamically align themselves at the interface of a waterchloroform mixture. The Janus character of a second set of Janus particles functionalized with PEG and DNA is confirmed by the selective attachment of gold nanoparticles to the DNA-containing facets of Janus nanoprisms. The presence of two mutually exclusive coatings on the opposite major facets of nanoprisms allows the formation of nanoprisms dimers and trimers, which exhibit distinctly different plasmonic properties from their monomer counterparts.

Furthermore, anisotropic nanoparticles associate in a manner that ensures maximum hybridization interactions. DNA-induced and shape directed face-to-face assembly of anisotropic gold nanoprisms leads to the formation of nanoprism 1D stacks, which are then assembled into 3D nanoprism superlattices using a near- $\mathrm{T}_{\mathrm{m}}$ thermal annealing approach that promotes long-range DNA-induced interaction and ordering.

Finally, we fabricate a large area SERS substate via a two-step DNA mediated assembly of gold nanoprisms and polystyrene beads into a large ensemble of beads, which consist of 20-50 nanoprism-coated beads. An excellent enhancement factor (EF) of $1.09 \times 10^{5}$ and a very high detection sensitivity (up to $10^{-10} \mathrm{M}$ ) are observed for the analysis of a probe molecule (Methylene blue) using the SERS substrate. 


\section{TABLE OF CONTENT}

ACKNOWLEDGEMENT ................................................................ IV

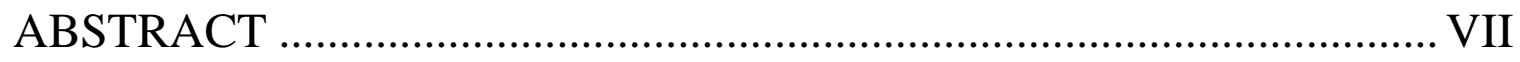

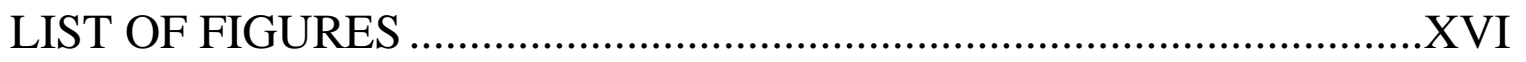

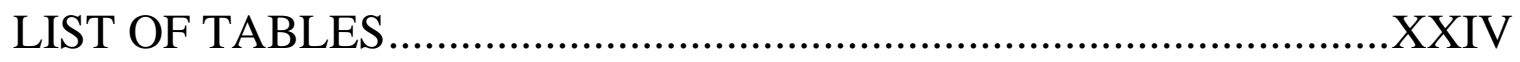

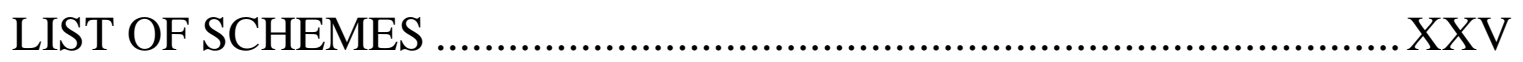

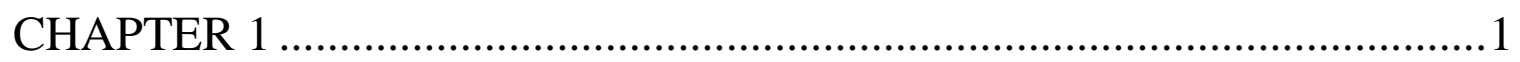

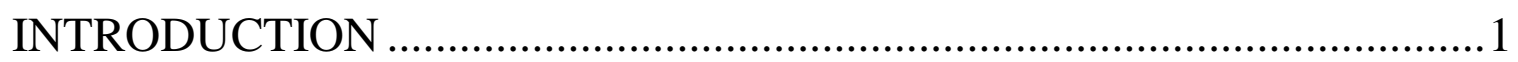

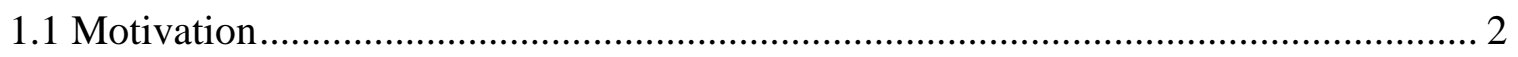

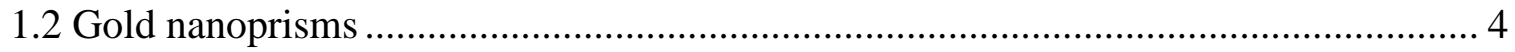

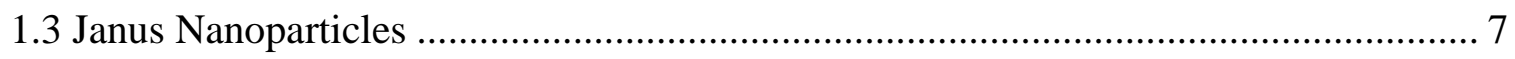

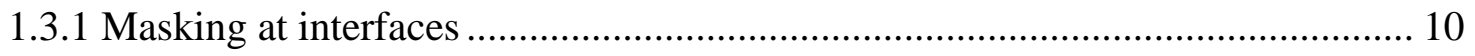

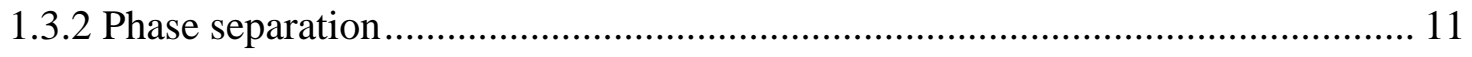


1.4 DNA-mediated assembly of nanoparticles .......................................................... 14

1.4.1 DNA-mediated assembly of spherical nanoparticles .......................................... 16

1.4.2 DNA-mediated assembly of anisotropic particles .......................................... 19

1.5 Metal nanoparticle coated polymer beads........................................................... 21

1.6 Optical properties of DNA guided gold nanostructures ........................................... 25

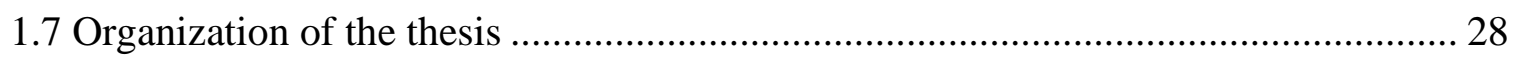

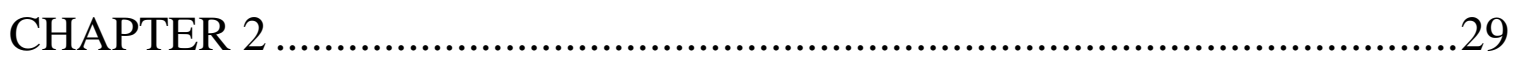

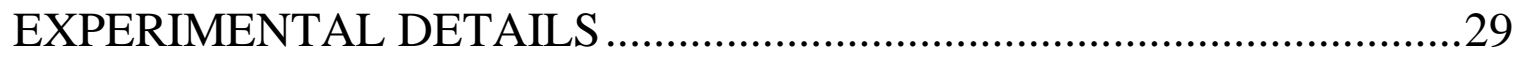

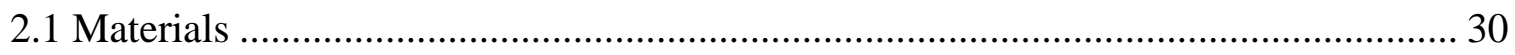

2.2 List of oligonucleotide linkers used in this study .................................................... 31

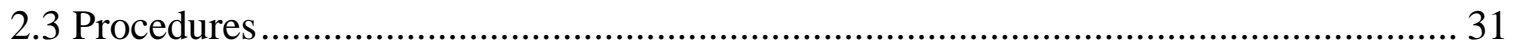

2.3.1 Chemical synthesis and purification of gold triangular nanoprisms.................... 31

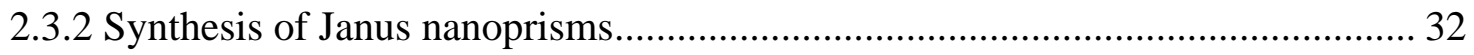

2.3.3 Functionalization of nanoprisms with oligonucleotide........................................ 34

2.3.4 Functionalization of nanoprisms with 1-Hexadecane thiol ................................. 34

2.3.5 Functionalization of nanoprisms with thiol modified polyethylene glycol (PEG-

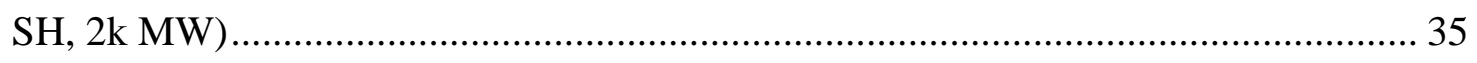


2.3.6 Selective attachment of gold nanospheres to major facets of gold nanoprisms .. 35

2.3.7 Functionalization of glass slides with Amino groups with APTES................... 36

2.3.8 Functionalization of glass slides with Poly(siloxanes) .................................. 36

2.3.9 Synthesis of $20 \mathrm{~nm}$ average diameter citrate-stabilized gold nanospheres ......... 36

2.3.10 Functionalization of $20 \mathrm{~nm}$ gold nanospheres with oligonucleotides............... 37

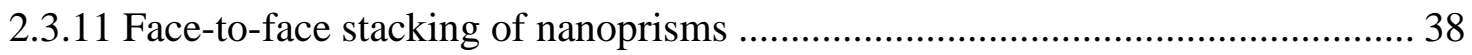

2.3.12 Melting analysis of $1 \mathrm{D}$ nanoprisms stacks................................................ 39

2.3.13 Formation of 3D nanoprism superlattices .............................................. 40

2.3.14 Surface modification of carboxylate modified polystyrene (PS) beads............ 40

2.3.15 Coating of gold nanoprisms onto PS beads ............................................ 45

2.3.16 Melting analysis of PS bead/gold nanoprism conjugates ............................. 45

2.3.17 Formation of 3D PS beads/gold nanoprism aggregates ............................... 46

2.3.18 Synthesis of gold nanoprism dimers and trimers ................................... 46

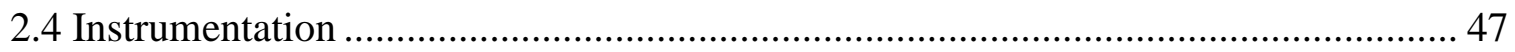

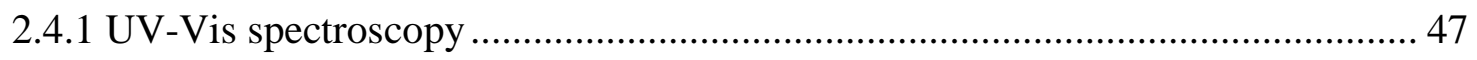

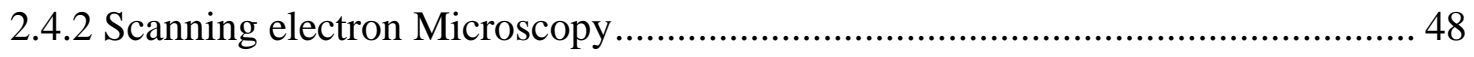

2.4.3 Dynamic Light Scattering (DLS) size and zeta potential measurements ........... 49

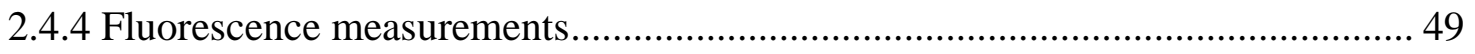

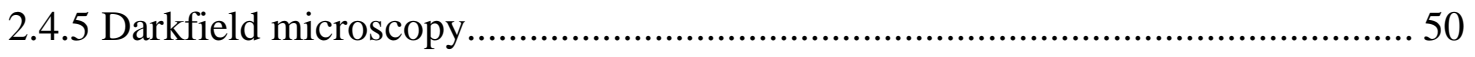

2.4.6 Acquisition of single particle scattering profiles ...................................... 50

2.4.7 Surface Enhanced Raman Scattering Experiments ........................................ 51

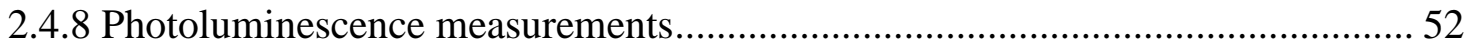


FACET SELECTIVE ASYMMETRIC FUNCTIONALIZATION OF ANISOTROPIC GOLD NANOPRISMS FOR JANUS PARTICLE SYNTHESIS

3.1 Introduction 54

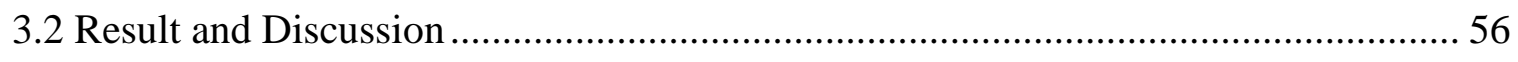

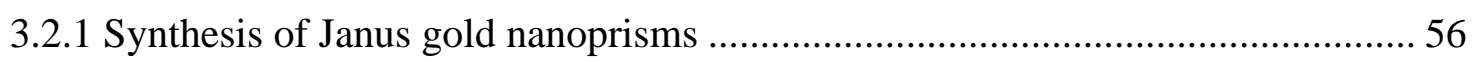

3.2.2 Characterization of Janus nanoprisms ....................................................... 58

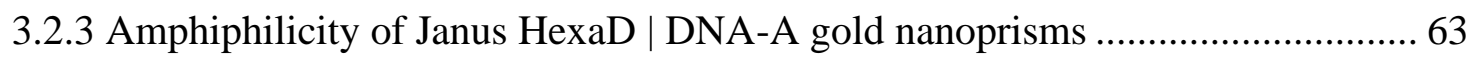

3.2.4 Selective adsorption of Janus nanoprisms on both hydrophilic and hydrophobic

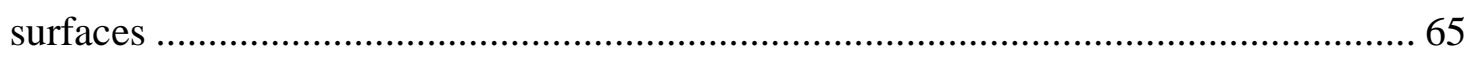

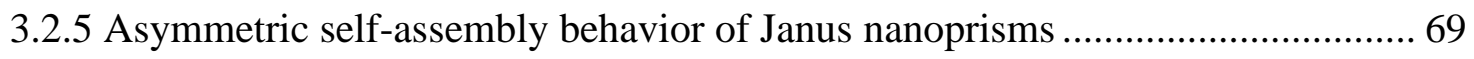

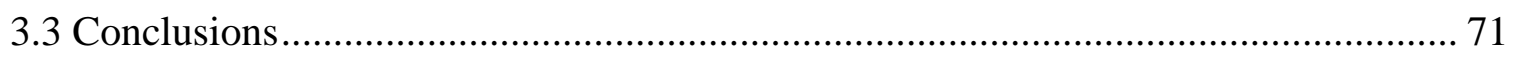

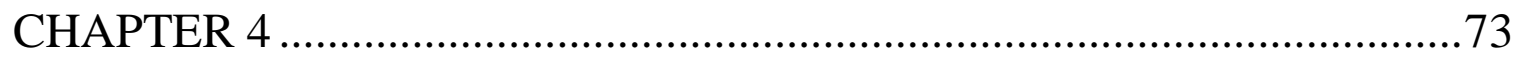

DNA-MEDIATED HIERARCHICAL ORGANIZATION OF GOLD

NANOPRISMS INTO 3D SUPERLATTICES FOR SURFACE-

ENHANCED RAMAN SCATTERING AND FLUORESCENCE.............73

4.1 Introduction 


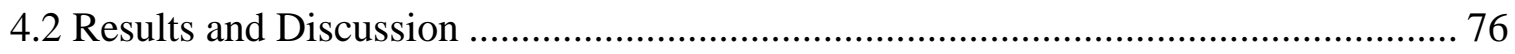

4.2.1 Face-to-face assembly of gold nanoprisms .................................................. 76

4.2.2 Long range ordering of nanoprisms into 3D superlattices .................................. 79

4.2.3 Surface enhanced Raman scattering (SERS) of 3D nanoprism superlattices ...... 81

4.2.4 Surface enhanced fluorescence spectroscopy of 3D superlattice …………….... 88

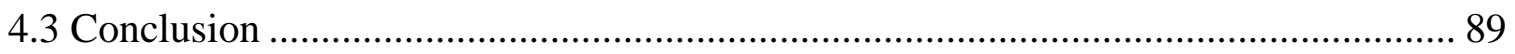

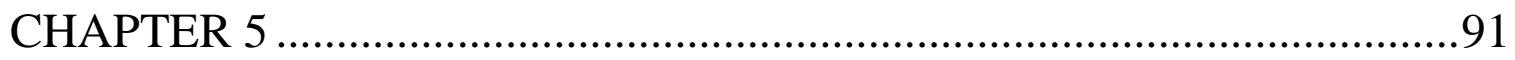

DNA-INDUCED ASSEMBLY OF GOLD NANOPRISMS AND

POLYSTYRENE BEADS INTO 3D PLASMONIC SERS SUBSTRATES

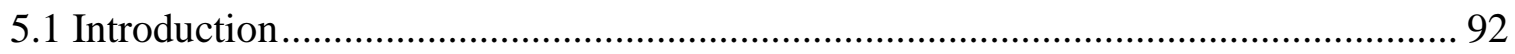

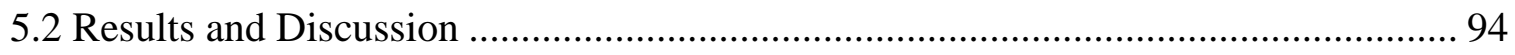

5.2.1 Conjugation of nanoprisms to carboxylate-modified PS beads ........................... 94

5.2.2 Characterization of PS beads/nanoprism conjugates .......................................... 96

5.2.3 Salt effects on the conjugation of nanoprisms onto PS beads ............................ 99

5.2.4 Fluorescence quenching of PS beads at different salt concentration................. 100

5.2.5 Formation of 3D PS beads/Nanoprism aggregates ............................................ 101

5.2.6 SERS activity of 3D PS beads/Nanoprism aggregates ...................................... 105

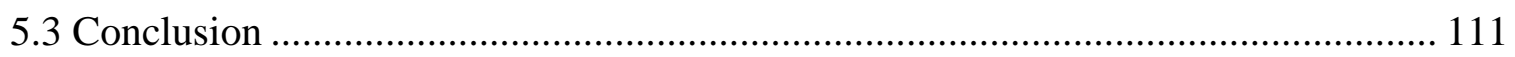




\section{SYNTHESIS AND OPTICAL PROPERTIES OF GOLD NANOPRISM}

DIMERS AND TRIMERS

6.1 Introduction

6.2 Results and discussion

6.2.1 Design and synthesis of gold nanoprism dimers and trimers

6.2.2 Characterization of Janus nanoprisms 118

6.2.3 Characterization of nanoprisms dimers and trimers 119

6.2.4 Plasmonic properties of single nanoprim dimers and trimers 124

6.3 Conclusion 128

CHAPTER 7

7.1 Summary and conclusions 130

7.2 Future Directions 135

REFERENCES 143

APPENDIX A 162 
SYNTHESIS, CHARACTERIZATION, AND ASYMMETRIC SURFACE FUNCTIONALIZATION OF GOLD NANOPRISMS 162

APPENDIX B 171

ADDITIONAL SEM AND DARKFIELD MICROSCOPY

CHARACTERIZATION OF NANOPRISM DIMERS, TRIMERS, SUPERLATTICES AND PS BEADS/ NANOPRISMS AGGREGATES.171

APPENDIX C

ADDITIONAL SERS SPECTRA OF METHYLENE BLUE AND PL SPECTRA OF ALEXA FLUOR PHALLOIDIN 647 ON 3D NANOPRISMS SUPERLATTICE AND 3D PS BEADS/ NANOPRISMS AGGREGATES. 193

CURRRICULUM VITAE .214 


\section{LIST OF FIGURES}

Figure 1.1: A) Dimensions of gold nanoprisms, B) Nanoprisms with sharp vertices,

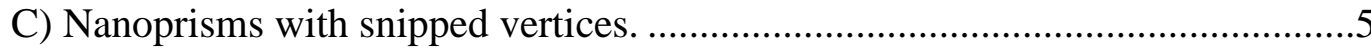

Figure 1.2: Three broad synthetic strategies for Janus particle synthesis....................9

Figure 2.1: Chemical structure of Sulfo-NHS ((N-hydroxysulfosuccinimide)..........42

Figure 2.2: Chemical structure of EDC (1-ethyl-3-(3-dimethylaminopropyl) carbodiimide).

Figure 3.1: UV-vis spectra of nanoprisms before and after purification.

Figure 3.2: Normalized UV-vis spectra of A) uncoated nanoprisms (blue), half HexaD coated (orange) and HexaD | DNA-A Janus nanoprisms (gray), and B) Uncoated nanoprisms (blue), half PEG coated (green), and PEG | DNA-A nanoprisms (red).

Figure 3.3: A) Representative Zeta Potential distribution graph of uncoated nanoprisms (red), HexaD | nanoprisms (Green), \& HexaD | DNA-A nanoprisms (blue), and B) representative Zeta Potential distribution graph of Uncoated nanoprisms (red), PEG | nanoprisms (Green), \& PEG | DNA-A nanoprisms (blue)

Figure 3.4: Amphiphilic Janus nanoprisms in a dual-phase water/chloroform system. Left: HexaD | HexaD nanoprisms, Middle: HexaD | DNA-A Janus, and Right: DNA-A | DNA-A nanoprisms.

Figure 3.5: Darkfield images of coated nanoprisms on hydrophilic and hydrophobic surface. (A-C) Deposition of DNA-A | DNA-A, HexaD | DNA-A Janus amphiphilic, and HexaD | HexaD nanoprisms on APTES respectively. (D-F) Deposition of DNA-A | DNA-A, HexaD | DNA-A amphiphilic Janus, and HexaD | HexaD nanoprisms on poly-siloxane, respectively. The insets show representative scattering spectra of the circled particles. 
Figure 3.6: Representative SEM images of coated nanoprisms on hydrophilic and hydrophobic surfaces. (A-C) Deposition of DNA-A | DNA-A, HexaD | DNA-A Janus amphiphilic, and HexaD | HexaD nanoprisms on APTES, respectively. (DF) Deposition of DNA-A | DNA-A, HexaD | DNA-A amphiphilic Janus, and HexaD | HexaD nanoprisms on poly-siloxane, respectively. The inset in $\mathrm{F}$ illustrates the formation of aggregates of HexaD-coated nanoprisms.

Figure 3.7: UV- Vis characterization of binding interactions between DNA-A" nanospheres and (A) PEG | PEG nanoprisms, (B) Janus PEG | DNA-A nanoprisms, and (C) DNA-A | DNA-A nanoprisms and representative SEM images of binding interactions between DNA-A" coated nanospheres (D\&G) PEG | PEG nanoprisms, (E\&H) Janus PEG | DNA-A nanoprisms, (F\&I) DNA-A | DNA-A nanoprisms. .70

Figure 4.1: DNA mediated Face-to-face assembly of gold nanoprisms. A, B, C) Representative SEM images of DNA mediated face-to-face assembly of nanoprisms into 1D stacks, D) Melting profile of 1D nanoprisms crystals, monitored at the SPR $\lambda_{\max }$ of nanoprisms. Inset shows the first derivative of the melting curve.

Figure 4.2: A) Melting profile of uncoated nanoprisms in DNA hybridization buffer, and B) SEM image of uncoated nanoprisms after mixing with DNA hybridization buffer.

Figure 4.3: DNA mediated 3-D hierarchical organization of gold nanoprisms. Dark field microscopy images of long-range ordering of 1D nanoprism stacks at A) Room temperature, B) $65^{\circ} \mathrm{C}$, C) $66^{\circ} \mathrm{C}$ and D) Just below $\mathrm{T}_{\mathrm{m}} \sim 68.5^{\circ} \mathrm{C}$. E, F) Representative low and high magnification SEM images of 3D nanoprisms superlattice formed below $\mathrm{T}_{\mathrm{m}}$, respectively.

Figure 4.4: A) Representative SEM image of 3-D hierarchical organization of gold nanoprosms on a glass slide. B) Raman spectrum of a pristine glass substrate (black) and 3D nanoprism superlattice (orange), and SERS spectra of $10^{-3} \mathrm{MB}$ adsorbed on glass substrate (green) and 3D nanoprism superlattices (dark red), 
and C) SERS spectra of MB on 3D nanoprism superlattices at different concentrations.

Figure 4.5: SERS spectra of $10^{-10} \mathrm{M} M B$ on 3D nanoprism superlattices. .84

Figure 4.6: A) Sample-to-sample SERS intensity variation at $1621 \mathrm{~cm}^{-1}$ recorded from 5 different substrates. Error bars represent standard deviations of SERS intensity at $1621 \mathrm{~cm}^{-1}$ from at least 6 spots, B) Spot-to-spot variation in the SERS intensity at $1621 \mathrm{~cm}^{-1}$ for the 3D nanoprism superlattices. All measurements were carried out at MB concentration of $10^{-4} \mathrm{M}$. .86

Figure 4.7: Bright field microscopy images and corresponding SERS spectra $\left(10^{-4} \mathrm{M}\right.$ MB) of 10 different spots

Figure 4.8: PL spectra of Alexa Fluor Phalloidin 647 with (blue) and without 3D nanoprisms superlattices (orange) on a glass substrate at laser wavelength of 633 $\mathrm{nm}$.

Figure 5.1: Fluorescent microscopy image of dispersed PS beads after DNA functionalization.

Figure 5.2: Representative SEM image showing very little binding between uncoated PS beads and uncoated gold nanoprisms. .96

Figure 5.3: A) DLS size characterization of salt effect in DNA induced binding between nanoprisms and PS microbeads, B) \& C) Representative SEM image of PS beads/ nanoprisms conjugates, i.e. nanoprisms coated single or dimers of beads, and D) Representative SEM image of PS beads/nanoprisms conjugates, i.e. bead clusters.

Figure 5.5: Fluorescence quenching of PS beads at different salt concentration. .....101

Figure 5.6: A) Melting profile of PS beads/nanoprism conjugates, monitored at 895 $\mathrm{nm}$ (the SPR of nanoprisms), B) Melting profile of control experiment, i.e. mixture of uncoated nanoprisms and uncoated PS beads in a hybridization buffer, monitored at $895 \mathrm{~nm}$.

Figure 5.7: A) \&B) Representative SEM images of 3D PS beads /nanoprisms aggregates. 
Figure 5.8: A) Raman spectrum of PS beads/nanoprism substrate (green) and SERS spectra of MB adsorbed on glass substrate (blue), PS bead aggregates (Red), \& PS beads/nanoprism aggregates (black), B) SERS spectra of MB on 3D PS beads/nanoprism aggregates at different concentrations. 105

Figure 5.9: SERS intensity of $10^{-4} \mathrm{M}$ MB using 3D PS beads/nanoprism aggregates (green) and randomly dispersed nanoprisms (dark red).

Figure 5.10: Spot-to-spot variation in SERS intensity of $M B\left(10^{-7} \mathrm{M}\right)$ at $1621 \mathrm{~cm}^{-1}$ for the PS beads/nanoprism aggregates (SERS measurements were collected from 15 different spots).

Figure 6.1: UV-vis spectra of nanoprisms before (blue) and after surface modification with PEG (dark red) and DNA (yellow)..... 118

Figure 6.2: A), B) \& C) Representative SEM images of gold nanoprism dimers .....120

Figure 6.3: Representative SEM images of control experiments. A) PEG coated nanoprisms, B) face-to-face stacking of DNA-A and DNA-A" nanoprisms, C) cluster formation due to mixed PEG/DNA surface functionalization.

Figure 6.4: A), B) \&C) Representative SEM images of assembled nanoprism trimers.

Figure 6.5: A) UV-Vis spectra of assembled gold nanoprism dimer, B) UV-Vis spectra of assembled gold nanoprism trimer.

Figure 6.6: A), B) \& C) Representative SEM images of a single nanoprism, a nanoprism dimer and a nanoprisms trimer respectively; D) \& G) Hyperspectral image of nanoprisms on a marked glass slide and the scattering spectrum of the particle marked with a red rectangle, respectively; E) \& H) Hyperspectral image of a nanoprism dimer on a glass slide and the scattering spectrum of the particle marked with red rectangle, respectively ; F) \& I) Hyperspectral image of a nanoprism trimer on a glass slide and the scattering spectrum of the particle marked with red rectangle,

Figure 6.7: A) Scattering spectra of 6 single nanoprism dimers \& B) Scattering spectra of 6 single nanoprism trimers. 
Figure 7.1: A) \& B) Dark field microscopy images of cellular internalization of DNA coated gold nanoprisms.

Figure 7.2: Representative SEM images of A) \& B) monolayers of nanoprisms on APTES functionalized glass wools and C) glass wool after the removal of nanoprisms.

Figure 7.3: Representative SEM images of A) glass beads \& B) monolayer of nanoprisms on APTES functionalized glass beads and C) a glass bead surface after removal of nanoprisms.

Figure 7.4: Time window for face-to-face assembly of nanoprisms.

Figure A1: Picture of a typical reaction vessel made up of cellulose acetate with MWCO 12KD for the synthesis gold nanoprisms.

Figure A2: Representative SEM images of A) as synthesized gold nanoprism crude product, B) purified gold nanoprisms and an image of C) as synthesized gold nanoprism crude product (Brown), and D) purified gold nanoprisms (Green). .164

Figure A3: UV-Vis absorbance spectra of purified gold nanoprisms of different sizes.

Figure A4: Representative SEM image of gold nanoprisms sample on a glass slide.

Figure A5: A) Representative SEM image of gold nanoprisms face-to-face arrangement after heating, which retained its structure after drop casting on the TEM grid, B) Representative SEM image showing the lack of face-to-face assembly of gold nanoprisms without heating.

Figure A6: Representative SEM image of a monolayer of nanoprisms on the APTES functionalized glass surface.

Figure A7: Representative STEM image of the conjugation of DNA-A" coated nanospheres with PEG | DNA-A Janus nanoprisms.

Figure A8: Additional STEM images of the conjugation of DNA-A" coated nanospheres with PEG | DNA-A Janus nanoprisms.

Figure B1: Additional SEM images of 1D face-to-face arrangement of gold nanoprisms. 
Figure B2: Additional SEM images of 1D face-to-face arrangement of gold nanoprisms.

Figure B3: Additional SEM image of 3D hierarchical organization of gold nanoprisms at A) low magnification, and B) high magnification.

Figure B4: Additional SEM image of 3D hierarchical organization of gold nanoprisms at A) low magnification, and B) high magnification.

Figure B5: Additional SEM image of 3D hierarchical organization of gold nanoprisms at A) low magnification, and B) high magnification.

Figure B6: Additional SEM images of 3D hierarchical organization of gold nanoprisms on a glass substrate.

Figure B7: Additional darkfield microscopy image of face-to-face stacking of nanoprisms at room temperature.

Figure B8: Additional darkfield microscopy image of face-to-face stacking of nanoprisms at room temperature.

Figure B9: Additional darkfield microscopy image of long-range DNA mediated ordering of nanoprisms at $65^{\circ} \mathrm{C}$.

Figure B10: Additional darkfield microscopy image of long-range DNA mediated ordering of nanoprisms at $65^{\circ} \mathrm{C}$.

Figure B11: Additional darkfield microscopy image of long-range DNA mediated ordering of nanoprisms at $66^{\circ} \mathrm{C}$.

Figure B12: Additional darkfield microscopy image of long-range DNA mediated ordering of nanoprisms at $66^{\circ} \mathrm{C}$.

Figure B13: Additional darkfield microscopy image of long-range DNA mediated ordering of nanoprisms at just below the melting temperature of 1D nanoprisms $\operatorname{stack}\left(\mathrm{T}_{\mathrm{m}} \sim 68.5^{\circ} \mathrm{C}\right)$

Figure B14: Additional darkfield microscopy image of long-range DNA mediated ordering of nanoprisms at just below the melting temperature of 1D nanoprism stacks $\left(\mathrm{T}_{\mathrm{m}} \sim 68.5^{\circ} \mathrm{C}\right)$.

Figure B15: Additional SEM images of PS bead/nanoprism conjugates: A) \& B) Nanoprism loaded single bead. 
Figure B16: Additional SEM image of PS bead/nanoprism conjugates: A) \& B) PS bead/nanoprism cluster.

Figure B17: Additional SEM images of 3D PS bead/nanoprism aggregates. ...........188

Figure B18: Additional SEM image of gold nanoprism dimers............................189

Figure B19: Additional SEM image of gold nanoprism dimers.............................190

Figure B20: Additional SEM image of gold nanoprism trimers. ...........................191

Figure B21: Additional SEM image of gold nanoprism trimers. ............................192

Figure C1: Additional Raman spectra of $10^{-4}$ M MB on pristine glass slides...........194

Figure C2: Additional SERS spectra of $10^{-6} \mathrm{M} \mathrm{MB}$ on 3D nanoprism superlattices.

Figure C3: Additional SERS spectra of $10^{-7} \mathrm{M} \mathrm{MB}$ on 3D nanoprism superlattices.

Figure C4: Additional SERS spectra of $10^{-8} \mathrm{M}$ MB on 3D nanoprism superlattices.

Figure C5: Additional SERS spectra of $10^{-9} \mathrm{M} \mathrm{MB}$ on 3D nanoprism superlattices.

Figure C6: Additional SERS spectra of $10^{-10} \mathrm{M} \mathrm{MB}$ on 3D nanoprism superlattices.

Figure C7: SERS intensity of $10^{-4} \mathrm{M} \mathrm{MB}$ at $1621 \mathrm{~cm}^{-1}$ from 5 different spots of 3D nanoprism superlattices (sample 1).

Figure C8: SERS intensity of $10^{-4} \mathrm{M} \mathrm{MB}$ at $1621 \mathrm{~cm}^{-1}$ from 5 different spots of 3D nanoprism superlattices (sample 2).

Figure C9: SERS intensity of $10^{-4} \mathrm{M} \mathrm{MB}$ at $1621 \mathrm{~cm}^{-1}$ from 5 different spots of 3D nanoprism superlattices (sample 3).

Figure C10: SERS intensity of $10^{-4} \mathrm{M} \mathrm{MB}$ at $1621 \mathrm{~cm}^{-1}$ from 5 different spots of 3D nanoprism superlattices (sample 4).

Figure C11: SERS intensity of $10^{-4} \mathrm{M} \mathrm{MB}$ at $1621 \mathrm{~cm}^{-1}$ from 5 different spots of 3D nanoprism superlattices (sample 5).

Figure C12: Additional SERS spectra of $10^{-4} \mathrm{M} \mathrm{MB}$ on bare PS beads .206 
Figure C13: Additional SERS spectra of $10^{-6} \mathrm{M} \mathrm{MB}$ on 3D PS bead/nanoprism aggregates.

Figure C14: Additional SERS spectra of $10^{-7} \mathrm{M} \mathrm{MB}$ on 3D PS bead /nanoprismsaggregates.

Figure C15: Additional SERS spectra of $10^{-8} \mathrm{M} \mathrm{MB}$ on 3D PS bead/nanoprism aggregates.

Figure C16: Additional SERS spectra of $10^{-9} \mathrm{M} \mathrm{MB}$ on 3D PS bead/nanoprism aggregates.

Figure C17: Additional SERS spectra of $10^{-10} \mathrm{M}$ MB on 3D PS bead /nanoprism aggregates.

Figure C18: Additional PL spectra of Alexa Fluor Phalloidin 647 using 3D nanoprism superlattices.......

Figure C19: Additional PL spectra of Alexa Fluor Phalloidin 647 on pristine glass slides (blank). 


\section{LIST OF TABLES}

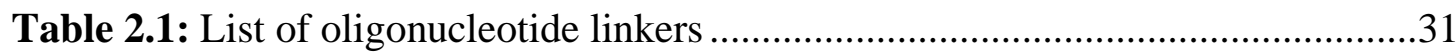

Table 2.2: Durations of nanoprisms deposition on APTES surface based on their

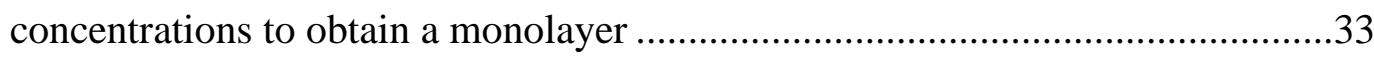

Table 3.1: Surface Zeta potential of nanoprisms before and after functionalization...61

Table 5.1: Calculation of the number of MB molecules excited for Raman .............110

Table 5.2: Calculation of the number of MB molecules excited for SERS ..............110

Table 7.1: Comparison of surface areas of different glass substrates .....................137

Table C1: Statistical reproducibility analysis of SERS performance of 3D nanoprisms superlattice 


\section{LIST OF SCHEMES}

Scheme 2.1: Schematics of face-to-face lamellar arrangement of gold nanoprisms

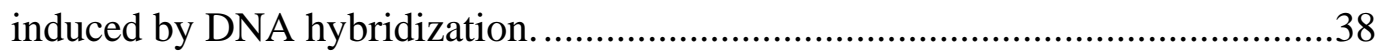

Scheme 2.2: Schematics of 1D gold nanoprism stacks during melting analysis. ........39

Scheme 2.3: Schematics of covalent coupling between carboxylate modified PS beads and amine functionalized ligands through carbodiimide chemistry......................41

Scheme 2.4: Schematics of gold nanoprism dimer and trimer formation ....................47

Scheme 3.1: Schematics for the synthesis of Janus gold nanoprisms..........................57

Scheme 5.1: Schematic representation of DNA induced loading of gold nanoprisms onto PS beads. 


\section{CHAPTER 1}

\section{INTRODUCTION}




\subsection{Motivation}

With the increasing need to derive low dimensional functional nanomaterials, the concepts of rational design and creation of novel and complex hierarchical nanostructures are continually attracting intense interest. ${ }^{1}$ Nanoparticles or nanocrystals are one of the most promising and attractive building blocks for architecting complex nanostructures due to their unique size- and shape-dependent functional properties and potential applications in various fields such as biomedicine, optics, catalysis, and electronics. ${ }^{2-3}$ The full exploitation of such unique capabilities of nanoscale objects lies in the spatial organization or assembly of these building blocks with a high degree of direction and control. ${ }^{4-5}$ Directed assembly is a self-assembly approach that provides an efficient strategy of organizing nanoscale and microscale building blocks into crystalline, patterned or other higher order functional materials with a greater control in orientation and placement. ${ }^{6-7}$ Many directed-assembly techniques including the use of pressure gradients, magnetic fields, electric fields, electron beams, lights and lasers, liquid-liquid interfaces, and template-guided assembly have been introduced to generate ordered nanoparticle superstructures. ${ }^{8-13}$ However, all of these strategies have limits in the control of particle placement and interparticle distance, and very often result in nanoparticle superlattice without welldefined, predictable and programmable crystal lattices. In contrast, DNA-mediated assembly represents a significant advance in the bottom up engineering of programmable atom equivalent nanoparticle superlattices due to the exceptional molecular recognition, tunability, and predictability of DNA mediated interactions, 
which enable a high degree of control over particle assembly to generate various 1D, 2D , and 3D nanostructures with well-defined crystal symmetry and tunable interparticle distance. ${ }^{14-15}$

So far, a diverse range of structural and functional building blocks have been reported to construct various functional metamaterials through directed assembly. Some of the most commonly used building blocks are small organic molecules, peptides, macrocycles, polymers, and metallic nanoparticles. ${ }^{6,16-20}$ However, there is still a lack of versatile building blocks that are bifunctional in nature or that take advantage of shape anisotropy and undergo asymmetric self-assembly or directed selfassembly.

Furthermore, the surface modification of nanoscale objects may not only modify the surface properties, but also yield new properties in nanoparticles for various applications. ${ }^{21-22}$ Asymmetrically functionalized Janus particles from metal nanoparticle cores containing two or more different chemical functionalities are of increasingly significant interest, because of their wide range of potential applications as surfactants, emulsifiers, water-repellent coatings, building blocks for directed selfassembly, biphasic catalysts, drug delivery vehicles, biomedical imaging and sensing contrast agents, and high-throughput immunoassay components. ${ }^{21,23}$

Despite the significant progress in the design and synthesis of Janus particles, the majority of synthesis approaches are based on the surface modification of spherical core nanoparticles. There is still an urgent need for the development of more effective synthetic protocols and creation of more complicated Janus nanostructures from 
anisotropic core particles. The objective of this dissertation is to develop a facile method for controllable asymmetric surface encoding of anisotropic gold nanoprisms for Janus particle synthesis and to demonstrate the utility of the asymmetric functionalization strategy to generate bifunctional building blocks for regioselective synthesis of nanoprism dimers and trimers through DNA-induced directed assembly. Additionally, another major goal of this study is to investigate how the shape of anisotropic nanoparticles directs and size variation affects DNA-induced 3D hierarchical self-assembly of nanoparticles as well as to understand DNA induced selfassembly and interaction between anisotropic nanoparticles and polymer beads. Finally, the study also aims to understand the plasmonic properties of targeted nanostructures such as dimer and trimer of nanoprisms and asses the applicability of 3D nanostructure of anisotropic nanoprisms and polymer beads as surface-enhanced Raman scattering and surface-enhanced fluorescence substrate.

\subsection{Gold nanoprisms}

Gold triangular nanoprisms, also known as gold nanoplates, have recently drawn significant interest due to their photo-thermal ability in the near infrared (nIR) region, ease of surface modification and unique architecture dependent properties. ${ }^{24-28}$ The triangular prisms, which are a geometrically sophisticated 2D nanostructures, have a high aspect ratio and drastically different local curvatures, i.e. flat surfaces and sharp tips. The multivalent structural features of nanoprisms, which are beyond those of symmetric nanoparticles (nano-spheres), make them promising building blocks for 
engineering novel materials and complex nano-architectures with hitherto undiscovered collective phenomena. ${ }^{29-31}$

Nanoprisms typically have a triangular prismatic shape with congruent edge lengths (1) in the range of $\sim 40 \mathrm{~nm}-1 \mu \mathrm{m}$, and a thickness of $\sim 5-40 \mathrm{~nm}$ (Figure 1.1). ${ }^{32}$ Gold nanoprisms contain sharp edges and pointed or rounded vertices, which confine electromagnetic fields on the particle and contribute to the nanoprism electronic and optical properties. ${ }^{33}$ The strong enhancement in the vicinity of the nanoprisms sharp edges make the localized surface plasmon resonance (LSPR) band tunable throughout the visible and near-IR (NIR) regions of the spectrum based on aspect ratio, symmetry

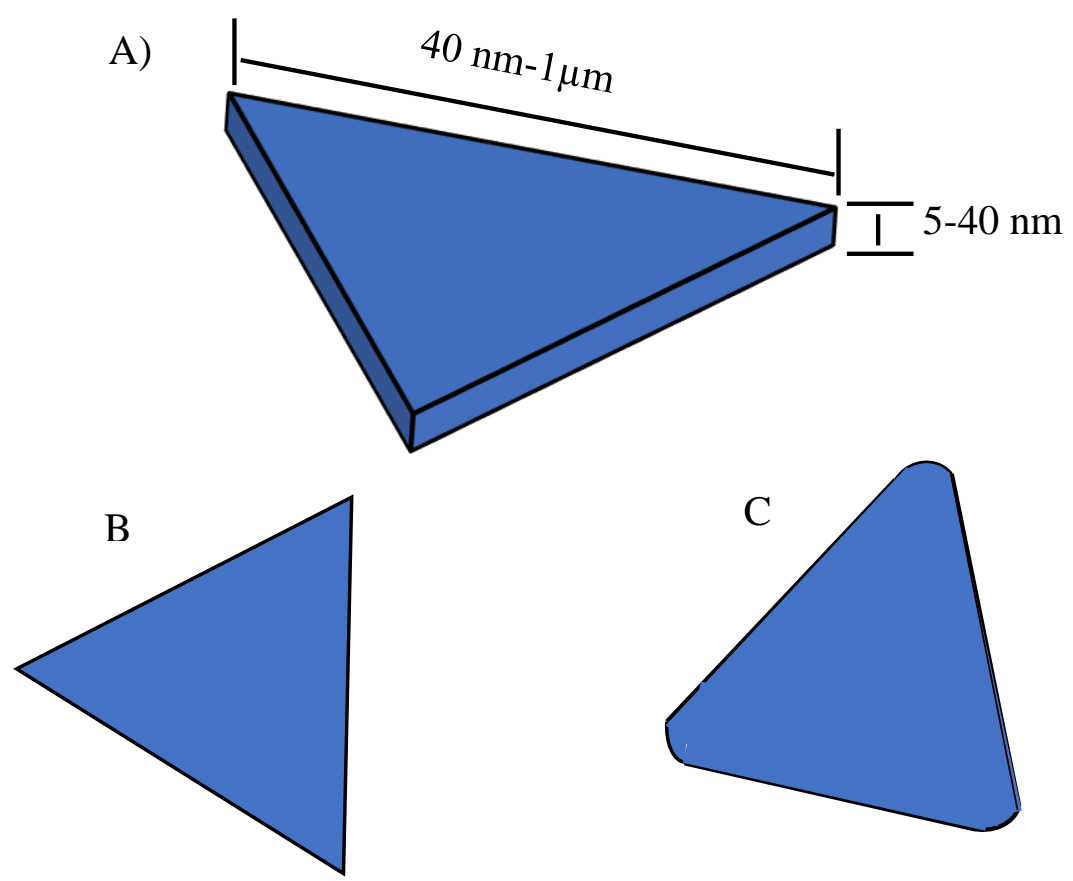

Figure 1.1: A) Dimensions of gold nanoprisms, B) Nanoprisms with sharp vertices, C) Nanoprisms with snipped vertices. 
and tip morphology. ${ }^{32,34}$ For instance, the LSPR band becomes blue shifted for nanoprisms with rounded tips while larger edge lengths red-shift the LSPR. ${ }^{35}$ Architecture dependent plasmonic properties of nanoprisms can find applications in plasmonic sensing-based molecular diagnostics, surface-enhanced spectroscopies (surface enhanced raman scattering, surface enhance fluorescence, surface enhanced infrared absorption), the synthesis of metamaterials, and photonics. ${ }^{36-37}$

Additionally, nanoprisms also have two extended, atomically flat major facets. As a result, they are essentially two-dimensional bifunctional nanoscale objects, which are quite unique among anisotropic structures synthetically available today and can be manipulated for various molecular and supramolecular assemblies due to their 1) flat surfaces with abundant surface area, which allows numerous ligand interactions and provides enhanced binding strength, 2) the ability to accommodate effective local concentrations of coating ligands due to slow ligand exchange on flat surfaces, 3) preferential shape-directed face-to-face association or lamellar arrangement of nanoprisms, which allows maximum ligand interactions. ${ }^{24,29,38-41}$

nIR-absorbing gold nanoprisms, in particular, are a highly sought-after synthetic target for their application in biomedical imaging, diagnosis, and photo thermal cancer therapy. A variety of synthetic methodologies including seeded growth methods, thermal reduction approaches, biological methods, electrochemical, and photochemical approaches and quite recently plasmon mediated synthesis have been developed to synthesize gold nanoprisms, since the first report by Milligan and Morriss in 1964. ${ }^{32,42-49}$ Most of the synthetic approaches require the use of toxic 
surfactants such as Cetyl Trimethyl Ammonium Bromide (CTAB) or

Polyvinylpyrrolidone and rely on multi-step seeding approaches. Moreover, in most cases, a lack of a clear understanding of the growth mechanism, low morphological yield (lower than 70\%) as well as the difficulty in synthesizing smaller nanoprisms have hindered the development of nanoprisms for practical applications. ${ }^{43}$ One of the promising synthetic approaches to generate nanoprisms with controlled size and high yield is the Diasynth method, which employs a regenerated cellulose membrane (RCM) with a specific molecular weight cut off (MWCO), e.g. $15 \mathrm{kDA}$, as a reaction vessel, and proceeds through reducing chloroauric acid with sodium thiosulfate. ${ }^{50-52}$ By varying the concentration of chloroauric acid and sodium thiosulfate, nanoprisms with sizes ranging from $60-200 \mathrm{~nm}$ can be synthesized. One of the drawbacks is the presence of byproduct colloidal nanoparticles (diameters $=2-10 \mathrm{~nm}$ ) and pseudospheroid particles, with diameters near $30 \mathrm{~nm}$ produced by this method. We found that two successive centrifugal washes at $180-200 \mathrm{~g}$ for 30 mins can remove over $99 \%$ of colloidal contaminants, thereby significantly increasing the purity of the nanoprisms.

\subsection{Janus Nanoparticles}

Janus particles are colloidal building blocks consisting of at least two surface regions that exhibit different chemistry, functionality and/or directionality derived from the anisotropy or asymmetry within a single set of particles. ${ }^{53-54}$ While Janus particles might possess a broad range of architectures, different chemical natures, and sizes varying in the range of a few nanometers up to tens of microns, they share a common trait of assuming an asymmetric and anisotropic structure stemming either 
from surface modifications of particles called patchy Janus particles or multiple phaseseparated regions in the core of the particle called compartment Janus particles. ${ }^{54-55}$ From a scientific perspective, the characteristics of Janus colloids fall in both microscopic and macroscopic realms. Their size is small enough to be thermally perturbed, but on the other hand, their Brownian motion is slow enough and suitable to be monitored real time with optical microscopy. ${ }^{56}$ Like in atomic and molecular systems, the predominant particle interactions governing colloidal systems include Van der Waals, electrostatic and dipolar interactions. ${ }^{36,55}$

The presence/coexistence of different attributes or asymmetric features within a single particle confers the ability of directional interactions or side selective reactivity to the Janus particles, with potential applications in diverse areas such as surfactants/emulsion stabilizers, biosensor, catalysis, display coatings, drug delivery, biomedicine, and nanomotors. ${ }^{36,53,55,57}$ Additionally, polymeric Janus particles with two chemically different and incompatible facets can interact with each other in response to changes in their environment, i.e. changes in solvent and heating conditions, and undergo hierarchical self-assembly to create complex superstructures with novel applications. ${ }^{58-59}$

Synthesis of Janus particles is carried out via either surface modification or compartmentalization. Surface modification involves asymmetric surface modification of isotropic particles while compartmentalization involves synthesizing particles with anisotropic or asymmetric shapes and/or compositions. 
Since the first report of the synthesis of Janus particles (asymmetric poly(styrene)/poly(methyl methacrylate) lattices in 1985$),{ }^{60}$ many synthetic pathways have been developed for the preparation of Janus particles including metal evaporation,${ }^{58,61}$ emulsion methods, ${ }^{62-63}$ layer-by-layer self-assembly,${ }^{64}$ biphasic electrified jetting, ${ }^{65}$ and microfluidics. ${ }^{66-67}$ Despite the disparity in various synthetic approaches, synthesis of Janus particles can be broadly classified into three categories: Self-assembly, Masking, and Phase Separation. Figure 1.2 provides a schematic representation of the three main categories for synthetic methods of Janus particles.

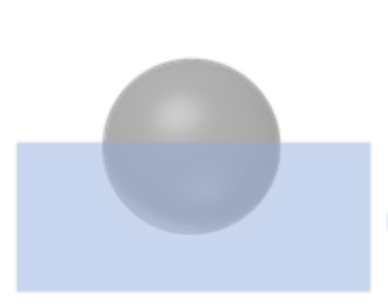

Immobilization on template

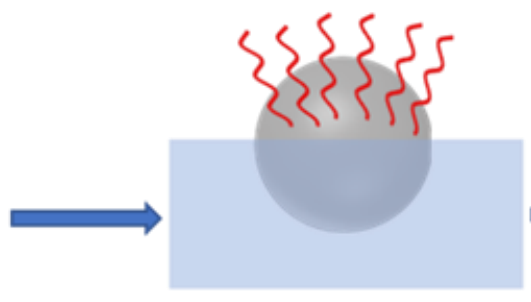

Functionalization

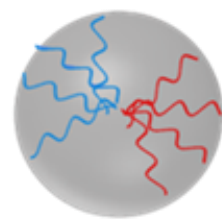

Phase separation

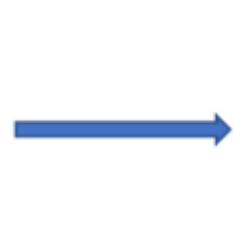

\section{Phase separation}

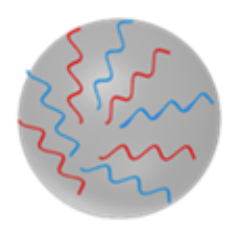

Mixing
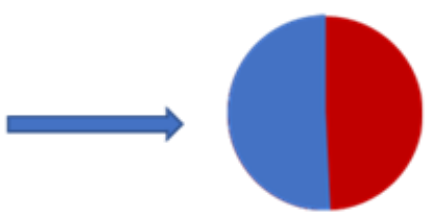

Janus particle

\section{Self-assembly}

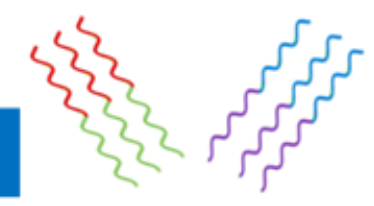

AB copolymers

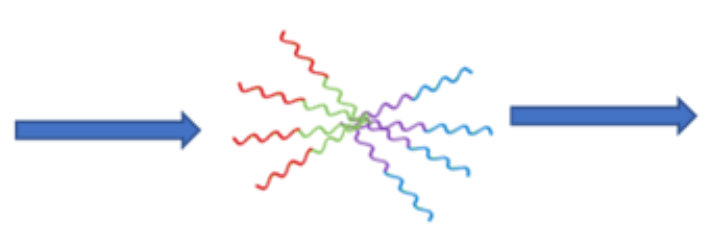

Self-assembly

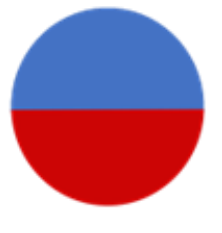

Janus particle

Figure 1.2: Three broad synthetic strategies for Janus particle synthesis. 


\subsubsection{Masking at interfaces}

The simplest way to introduce asymmetry to a symmetric object is to chemically modify one discrete portion of the object while the other portion is prevented from modification. ${ }^{53}$ This is accomplished by discretely masking one part of the object of interest and keeping it unexposed to surface coatings while leaving the other part available for surface modification. The first example of this asymmetric functionalization approach was to deposit a monolayer of isotropic particles on a solid support and then functionalize their exposed areas. ${ }^{68}$ Various methods that have been reported/devised for immobilizing particles on a solid surface including simple physisorption, ${ }^{69}$ sticky gels, ${ }^{70}$ covalent interaction, ${ }^{71}$ waxdroplet, ${ }^{72}$ and embedding in films ${ }^{73-74}$. There have also been several different strategies adopted to functionalize the particles deposited/embedded on a solid surface. Some of the functionalization processes include, but are not limited to, microcontact printing ${ }^{75}$ plasma treatment, ${ }^{76}$ reactive etching, ${ }^{77}$ sputtering,${ }^{78}$ glancing ion deposition, ${ }^{79}$ polymer chain photo-grafting, ${ }^{80}$ and attachment of metallic nanoparticles ${ }^{81}$. Although the majority of earlier reports on Janus particles created following this strategy focused on micron size particle deposition, some recent studies reported the synthesis of Janus particle with sizes down to $20 \mathrm{~nm}{ }^{82}$ Moreover, a range of non-spherical and well-defined Janus particles, for example, hybrid organic and inorganic Janus rods, have also been synthesized using a masking and de-symmetrization approach, where materials were sequentially deposited inside channels of two dimensional arrays of cylindrical pores of a thin 
film template and then the Janus particles were collected by dissolving the template into solvent. ${ }^{83-84}$ Despite a wide range of well-defined and non-spherical Janus particles having been synthesized, low yield of Janus particle produced by the methods described above have limited and hindered their use for large scale applications.

\subsubsection{Phase separation}

Another effective way to impart asymmetry into isotropic particles is utilizing the incompatibility of two immiscible substances. ${ }^{53,85}$ The principle behind this approach is to create a single set of particles with two different phases from two immiscible liquids and then create Janus particles by inducing phase separation. Incompatible surface coatings can be introduced into a single particle in various ways and hence a wide range of Janus particle consisting of inorganic, organic and polymeric substances can be readily synthesized. ${ }^{85}$ For example, Janus particles with incompatible polymer blends as well as hybrid polymeric inorganic or organic-inorganic substances have been synthesized by solvent evaporation, which

induced organic-inorganic dual phase separation within the polymer. ${ }^{86}$

Emulsion, which is an effective method to confine water insoluble polymers in a very small space, has been employed to synthesize polymeric Janus particles. The size of the droplets in the emulsion controls the dimensions of the resultant particles. The first emulsion-based Janus particle synthesis from a mixture of PS and PMMA polymer was developed by Okubo and coworkers. ${ }^{87}$ Polymer mixtures were confined within micrometer sized droplets of toluene in water and slow 
evaporation of organic solvents induced the phase separation of the two polymers, which led to separation of distinct PS and PMMA regions inside the particle. This concept of phase separation of two homopolymers was extended even further to create magnetic Janus particles. Gao and coworkers synthesized Janus particles consisting of magnetic nanoparticles in one hemisphere and poly(styrene)-blockpoly (allyl alcohol) in another ${ }^{88}$ Evaporation of the organic solvent promoted precipitation and reversible aggregation of nanoparticles from the polymer and thus separation of nanoparticles and polymer within the Janus particles. Microfluidic techniques have also been utilized for phase separated Janus particle synthesis. ${ }^{89-90}$ The principle of this technique lies either in the development of double emulsion droplets or creating a biphasic mixture of monomers, and co-extrusion and breaking of the mixture into a single emulsion droplet. ${ }^{74}$ In both cases, asymmetric droplets are allowed to solidify by inducing polymerization of monomers of droplets with UV-irradiation or thermal energy. ${ }^{91}$ Biphasic electrohydrodynamic co-jetting is another elegant and effective technique to fabricate multiphase Janus particles. ${ }^{92}$ This method is based on the application of a high electric field on two polymer solutions flowing parallel in a needle. Due to the intense electric field, their interfacial tension leads to formation of micrometer sized droplets. The rapid and instantaneous evaporation of the solvent induces solidifications of nonvolatile components and thus generates Janus particles with two compartments. 


\subsubsection{Self-assembly}

Although bottom up self-assembly of block copolymers is an elegant strategy to create a wide range of precisely defined nanostructures, the use of self-assembly has been mostly limited to formation of nanostructures which are symmetric..$^{53,57}$ Synthesis of nanostructures with broken symmetry via self-assembly of copolymers has been limited. Over the years, a number of strategies have been developed to break symmetry in self-assembly processes and generate Janus particles via selfassembly. These methods are mostly based on the co-micellization of two AB and

BC block copolymers in a solvent not favorable to the B block of the polymers. ${ }^{53,85}$ The resultant micelles contain an insoluble core made from B blocks and a separated corona of $\mathrm{A}$ and $\mathrm{C}$ blocks. The formation of single population micelles is dependent on the right balance of opposing forces and low incompatibility of two blocks. In order to avoid the incompatibility issue, mixing of two $\mathrm{AB}$ and $\mathrm{CD}$ polymers, where B and C can interact to form micelle core has been introduced. ${ }^{93}$ Another alternative to this method is to replace the mixture of diblocks by a single triblock, where the central block acts as an insoluble core. For example, Armes, et al. reported the synthesis of Janus particles along with various other micelles by dissolution of triblock poly (ethylene oxide)-blockpoly(caprolactone)-block-poly (2-aminoethy methacrylate) in water. ${ }^{94}$ Another instance of Janus particle synthesis via self-assembly of triblocks polymer was shown by Wang and coworkers, who showed that the thermodynamic incompatibility of PCL and poly(2(perfluorobutyl) ethyl methacrylate) in PEG-block-PCL-graft-poly(2- 
(perfluorobutyl) ethyl methacrylate) terpolymers give rise to asymmetric phase separation of the miceller core. ${ }^{94}$ Furthermore, self-assembly induced crystallization has been employed for the preparation of Janus micelles. Manners and coworkers synthesized non-centrosymmetric cylindrical micelles by exploiting epitaxial crystallization of poly(ferrocenylsilane)-based block copolymers in

solution. ${ }^{95}$ Additionally, various other types of asymmetric particles have been prepared by selectively crosslinking specific microdomains of bulk morphologies of triblock copolymers and dissolving the assembly in a suitable solvent. ${ }^{96-98}$ Due to remarkable progress in controlled polymerization techniques, the strategy to manipulate bulk morphologies of $\mathrm{ABC}$ triblock polymers has become a facile and versatile tool for the preparation of Janus micelles.

\subsection{DNA-mediated assembly of nanoparticles}

The utilization of DNA strands as a bottom-up self-assembly approach to construct highly programmable and predictable supramolecular constructs has seen remarkable growth over the last few decades. ${ }^{99-100}$ The first example showing the use of DNA frames as a chemical entity to construct flexible artificial DNA structures containing multiple crossover junctions between double helices dates back to the 1980s. This approach remains one of the central motifs of "Structural DNA Nanotechnology", a term that was coined and introduced by Seeman and coworkers. ${ }^{101}$ The high level of flexibility associated with multi arm junctions was not however suitable for higher order structures. In order to enhance stability, Seeman and coworkers introduced the concept of DNA double crossover (DX) molecule formed by 
joining two four -way junctions. ${ }^{102}$ These molecules, which contain sticky ends on each side for hybridization and could be assembled into higher order structures, are called DNA tiles. ${ }^{103}$ While the fabrication of DNA tiles represented an important step forward in the area of DNA-based self-assembly, a significant breakthrough was made when a conceptually different approach, known as 'DNA origami', involving programming DNA sequences to fold into intricate geometries at the nanoscale, was demonstrated by Joyce and coworkers. ${ }^{104}$ Typically, these geometries allow the precise positioning and site specific arrangement of nanoparticles by the placement of single stranded binding regions that can interact with the DNA sequences that are attached to particles. The significant capability of this technique was illustrated by Rothemund in 2006 with the fabrication of numerous planar 2D DNA structures with remarkable complexity. ${ }^{105}$ A significant advancement in DNA origami techniques was made in 2009 by Shih and coworkers, when this strategy for constructing arbitrary 2D shapes was extended to 3D DNA origami structures. ${ }^{106}$ Since its first use in 2006, DNA origami has been widely used to fabricate numerous well-defined 2D and 3D structures including twisted ribbons, bent arms with a full range of internal angles, notched gears of different sizes, and three-dimensional spherical wireframe objects. ${ }^{107-}$ 108

Another fundamentally different route for synthesizing nanoscale DNA structures is to utilize the complementary DNA-DNA interaction as chemical bonder and a nanoparticle surface as a template for the impregnation and orientation of DNA linkers. Unlike other DNA hybridized systems, where hybridization and intertwining 
of DNA strands determines stability and rigidity, in this case, it is the inorganic core of nanoparticles that provides the rigidity for directional interactions of DNA. ${ }^{109}$ Therefore, many of the design rules, such as multiple crossover junctions, associated with the "DNA tiles" strategy becomes trivial for nanoparticle-based DNA hybridization systems, where it is prerequisite that inorganic nanoparticle is functionalized with a dense coverage of DNA linkers anchored on the particle surface. In general, nanoparticle-based DNA hybridization systems use nanoparticles functionalized with DNA chains that hybridize with neighboring functionalized particles through the collective interaction of surface bound DNA strands. The size, symmetries and lattice groups of the hybridized nanoparticle superlattice are ultimately determined by the size and shape of the nanoparticle, length of DNA strands, the sequence of the complementary DNA linker, and DNA loading density. ${ }^{99}$, 106

\subsubsection{DNA-mediated assembly of spherical nanoparticles}

The utility of DNA to program nanoparticle-based structures was first demonstrated and pioneered by Mirkin and coworkers, and Alivisatos and coworkers in 1996. ${ }^{15,110}$ Mirkin's group functionalized two batches of $13 \mathrm{~nm}$ gold nanoparticles with non-complementary oligonucleotides containing thiol moieties at the 3 ' or 5 ' terminal. ${ }^{15}$ The subsequent addition of DNA duplex linkers with sticky ends that were complementary to the oligonucleotide sequence anchored to the two batches of gold nanoparticles induced self-assembly of nanoparticles into macroscopic materials. Alivisatos and coworkers adopted a slightly different 
approach. Instead of oligonucleotide functionalization of two batches of gold nanocrystals, they attached single stranded oligonucleotides to one set of gold nanocrystals. The addition of a DNA template that contains oligonucleotide sequences complementary to the sequence attached to the nanocrystals yielded dimers and trimers of nanocrystal. ${ }^{110}$

DNA sequences for use with gold nanoparticles are usually modified with thiol moieties at the 3' or 5' terminals, a spacer containing poly thymine (T) or adenine (A) and a guanine and cytosine (GC) rich sticky end section, which interacts with its complementary strands via Watson-crick base pairing. ${ }^{111}$ The thiol moieties attach the DNA strands to a gold nanoparticle surface and orients the oligonucleotide sequences in a surface-normal fashion, which allows them to bind with their complementary strands. A poly T or A spacer that doesn't have significant affinity for nanoparticle surface separates the GC rich sticky end from the nanoparticle surface to prevent steric hindrance and facilitate base pairing. $G$ and $\mathrm{C}$ bases have stronger hydrogen bonding capability than $\mathrm{A}$ and $\mathrm{T}$, therefore $\mathrm{G}$ and $\mathrm{C}$ are chosen for sticky end composition. ${ }^{108,112}$

The design of the DNA sequence plays a pivotal role in the symmetry and ordering of DNA-based spherical nanoparticle superlattices. The majority of the initial studies utilized linker oligonucleotides containing regions that are complementary to DNA strands anchored to particle surfaces. ${ }^{15,113}$ While the linker strand facilitates complementary DNA binding events generating assembled nanoparticle structures, these materials showed only short-range ordering. 
Nanoparticle structures showing long range ordering to generate larger crystals was subsequently accomplished by thermal annealing approaches which can be carried out in two ways. ${ }^{114-115}$ First, DNA functionalized nanoparticles are heated above the melting temperature of the assembled nanoparticle structure followed by slow cooling and second, DNA functionalized nanoparticles are mixed and allowed to hybridize, followed by annealing a few degrees below the meting temperature. Mirkin and coworkers showed that a binary mixture of gold nanoparticles, when crystallized by heating above $\mathrm{T}_{\mathrm{m}}$ followed by slow cooling, resulted in disordered FCC lattices whereas a non-close BCC lattice was obtained by allowing the binary mixture to hybridize at room temperature followed annealing below $\mathrm{T}_{\mathrm{m}}{ }^{113}$ Another contemporary study carried out by Gang and coworkers also illustrated the longrange ordering of these building blocks into well-defined crystals by using a thermal annealing approach. ${ }^{115}$ The implication of these studies is that the competition between entropic and enthalpic effects during the assembly process dictates the formations of different crystals at different temperatures. And, the assembly of nanoparticles into a well-order crystal that is formed via thermal annealing and allows maximum DNA hybridization events is a thermodynamically favored process, with the initially formed structure (pre-annealing) in a 'kinetically trapped' state. ${ }^{116-117}$ These findings were then subsequently corroborated by many other investigations and form the basis for the design rules of DNA-mediated nanoparticle superstructures. ${ }^{118}$ These rules were validated by a simple geometric model known as 'complementary contact model' that can be used to predict the thermodynamically favored nanoparticle superlattice as the assembly of particles 
allowing maximum complementary contacts and hence maximum DNA hybridization. ${ }^{119}$

DNA directed assembly is not limited to gold nanoparticles. As the only prerequisite for creating DNA bonding is the dense DNA functionalization of a nanoparticle with solid material core, this method of functionalization has been extended to other types of inorganic nanoparticle with a variety of compositions such as CdSe/ZnS quantum dots (QD), CdSe/CdTe/@ZnS QDs, iron oxide $\left(\mathrm{Fe}_{3} \mathrm{O}_{4}\right)$ particles, platinum $(\mathrm{Pt})$, silver $(\mathrm{Ag})$ and Palladium $(\mathrm{Pd})$ nanoparticles. ${ }^{115,120-121}$

\subsubsection{DNA-mediated assembly of anisotropic particles}

Although isotropic nanoparticles with added valency are attractive for generating nanoparticle dimers, trimers, and other regioselective structures, the realization of crystalline superstructures with low symmetry has been mostly elusive. ${ }^{99,108}$ One way to introduce directionality similar to atomic structures is to use polyhedron building blocks, which have not only defined edges and/or tips with high chemical reactivity that can be selectively functionalized, but also flat regions with larger surface areas, which tend to direct face-to-face association of these building blocks. ${ }^{99,122}$ The majority of anisotropic nanoparticle synthesis requires surfactants such as CTAB. The surfactant at the tips and edges are labile and hence these regions of curvature can be more readily functionalized with thiolated linkers, which allows selective incorporation of other nanoparticles through complementary

DNA interactions or biomolecules at those regions. ${ }^{123}$ This has been explored to 
generate DNA hybridized nanostructures, where spheres were selectively attached to tips and/or edges of nanoprisms and nanorods. ${ }^{124-125}$

Packing of anisotropic building blocks without the assistance of ligands has been carried out via depletion attraction. ${ }^{126} \mathrm{~A}$ binary mixture of particle shapes might result in shape segregation driven by entropy and maximum packing considerations. ${ }^{127}$ However, in case of nanoparticle-supported DNA hybridized systems, the role of nanoparticles in DNA mediated assembly becomes irrelevant to the role that DNA sticky ends play. Therefore, conceptually, the engineering and design of DNA sequences and functionalization to realize directional binding interactions of DNA anchored on anisotropic particles virtually remains the same as with isotropic particles. The first example of DNA guided crystallization of anisotropic nanoparticles such as rods, triangular prisms, rhombic dodecahedra, and octahedra was demonstrated by Mirkin and coworkers. ${ }^{29}$ They showed that particle geometry played a pivotal role in the symmetry and dimension of the crystal structure formed from each anisotropic building block. As the particle's flat surfaces contain more DNA density, the most preferential interaction would be face-to-face association of flat surfaces maximizing hybridization interactions. This results in the formation of 2D hexagonal close-packed lattices for rods, 1D lamellar arrangement for nanoprisms, face centered cubic (FCC) lattice for rhombic dodecahedra and body centered cubic (BCC) lattice for octahedra. ${ }^{29}$ Additionally, DNA-induced crystallization of binary mixtures of two different anisotropic particles has been studied. ${ }^{128-129} \mathrm{Lu}$, et. al. have demonstrated that a binary mixture 
of spheres and cubes yielded an FCC lattice while a mixture of spheres and octahedrons produced a $\mathrm{CsCl}$ type lattice structure. ${ }^{129} \mathrm{O}$ 'Brien, et. al. carried out a detailed study on the effect of size complementarity and shape complementarity on co-crystallization. ${ }^{128}$ It was found that co-crystallization of cubes of two different lengths constantly gave rise to the formation a $\mathrm{NaCl}$ type lattices but with a reduced crystallinity as the length of cube was decreased. They also studied shape complementarity of nanoparticles by co-crystallizing cubes of same size with different facet types, i.e. different convexity and concavity, which yielded an FCC lattice with a coordination number 6 . The implication of these studies is that the underlying anisotropy of anisotropic nanoparticles determines the crystal lattice formed and nanoparticle shape and size complementarity and can be utilized to generate new materials via co-crystallization of anisotropic nanoparticles while controlling the structural properties of those materials. ${ }^{24,30,99}$

\subsection{Metal nanoparticle coated polymer beads}

In recent years, the application of micron sized spherical polymer beads as a carrier medium or support for metal nanoparticles, or as a geometric restriction template for the surface manipulation of metal nanoparticles has drawn significant attention. ${ }^{130}$ Polymer beads not only protect metal nanoparticles from the environment and prevent aggregation and leaching of nanoparticles, but also facilitate mass and heat transfer. ${ }^{131}$ The utilization of nanoparticle-polymer bead hybrid nanostructures depends on the control of the deposition and distribution of nanoparticle on the beads' surface. ${ }^{131-132}$ 
A plethora of studies have focused on the fabrication of nanoparticle-polymer bead composites. These studies can be categorized into either in-situ techniques, where nanoparticles are grown within a polymer matrix or ex-situ techniques, where nanoparticles are impregnated onto polymer beads via surface modification of the beads. In-situ reduction techniques comprise binding of metal precursors on unmodified or modified polymer beads and subsequent in-situ reduction to produce the desired metal nanoparticles on the beads' surface. ${ }^{133}$ Quite recently, Jeong and coworkers reported a facile method to fabricate metal nanoparticle (silver, iron oxide and Titanium oxide) coated polystyrene (PS) beads by a catechol conjugated polymer. ${ }^{132}$ But these strategies are associated with irregular coverage and low density of particles. In order to circumvent this low and irregular coverage of particles, some other methodologies including metal ion presoaking, and metal seeding have been reported. ${ }^{134-135}$ Yet, it is very challenging to prevent aggregation and control monodispersity of nanoparticles through these processes.

On the other hand, a number of ex-situ nanoparticle loading techniques have also been reported. ${ }^{136-139}$ One of the advantages of ex-situ techniques is that metal nanoparticles with the desired shape, size and mono-dispersity are preformed and subsequently loaded onto the polymer beads. The first example of ex-situ techniques was the electrostatic deposition of preformed anionic colloids on a polycationic polystyrene surface. ${ }^{136}$ Two other studies that utilized electrostatic interactions to construct polymer-nanoparticle conjugates were layer-by-layer assembly ${ }^{137,139-140}$ and nanoparticle impregnation into beads functionalized with polyelectrolytes. ${ }^{138}$ Although 
these methods, especially layer-by-layer assembly, were able to accomplish uniform and controlled nanoparticle coating on the polymer beads, the assembly may not be stable in solutions with high ionic strength. To overcome these limitations and drawbacks, Lee and coworkers reported a solvent controlled swelling and hetero coagulation method to synthesize highly light scattering metal nanoparticle coated polystyrene (PS) beads. ${ }^{130-131}$ Their approach was based on the simple addition and removal of the solvent, tetrahydrofuran (THF), which induces swelling of PS beads and hetero-aggregation of PVP-stabilized nanoparticles and PS beads. ${ }^{130-131}$ Quite recently, biomolecules such as DNA have also been utilized to fabricate nanoparticlePS beads conjugates. Wu and coworkers reported a DNA-mediated assembly strategy for dense immobilization of gold nanoparticles onto PS beads. ${ }^{141}$ They also added Ag shells on gold nanoparticles immobilized on PS beads to maximize the number of nanogaps for plasmonic field/ SERS enhancement. ${ }^{141}$

Metal nanoparticle-polymer composites have been widely used in various applications. The majority of the studies focused on exploring nanoparticle (Au and Ag) - polymer bead conjugates as surface enhanced Raman scattering (SERS) substrates. The interparticle distance of plasmonic nanoparticles in their assembly are plasmonic hot spots, which leads to significant Raman enhancement. ${ }^{130-131,141-143}$

Because of their large surface area, polymer microbeads act as a template for a large ensemble of plasmonic hot spots on their outer surface. Additionally, because of the highly average plasmonic response, the enhancement in Raman scattering from bead to bead is almost identical, which allows SERS measurements at a very low bead 
concentration. ${ }^{143}$ The larger size or surface area allows immobilization of thousands of nanoparticles on the bead surface, which acts as a geometric restriction template and facilitates asymmetrical functionalization of nanoparticles. ${ }^{144-145}$ Mirkin and coworkers employed magnetic polystyrene microparticles with iron oxide cores as a support for impregnating gold nanoparticles using DNA mediated interactions, where the particles could be separated by heating. ${ }^{146}$ The section of the particles attached to microparticles was afforded different lengths of DNA linker through introducing a 15mer DNA extension linker that is complementary to half of the 30-mer DNA sequence on the rest of the particle surface. Then, gold nanoparticles that are functionalized with DNA sequences that can bind to the other half of 30-mer DNA sequence were exposed to the microparticles. In the final step, a $\mathrm{T}_{4} \mathrm{DNA}$ ligase was used to catalyze the formation of a phosphodiester bond between the $3^{\prime}$-hydroxyl and the $5^{\prime}$-phosphate of the extension linker and the oligonucleotide attached to the gold nanoparticles. Additionally, nanoparticle-polymer bead conjugates have also been widely explored for catalytic applications. ${ }^{133,}$ 147-148 The catalytic efficiency of metal nanoparticles is mostly hindered by their small size and the possibility of particle aggregation. To this end, polymer beads not only prevent nanoparticle aggregation, but also release mechanisms can be used so the particles can be isolated from the beads and recycled. Moreover, owing to the high dispersion of polymer beads in the reaction medium, catalytically active sites of metal nanoparticle surfaces are more accessible to reactant, which can accelerate the chemical reaction. Metal nanoparticle-coated polymer beads have been used for numerous other applications, including immunoassays, ${ }^{149}$ flow cytometry, ${ }^{150}$ photonics, ${ }^{151}$ medical imaging, ${ }^{152}$ and drug delivery. ${ }^{153}$ 


\subsection{Optical properties of DNA guided gold nanostructures}

Structural DNA nanotechnology emerges as a robust pathway for the directed self-assembly and precise control of the placement of metal nanoparticles to generate plasmonic nanostructures for various applications, including surface enhanced Raman scattering (SERS), surface enhanced Fluorescence (SEF) and molecular sensing based on plasmonic coupling of metal nanoparticles. For instance, Wang and coworkers reported a DNA based fabrication strategy to generate symmetric and asymmetric gold nanoparticle dimers and investigated the effect of nanoparticle size and interparticle distance on the plasmonic coupling between nanoparticles in dimer structures. ${ }^{154}$ They found that the surface plasmon resonance of nanoparticles increasingly red shifted with increasing particle size and decreasing interparticle distance. They further extended the use of DNA scaffolds for heterogeneous and modular assembly of gold nanorods and spherical nanoparticles and demonstrated that only plasmonic coupling between nanorods induces plasmonic shift while no plasmonic shift was observed when spherical nanoparticles were placed between nanorods. ${ }^{155}$ Yan and coworker demonstrated the unique plasmonic responses of gold nanorod dimers at various predetermined inter-nanorod angles, which were accomplished by precisely orienting the nanorods on a triangular DNA scaffold. ${ }^{156}$

Plasmonic structures can create highly enhanced local electromagnetic fields that can interact with a fluorophore, possibly leading to fluorescence enhancement, depending on the excitation wavelength as well as the radiative and nonradiative

decay rates of the fluorescent dye. ${ }^{157}$ Programmability of DNA origami allows the 
precise placement of fluorophores next to metal nanoparticles or within the interparticle spacing of nanoparticle dimers known as plasmonic nanoantenna, which can significantly enhance the fluorescence signal of the single-molecule emission. Acuna and co-works introduced a DNA induced fabrication of a nanoantenna by attaching single nanoparticles or nanoparticle dimers to a DNA origami structure that also contained a docking site for attaching the fluorophore near the plasmonic hot between nanoparticles. ${ }^{158} \mathrm{~A}$ maximum of 117 -fold fluorescence enhancement was achieved for a dye molecule positioned in the 23-nanometer spacing between 100nanometer gold nanoparticle dimer due to the highly enhanced local field created by the plasmonic nanoantennae. They subsequently reported a new generation of DNA origami structures for the fabrication of a dimer nanoantennae with a reduced interparticle spacing, which gave rise to a maximal fluorescence enhancement of 5468 and single-molecule detection at a $25-\mu \mathrm{M}$ background fluorophore concentration. ${ }^{159}$ Quite recently, Mirkin and coworkers combined top down lithography and bottom up colloidal crystal engineering with DNA to contract a 2D plasmonic nanoantennae consisting of an array gold nano-cubes with the molecular level control over the placement of fluorescent dye using DNA. ${ }^{160}$ They demonstrated the solvent responsive fluorescence emission of two dyes, which exhibited different fluorescence behavior based on the lattice mode resonance that is tunable through lattice spacing, and gap mode resonance that was tunable through DNA length and choice of solvent.

Structural DNA nanotechnology not only enables the assembly and organization of nanoparticles with tunable spacing, but also allows the placement of Raman probes 
in the nanogaps or hotspots which enhances the EM field and plasmonic coupling, leading to enhancement in SERS signal. Lan and coworkers reported a DNA guided self-assembly approach to construct gold nanoparticle dimers and systematically investigated the effect particle size and interparticle distance tunable with the change in DNA length on the ensemble SERS properties of dimers. ${ }^{154}$ They found that increasing nanoparticle size from $13 \mathrm{~nm}$ to $20 \mathrm{~nm}$ and $40 \mathrm{~nm}$ resulted in the gradual increase of the SERS intensity while increasing the interparticle distance from 5 to 10 and $15 \mathrm{~nm}$ led to dramatic decrease in the SERS intensities. Zheng and coworkers presented a DNA guided hierarchical self-assembly of core satellite plasmonic nanostructures of gold nanoparticles for a highly sensitive near IR SERS based sensor. ${ }^{161}$ They demonstrated that plasmonic core satellite structures not only cost a fraction of the cost of lithographic techniques, but also show superior SERS performance compared to commercial SERS substrates in both SERS enhancement and repressibility. The use of DNA origami also offers the remarkable capability to assemble nanoparticles into complex plasmonic SERS substrates with a greater control in tuning interparticle spacing and the placement of problem molecules in the hot spots. ${ }^{162}$ While the majority of DNA origami based SERS substates are based on assembly of a small number of nanoparticles, Zhoe and coworkers employed a DNA hexagon to expand the structural complexity of SERS metamolecules by organizing more nanoparticles into sophisticated configurations. ${ }^{163}$ They were able to assemble $30+$ nanoparticles into hexagon clusters and connect the hexagon clusters to form dimers, trimers and 1D chains of hexagon clusters of nanoparticles and showed that 
1D chain metamolecules showed stronger interparticle electromagnetic fields and hence stronger SERS response that hexagon monomers and dimer.

\subsection{Organization of the thesis}

Chapter 1 provides motivation for this study and background on various synthetic approaches and applications of nanoprisms and Janus particles, DNA mediated assembly strategies and synthetic approaches for impregnating metal nanoparticles on polymer beads. Chapter 2 provides combined experimental descriptions for the research findings presented in Chapters 3-6. Chapter 3 discusses a facile method for facet selective asymmetric functionalization of gold nanoprisms for Janus particle synthesis. Chapter 4 illustrates DNA-mediated 3D hierarchical organization of gold nanoprisms into 3D superlattices and their application for SERS and SEF. Chapter 5 describes DNA mediated assembly of anisotropic nanoprisms, and carboxylate modified polystyrene beads into 3D SERS substrates. Chapter 6 provides the regioselective synthesis of gold nanoprism dimers, and trimers for plasmonic applications. Chapter 7 summarizes the research findings of this study and put forwards suggestions for future directions. 


\section{CHAPTER 2}

\section{EXPERIMENTAL DETAILS}




\subsection{Materials}

Hydrogen tetrachloroaurate (III) hydrate $\left(\mathrm{HAuCl}_{4} \cdot 3 \mathrm{H}_{2} \mathrm{O}\right)$, methylene blue, high purity biological stain $\left(\mathrm{C}_{16} \mathrm{H}_{16} \mathrm{CIN}_{3} \mathrm{~S} . \mathrm{xH}_{2} \mathrm{O}\right)$ were purchased from Alfa Aesar (Ward Hill, MA). Sodium thiosulfate pentahydrate, phosphate buffer solution, dithiothreitol (DTT) at $1 \mathrm{M}$ concentration in $\mathrm{H}_{2} \mathrm{O}, 1$-hexadecanethiol ( $\left.\geq 95.0 \% \mathrm{GC}\right)$, carboxylatemodified polystyrene, fluorescent yellow green (aqueous suspension, $1.0 \mu \mathrm{m}$ mean particle size), (3-aminopropyl) triethoxysilane (Sigma-Aldrich $\geq 98.0 \%$ ) \& cellulose acetate dialysis tubing (43 mm wide; $12 \mathrm{kDa}$ molecular weight cutoff) were purchased from Sigma-Aldrich (St. Louis, MO). Ethanol (200 proof, 100\%) was purchased from Decon labs, Inc. Oligonucleotides sequences (thiolated) 5'-ATA-ACC-ATT-GTAAAT-TAATTA-3' (DNA-A ) and its complementary 3'-TAT-TGG-TAA-CAT-TTAATT-AAT-5'(thiolated) (DNA-A") were purchased from Integrated DNA Technologies (Coralville, IA). Microscopic slides $\left(3^{\prime \prime} \times 1^{\prime \prime} \times 1 \mathrm{~mm}\right)$ were purchased from VWR International, LLC (Rednor, PA). Illustra NAP-25 columns were purchased from General Electric Healthcare (Buckinghamshire, United Kingdom). NANOpure ultrapure water (Barnstead, resistivity 18.2 M $\Omega-\mathrm{cm}$ ) was used for all aqueous solutions unless stated otherwise. 


\subsection{List of oligonucleotide linkers used in this study}

Table 2.1: List of oligonucleotide linkers

$\begin{array}{lll}\text { DNA-A } & \begin{array}{l}\text { Adenine-rich } \\ \text { oligonucleotide }\end{array} & \begin{array}{l}\text { (thiolated) 5'-ATA-ACC-ATT-GTA-AAT-TAA- } \\ \text { TTA-3' }\end{array} \\ & & \\ \text { DNA-A' } & \text { Amine-modified thymine- } \\ & \text { rich oligonucleotide } & \\ & & \\ \text { 3'-TAT-TGG-TAA-CAT-TTA-ATT-AAT-5'-NH } 2\end{array}$

\subsection{Procedures}

\subsubsection{Chemical synthesis and purification of gold triangular nanoprisms}

Gold nanoprisms were synthesized using the Diasynth process following literature precedent. ${ }^{164-165}$ This method involves the reaction between sodium thiosulfate $\left(\mathrm{Na}_{2} \mathrm{~S}_{2} \mathrm{O}_{3}\right)$ with a gold salt, tetrachloroauric acid $\left(\mathrm{HAuCl}_{4}\right)$ in a traditional one-step or two-step process without the need of additional templates, capping reagents, or seeds. In addition, the surface plasmon resonance (SPR) of the products is tunable based on reaction parameters such as concentration and temperature. 
Briefly, $32.6 \mathrm{~mL}$ of $1.76 \mathrm{mM} \mathrm{HAuCl}_{4}$ in water was added to a $12 \mathrm{~cm}$ section of dialysis tubing followed by quickly adding $7.4 \mathrm{~mL}$ of $3 \mathrm{mM}$ aqueous sodium thiosulfate solution with agitation for 5 seconds. The membrane was then clipped and submerged in a circulated bath of DI water $(\geq 25 \mathrm{~L})$ maintained at $27^{\circ} \mathrm{C}$ by a Thermo (Waltham, MA) NESLAB RTE-221 Circulator. After reacting for 1 hour the membrane was removed and the solution was emptied into a $40 \mathrm{~mL}$ plastic tube. The poly-disperse particle solution was a mixture of nanoprisms and pseudospheroid particles, with the latter contributing almost $99 \%$ of particles. Nanoprisms were separated from pseudo-spheroid particles through 2x 30 mins long centrifugations at a speed of 180-200 g. The plasmon resonance band for pseudospheroid particles at $540 \mathrm{~nm}$ is completely diminished after centrifugal separation as the pseudo-spheroids were removed from the particle mixture and a high purity nanoprism solution was obtained.

\subsubsection{Synthesis of Janus nanoprisms}

Silica glass slides were thoroughly cleaned with an alcohol base bath and aqua regia, and then exposed to APTES for 1-2 hours. Nanoprisms was deposited on the APTES surface via drop-casting for 5-10 minutes, depending on the optical density of the nanoprism suspension so that a monolayer of nanoprisms was formed. Table 2.2 shows the concentrations of nanoprisms and corresponding duration of nanoprisms depositions that ensures the formation of monolayer of nanoprisms on the APTES surface. 
Table 2.2: Durations of nanoprisms deposition on APTES surface based on their concentrations to obtain a monolayer

\begin{tabular}{cc}
\hline $\begin{array}{c}\text { Concentrations, Optical density } \\
(\mathrm{OD})\end{array}$ & Duration of deposition (min.) \\
\hline $6-8$ OD & 12 Min \\
$8-10$ OD & 10 Min \\
$>10$ OD & 6 Min \\
\hline
\end{tabular}

The exposed nanoprism facets were coated with either hexadecane thiol (HexaD) or thiolated poly(ethylene) glycol (PEG) using the well-known affinity of sulfur for gold surfaces. Excess coating molecules were removed by sonication and rinsing with a suitable solvent. Half-coated nanoprisms were then lifted off from APTES with 4-5 hours mild sonication using potassium carbonate at $\mathrm{pH} 11$. The potassium carbonate buffer was not found to cause aggregation of nanoprisms, as shown by monitoring the nIR LSPR bandwidth of the nanoprisms after lift-off. The coating for the surface-protected facet of the nanoprisms can be applied in two ways. First, nanoprisms were exposed to the second coating agent during lift-off by adding the agent directly to the sonication buffer so that the uncoated facet was coated immediately as the nanoprisms were lifted off from the APTES surface. Alternatively, half-coated nanoprisms were purified after lift-off by centrifugal washing multiple times followed by re-suspension in nanopure water. The second coating agent, a thiolated 21-mer adenosine-rich oligonucleotide (DNA-A) was 
then applied on half-coated nanoprisms, following literature precedent. ${ }^{29} \mathrm{No}$ measurable difference was observed between the products of the two methods.

\subsubsection{Functionalization of nanoprisms with oligonucleotide}

Prior to the functionalization of Au nanoprisms, thiolated oligonucleotide was treated with 0.1 $\mathrm{M}$ dithiothreitol (DTT) in disulfide cleaving buffer (0.17 M, pH 8) to remove the thiol protecting group. Then, the oligonucleotide solution was purified using a desalting column (Nap-25, DNA grade, GE Healthcare). The purified nanoprisms were functionalized by using modifications of literature procedures. ${ }^{122,165}$ Briefly, 1.87 OD260 $(2.5 \mu \mathrm{M})$ of purified thiolated DNA was added to 1.0 OD of Au nanoprisms and allowed to react while shaking (1100 rpm, $22^{\circ} \mathrm{C}$ ) for 30 min to 1 hour using an Eppendorf Thermomixer. The particle and DNA mixture were then brought to $0.01 \%$ SDS (sodium Dodecyl sulfate) in $0.01 \mathrm{M}$ sodium phosphate buffer. Then, particle solutions were slowly treated with $\mathrm{NaCl}$ and sequentially brought to $0.05 \mathrm{M}, 0.1 \mathrm{M}, 0.2 \mathrm{M}$, and $0.3 \mathrm{M} \mathrm{NaCl}$ with $30 \mathrm{~min}$ between each addition. After the final salt aliquot addition, the particle solution was allowed to sit overnight. The next day, the particle solution was centrifuged (2000 rpm, 10 mins) 3-4 times to remove the unreacted oligonucleotide and the pellet was resuspended in $0.01 \mathrm{MPBS}$ or nanopure water.

\subsubsection{Functionalization of nanoprisms with 1-Hexadecane thiol}

A 3 OD dispersion of gold nanoprism solutions were spun down and resuspended in $1 \mathrm{~mL}$ ethanol. $30 \mathrm{mM}$ hexadecane thiol in ethanol was added to 
particle solution (10 $\mu \mathrm{M}$ of hexadecane thiol for per OD of gold nanoprisms) and the mixture was allowed to react for 3-4 hours. In order to remove unreacted hexadecane thiol, the particle mixture was centrifuged at $2000 \mathrm{~g}$ for $10 \mathrm{mins}$ and washed with ethanol for three times.

\subsubsection{Functionalization of nanoprisms with thiol modified polyethylene glycol (PEG-SH, 2k MW)}

$2 \mathrm{~mL}$ of purified gold nanoprisms were incubated in thiolated PEG (2k MW) solution in water $(10 \mu \mathrm{M}$ concentration of thiolated PEG for per $\mathrm{mL}$ of gold nanoprisms). The solution was shaken with a rotating shaker at room temperature for 5-6 hours. The coated nanoprisms were washed 3 times with NANOpure water by successive centrifugation (3000g, 10 mins). After the final wash, PEG-modified nanoprisms were taken to $0.01 \mathrm{M}$ phosphate buffer $(\mathrm{pH} \sim 7.4)$ and stored at $4^{\circ} \mathrm{C}$.

\subsubsection{Selective attachment of gold nanospheres to major facets of gold nanoprisms}

To selectively attach DNA-A" coated gold nanospheres to DNA-A -containing facets of Janus PEG | DNA-A nanoprisms, a $200 \mu \mathrm{L} 1.2$ OD dispersion of Janus nanoprisms was mixed with $100 \mathrm{uL}$ of 0.4 OD gold nanosphere solution in a DNA hybridization buffer $(0.01 \mathrm{M}$ phosphate buffer $+0.1 \mathrm{M}$ sodium Chloride). The mixture was then heated to $90^{\circ} \mathrm{C}$ and left to cool down to room temperature for hybridization. Control experiments were carried out by mixing $200 \mu \mathrm{L}$ of both DNA-A | DNA-A nanoprisms and PEG | PEG nanoprisms with $100 \mu \mathrm{L}$ of 
nanospheres in the hybridization buffer at $90^{\circ} \mathrm{C}$ and allowed to cool down slowly to room temperature.

\subsubsection{Functionalization of glass slides with Amino groups with APTES}

Prior to functionalization, silica glass slides were thoroughly cleaned and washed in a base bath and with aqua regia. Clean glass slides were taken in $2 \%$ Aminopropyltriethoxysilane (APTES) solution in 70\% ethanol and allowed to react for 1-2 hours. Then glass slides were sonicated for 10 mins with $70 \%$ ethanol, and water for another $10 \mathrm{~min}$.

\subsubsection{Functionalization of glass slides with Poly(siloxanes)}

$1 \mathrm{~mL}$ of RainX was applied on clean and dried glass slides with a piece of dry cloth or paper towel. RainX was then allowed dry for 10-20 mins or until a slight haze appears on the glass slide. After 20 mins, the glass slides were sprinkled with water until the haze was gone and the slide became crystal clear. In order to test hydrophobicity, a droplet of water was dropped onto the hydrophobic glass surface. Due to hydrophobicity of the surface, water droplets rolled around the surface instead of spreading out, which is in good agreement with previous reports with the water droplet dynamics in literature precedent. ${ }^{166}$

\subsubsection{Synthesis of $20 \mathrm{~nm}$ average diameter citrate-stabilized gold nanospheres}

Gold nanospheres with an average diameter of $20 \mathrm{~nm}$ were synthesized using an established literature procedure. ${ }^{167}$ Briefly, $0.5 \mathrm{~mL}$ of $10 \mathrm{mM} \mathrm{HAuCl} \mathrm{H}_{4} \cdot 3 \mathrm{H}_{2} \mathrm{O}$ 
solution was added to $17.0 \mathrm{~mL}$ of nanopure water and heated to boiling. $2.5 \mathrm{~mL}$ of a $10 \mathrm{mM}$ trisodium citrate solution was then quickly added to the reaction mixture, and the solution was stirred for $10 \mathrm{~min}$. Finally, the resulting solution was stirred for another $15 \mathrm{~min}$ at room temperature and allowed to cool. A bright-red color indicated the formation of $20 \mathrm{~nm}$ average gold nanoparticles.

\subsubsection{Functionalization of $20 \mathrm{~nm}$ gold nanospheres with oligonucleotides}

In order to synthesize DNA-coated nanoprobes, $20 \mathrm{~nm}$ average gold nanoparticles were functionalized with a 21 base pair long oligonucleotide sequence (DNA-A") following literature precedent. ${ }^{111}$ Prior to the functionalization of Au nanoparticles, thiolated oligonucleotide was treated with $0.1 \mathrm{M}$ dithiothreitol (DTT) in disulfide cleaving buffer (0.17 M, pH 8). Then, oligonucleotide solution was purified from DTT using a desalting column (Nap-25, DNA grade, GE Healthcare). Briefly, $1 \mathrm{~mL}(1 \mu \mathrm{M})$ of purified thiolated DNA was added to $1 \mathrm{~mL}$ of $10 \mathrm{nM}$ gold nanoparticles and allowed to react while shaking $\left(1100 \mathrm{rpm}, 22^{\circ} \mathrm{C}\right.$ for 30 min to 1 hour using an Eppendorf Thermomixer. The particle and DNA solutions were then brought to $0.01 \%$ SDS (sodium Dodecyl sulfate) and $0.01 \mathrm{M}$ sodium phosphate buffer. Then, particle solutions were slowly treated with $\mathrm{NaCl}$ and sequentially bought to $0.05 \mathrm{M}, 0.1 \mathrm{M}, 0.2 \mathrm{M}$, and $0.3 \mathrm{M}$ with 30 min between each addition. After the final salt aliquot addition, the particle dispersion was allowed to sit overnight. The next day, the particle solution was centrifuged 3-4 times for 20 mins at a speed of $2000 \mathrm{~g}$ to remove the unreacted oligonucleotide and the pellet was resuspended in 20x TE buffer or NANOpure water. 


\subsubsection{Face-to-face stacking of nanoprisms}

$200 \mu \mathrm{L}$ of 2.4 OD DNA-A' functionalized nanoprisms were mixed with 200 $\mu \mathrm{L}$ of 2.4 OD DNA-A coated nanoprisms in a DNA hybridization buffer $(0.01 \mathrm{M}$ Phosphate buffer, $\mathrm{pH} 7.4+0.3 \mathrm{M} \mathrm{NaCl})$. The mixture was then heated to $60-65^{\circ} \mathrm{C}$, followed by slow cooling to room temperature for efficient DNA-mediated interactions between nanoprisms, leading to the formation of 1D face-to-face assemblies of nanoprisms. Scheme 2.1 shows the schematics of face-to-face arrangement of nanoprisms into 1D stacks.

Scheme 2.1: Schematics of face-to-face lamellar arrangement of gold nanoprisms induced by DNA hybridization.

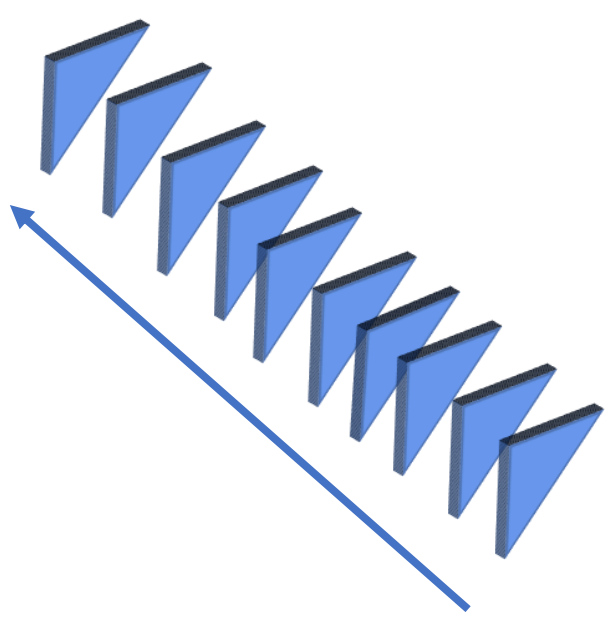




\subsubsection{Melting analysis of 1D nanoprisms stacks}

The melting analysis of 1D nanoprism stacks was monitored with an Agilent Cary 100 UV-Vis spectrometer (Agilent Technologies, Santa Clara, CA) equipped with a Perkin-Elmer PTP-1 Peltier Temperature Programmer, following literature

Scheme 2.2: Schematics of 1D gold nanoprism stacks during melting analysis.
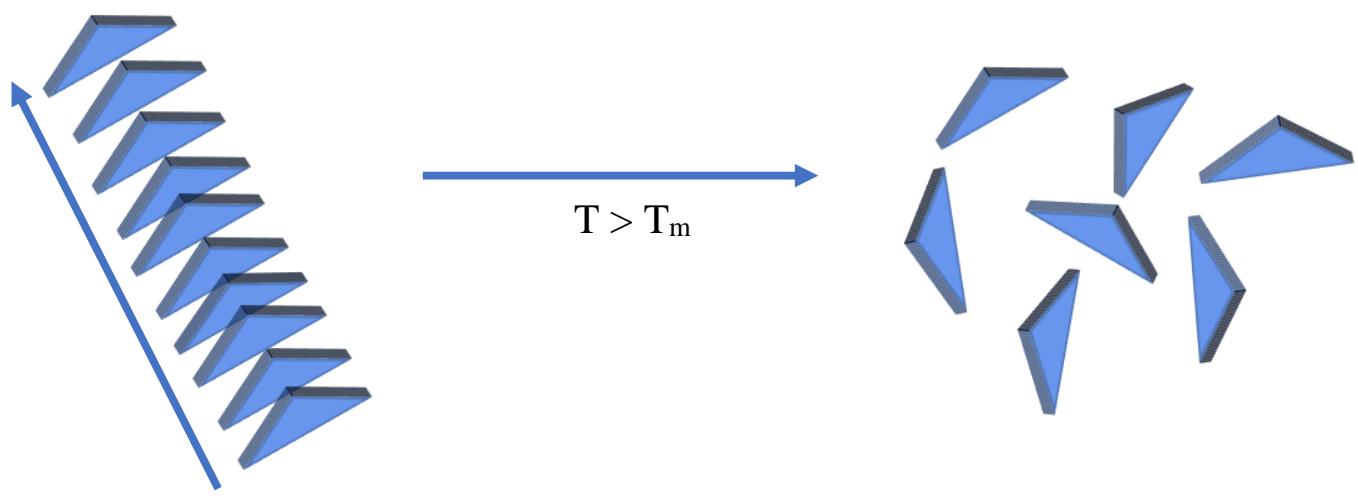

precedent. ${ }^{24}$ A melting profile was obtained by monitoring the change in absorbance of the predominant surface plasmon resonance (SPR) band of the gold nanoprisms with the change in temperature. Briefly, a $400 \mu \mathrm{L}$ dispersion of 1D nanoprism stacks was diluted in a $1.2 \mathrm{~mL}$ solution with the DNA hybridization buffer and loaded in a capped cuvette with a stir bar. The solution was then heated from $25^{\circ} \mathrm{C}$ to $80^{\circ} \mathrm{C}$ under continuous stirring at a ramp rate of $0.25^{\circ} \mathrm{C} / \mathrm{min}$. The melting temperature of the conjugates was determined by taking the first derivative of the temperature curve. 
Above the DNA melting temperature, the double helical structure of complementary DNAs denatures, which causes particle dispersion as shown in Scheme 2.2 and hence increases the absorbance of the SPR band of gold nanoprisms. Melting analysis of a control experiment was carried out by heating uncoated nanoprisms in the DNA hybridization buffer under similar conditions.

\subsubsection{Formation of 3D nanoprism superlattices}

In order to assemble 1D nanoprism stacks into 3D superlattices, the solution of 1D nanoprisms stacks was spun down and resuspended in 250-300 $\mu \mathrm{L}$ phosphate buffer to allow for a concentrated solution of 1D nanoprism stacks. The concentrated dispersion of 1D nanoprisms stack was then annealed at a temperature $1-3^{\circ} \mathrm{C}$ below the melting temperature of $1 \mathrm{D}$ stacks for $1-3 \mathrm{hrs}$. Briefly the solution was heated to and annealed at $65^{\circ} \mathrm{C}, 66^{\circ} \mathrm{C}$ and $67.5^{\circ} \mathrm{C}$ for several hrs.

Dark-field microscopy was used to monitor the crystal growth at each temperature. While annealing, $5 \mu \mathrm{L}$ of solution was quickly taken from the capped cuvette and drop cast on a glass slide before placing a coverslip over the sample for dark-field microscopy imaging.

\subsubsection{Surface modification of carboxylate modified polystyrene (PS) beads}

Carboxylate modified PS beads can be covalently coupled with aminecontaining protein, antibody or any other biomacromolecules including oligonucleotides using carbodiimide chemistry. The typical covalent coupling of carboxylate modified PS beads is depicted in Scheme 2.3. 
Scheme 2.3: Schematics of covalent coupling between carboxylate modified PS beads and amine functionalized ligands through carbodiimide chemistry.

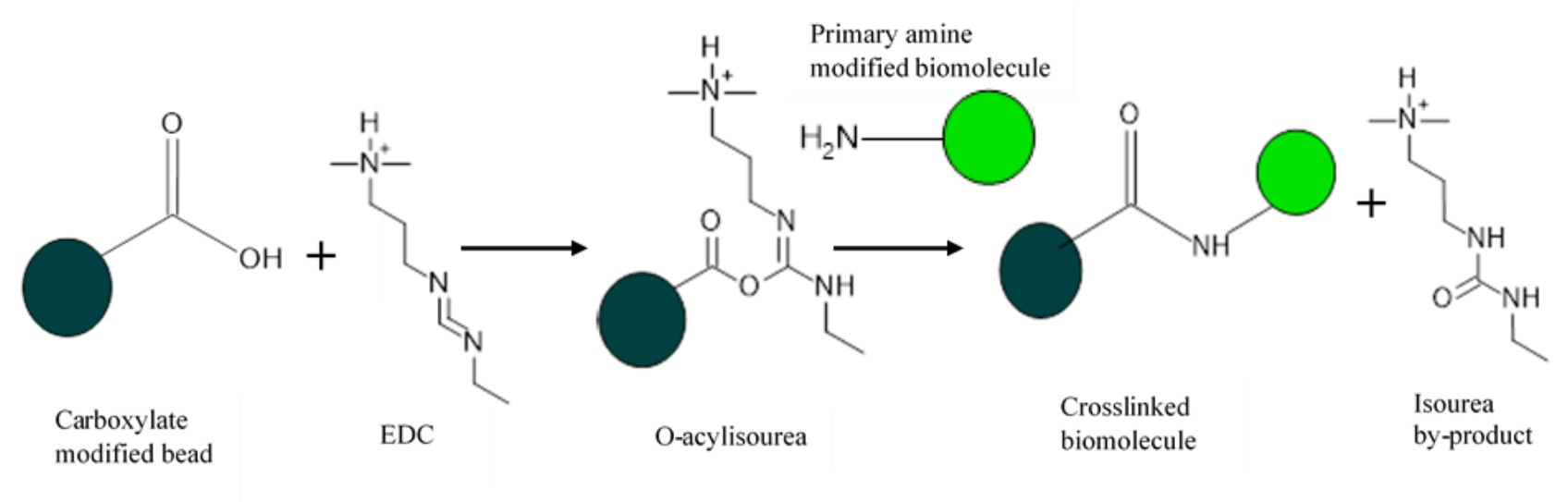

EDC (1-ethyl-3-(3-dimethylaminopropyl) carbodiimide) reacts with carboxylic acid groups on the beads to form an active O-acylisourea intermediate. It is then displaced by nucleophilic attack from primary amino groups of ligands (DNA/antibody/protein) in the reaction mixture. An amide bond is formed between the primary amine forms and the original carboxyl group. The O-acylisourea intermediate is unstable in aqueous solutions. To increase stability of the intermediate, EDC couples NHS to carboxyls, leading to formation of an NHS ester that is considerably more stable than the O-acylisourea intermediate. The chemical structures of Sulfo-NHS and EDC are shown in Figure 2.1 and 2.2 respectively. 


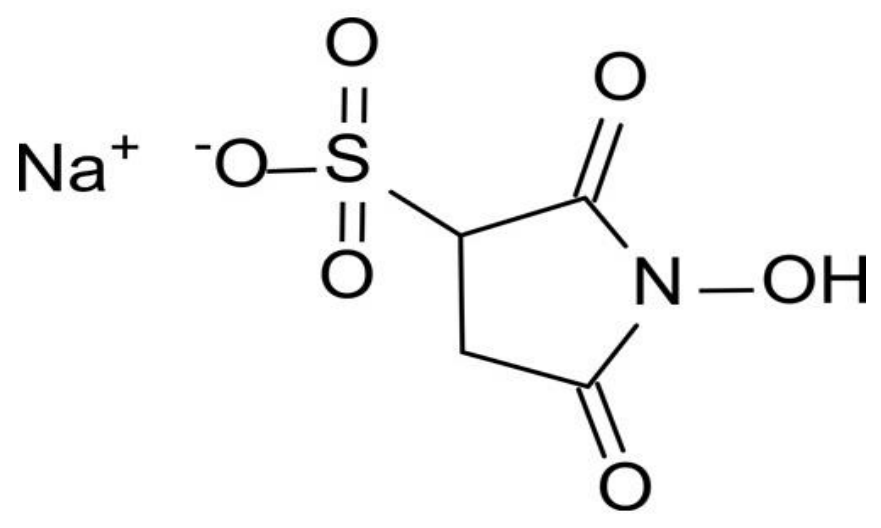

Figure 2.1: Chemical structure of Sulfo-NHS ((N-hydroxysulfosuccinimide).

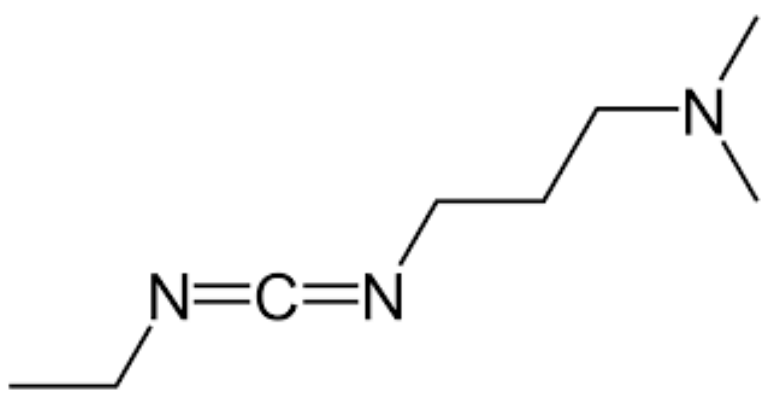

Figure 2.2: Chemical structure of EDC (1-ethyl-3-(3-dimethylaminopropyl) carbodiimide). 
Carboxylate modified PS beads were covalently linked with amine modified DNA-A' strands through carbodiimide chemistry. First, PS microparticles were purified following an established latex bead purification protocol. ${ }^{168}$ Briefly, 2.5 $\mathrm{ml}(40 \mathrm{mg} / \mathrm{ml})$ latex microspheres was taken in a $15 \mathrm{~mL}$ centrifuge tube and diluted with $10 \mathrm{ml} 50 \mathrm{mM}$ MES buffer ( $\mathrm{pH} 6.1$ ). The mixture was centrifuged at $\sim 3,000 \mathrm{~g}$ for 20 min to sediment particles, the supernatant was removed, and the pellet was redispersed in $10 \mathrm{~mL}$ MES buffer. The process was repeated three times. The final suspension was in $5 \mathrm{~mL}$ MES buffer to create a $20 \mathrm{mg} / \mathrm{mL}$ particle suspension. Then, the PS bead solutions were sonicated using an ultrasonic convertor operated at low power (5 watts) to disperse the beads.

Next, PS beads were incubated with $0.1 \%$ polyethylene glycol $(6000 \mathrm{MW})$ for 3 hours at room temperature in order to block hydrophobic regions on the PS beads and prevent non-specific adsorption of coating reagents. After 3 hours of incubation, beads were centrifuged at $3000 \mathrm{rpm}$ for 20 mins (3 times) and redispersed in MES buffer ( $\mathrm{pH}$ 6.1). Then, purified carboxylate modified beads were coupled with amine functionalized oligonucleotide (DNA-A') by either a one step or a two-step procedure. The one step method is described as follows: $1 \mathrm{~mL}$ of purified latex bead (1\%) was placed in a $15 \mathrm{~mL}$ tube with $10 \mathrm{mg} / \mathrm{mL}$ freshly prepared EDC (1-ethyl-3-(3-dimethylaminopropyl) carbodiimide) solution in MES buffer. The mixture was kept on a rotatory shaker for 5 mins for thorough mixing. Then $3 \mathrm{~mL}$ of DNA-A' with 2 OD (optical density) concentration was added to the mixture. Finally, $10 \mathrm{mg}$ sulfo-NHS (N-hydroxysulfosuccinimide) was added to the 
mixture and the mixture was incubated at room temperature overnight on a rotary shaker for gentle mixing. The next day the solution mixture was centrifuged at $2000 \mathrm{rpm}$ for 10 mins and washed with $0.01 \mathrm{M}$ phosphate buffer 3 times to remove unbound oligonucleotide and the final pellet was stored at $4{ }^{\circ} \mathrm{C}$.

The two-step procedure involves the activation of the carboxylates on the beads' surface in the first step and addition of DNA-A' in the second step. Briefly, $1 \mathrm{~mL}$ of purified latex beads (1\%) was placed in a $15 \mathrm{~mL}$ centrifuge tube with 10 $\mathrm{mg} / \mathrm{mL}$ freshly prepared EDC (1-ethyl-3-(3-dimethylaminopropyl) carbodiimide) solution in MES buffer (pH 6.1). $10 \mathrm{mg}$ sulfo-NHS (N-hydroxysulfosuccinimide) was added to the mixture and the mixture was incubated at room temperature for 3 hours with constantly shaking, followed by 3 centrifugal washing with $50 \mathrm{mM}$ MES buffer (pH 6.1) to remove extra EDC and sulfo-NHS. In the second step, 1 mL of DNA-A' with 2 OD (optical density) concentration was added to the mixture and incubated overnight at room temperature while shaking on a rotary shaker after gentle mix. Next day the solution was centrifuged at $2000 \mathrm{rpm}$ for $10 \mathrm{mins}$ and washed with phosphate buffer 3 times to remove unbound oligonucleotide and the final pellet was stored at $4^{\circ} \mathrm{C}$ for future use.

A high density of DNA grafting is crucial to maintain colloidal stability and prevent non-specific interactions. ${ }^{169}$ While DNA-A' functionalization via two-step conjugation process produced well dispersed PS beads, one step conjugation process led to some random aggregation of beads despite less centrifugal washes involved in one step process. This indicates that two-step process is more efficient 
than one step process for DNA functionalization of PS beads through EDC coupling.

\subsubsection{Coating of gold nanoprisms onto PS beads}

$25 \mu \mathrm{L}$ of 1.2 OD DNA-A' functionalized PS beads were added to $100 \mu \mathrm{L}$ of 1.8 OD DNA-A coated nanoprisms in a DNA hybridization buffer $(0.01 \mathrm{M}$ Phosphate buffer, $\mathrm{pH} 7.4+0.1 \mathrm{M} \mathrm{NaCl})$. The mixture was then heated to $55^{\circ} \mathrm{C}$ followed by slow cooling to room temperature. The mixture was then diluted to $400 \mu \mathrm{L}$ using DNA hybridization buffer for optical characterization. In order to assess the effect of salt concentration on the DNA mediated binding event between nanoprisms and PS beads, the salt concentration was varied from $0.1 \mathrm{M}$ to $0.2 \mathrm{M}$ and then $0.3 \mathrm{M} \mathrm{NaCl}$, while keeping the concentrations of nanoprisms and PS beads unchanged.

\subsubsection{Melting analysis of PS bead/gold nanoprism conjugates}

The melting analysis of gold nanoprism-coated PS beads was monitored with an Agilent Cary $100 \mathrm{UV}$-Vis spectroscopy following literature precedent. ${ }^{170} \mathrm{~A}$ melting profile was obtained by monitoring the change in absorbance of the predominant surface plasmon resonance (SPR) band of the gold nanoprisms (895 $\mathrm{nm}$ ) with the change in temperature. Briefly, $100 \mathrm{uL}$ of 1.2 OD DNA-A' coated PS beads was hybridized with $400 \mathrm{uL}$ of DNA-A coated gold nanoprisms. The conjugates were then diluted with DNA hybridization buffer and taken in a capped

cuvette with a stir bar. The mixture was then heated from $45-90^{\circ} \mathrm{C}$ at a ramp rate of 
$0.25^{\circ} \mathrm{C} / \mathrm{min}$. The melting temperature of the conjugates was determined by taking the inflection point of the temperature curve. Melting analysis of a control experiment was carried out by mixing uncoated nanoprisms and uncoated PS beads in a DNA hybridization buffer and heating the mixture under similar conditions.

\subsubsection{Formation of 3D PS beads/gold nanoprism aggregates}

PS beads/nanoprism conjugates at $0.3 \mathrm{M} \mathrm{NaCl}$ were heated to $60{ }^{\circ} \mathrm{C}$, which is above the $\mathrm{T}_{\mathrm{m}}$ of the DNA linker $\left(41^{\circ} \mathrm{C}\right)$ and below the $\mathrm{T}_{\mathrm{m}}$ of PS beads/nanoprisms conjugates $\left(78^{\circ} \mathrm{C}\right)$, followed by slow cooling. The long-range interaction of DNA-A coated beads and DNA-A coated nanoprisms led to the formation of a 3D ensemble of nanoprisms immobilized beads.

\subsubsection{Synthesis of gold nanoprism dimers and trimers}

A schematic for the synthesis of gold nanoprism dimers and trimers is given in Scheme 2.4. Two sets of Janus nanoprisms, PEG | DNA-A and PEG | DNA-A', where 'l' denotes coatings are on opposite sides of the nanoprisms, were synthesized using the protocol described in section 2.3.2. Then, $200 \mu \mathrm{L}$ of $0.6 \mathrm{OD}$ dispersions of Janus nanoprisms were mixed in a 1:1 ratio in a DNA hybrization buffer $(0.01 \mathrm{M} \mathrm{PB}+0.1 \mathrm{M} \mathrm{NaCl})$. The mixture was then heated to $60^{\circ} \mathrm{C}$ and allowed to slowly cool down to ambient temperature. For trimer synthesis, DNA-A | DNA-A nanoprisms (200 $\mu \mathrm{L}$ of 0.6 OD) was mixed with PEG | DNA-A' nanoprisms (100 $\mu \mathrm{L}$ of $0.6 \mathrm{OD}$ ) in a 2:1 ratio in hybridization buffer and heated to $60^{\circ} \mathrm{C}$ followed by slow cooling. 
Scheme 2.4: Schematics of gold nanoprism dimer and trimer formation
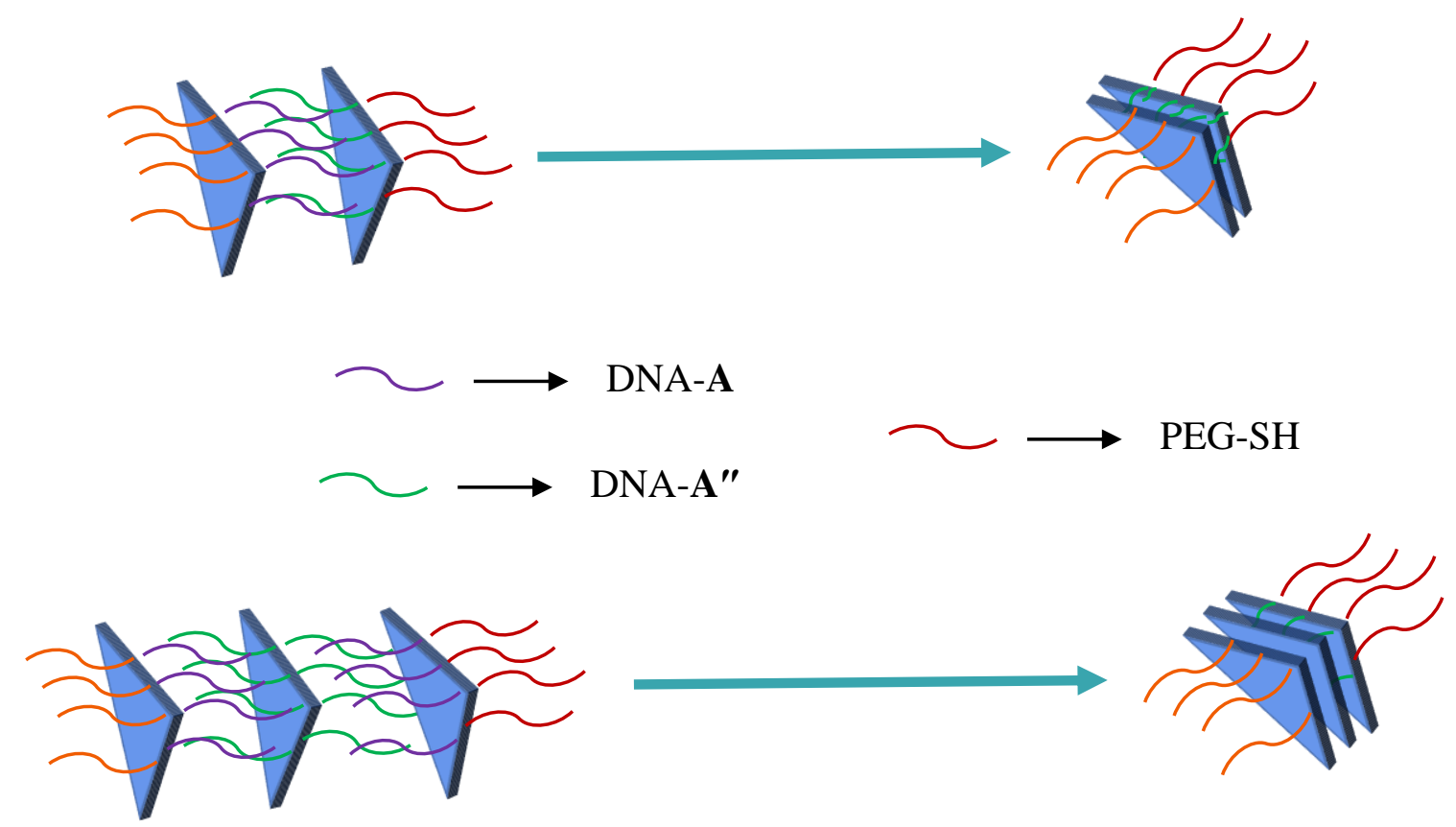

\subsection{Instrumentation}

\subsubsection{UV-Vis spectroscopy}

UV absorption spectra were obtained with a UV Visible Spectrometer (Varian Cary 50 BIO UV, McKinley Scientific, Sparta, NJ) using either a plastic cuvette of $1 \mathrm{~mL}$ sample volume or a quartz cuvette of $400 \mu \mathrm{L}$ sample volume. UV-Vis measurements were carried out to characterize synthesis and surface functionalization of gold nanoprisms as well as various DNA-mediated assemblies 
of nanoprisms. Melting analyses were performed on an Agilent Cary UV-Vis 100 spectrometer (200-900nm) equipped with a Perkin-Elmer PTP-1 Peltier Temperature Programmer.

\subsubsection{Scanning electron Microscopy}

Scanning electron microscopy (SEM) and scanning tunneling electron microscopy (STEM) were performed at different magnifications using a Carl Zeiss SMT AG SUPRA (Carl Zeiss AG, Oberkochen, Germany) 35VP field S-6 emission scanning electron microscope (FESEM) operating at an accelerating voltage of 3$15 \mathrm{kV}$ for SEM depending on the sample type and $20-25 \mathrm{kV}$ for STEM, and using SE2 secondary electron detector or Inlens detector and STEM detector.

\subsubsection{Sample preparation for SEM/STEM using TEM grid}

SEM samples were prepared in two ways. First, $10 \mu \mathrm{L}$ of sample was drop cast on a TEM grid and quickly taken out. This process was carried out 6-8 times. A control experiment was also carried out by drop casting just bare nanoprisms on the TEM grid. No drying effect was observed. Second, samples were drop-cast on the substrate, which was followed by slow removal of solvent using a filter paper after 5 mins of drop casting.

\subsubsection{Sample preparation for SEM on glass slide}

$10 \mu \mathrm{L}$ of sample was drop cast on a marked area of an APTES coated silica glass slide and solvent was evaporated using in a stream of lab air for 10 mins. 
Double-sided carbon tape was applied around the sample position in a rectangular fashion to mitigate charging issues. Using a low acceleration voltage in the range of 2-3 KV and changing the aperture to $10-20 \mu \mathrm{M}$, SEM imaging of nanoprisms and other nanostructures with spatial resolution of 20-200 nm was accomplished.

\subsubsection{Dynamic Light Scattering (DLS) size and zeta potential measurements}

Hydrodynamic diameter and zeta potential measurements were accomplished using a Zetasizer (Zetasizer Nano ZS90, Malvern Instruments Ltd., Westborough, MA). The instrument is equipped with a $633 \mathrm{~nm}$ laser source, and a backscattering detector at $173^{\circ}$. For DLS size measurements, either plastic cuvettes of $1 \mathrm{~mL}$ sample volume or quartz cuvette of $400 \mu \mathrm{L}$ were used and SOPs for both simple and complex solvents were created for the relevant sample. In case of simple solvent, either water/ 0.01M PBS was selected and the corresponding refractive index was incorporated while for complex solvent, both water/0.01M PBS and $0.3 \mathrm{M} \mathrm{NaCl}$ were selected as solvents and their refractive indices were incorporated. DLS measurements were carried out in triplet, each consisting of two runs. Each run was repeat 10 times.

\subsubsection{Fluorescence measurements}

Fluorescence measurements of yellow-fluorescent polystyrene latex beads were carried out on a SpectraMax M2 plate reader using a fluorescence quartz cuvette of $700 \mu \mathrm{L}$ sample volume. The fluorescence spectra were collected in the 
range of 400-600 nm using a $470 \mathrm{~nm}$ excitation wavelength. Excitation was fixed at $470 \mathrm{~nm}$ while emission was allowed to sweep at a step size of $20 \mathrm{~nm}$.

\subsubsection{Darkfield microscopy}

Dark field microscopy and hyperspectral imaging were performed on a research grade optical microscope (Olympus BX43) equipped with an integrated hyperspectral analysis system (CytoViva Inc., Auburn, AL, USA). This system uses a halogen lamp light source and a darkfield oil condenser (NA 1.20-1.40) for sample illumination. High resolution darkfield images were obtained using a Dage camera (model XLMCT, Dage-MTI, Michigan City, IN, USA). Hyperspectral profiles are acquired using a Pixelfly camera and visualized using Environment for Visualizing Images (ENVI) 4.8 software (Exelis Visual Information Solutions, Boulder, CO, USA).

\subsubsection{Acquisition of single particle scattering profiles}

For collecting the scattering profile of monomer gold nanoprisms, a diluted DNA-A coated gold nanoprism solution was drop cast on a glass slide that had been etched with marker system containing numbers etched on the glass slide by photolithography (donated from the Harnett Lab at the University of Louisville.

First, darkfield images were collected using a 100x objective. Then hyperspectral imaging was collected on the same area of sample using the same magnification and at an acquisition time $0.25 \mathrm{~s}$. Next, a Z-profile of the particle sample was collected with average scan window width of 3 units. In order to compensate for 
the effect of camera optics and spectrograph on the spectral recording, spectral normalization was performed. In order to perform spectral normalization, the spectrum of the light source was obtained by imaging blank glass slides under the same settings the samples analyzed through the ENVI software. Then, the value of the highest point on the blank spectrum was set to unity by this expression: float ( 1 (/max 1$)$. The spectrum of the desired specimen was corrected for instrumental effects by dividing the specimen spectrum by the normalized spectrum, which was carried by implementing this correction expression: float (S1) and float (S2), where $\mathrm{S} 1$ is specimen spectrum and $\mathrm{S} 2$ is normalized lamp spectrum.

\subsubsection{Surface Enhanced Raman Scattering Experiments}

Raman and photoluminescence (PL) analyses were carried out using a Renishaw InVia micro-Raman/PL system (Renishaw, Wotton-Under-Edge, U.K.) equipped with a $633 \mathrm{~nm}$ HeNe laser with an operational range $100-2000 \mathrm{~cm}^{-1}$ for Raman and 500-800 nm for PL measurement.

All the Raman spectra were recorded under the same ambient conditions: 50x microscope objective with a laser spot size of $1 \mu \mathrm{m}, 10 \mathrm{~s}$ data acquisition time and $150 \mathrm{~mW}$ laser power. To evaluate the SERS performance of the 3D nanoprism superlattices, $10.0 \mu \mathrm{L}$ of probe molecule $\mathrm{MB}$ at different concentrations (ranging from $10^{-3} \mathrm{M}$ to $10^{-10} \mathrm{M}$ ) was drop cast on $3 \mathrm{D}$ nanoprism superlattices on a glass slide and kept in a fume hood until dry. Raman spectra were then collected from at least 6 different locations on the sample area. 


\subsubsection{Photoluminescence measurements}

The PL measurements were carried out in solution on a glass slide with a cover slip at room temperature using a Renishaw Invia Raman/PL system. The excitation source was a HeNe red laser emitting at $633 \mathrm{~nm}$. The PL emissions spectra were collected from the wavelength range of $500 \mathrm{~nm}$ to $800 \mathrm{~nm}$ by a Charge-couple device (CCD) detector.

A $5 \mu \mathrm{L}$ solution of nanoprism superlattices was deposited on a predetermined position in a clean glass slide and allowed to airdry. Then $5 \mu \mathrm{l}$ of $0.1 \mu \mathrm{M}$ of the dye solution was drop cast in the same position of the glass slide and a cover slip was placed over the sample droplet. 


\section{CHAPTER 3}

\section{FACET SELECTIVE ASYMMETRIC FUNCTIONALIZATION OF ANISOTROPIC GOLD NANOPRISMS FOR JANUS PARTICLE SYNTHESIS}




\subsection{Introduction}

The quest for attaining the capability to construct tailored nanostructure and devices for given applications and with controllability at the atomic and molecular level has driven a growing interest in creating new functional building blocks for selfassembly of supramolecular constructs. ${ }^{171-172}$ One of the grand challenges in selfassembly is to generate colloidal building blocks with multiple distinct chemical reactivities within the same particles from a monophasic solvent. ${ }^{173}$ In this regard, colloidal Janus particles which possess asymmetric structures and/or multiple surface functionalities have emerged as a subject of intense interest due to their capability of multiple distinct interactions with their environment. ${ }^{57,174}$ The asymmetric nature of Janus particles enables directional interactions or facet selective reactivity, which are otherwise impossible to achieve in their isotropic counterparts. ${ }^{175}$ Directional binding capabilities along with shape anisotropy make these Janus particles attractive building blocks for numerous applications in different fields, such as catalysis, ${ }^{176}$ drug release, ${ }^{177}$ displays, ${ }^{178}$ sensors, ${ }^{179}$ water purification ${ }^{180}$ waterproof coatings,,${ }^{180}$ surfactants, ${ }^{181}$ self-propelled carriers, and microprobes or sensors. ${ }^{182}$ In particular, amphiphilic Janus particles are conceptually similar to molecular amphiphiles found in other complex molecular systems such as phospholipids in cell membranes,${ }^{56}$ and diblock copolymers. ${ }^{183}$

The synthesis of Janus particles typically requires complex functionalization techniques such as emulsions, ${ }^{184}$ interfacial reactions, ${ }^{185}$ metal evaporation, ${ }^{186}$ biphasic electrodynamic jetting, ${ }^{187}$ and microfluidics ${ }^{178,188}$ to impart shape anisotropy and 
directional interaction capabilities to the core particles. While these synthetic strategies allow one to rationally design different types of Janus particles synthesis, they have been mostly limited to spherical core particles and may not be applicable for anisotropic core particles.

Although numerous studies have been performed for the preparation of Janus particles from isotropic cores, there have been only a handful of instances concerning non-spherical Janus particle synthesis reported in the literature. For example, Walther, et. al. developed a novel template assisted synthetic approach for the preparation of sheet and disk type Janus particles comprising a crosslink-able central polybutadiene layer of a triblock terpolymers such as polystyrene-block-polybutadiene-blockpolybutadiene (methyl methacrylate) (PS-b-PB-b-PMMA) (SBM) and two different outer sides of polystyrene and poly(tert-butyl methacrylate). ${ }^{189}$ Moreover, Liu, et. al. demonstrated a novel approach to fabricate linear, branched, and cyclic assemblies of gold nanorods and their structural isomers via a solvent controlled step-growth polymerization of thiol modified polystyrene preferentially attached to the two ends of gold nanorods by ligand exchange. ${ }^{190}$ The synthesis of anisotropic shape Janus particles such as biodegradable bicompartmental discoid, and rod-shaped microparticles has also been accomplished by electrohydrodynamic co-jetting of poly(lactide-co-glycoside) polymer solutions in organic solvent. However, because of the larger dimeter of the fluidic channels of the co-jetting device, this method can produce only particles larger than $1 \mu \mathrm{m} .{ }^{191}$ 
Here we describe a facile method for stepwise and controllable functionalization of the two major surfaces of anisotropic gold nanoprisms to synthesize Janus nanoparticles from an anisotropic core particle. Using hexadecane thiol (HexaD) and thiolated DNA as surface coatings, we synthesized amphiphilic Janus nanoprisms that dynamically align themselves at the interface of water-chloroform mixture, despite being dispersible in water, and readily adsorb to both hydrophilic and hydrophobic surfaces. We also employed thiolated poly(ethylene) glycol (PEG) and thiolated DNA to synthesize a second set of Janus nanoprisms that undergo facet selective asymmetric assembly. ${ }^{165}$

\subsection{Result and Discussion}

\subsubsection{Synthesis of Janus gold nanoprisms}

Synthesis of Janus nanoprisms was accomplished through a controllable coating mechanism (Scheme 3.1) consisting of three steps:

\section{Step 1. Creating a positively charged surface for a monolayer deposition of negative charged nanoprisms.}

Silica glass slides were functionalized with (3-Aminopropyl) triethoxysilane (APTES) to create a positively charged surface (see section 2.3 .7 for details) Nanoprisms were deposited on the APTES surface via drop-casting for 5-10 minutes, depending on the optical density (OD) of the nanoprisms suspension so that a monolayer of nanoprisms was formed. Specifically, $1 \mathrm{~mL}$ of an aqueous suspension of nanoprisms with 8-10 OD was deposited on the APTES surface for 
10 mins. Then extra and unbound nanoprisms were taken out from the APTES surface, followed by a thorough wash with water and sonication for 10 mins to remove loosely bound nanoprisms from APTES surface.

Scheme 3.1: Schematics for the synthesis of Janus gold nanoprisms.

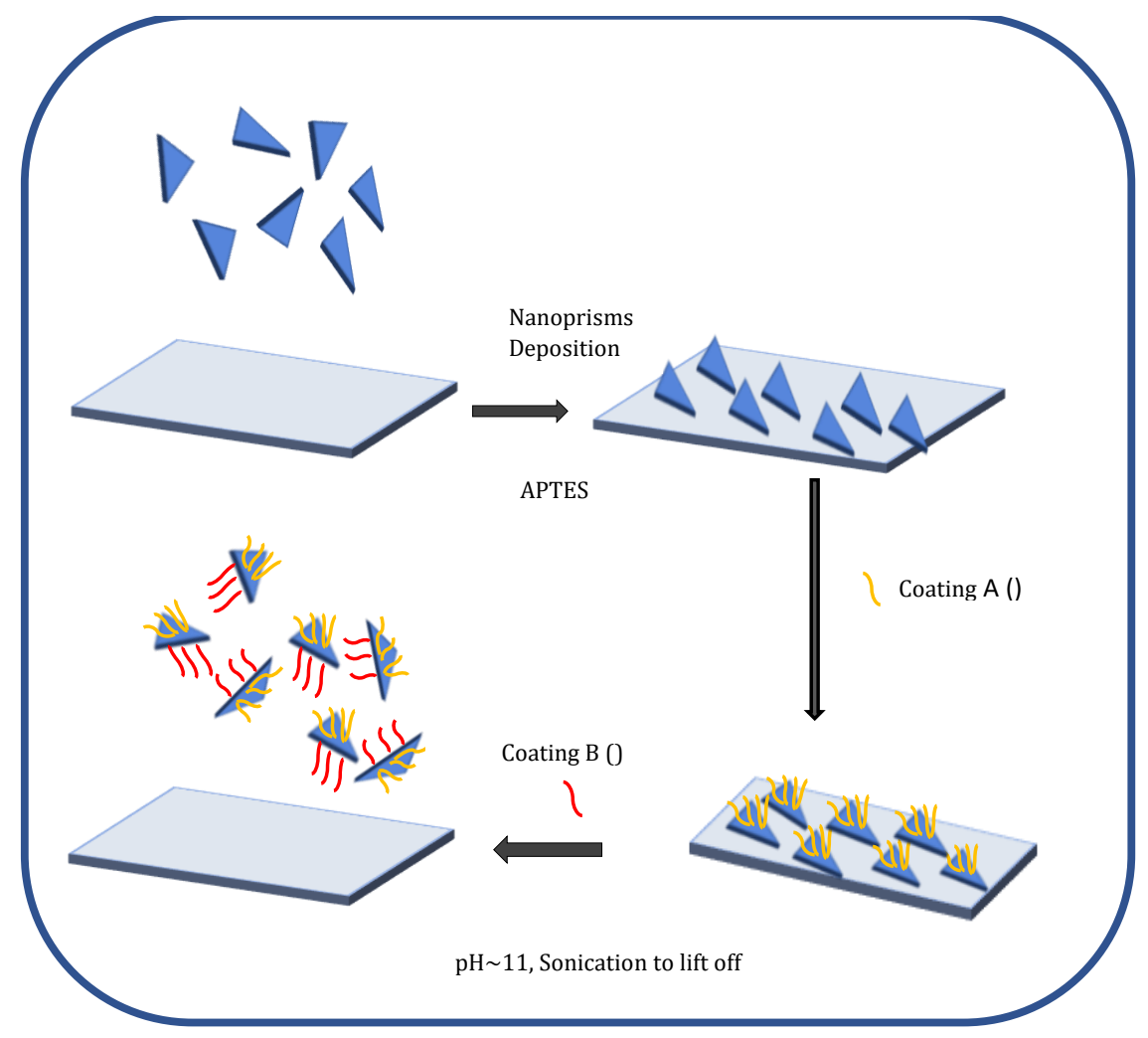

\section{Step 2. Application of first coating molecules on the exposed facet of nanoprisms}

The exposed nanoprisms facets were functionalized with either hexadecane thiol (HexaD) or thiolated poly(ethylene) glycol (PEG-SH)). In order to accomplish 
this, glass slides with a monolayer of nanoprisms were taken in either ethanolic solution of $1 \mu \mathrm{M}$ of HexaD or a $100 \mu \mathrm{M}$ PEG-SH solution in water for $3 \mathrm{hrs}$. Then the glass slides were removed from the coating solutions and excess coating molecules were removed by sonication and rinsing with ethanol for HexaD and water for PEG-SH.

\section{Step 3. pH induced lifting-off of nanoprisms from the surface with sonication and application of the second coating on the other facet.}

Half-coated nanoprisms were then lifted off from APTES with 4-5 mild sonication in potassium carbonate at $\mathrm{pH} \sim 11$, and the uncoated facet was subsequently functionalized with thiol modified DNA (See section 2.3.2 for details)

\subsubsection{Characterization of Janus nanoprisms}

Nanoprisms were synthesized via DiaSynth process following literature precedent $^{52}$ and purified through centrifugation to remove gold pseudospherical nanoparticle (See section 2.3.1 for details). As shown in Figure 3.1, the UV-Vis absorption peak for pseudospherical nanoparticle at $540 \mathrm{~nm}$ completely diminished after purification, indicating the success of the purification procedure and the removal of pseudospherical nanoparticles from the mixture. 


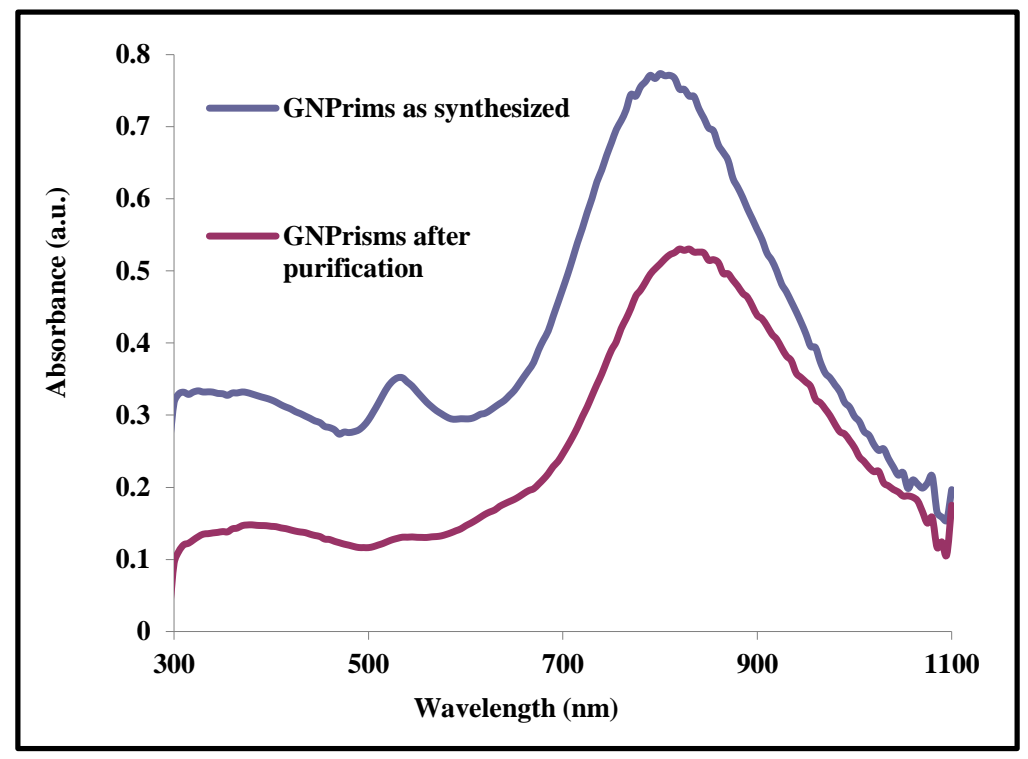

Figure 3.1: UV-vis spectra of nanoprisms before and after purification.

Amphiphilic HexaD | DNA-A and PEG | DNA-A (where '|' denotes coatings are on opposite sides of the nanoprisms) Janus nanoprisms were synthesized by applying HexaD or PEG as the first coating while nanoprisms were deposited on the APTES coated glass slide, and then a thiolated 21-mer adenosine-rich oligonucleotide (DNA-A ) strands as the second coating after lifting off from APTES surface. The success of coating of HexaD/PEG and DNA-A onto nanoprisms was confirmed by UV-vis absorption spectroscopy and zeta potential measurement (Figure 3.2 and Table 3.1). The surface plasmon resonance (SPR) maximum of nanoprisms is red shifted from $820 \mathrm{~nm}$ to $838 \mathrm{~nm}$ after HexaD functionalization on one facet of the nanoprisms and then further shifted to $843 \mathrm{~nm}$ 
after lifting-off and coating DNA-A on the other facet (Figure 3.2A). The SPR maximum of the nanoprisms experienced a larger red shift in transitioning from uncoated to half-coated HexaD nanoprisms compared to transition from half-coated HexaD nanoprisms to HexaD | DNA Janus nanoprisms.
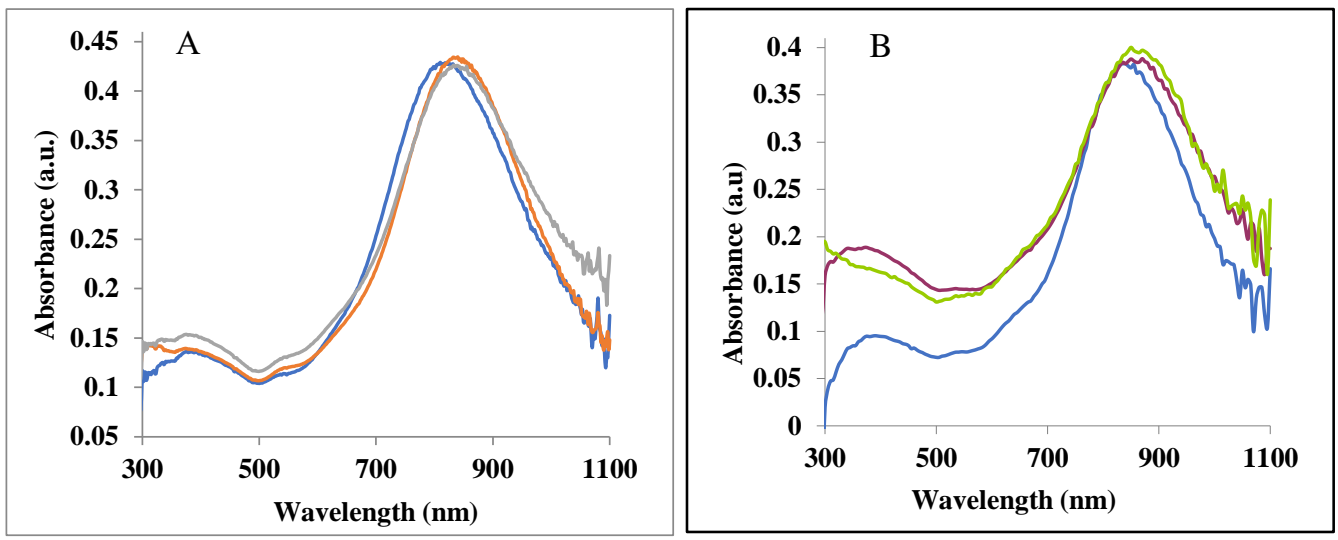

Figure 3.2: Normalized UV-vis spectra of A) uncoated nanoprisms (blue), half HexaD coated (orange) and HexaD | DNA-A Janus nanoprisms (gray), and B) Uncoated nanoprisms (blue), half PEG coated (green), and PEG | DNA-A nanoprisms (red).

This could be due to hydrophobic-hydrophobic interactions between neighboring nanoprisms after HexaD coating on one facet causing agglomeration of half-coated nanoprisms thereby affecting the perceived UV-visible spectrum.

The change in surface charge after modification of each major facet of nanoprisms was monitored by Zeta potential measurement. The Zeta potential of 
nanoprisms decreased from $-35.4 \pm 1.42 \mathrm{mV}$ (uncoated nanoprisms) to $-22.0 \pm 1.65$ $\mathrm{mV}$ for half-coated HexaD nanoprisms (Table 3.1).

Table 3.1: Surface Zeta potential of nanoprisms before and after functionalization.

\begin{tabular}{llll}
\hline Sample name & $\begin{array}{l}\text { Zeta potential, } \\
(\mathrm{mV})\end{array}$ & Sample name & $\begin{array}{l}\text { Zeta potential, } \\
(\mathrm{mV})\end{array}$ \\
& $\begin{array}{l}\text { Average } \pm \text { Std. } \\
\text { dev. }\end{array}$ & & $\begin{array}{l}\text { Average } \pm \text { Std. } \\
\text { dev }\end{array}$ \\
\hline Uncoated nanoprisms & $-35.4 \pm 1.42$ & Uncoated & $-35.7 \pm 3.06$ \\
HexaD | nanoprisms & $-22.0 \pm 1.65$ & nanoprisms & \\
HexaD | DNA1 Janus & $-33.7 \pm 2.14$ & PEG | Danoprisms & $-18.1 \pm 2.23$ \\
nanoprisms & & nanoprisms & $-28.5 \pm 1.56$ \\
\hline
\end{tabular}

Despite the presence of the hydrophobic HexaD coating on one facet and the decrease in zeta potential, half-coated HexaD nanoprisms remained dispersible in water. This is consistent with a similar study, which reported that gold nanoparticles with $60 \%$ hydrophobicity were still dispersible in water. ${ }^{192}$ Coating with DNA on the uncoated nanoprism facet stabilized Janus nanoprisms as evidenced by an increase of Zeta potential from to $-22.0 \pm 1.65 \mathrm{mV}$ to $-33.7 \pm 2.14$ mV, (Table 3.1 and Figure 3.3A) due to the negatively charged phosphate backbone of DNA-A. 

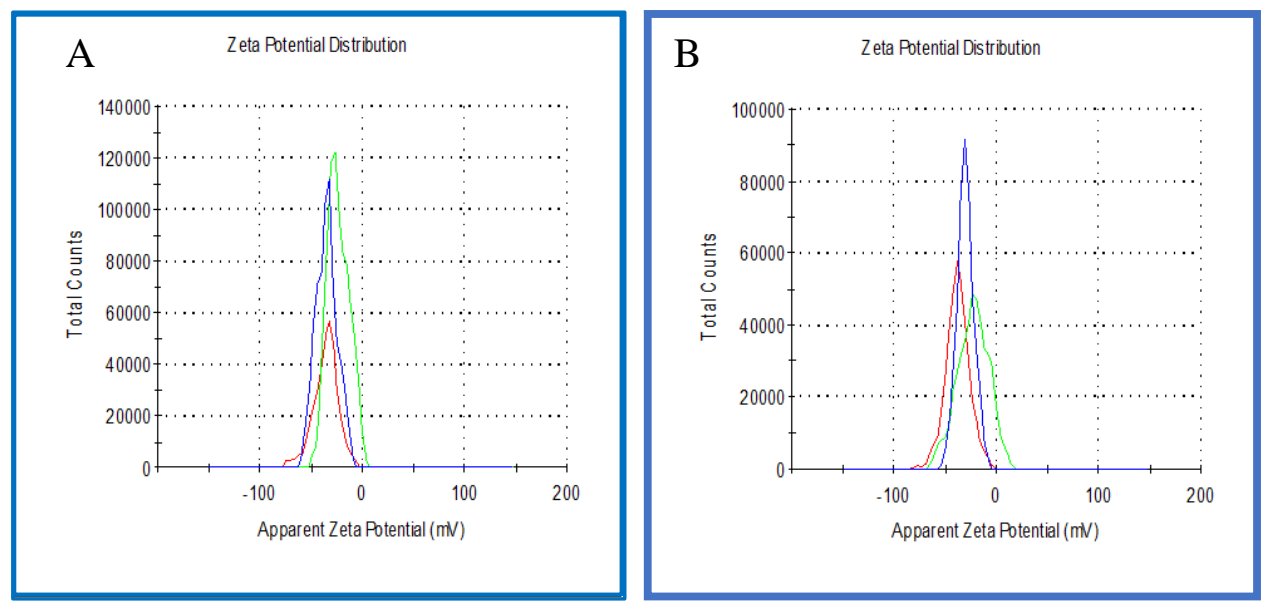

Figure 3.3: A) Representative Zeta Potential distribution graph of uncoated nanoprisms (red), HexaD | nanoprisms (Green), \& HexaD | DNA-A nanoprisms (blue), and B) representative Zeta Potential distribution graph of Uncoated nanoprisms (red), PEG $\mid$ nanoprisms (Green), \& PEG | DNA-A nanoprisms (blue).

On the other hand, the localized surface plasmon resonance band of the nanoprisms red shifted from $845 \mathrm{~nm}$ to $854 \mathrm{~nm}$ after first coating PEG on one facet of nanoprisms (Figure 3.2B). The SPR band was then further red shifted to $860 \mathrm{~nm}$ after the application of DNA-A coating on the other facet. The success of coating was also confirmed by the change in particle Zeta potential upon successive coating events. The Zeta potential of nanoprisms expectedly dropped from $-35.7 \pm 3.06 \mathrm{mV}$ to $-18.1 \pm 2.23 \mathrm{mV}$ after PEG coating due to shielding of the nanoprisms surface charge by the non-ionic surface coating. This is consistent with a previous study, which showed that PEG-SH significantly decreases the surface charge of bare gold nanoparticles. ${ }^{193}$ The Zeta potential of the half-coated PEG nanoprisms increased to 
$28.5 \pm 1.56 \mathrm{mV}$ after DNA-A coating, (Table 3.1 and Figure 3.3B), attributable to the negatively charged phosphate backbone of DNA-A .

\subsubsection{Amphiphilicity of Janus HexaD | DNA-A gold nanoprisms}

In order to corroborate the dual nature and prove amphiphilicity of the Janus nanoprisms, Janus HexaD | DNA-A , HexaD | HexaD, and DNA-A | DNA-A nanoprisms were placed in three separate vials containing water layered on chloroform, Figure 3.4.

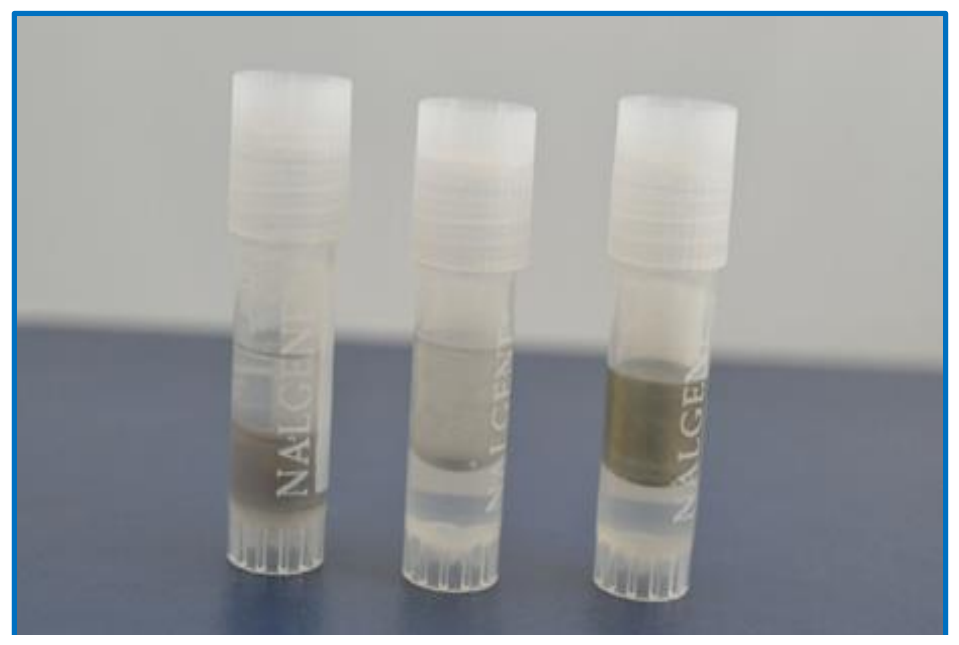

Figure 3.4: Amphiphilic Janus nanoprisms in a dual-phase water/chloroform system. Left: HexaD | HexaD nanoprisms, Middle: HexaD | DNA-A Janus, and Right: DNA-A | DNA-A nanoprisms.

HexaD | HexaD nanoprisms (Figure 3.4 left) dispersed in the chloroform layer due to the hydrophobicity of the HexaD coating. Conversely, DNA-A | DNA-A coated nanoprisms (Figure 3.4 right) were soluble in the water layer only due to 
the hydrophilicity of the DNA coating. HexaD | DNA-A amphiphilic Janus nanoprisms lie at the interface of hydrophobic and hydrophilic solvent, (Figure 3.4 middle), confirming the coexistence of hydrophobic HexaD and hydrophilic DNAA coating on the HexaD | DNA-A Janus nanoprisms.

It is worth noting that although HexaD | DNA-A Janus nanoprisms are dispersible in water in the absence of a chloroform layer, the particles dynamically realign themselves in response to a bulk hydrophilic/hydrophobic interface. One can assume that when Janus nanoprisms are suspended at the water/chloroform interface, the hydrophilic DNA-coated facet is oriented towards the water layer while hydrophobic HexaD layer orients towards chloroform to reduce the total interfacial energy. A similar phenomenon was reported in previous studies of isotropic core amphiphilic particles. ${ }^{194-195}$ Furthermore, Both DNA-A | HexaD, where nanoprisms were coated with DNA-A first and then HexaD, as well as HexaD | nanoprisms, where nanoprisms were coated with HexaD on only one facet while the other facet doesn't have any coating also showed the same type of orientation at the water-chloroform interface. HexaD | DNA-A amphiphilic Janus nanoprisms lie at the interface of the hydrophobic and hydrophilic solvent, (Figure 3.4 middle), confirming the coexistence of hydrophobic HexaD and hydrophilic DNA-A coating within the HexaD | DNA-A Janus nanoprisms. 


\subsubsection{Selective adsorption of Janus nanoprisms on both hydrophilic and hydrophobic surfaces}

The presence of two mutually exclusive molecular coatings on the two major facets of Janus particles was confirmed via the selective deposition of HexaD | HexaD, HexaD | DNA-A , and DNA-A | DNA-A nanoprisms on either hydrophilic or hydrophobic surfaces. To accomplish this, silica glass slides were made hydrophobic or hydrophilic with the treatment of poly-siloxanes and APTES, respectively (See the Experimental for details). Figure 3.5 shows darkfield microscopy images of HexaD | DNA-A Janus nanoprisms along with DNA-A and HexaD single species nanoprisms on the hydrophilic and hydrophobic surfaces. Both SEM images (Figure 3.6) and the characteristic nIR dominance of the scattering spectra confirm the bright spots are due to nanoprisms (Figure 3.6 and

Figure 3.5 insets). The $\lambda_{\max }$ of the scattering spectra are blue shifted compared to the SPR maxima of colloidal coated and uncoated nanoprisms (Figure 3.2A) due to the low refractive index of air/silica glass compared to ethanol and water. ${ }^{196}$ DNAA | DNA-A nanoprisms are hydrophilic and hence adsorbed well on the APTES surface (Figure 3.5A and 3.6), while hydrophobic HexaD | HexaD nanoprisms did not bind to the hydrophilic APTES surface (Figures 3.5C and 3.6). Conversely, no binding between hydrophilic DNA-A | DNA-A nanoprisms and the hydrophobic poly-siloxane surface was observed (Figures 3.5D and 3.6), whereas HexaD | HexaD nanoprisms adsorbed well on the hydrophobic poly-siloxane surface, Figures 3.5F and 3.6. 
Additionally, HexaD-coated nanoprisms were randomly stacked together to form small aggregates as shown in the darkfield and SEM image (Figure 3F and S6) and displayed heterogenous broadening in the scattering spectrum, (Figure 3.5F inset). While HexaD | HexaD nanoprisms and DNA-A | DNA-A nanoprisms were only bound to either the poly-siloxane or APTES surface, amphiphilic Janus nanoprisms exhibited binding interactions with both APTES (Figure 3.5B and 3.6) and polysiloxane surfaces (Figure 3.5E and 3.6), presumably through their hydrophilic and hydrophobic facets, respectively. This confirms the presence of only DNA-A on one facet and HexaD coating on the other facet of Janus nanoprisms. Due to their hydrophilicity, DNA-A coated nanoprisms bind only to the APTES surface while HexaD-coated nanoprisms bind to only the poly-Siloxanes surface because of the hydrophobicity of the HexaD coating. However, amphiphilic Janus nanoprisms bind to both APTES and Poly Siloxanes surface due to their amphiphilicity. Note that while particle concentrations were different for different types (Janus, DNA-A | HexaD coated), the concentration for each type was consistent for the APTES and poly-siloxane surfaces. Also note that after drop casting, Janus particles (in ethanol) spread out evenly on APTES surface, but they form concentrated droplets on polysiloxanes, which contributed to more particle population for any given area on poly siloxanes than on APTES. 


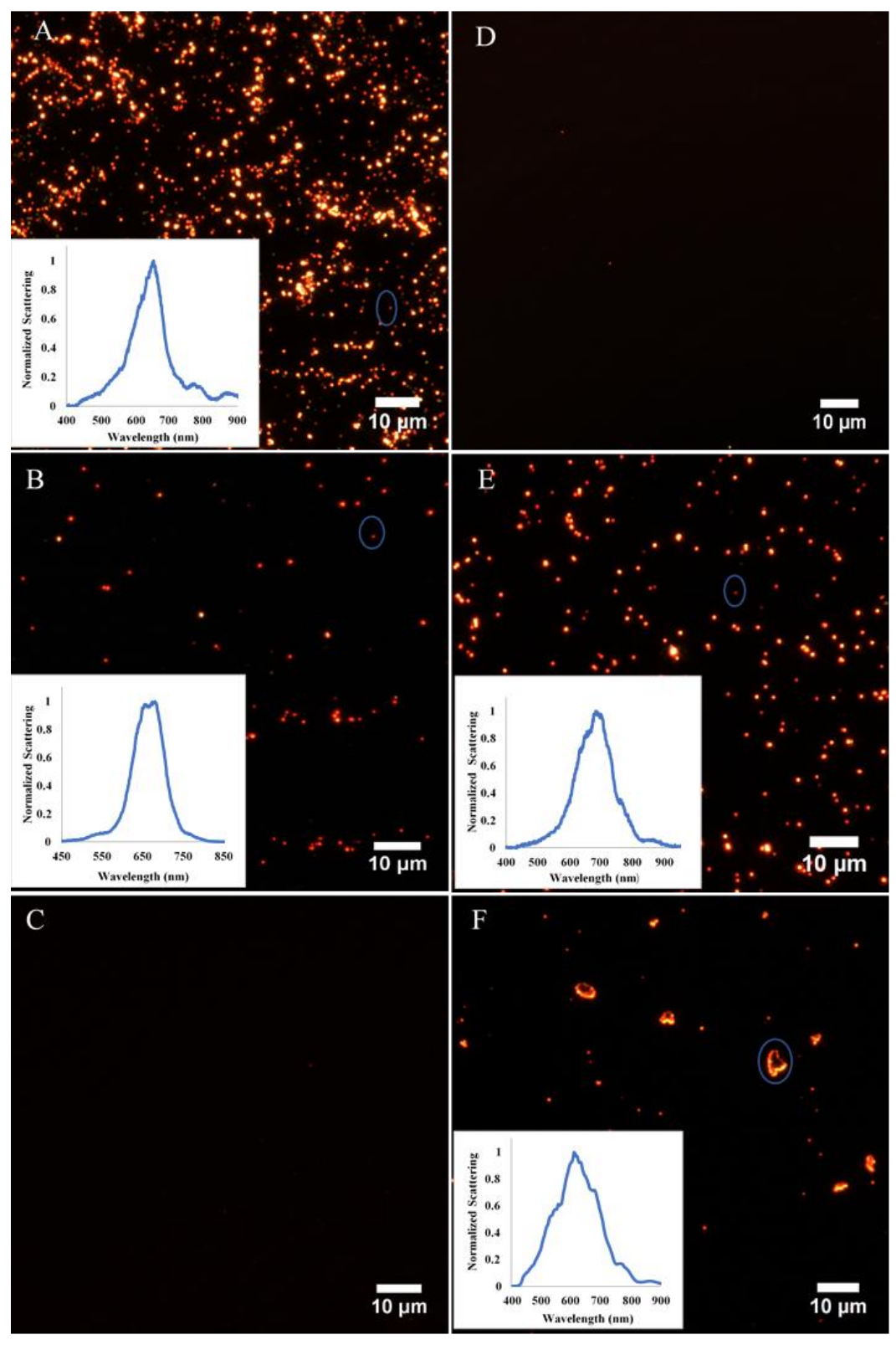

Figure 3.5: Darkfield images of coated nanoprisms on hydrophilic and hydrophobic surface. (A-C) Deposition of DNA-A | DNA-A, HexaD | DNA-A Janus amphiphilic, and HexaD | HexaD nanoprisms on APTES respectively. (D-F) Deposition of DNA-A | DNA-A, HexaD | DNA-A amphiphilic Janus, and HexaD | HexaD nanoprisms on poly-siloxane, respectively. The insets show representative scattering spectra of the circled particles. 


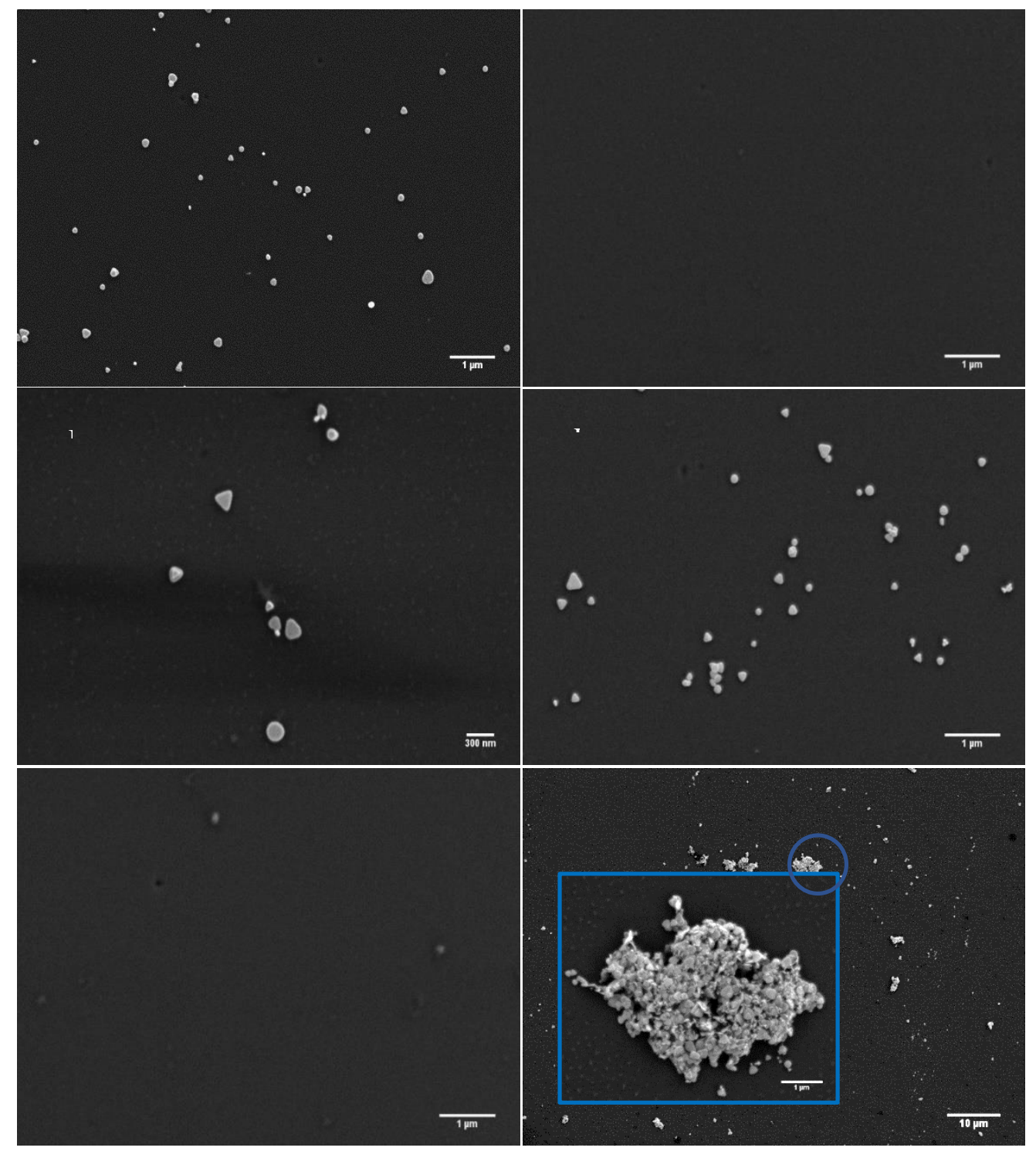

Figure 3.6: Representative SEM images of coated nanoprisms on hydrophilic and hydrophobic surfaces. (A-C) Deposition of DNA-A | DNA-A, HexaD | DNA-A Janus amphiphilic, and HexaD | HexaD nanoprisms on APTES, respectively. (D-F) Deposition of DNA-A | DNA-A, HexaD | DNA-A amphiphilic Janus, and HexaD | HexaD nanoprisms on poly-siloxane, respectively. The inset in F illustrates the formation of aggregates of HexaD-coated nanoprisms. 


\subsubsection{Asymmetric self-assembly behavior of Janus nanoprisms}

To further demonstrate the efficiency of this asymmetric functionalization strategy of anisotropic nanoprisms and their potential application for high throughput nanocluster assembly, Janus PEG | DNA-A nanoprisms were allowed to react with $20 \mathrm{~nm}$ gold nanoparticles coated with DNA-A", which was complementary to DNA-A. A control experiment was also carried out by reacting DNA-A" coated nanospheres with two sets of nanoprisms, where one set was coated with DNA-A only and the other was coated with PEG only. Figure 3.7 shows UV-Vis characterization of binding interactions between DNA-A" coated nanospheres and coated nanoprisms as well as representative SEM images of the various assemblies that resulted from those interactions. The SPR band of PEG coated nanoprisms remains unchanged after mixing with DNA-A"-coated nanospheres (Figure 3.7A), indicating that DNA-A" nanospheres did not bind to PEG-coated nanoprisms as evidenced by SEM analysis (Figure 3.7D\&G).

This is due to the lack of specific molecular interaction and the well-known proclivity of PEG-coated surfaces to resist adsorption events. On the other hand, the SPR band for both Janus and DNA-A-coated nanoprisms red-shifted and broadened after mixing with DNA-A" nanospheres, indicating strong binding affinity and specific molecular interactions between DNA-A" nanospheres, and both Janus and DNA-A-coated nanoprisms (Figure 3.7B and 3.7C). The shifts in the SPR are due to dipole-dipole interactions between the nanoprisms and nanospheres as the binding events bring them into close proximity. Notably, as 

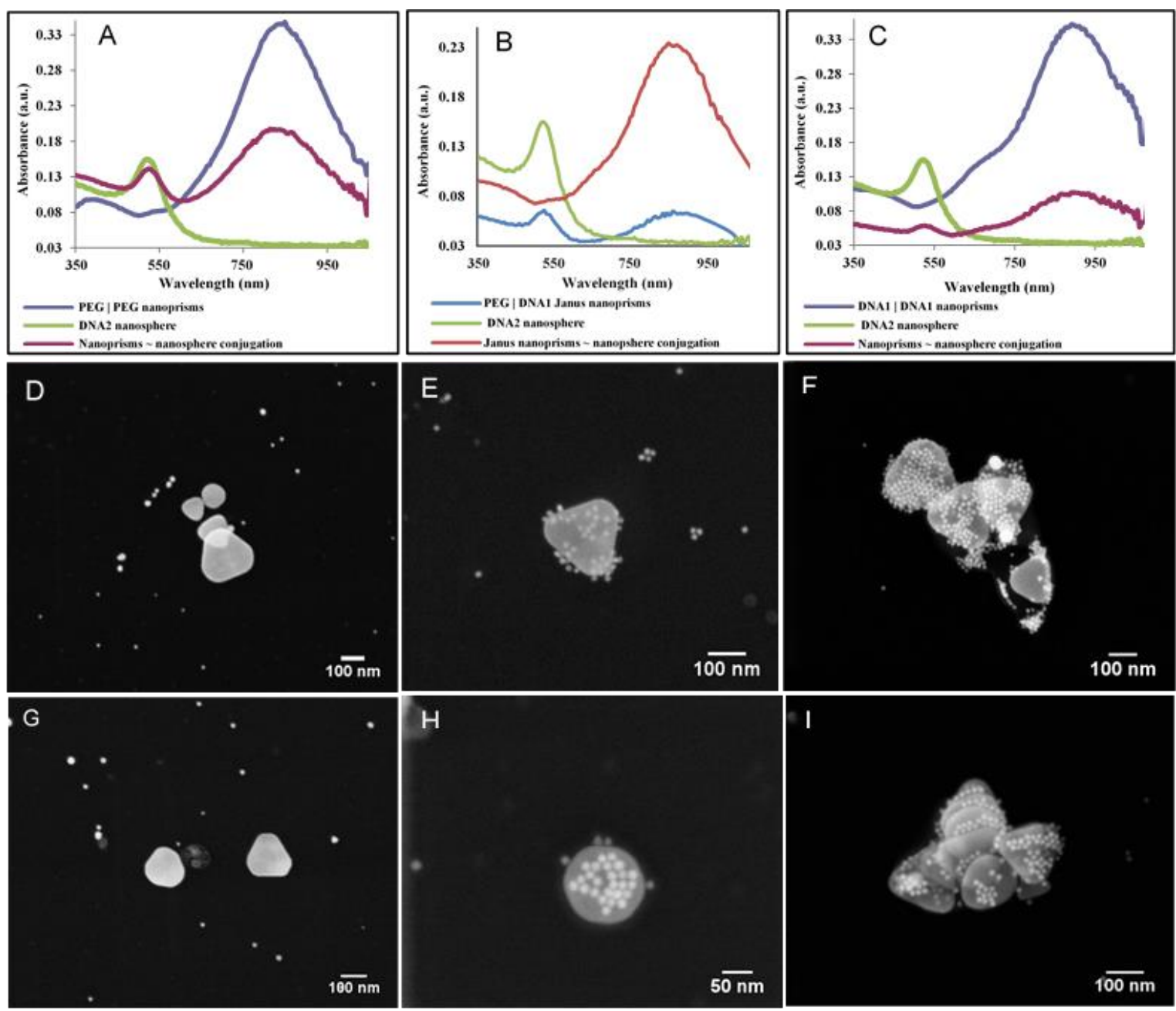

Figure 3.7: UV- Vis characterization of binding interactions between DNA-A" nanospheres and (A) PEG | PEG nanoprisms, (B) Janus PEG | DNA-A nanoprisms, and (C) DNA-A | DNA-A nanoprisms and representative SEM images of binding interactions between DNA-A" coated nanospheres (D\&G) PEG | PEG nanoprisms, (E\&H) Janus PEG | DNA-A nanoprisms, (F\&I) DNA-A | DNA-A nanoprisms.

DNA-A" nanospheres are not asymmetrically functionalized and complementary DNA binding events are possible on both facets of the DNA-A | DNA-A nanoprisms, nanospheres bound to both facets of DNA- -coated nanoprisms form aggregates (Figure 3.7F\&I), while Janus PEG | DNA-A nanoprisms showed 
binding interactions with DNA-A" nanospheres through one facet only (Figure 3.7E\&H ). Since DNA-A" nanospheres doesn't show binding interaction with PEG coated surface (Figure 3.7D\&G), the interaction between Janus particle and DNA-A" spheres could happen through DNA-A containing facet only, indicating successful PEG | DNA asymmetric functionalization. Unlike the interaction of DNA-A-coated nanoprisms and DNA-A" nanospheres, the reaction between Janus nanoprisms and nanopsheres didn't result in aggregation due to the presence of PEG on one facet. This facet selective functionalization of anisotropic nanoprisms could be transferable to other anisotropic particles and hence could provide for powerful and complex assembly schemes for the synthesis of various novel nanostructures such as homo and hetero plasmonic dimers and trimers of anisotropic particles. ${ }^{197}$

\subsection{Conclusions}

In summary, we have demonstrated a facile and versatile approach to controllably coat each major facet of gold nanoprisms with distinct molecular coatings to create Janus nanoprisms. The majority of the previous studies reported on Janus particles focused on creating anisotropy via surface modification of isotropic particles. To the best of our knowledge, this study is the first report of Janus nanoparticles that are synthesized from anisotropic gold nanoprisms. Our Janus nanoparticles are soluble in water, but dynamically orient themselves at the interface of hydrophilic-hydrophobic solvent systems, and exhibit facet selective interactions and asymmetric assembly behavior. The ability to coat one major facet of nanoprisms while keeping the other 
major facet protected could provide a unique platform to fabricate multifunctional Janus particles with directed interactions and complex self-assembly potential. 


\section{CHAPTER 4}

\section{DNA-MEDIATED HIERARCHICAL ORGANIZATION OF GOLD NANOPRISMS INTO 3D SUPERLATTICES FOR SURFACE-ENHANCED RAMAN SCATTERING AND FLUORESCENCE}




\subsection{Introduction}

The assembly of nanoparticles into higher order plasmonic nanostructures with tunable spacing provides a path towards the development of tailorable functional materials with collective properties. ${ }^{198-200}$ In turn, these properties have been utilized for different applications including magnetics, ${ }^{201}$ nanoplasmonics,${ }^{202}$ plasmon enhanced spectroscopies,${ }^{203}$ nanophotonics, ${ }^{204}$ and optoelectronics. ${ }^{205}$ Maximum utility of nanoparticle arrangement towards the realization of novel nanomaterials relies upon the ability to control the orientation and interparticle spacing within the superlattices, as well as crystal symmetry and geometry. ${ }^{206-207}$ Therefore, hierarchical organization of nanoparticle into plasmonic superstructure remains an important research topic in nanoscience.

Common top-down and bottom-up strategies to fabricate plasmonic superlattices includes Electron beam lithography, ${ }^{208-209}$ the Langmuir-Blodgett technique,${ }^{210}$ the droplet evaporation method, ${ }^{211-212}$ interface-based assembly, ${ }^{213}$ and acoustic levitation techniques. ${ }^{214}$ All of these methods are either labor intensive and costly or inefficient in terms of reproducibility and specificity. ${ }^{100}$ DNA programmed assembly have emerged as a powerful route for hierarchical organization of nanoparticles with a high degree of control over the placement of nanoparticles. ${ }^{110,215-218}$ The programmability of the DNA length, nucleobase sequencing specific complementary DNA interaction and simple interaction rules allow fine control in tuning the interparticle spacing, lattice symmetry, and nanoparticle composition. ${ }^{99,115,219}$ These properties make DNA induced assembly of nanoparticles an ideal platform for developing highly ordered 2D and 3D plasmonic superlattices from both spherical and nonspherical nanoparticles. 
The ability of plasmonic nanoparticles to concentrate light into nanoscale volumes and create locally enhanced strong electromagnetic (EM) fields has been exploited for a broad array of applications such as optical antennae, ${ }^{220-221}$ ultrasensitive sensors, ${ }^{164,222-223}$ information processing, ${ }^{224}$ surface-enhanced Raman scattering (SERS), ${ }^{225-227}$ surfaceenhanced fluorescence (SEF), ${ }^{159,228}$ and nonlinear optics. ${ }^{229}$ In particular, anisotropic shaped nanoparticles (rods, prisms) such as those made of Au and Ag have been a subject of intense interest in surface enhanced spectroscopies, for instance SERS ${ }^{227,230}$ and SEF, ${ }^{160,231}$ because of their sharp edges and vertices that greatly confine EM field, and the tunablity of their surface plasmon resonance throughout the visible and near-IR (NIR) regions. ${ }^{27,}{ }^{232-233}$ In addition, assembling such nanoparticles in a predesigned spatial arrangement with gap sizes on the order of few nanometers leads to the formation of so called plasmonic hot spots due to nano gap effects. ${ }^{234-235}$ When a Raman reporter molecule or a fluorescence emitter is placed in those hot spots, strong SERS and SEF enhancement are observed due to strong near field coupling between neighboring nanoparticles that induces enormous EM field enhancement. ${ }^{236-237}$ The strength of EM field enhancement within the nanogap can be modulated by several key factors such as gap distance, particle shape/size, and excitation configuration. ${ }^{161}$ The concept of nanogap effects and the desirability of a high density of hot spots in the excitation laser focal volume has led to development of various $2 \mathrm{D}$ and $3 \mathrm{D}$ nanoparticle super lattices with extensive plasmonic coupling to obtain large-area of hotspots. ${ }^{238-239}$

Although programmable DNA assembly has been used to create well defined 3D lattices of anisotropic nanoparticles (gold nanorods, cubes, rhomboids, dodecahedrons), ${ }^{29,}{ }^{128-129}$ the 
formation of such superlattices of anisotropic gold nanoprisms using DNA has not been reported. In this study, we demonstrated a stepwise hierarchical fabrication of 3D plasmonic superlattices from polydisperse anisotropic gold nanoprisms and their application as SERS and SEF substrates. Using DNA as surface ligand and binding motif, prisms were organized into 1D crystals through preferential face-to-face binding, which maximizes DNA interactions. By subjecting 1D nanoprism crystals to extended annealing below the $\mathrm{T}_{\mathrm{m}}$ of crystals, micrometer scale 3D plasmonic nanoprism superlattices composed of densely packed 1D nanoprisms arrays were fabricated. As discussed in section 1.6, the assembly of DNA grafted nanoparticles leads to various complex plasmonic substrates with applications in SERS, SEF, and nanoplasmonics. Accordingly, in this study, we investigated the plasmonic activity and suitability of 3D plasmonic superlattices as SERS and SEF substrates. SERS analysis of a probe molecule, Methylene blue (MB) and photoluminescence (PL) analysis of a fluorescent dye, Alexa fluor Phalloidin, using 3D plasmonic superlattices revealed excellent SERS and PL enhancement. ${ }^{240}$

\subsection{Results and Discussion}

\subsubsection{Face-to-face assembly of gold nanoprisms}

Gold nanoprisms of size range 100-200 nm were synthesized via the Diasynth method and purified following literature precedents. ${ }^{164-165}$ Purified nanoprisms were densely functionalized with two complementary DNA strands, DNA-A and DNA-A" (see section 2.3.3 for details). DNA induced hierarchical assembly of gold nanoprisms was accomplished in two steps. DNA functionalized nanoprisms were first assembled into 1D nanoprism arrays, which were then crystallized into 3D superstructures in the $2^{\text {nd }}$ step via 
long range DNA-driven interactions of 1D nanoprism stacks induced by a thermal annealing.

Gold nanoprisms are essentially 2D nanoobjects with two extended flat surfaces that are significantly larger than their thickness. As DNA mediated assembly of nanoparticles preferentially occurs through binding events that allow maximum hybridization interactions between complementary DNA linkers anchored on the particles' surface, DNA functionalized prisms are expected to preferentially bind in an associative manner via their large flat surfaces. ${ }^{29}$ When an equimolar amount of two sets of nanoprisms functionalized with complementary DNA strands were mixed in a hybridization buffer and heated to 60$65^{\circ} \mathrm{C}$ ( well above $\mathrm{T}_{\mathrm{m}} \sim 41^{\circ} \mathrm{C}$ of DNA strands), prisms assembled in a face-to-face manner leading to the formation of $1 \mathrm{D}$ crystals with lengths varying from $500 \mathrm{~nm}$ to $1.2 \mu \mathrm{m}$ and consisting of 20-40 monomer units, as shown in Figure 4.1. The non-uniformity and size variation of the $1 \mathrm{D}$ nanoprisms crystals can be attributed to the size disparity of the individual building blocks. Prior theoretical and experimental works showed that polydispersity in colloid suspension suppresses the nucleation growth and leads to the formation of different crystallites. ${ }^{241-242}$

The melting temperature $\left(\mathrm{T}_{\mathrm{m}}\right)$ of $1 \mathrm{D}$ nanoprism stacks was determined by monitoring the change in absorbance at the SPR $\lambda_{\max }$ of the nanoprisms as a function of temperature. A control experiment was also carried out by similarly annealing a solution containing uncoated nanoprisms. The melting transition observed for 1D nanoprism stacks, as shown in Figure 4.1D, and the absence of such a profile for the control experiment (Figure 4.2A) as well as the lack of nanoprisms ordering for the control experiment (as shown in the 

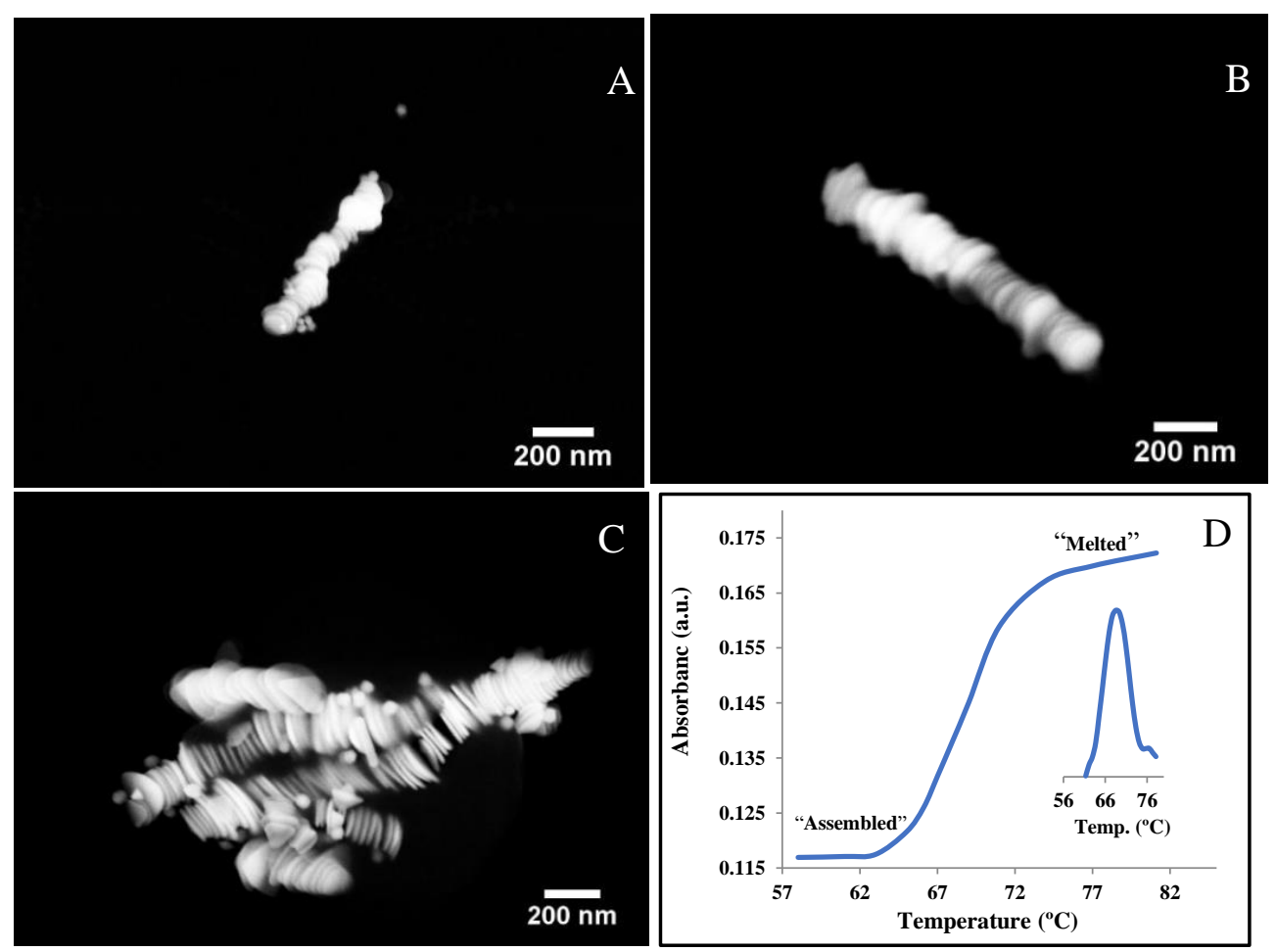

Figure 4.1: DNA mediated Face-to-face assembly of gold nanoprisms. A, B, C) Representative SEM images of DNA mediated face-to-face assembly of nanoprisms into 1D stacks, D) Melting profile of 1D nanoprisms crystals, monitored at the SPR $\lambda_{\max }$ of nanoprisms. Inset shows the first derivative of the melting curve.

SEM image (Figure 4.2B)) indicate that nanoprisms were densely functionalized with DNA and the melting transition occurred due to the de-hybridization of complementary DNA strands that connected nanoprisms in $1 \mathrm{D}$ stacks. The $\mathrm{T}_{\mathrm{m}}$ of $1 \mathrm{D}$ nanoprism stacks was found to be $68.5^{\circ} \mathrm{C}$, determined by taking the first derivative of the melting curve and finding the full width of the peak at half maximum $\left(\mathrm{FWHM}=7.2^{\circ} \mathrm{C}\right)$. The melting transition occurred over a relatively broad range, which could be attributed to the polydispersity of 1D nanoprism stacks. 

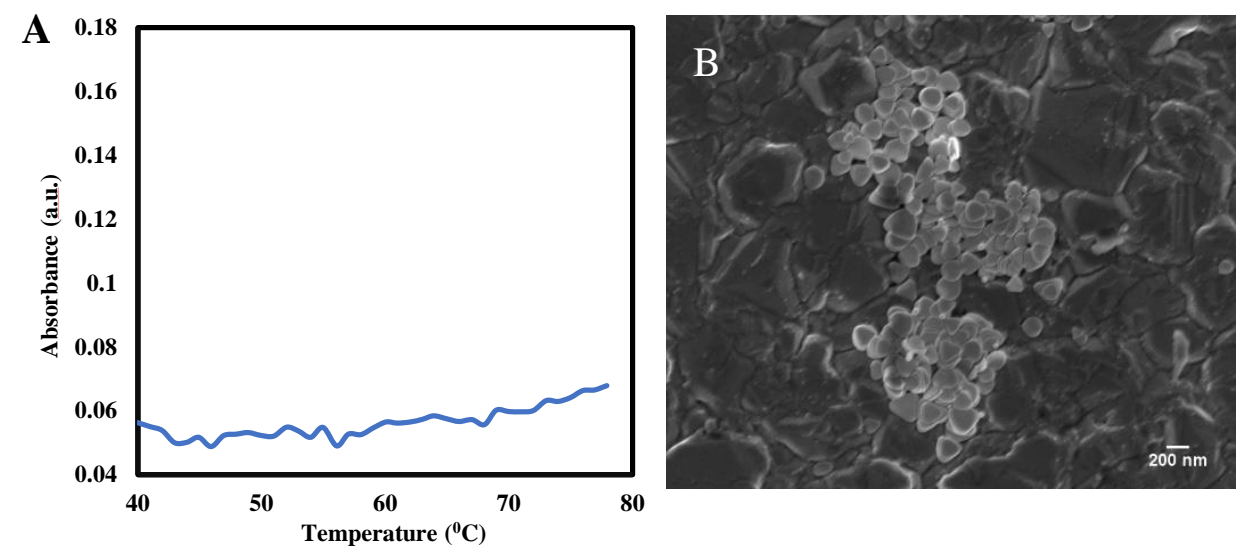

Figure 4.2: A) Melting profile of uncoated nanoprisms in DNA hybridization buffer, and B) SEM image of uncoated nanoprisms after mixing with DNA hybridization buffer.

\subsubsection{Long range ordering of nanoprisms into 3D superlattices}

To achieve the long-range ordering and assembly of 1D nanoprism stacks into 3D superlattices, we adopted the near $\mathrm{T}_{\mathrm{m}}$ annealing nanoparticle crystallization approach. Thermal annealing is often employed for long range ordering and crystallization processes in atomic and nanoscale systems. ${ }^{243-244}$ Thermal annealing below the $T_{m}$ reduces the complimentary DNA-mediated attraction energy and increase the frequency the dehybridization events, which promotes greater particle rearrangement and long range ordering without aggregate dissociation. ${ }^{114,245}$ Prior to thermal annealing below $\mathrm{T}_{\mathrm{m}}$, the dispersion of 1D nanoprism stacks was spun down and resuspended in $250-300 \mu \mathrm{L}$ phosphate buffer in order to concentrate 1D nanoprism stacks. The organization of 1D 
nanoprism stacks and crystal growth was not observed for dilute dispersions of stacks even with extended annealing at elevated temperatures.

Concentrated dispersions of $1 \mathrm{D}$ nanoprism stacks were gradually heated at $65^{\circ} \mathrm{C}, 66^{\circ} \mathrm{C}$ and $67.5^{\circ} \mathrm{C}$ (below $\mathrm{T}_{\mathrm{m}} \sim 68.5^{\circ} \mathrm{C}$ ) for a period of $1-3$ hours (see section 2.3 .13 for details).

Dark field microscopy was used to characterize the long-range ordering and crystallization of nanoprisms as thermal treatment progressed. As illustrated in dark field microscopy images, Figure 4.3, when the thermal annealing was applied, 1D nanoprism stacks started to hierarchically assemble into $3 \mathrm{D}$ superlattices. As the temperature increased from $55^{\circ} \mathrm{C}$ to just below $\mathrm{T}_{\mathrm{m}}$ of $1 \mathrm{D}$ nanoprism stacks, the crystal grain size continued to grow larger.

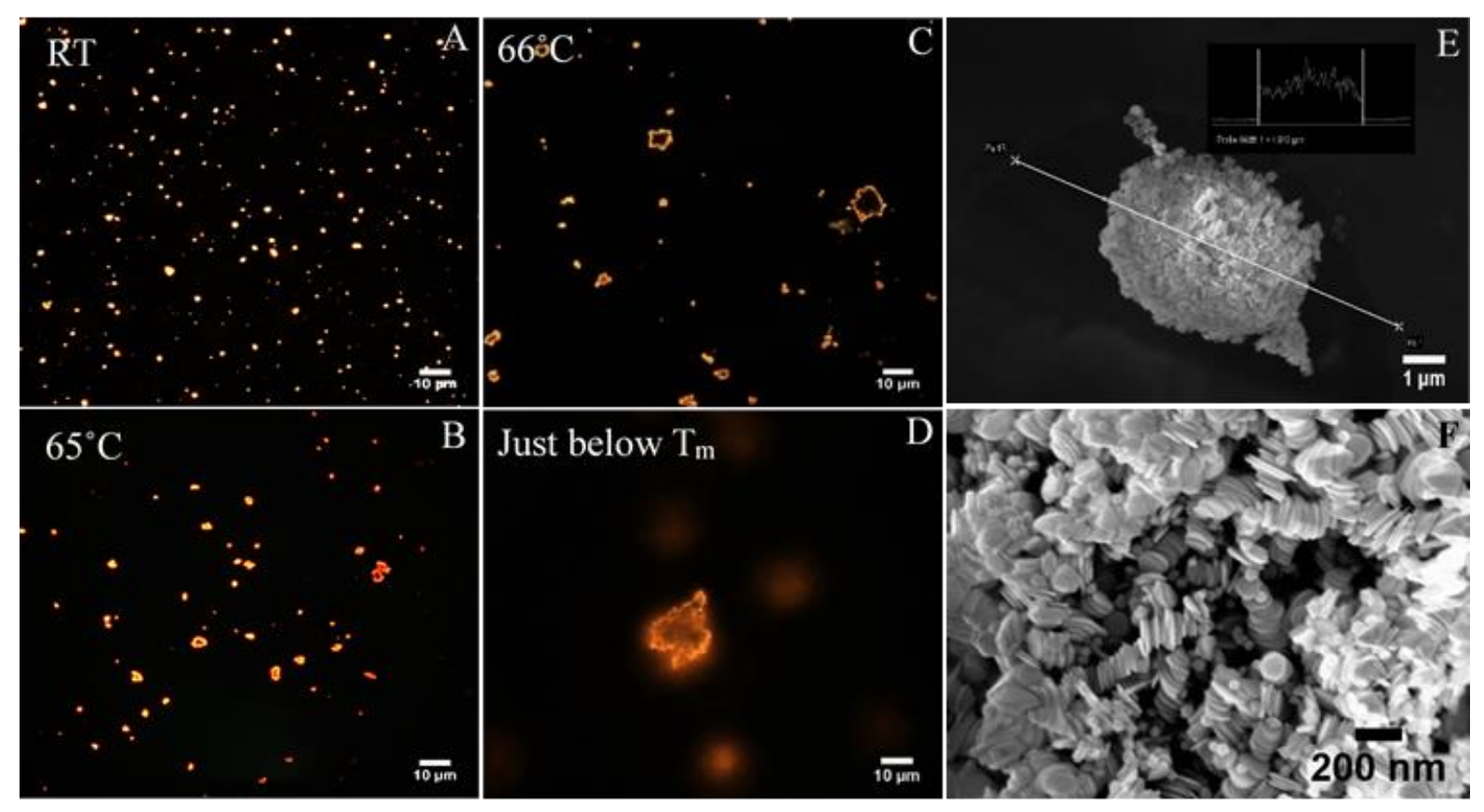

Figure 4.3: DNA mediated 3-D hierarchical organization of gold nanoprisms. Dark field microscopy images of long-range ordering of 1D nanoprism stacks at A) Room temperature, B) $65^{\circ} \mathrm{C}$, C) $66^{\circ} \mathrm{C}$ and D) Just below $\mathrm{T}_{\mathrm{m}} \sim 68.5^{\circ} \mathrm{C}$. E, F) Representative low and high magnification SEM images of 3D nanoprisms superlattice formed below $\mathrm{T}_{\mathrm{m}}$, respectively. 
Figures 4.3 E shows representative SEM images of nanoprism superlattices formed below $\mathrm{T}_{\mathrm{m}}$, which are highly polycrystalline with crystal defects and exhibit a size distribution from 5-15 $\mu \mathrm{m}$ in diameter. In contrast, previous studies on DNA driven assembly of gold nanoprisms afforded only simple 1D face-to-face stacking. ${ }^{29,}{ }^{246}$ High magnification SEM images (Figure 4.3F) allow closer inspection of the 3D polycrystalline nanoprism superlattices, revealing both lateral and perpendicular orientations of 1D nanoprism stacks and the presence of crystal defects within the superlattices. The polycrystallinity of the nanoprism superlattices differs from the well-defined hexagonal or honeycomb superlattices achieved by salt mediated crystallization of nanoprisms, ${ }^{247-248}$ and those predicted by theoretical study on the phase behavior of polyhedral particles. ${ }^{249}$ The lack of well-defined hexagonal or honeycomb superlattices of nanoprisms could be due to high polydispersity (around 30-40\%) of the nanoprisms used in this study. Mirkin and coworkers showed that having particles (spheres) with polydispersity less than $10 \%$ is crucial to create well defined DNA-induced programable crystalline structures. ${ }^{114}$

\subsubsection{Surface enhanced Raman scattering (SERS) of 3D nanoprism superlattices}

Close-packed colloidal nanoparticle superlattices give rise to enhanced plasmonic activity. We assessed the plasmonic activity of the 3D nanoprism superlattices for applications in surface enhanced spectroscopies such as surface enhanced Raman scattering (SERS) and surface enhanced florescence (SEF). The SERS enhancement performance of 3D nanoprism superlattices was studied by the SERS analysis of methylene blue (MB) as a model compound. MB is chosen as the model compound for SERS analysis because of its well-known characteristic Raman bands. Prior to Raman 
analysis, nanoprism superlattice substrates were deposited on a glass slide. The hierarchical organization of the nanoprisms remains unchanged after drop casting on glass substrates followed by air-drying as shown in the SEM image, Figure 4.4A. All Raman analyses were carried out on clean silica glass slides under the same experimental conditions, using an excitation wavelength of $633 \mathrm{~nm}$. Figure 4.4B shows the Raman spectra of a bare glass substrate, bare nanoprism superlattices, and a $10^{-3} \mathrm{M}$ aqueous solution of MB on either a glass substrate or 3D nanoprism superlattices. The Raman spectrum recorded for $10^{-3} \mathrm{M} \mathrm{MB}$ on nanoprism superlattices reveals strong Raman peaks with good signal to noise ratio and characteristics peak positions of MB consistent with previous reports. ${ }^{250-252}$ Some of the most prominent bands in the SERS spectra of MB are identified at $1621 \mathrm{~cm}^{-1}$ for $(\mathrm{C}-\mathrm{C})$ ring stretching, $1394 \mathrm{~cm}^{-1}$ for $(\mathrm{C}-\mathrm{N})$ symmetrical stretching, $1298 \mathrm{~cm}^{-1}$ for $(\mathrm{C}-\mathrm{H})$ in-plane ring deformation, $1154 \mathrm{~cm}^{-1}$ for $(\mathrm{C}-\mathrm{H})$ in-plane bending, and $449 \mathrm{~cm}^{-1}$ for $(\mathrm{C}-\mathrm{N}-\mathrm{C})$ skeletal deformation mode. These peaks were not observed in the Raman spectra of the nanoprism superlattices and glass substrates without MB, indicating that SERS signals can be assigned to the probe molecule. While Raman analysis of $10^{-3} \mathrm{M} \mathrm{MB}$ on $3 \mathrm{D}$ nanoprism superlattices reveals a highly structured spectrum with well-defined characteristic peaks of MB, only two peaks with weak intensities were observed at $1621 \mathrm{~cm}^{-1}$ and at $450 \mathrm{~cm}^{-1}$ for the same concentration of MB using glass substrates, indicating that the 3D nanoprism substrates are highly SERS active. 

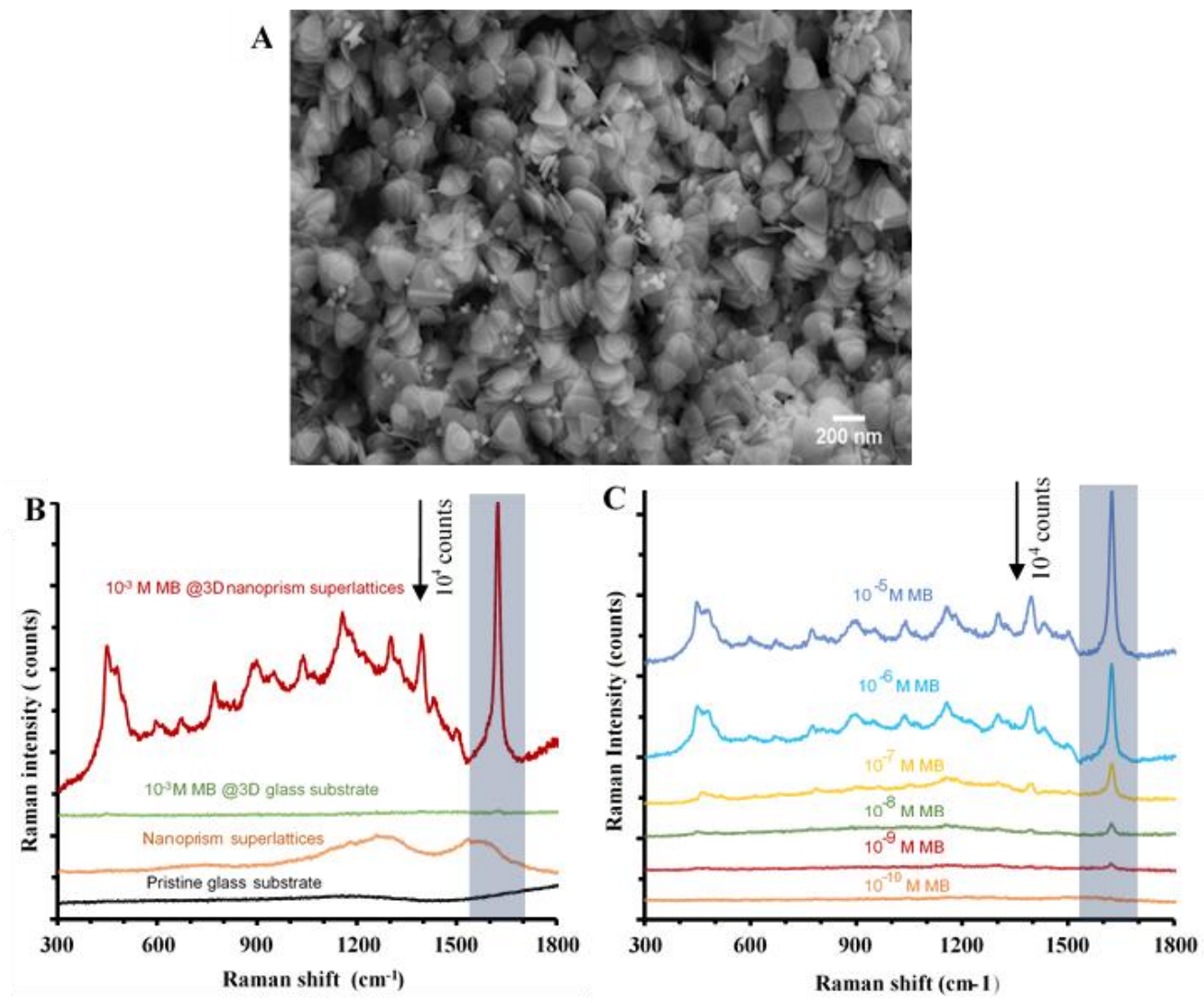

Figure 4.4: A) Representative SEM image of 3-D hierarchical organization of gold nanoprosms on a glass slide. B) Raman spectrum of a pristine glass substrate (black) and 3D nanoprism superlattice (orange), and SERS spectra of $10^{-3} \mathrm{MB}$ adsorbed on glass substrate (green) and 3D nanoprism superlattices (dark red), and C) SERS spectra of MB on 3D nanoprism superlattices at different concentrations.

The strong SERS enhancement for the 3D substrates is attributed to the presence of 3D close-packed organization of nanoprisms columnar arrays, which leads to the formation of a large ensemble of plasmonic hot spots due to enhanced plasmonic coupling and field enhancement. The significant enhancement of Raman intensity for the band at $1621 \mathrm{~cm}^{-1}$ indicates a favorable orientation and adsorption of probe molecules to the nanoprisms' surface. ${ }^{253}$ 
To determine the molecular detection limit of 3D nanoprism superlattices, SERS spectra for a series of concentrations of MB were collected. Figure 4.4C shows the SERS analysis of $\mathrm{MB}$ at different concentrations ranging from $10^{-5}$ to $10^{-10} \mathrm{M}$. The intensity of SERS peaks for MB gradually dropped as the concentration of MB decreased from $10^{-5}$ to $10^{-10} \mathrm{M}$. The SERS spectrum shows a weak peak at $1621 \mathrm{~cm}^{-1}$ for a very low MB concentration of $10^{-10} \mathrm{M}$ (Figure 4.5), indicating that the 3D nanoprism substrate is highly sensitive.

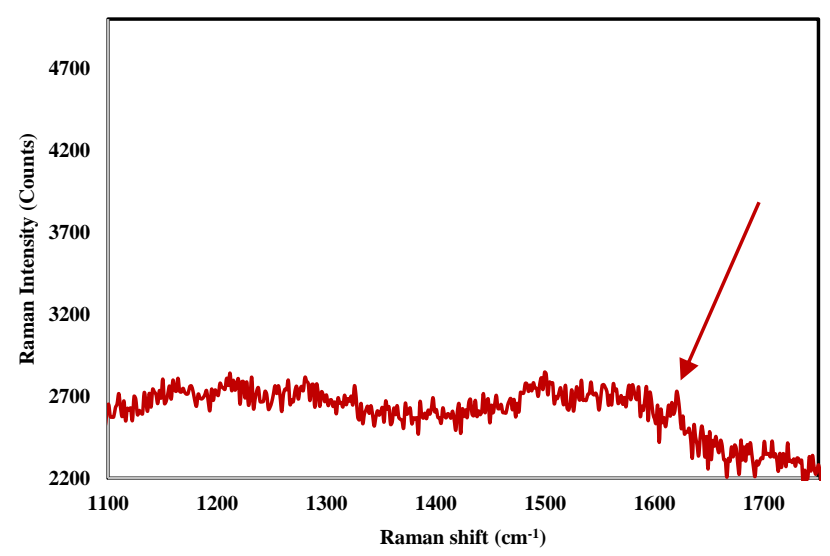

Figure 4.5: SERS spectra of $10^{-10} \mathrm{M} M B$ on $3 \mathrm{D}$ nanoprism superlattices

The SERS enhancement factor (EF) of the 3D nanoprism superlattices was calculated from the SERS intensity of the prominent band at $1621 \mathrm{~cm}^{-1}\left(10^{-9} \mathrm{M} \mathrm{MB}\right)$ and Raman intensity of the corresponding band $\left(10^{-3} \mathrm{M} \mathrm{MB}\right)$, considering the bare glass substrate as the reference (see Experimental details for details). 
The SERS enhancement of MB on the 3D nanoprism superlattices was assessed by calculating the enhancement factor (EF) using the following equation. ${ }^{233}$

$$
E F=\frac{I_{S E R S}}{I_{\text {Raman }}} \frac{C_{\text {Raman }}}{C_{S E R S}}
$$

where ISERS and $I_{\text {Raman }}$ refer to the peak intensity at $1621 \mathrm{~cm}^{-1}$ in the SERS spectrum of $10^{-7}$ $\mathrm{M} M B$ on the 3D SERS substrate and in the Raman spectrum of $10^{-3} \mathrm{M} M B$ on the reference glass substrate, respectively. $\mathrm{C}_{\text {SERS }}$ and $\mathrm{C}_{\text {Raman }}$ refers to the concentration of MB in the SERS and Raman experiment, respectively.

The SERS signal intensity at $1621 \mathrm{~cm}^{-1}$ for $10^{-9} \mathrm{M} \mathrm{MB}$, ISERS $=67.7 \mathrm{cps}$, and normal Raman signal intensity at $1621 \mathrm{~cm}^{-1}$ for $10^{-3} \mathrm{M} \mathrm{MB}, \mathrm{I}_{\text {Raman }}=22.3 \mathrm{cps}$.

$$
E F=\frac{67.7}{22.3} \frac{10^{-3}}{10^{-9}}=2.91 \times 10^{6}
$$

The EF value is estimated to be $2.91 \times 10^{6}$. These results obtained using 3D nanoprism superlattices are comparable to or better than that of other nanoparticle-based plasmonic SERS substrates for the SERS analysis of MB. ${ }^{251-252,254-256}$

In order to assess the reliability and reproducibility of 3D nanoprism superlattices as SERS substrates, we adopted a statistical approach to quantify the variation in the SERS response between different samples as well as between different spots on a particular sample. To determine sample to sample variation, SERS measurements were recorded for 5 different samples and for each sample, the signal intensity at $1621 \mathrm{~cm}^{-1}$ was measured from at least 6 different spots. Figure 4.6A shows sample to sample variation in the 
average SERS counts at the $1621 \mathrm{~cm}^{-1}$ across 5 different samples. The average signal intensity across the 5 samples was 19194 counts with a coefficient of variation (CV) of only $5.6 \%$, indicating excellent reproducibility of the 3D nanoprism superlattices as SERS substrates.

The spot-to-spot variation was determined by measuring SERS intensity at $1621 \mathrm{~cm}^{-1}$ from more than 25 spots of a sample. Figure 4.6B shows spot to spot variation in the SERS response at $1621 \mathrm{~cm}^{-1}$ and the average SERS intensity for the 3D nanoprism superlattices was 19619 counts with a CV of $28 \%$.
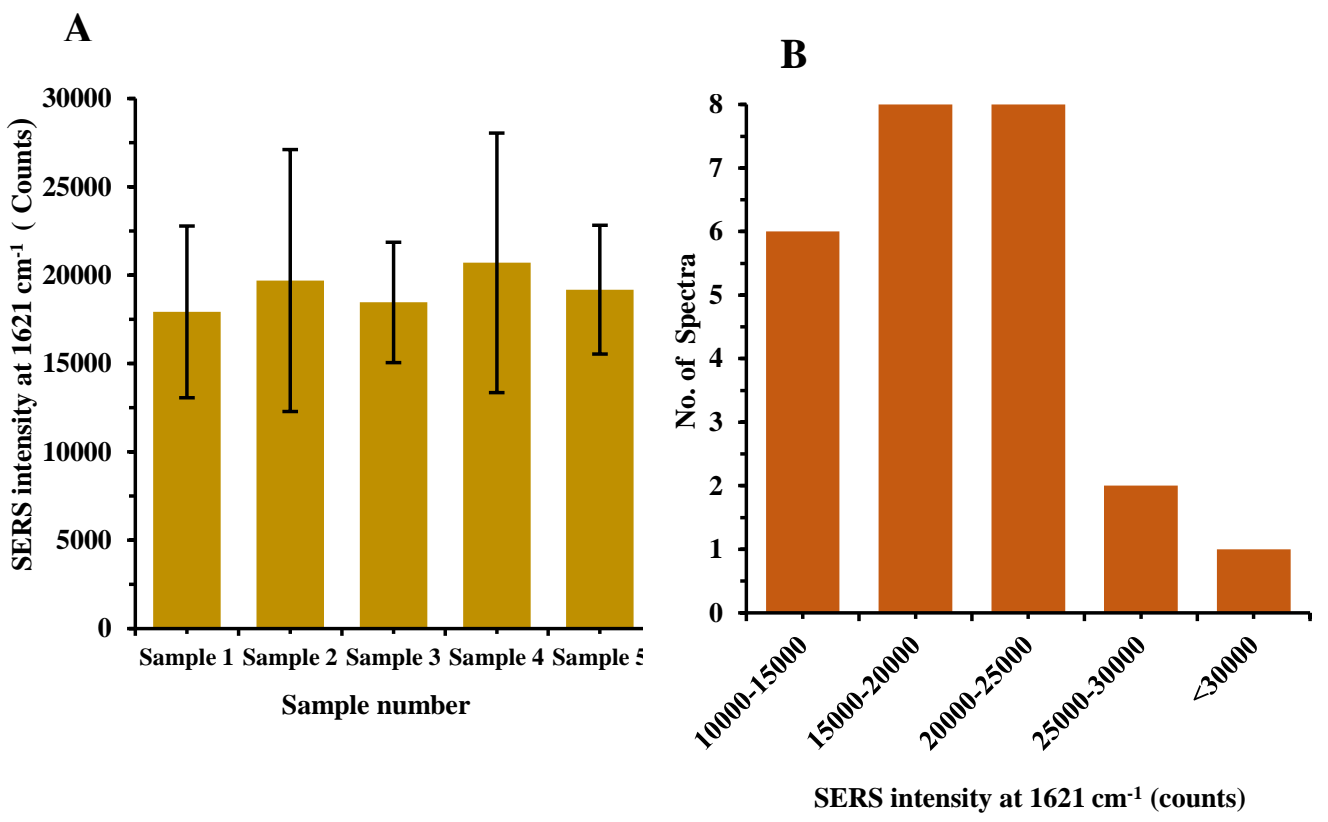

Figure 4.6: A) Sample-to-sample SERS intensity variation at $1621 \mathrm{~cm}^{-1}$ recorded from 5 different substrates. Error bars represent standard deviations of SERS intensity at $1621 \mathrm{~cm}^{-1}$ from at least 6 spots, B) Spot-to-spot variation in the SERS intensity at $1621 \mathrm{~cm}^{-1}$ for the 3D nanoprism superlattices. All measurements were carried out at $\mathrm{MB}$ concentration of $10^{-4} \mathrm{M}$. 
The large spot-to-spot variation in the SERS signal can be attributed to dispersity in the crystal size, which results in varying degrees of electromagnetic field enhancement and plasmonic coupling. To investigate the crystal size effect on the SERS enhancement, we recorded SERS spectra of 10 different spots or 3D nanoprism superlattices of different sizes. As shown in Figure 4.7, the larger the crystal size, the stronger the SERS enhancement, which is consistent with a previous study on the SERS enhancement of nanoparticle-based metamolecules. ${ }^{163}$

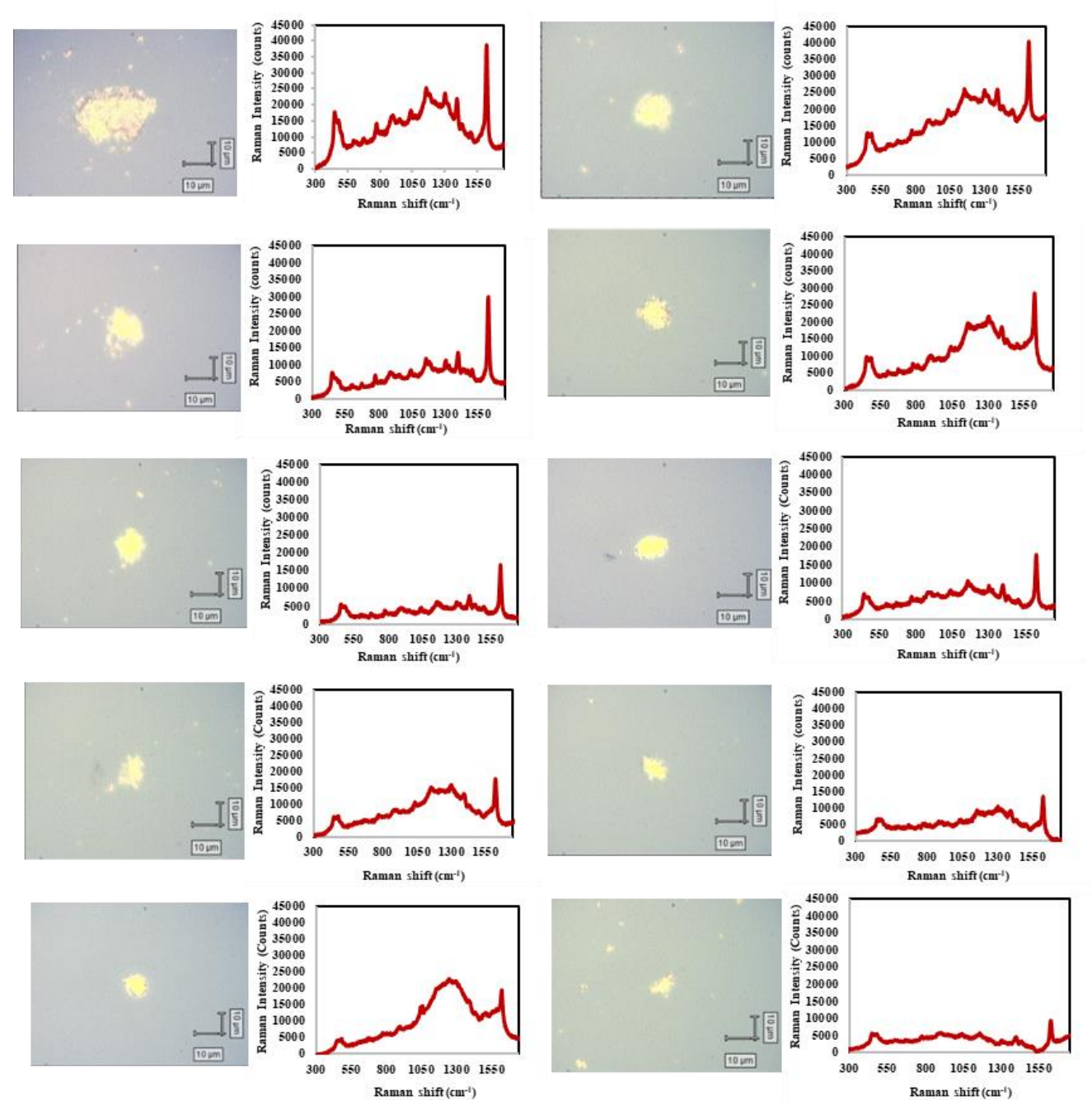

Figure 4.7: Bright field microscopy images and corresponding SERS spectra $\left(10^{-4} \mathrm{M} \mathrm{MB}\right)$ of 10 different spots. 


\subsubsection{Surface enhanced fluorescence spectroscopy of 3D superlattice}

Plasmonic metal nanoparticles have the ability to confine surface plasmon resonance

in a small volume, leading to remarkable enhancement of local electromagnetic fields, and when placed near emitters or fluorophores such as fluorescent dyes or quantum dots, can increase emission rate and quantum yield. ${ }^{160}$

We examined the surface-enhanced fluorescence enhancement capability of 3D nanoprism superlattices by measuring photoluminescence (PL) of a fluorescent dye, Alexa flour 647 phalloidin, with and without nanoprism superlattices. Alexa flour phalloidin 647 is widely used to visualize and quantitate F-actin in tissue sections, cell cultures, or cellfree preparations. PL measurements were collected by illuminating the sample with a 633 $\mathrm{nm}$ HeNe laser. The PL enhancement factor was calculated by normalizing the fluorescence of the dye on 3D nanoprism substrates to that of the control (without 3D nanoprism superlattice). Figure 4.8 shows the PL spectra of the dye on 3D nanoprism superlattices and control glass substrate (see Experimental details for details). When the dye was excited on 3D nanoprism superlattices, significant enhancement (maximum of 5.5 folds) was observed, which could be attributed to strong interaction between dipole moments of the excited dye molecules and surface plasmons generated on the 3D gold nanoprism substrates. ${ }^{257}$ 


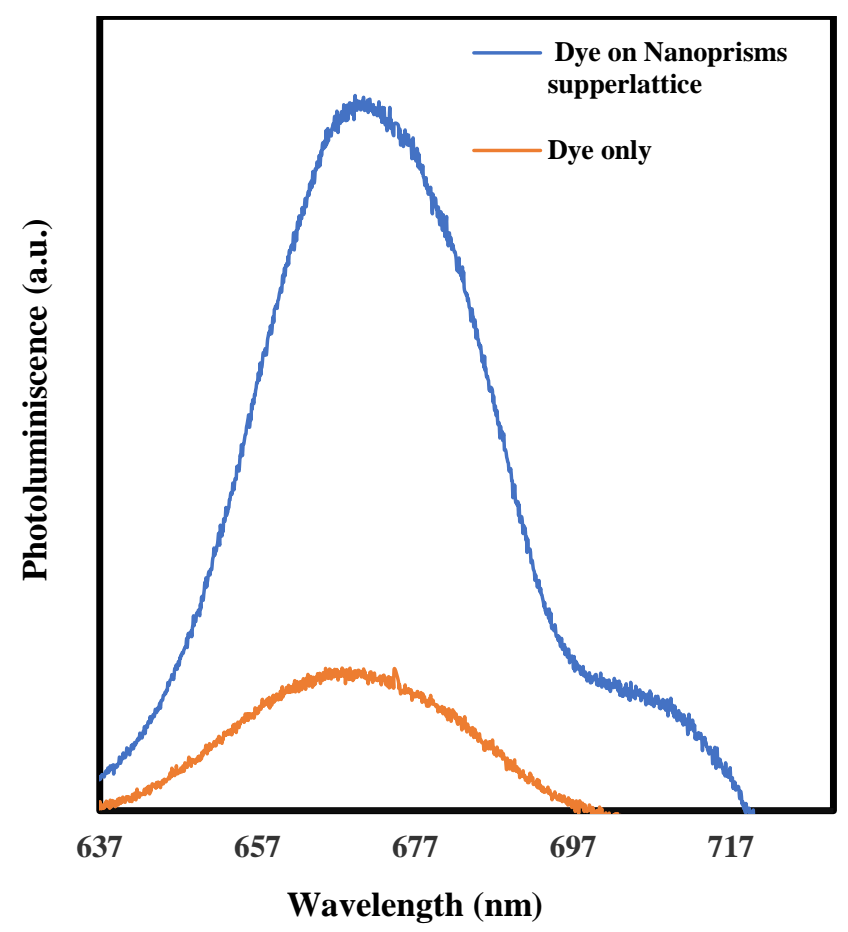

Figure 4.8: PL spectra of Alexa Fluor Phalloidin 647 with (blue) and without 3D nanoprisms superlattices (orange) on a glass substrate at laser wavelength of $633 \mathrm{~nm}$.

\subsection{Conclusion}

In summary, we have demonstrated a DNA-mediated hierarchical assembly of polydisperse gold nanoprisms into 3D superlattices. DNA functionalized nanoprisms were first assembled into 1D columnar stacks of nanoprisms. Thermal annealing below $\mathrm{T}_{\mathrm{m}}$ of $1 \mathrm{D}$ nanoprism stacks facilitated long range interactions between 1D nanoprism stacks and therefore assembly into 3D superlattices with sizes ranging from 5-15 $\mu \mathrm{m}$ in diameter. The large number of closely arranged nanoprism arrays in the $3 \mathrm{D}$ crystal led to the formation of an ensemble of plasmonic hot spots, which gave rise to significant enhancement in SERS and 
SEF. SERS analysis of a probe molecule, methylene blue, using a 3D nanoprism substrate showed significant enhancement $\left(<10^{6}\right)$, high detection sensitivity (as low as $10^{-10} \mathrm{M}$ ) and excellent reproducibility. Moreover, PL analysis of a fluorescent dye, Alexa Fluor Phalloidin 647 using the 3D substrate showed a maximum of 5.5-fold increase in PL intensity of the dye. These findings demonstrate that 3D superlattices of anisotropic nanoparticles could find applications in diverse fields such as photonics, chemical sensing, lasing, and nano-plasmonic waveguide. 


\section{CHAPTER 5}

\section{DNA-INDUCED ASSEMBLY OF GOLD NANOPRISMS AND POLYSTYRENE BEADS INTO 3D PLASMONIC SERS SUBSTRATES}




\subsection{Introduction}

The application of micron sized spherical polymer beads as a carrier medium or support for immobilizing metal nanoparticles has proven to be a useful tool for the fabrications of large areas of SERS substrates. ${ }^{142,258-259}$ The plasmonic coupling between neighboring nanoparticles on the beads' surface in their assembly create plasmonic hot spots, which leads to significant Raman enhancement. ${ }^{260}$ Moreover, the presence of a large number of hot spots at the focal volume of the Raman excitation laser afforded by large surface area of polymer beads are useful and desirable for SERS- based sensing platform. ${ }^{261}$ Various methods have been proposed to immobilize a dense coverage of metal nanoparticles on the beads. ${ }^{132-133,137,141,262-263}$ Lee and coworkers reported a solvent controlled swelling and hetero coagulation method to synthesize highly light scattering metal nanoparticle-coated polystyrene (PS) beads for plasmonic and SERS applications. ${ }^{130-131}$ The immobilization of gold nanostars on PS beads to construct multifunctional SERS probes was also reported. ${ }^{264}$ Furthermore, the high density loading of gold nanospheres using programmable DNA interaction to construct 3D SERS substrate has also been reported. ${ }^{141}$ Several other methods such as covalent coupling, in-situ polymerization, ${ }^{265}$ and phase inversion precipitation methods have been explored for the fabrication of polymer-nanoparticle composite SERS substrates. ${ }^{258,262}$

Anisotropic nanomaterials that exhibit unique shape-dependent plasmonic properties are particularly attractive for SERS based metamaterials. ${ }^{266}$ Anisotropic nanoparticles possess sharp tip and edges that can confine and enhance electric field 
several order of magnitude higher than incident light, leading to formation of hot spots for SERS enhancement. ${ }^{267}$ Moreover, the tunability of plasmon resonance of these anisotropic shape particles over a wide spectral region allows flexibility in designing SERS based sensor. ${ }^{268-269}$ The organization of anisotropic particle with controlled orientation and interparticle distance leads to strong -near-field plasmonic coupling, which provides additional hot spot for SERS enhancement. ${ }^{270}$ For instance, the assembly of nanoprism bowtie configurations with a gap size of only $5 \mathrm{~nm}$ was accomplished by using DNA origami techniques. ${ }^{162}$ The tip-to-tip field coupling between nanoprisms produces strong electromagnetic field enhancement, which led to a mean SERS enhancement factor of about $2.6 \times 10^{9}$ and an electromagnetic field enhancement of about $2.3 \times 10^{3}$. Additionally, the assembly of gold nanorods has been extensively studied for SERS- based detection. Various 3D assembly of gold nanorods have been reported for sensitive detection food contaminant, ${ }^{271}$ pesticides, ${ }^{272}$ and infectious agents ${ }^{273}$. Although there have been numerous reports on the 3D assembly of gold nanorods, the organization of nanoprisms in tip-to-tip, edge-to-edge and tip-toedge orientation over a large surface area and 3D domain has not been realized.

Most previous reports on core shell polymer nanoparticle composite systems focused on loading gold or silver nanospheres on PS beads. ${ }^{130,}$ 141-142 In addition, while several of these methods were successful in increasing particle density on the beads' surface and creating plasmonic hot spots, beads remained isolated from one another, which limited the number of hot spots at the focal volume during SERS analysis. Here we report a DNA-based assembly of anisotropic gold nanoprisms and PS beads into a 
large ensemble of nanoprism-coated PS beads for fabricating a large area of homogenous SERS substrate. Micron sized PS beads were first immobilized with a dense monolayer of nanoprisms to construct PS beads/nanoprism conjugates through DNA hybridization. The initially formed PS beads/nanoprism conjugates were subjected to heating below the melting transition point of the conjugates, followed by slow cooling, which induced the assembly of beads into larger PS beads/nanoprism aggregates comprising 20-50 PS beads that are densely coated nanoprisms. The close packed arrangement of nanoprisms on PS beads' surface generated multiple hot spots in each bead. Additionally, assembling these nanoprism-loaded beads into a larger stack led to the formation a large areas of 3D SERS substrate with a larger number of plasmonic hot spots. SERS analysis of a probe molecule, methylene blue, using the PS beads/nanoprism 3D SERS substrate demonstrated an excellent EF $\left(<10^{5}\right)$ and a detection limit of $10^{-10} \mathrm{M} \cdot{ }^{274}$

\subsection{Results and Discussion}

\subsubsection{Conjugation of nanoprisms to carboxylate-modified PS beads}

Conjugation of nanoprisms to carboxylate-modified PS beads was carried out in two steps (Scheme 5.1). In the first step, carboxylate-modified PS beads were conjugated with an amine-modified thymine rich ss-DNA (DNA-A') via EDC/sulfo-NHS amide bond coupling chemistry (see section 2.3.14 for details) Due to repulsive nature of negatively charged DNA linkers on the PS beads, DNA$\mathbf{A}^{\prime}$ functionalized PS beads were well dispersed in 0.01 M PBS, as shown by fluorescence microscopy image (Figure 5.1). 
Scheme 5.1: Schematic representation of DNA induced loading of gold nanoprisms onto PS beads.
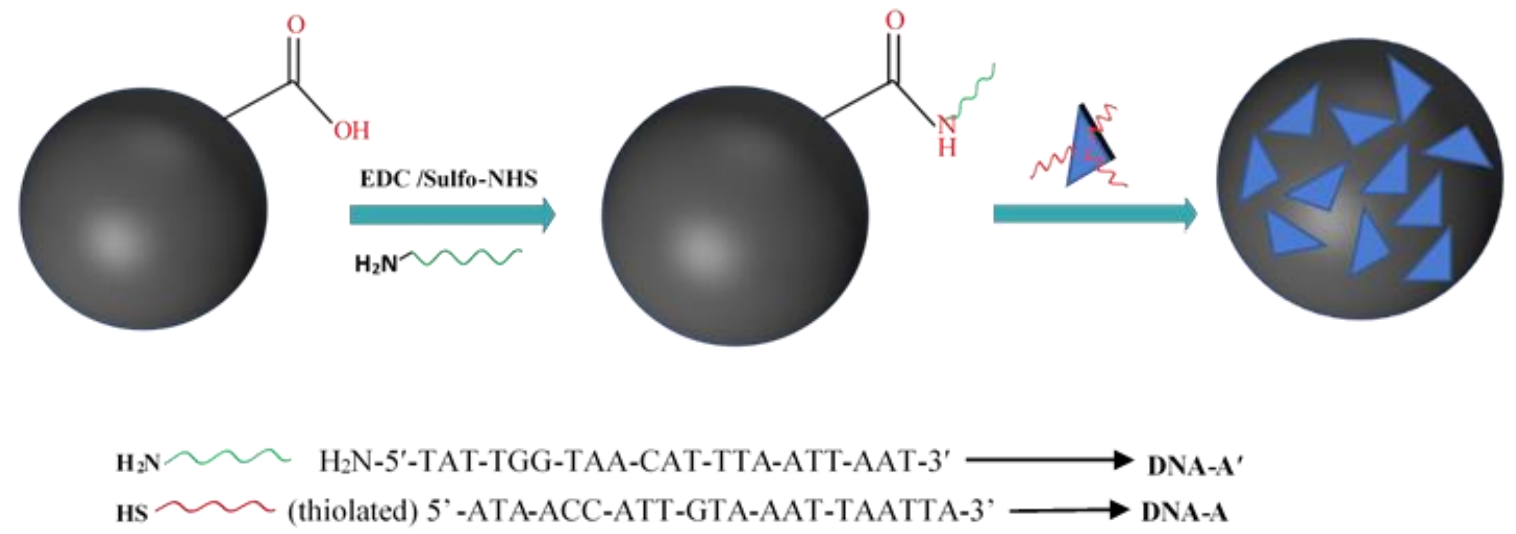

In the second step, nanoprisms that are densely functionalized with adenine rich ss-DNA, DNA-A were coated on thymine rich DNA-A' functionalized PS beads using complementary DNA-DNA interaction between DNA-A and DNA-A' ( see section 2.3.15 for details).

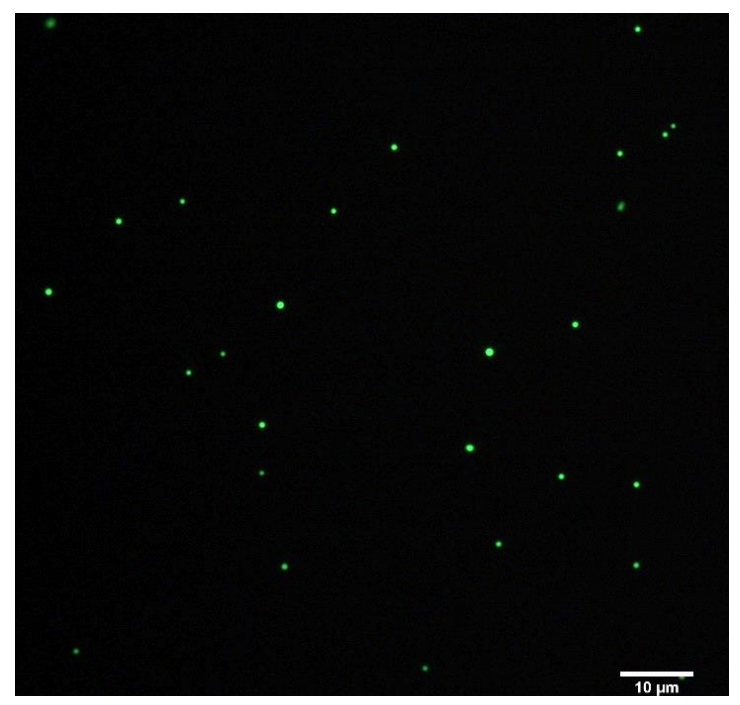

Figure 5.1: Fluorescent microscopy image of dispersed PS beads after DNA functionalization. 


\subsubsection{Characterization of PS beads/nanoprism conjugates}

The DNA-induced interaction between nanoprisms and PS beads resulted in the dense immobilization of nanoprisms on the PS bead surface, leading to the formation of PS beads/nanoprism conjugates, which were fully characterized by UV-Visible spectroscopy, dynamic light scattering (DLS) and SEM imaging. No PS beads/nanoprism conjugation was observed when uncoated PS beads were mixed with uncoated nanoprisms under the same conditions, as shown by SEM imaging (Figure 5.2), indicating specific complementary DNA-DNA interaction between PS beads and nanoprisms in PS beads/nanoprism conjugates.

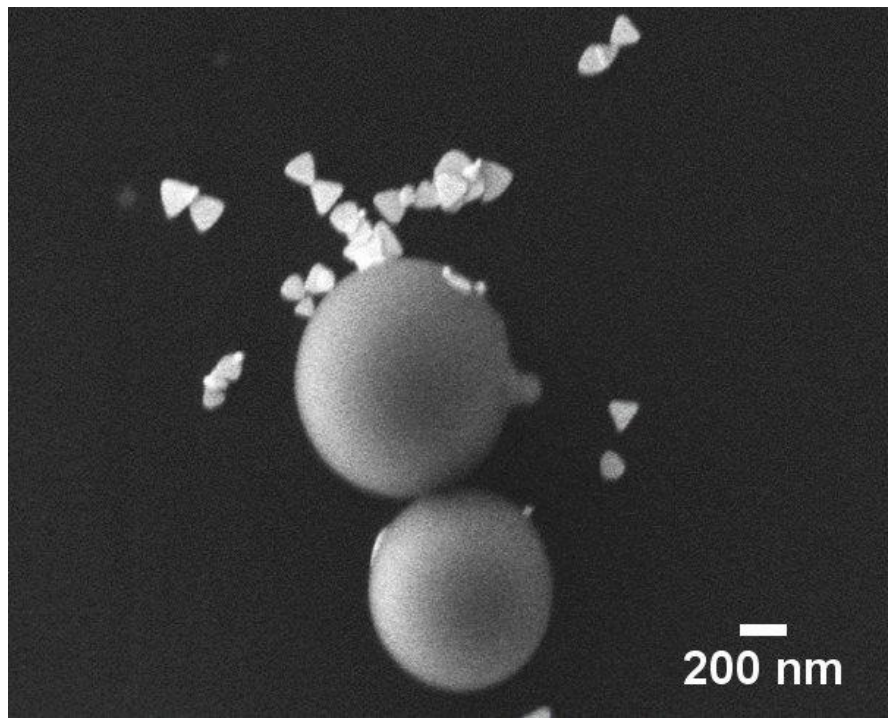

Figure 5.2: Representative SEM image showing very little binding between uncoated PS beads and uncoated gold nanoprisms.

To elucidate the effect of solution ionic strength on the DNA-mediated binding events between nanoprisms and PS beads, the conjugation of nanoprisms to PS 
beads was carried out at different salt concentrations, i.e. $0.1 \mathrm{M}, 0.2 \mathrm{M}$ and $0.3 \mathrm{M}$

$\mathrm{NaCl}$. Figure 5.3A shows the size distribution of PS beads/nanoprism conjugates at different salt concentrations and representative SEM images showing the successful immobilization of nanoprisms onto PS beads. The first peak at around $100 \mathrm{~nm}$ in the DLS graph denotes the presence of unbound nanoprisms while the peak at around $1 \mu \mathrm{m}$ hydrodynamic diameter represents PS beads/nanoprism conjugates that consists of nanoprism-coated single bead or dimers of beads as shown in SEM
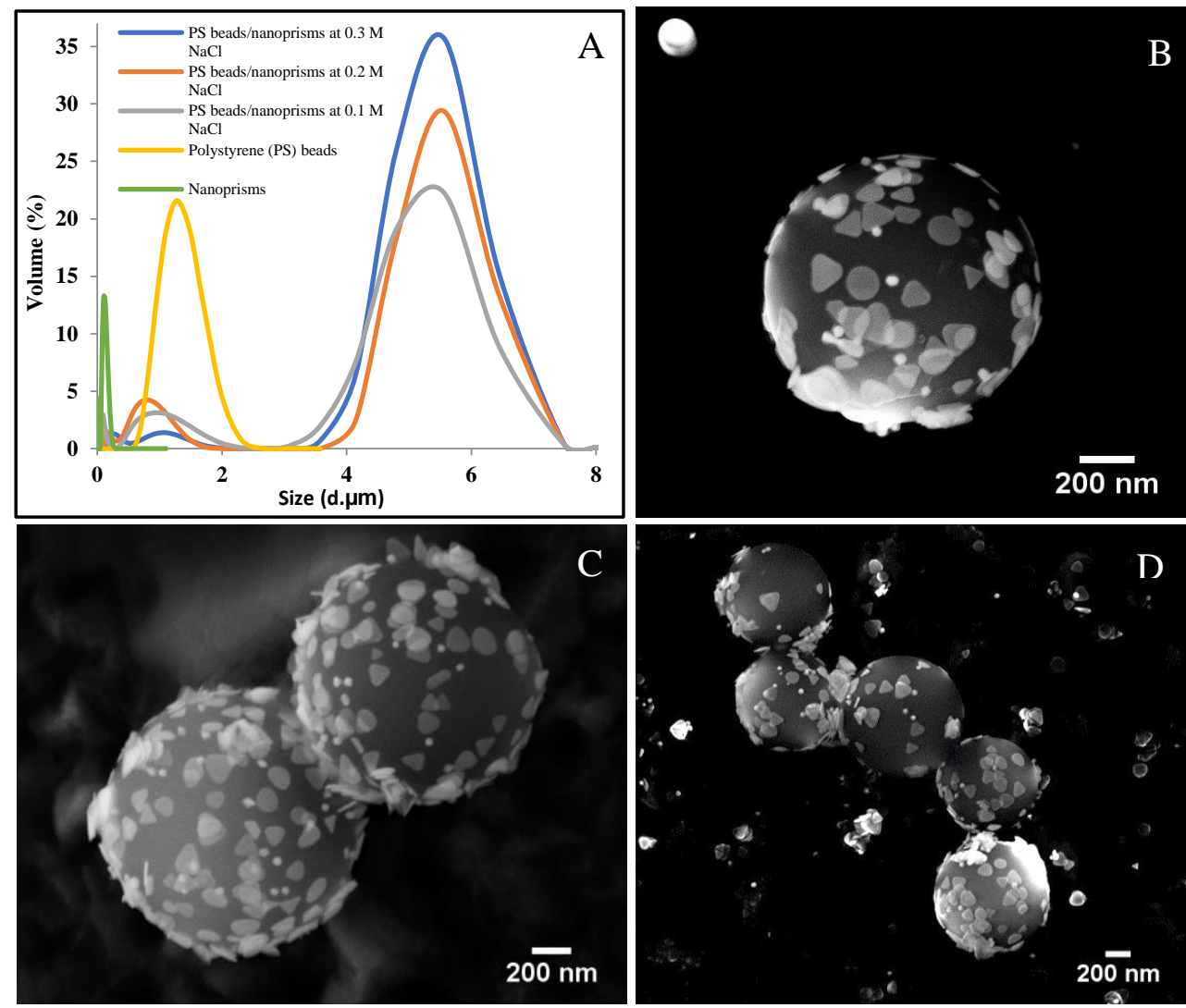

Figure 5.3: A) DLS size characterization of salt effect in DNA induced binding between nanoprisms and PS microbeads, B) \& C) Representative SEM image of PS beads/ nanoprisms conjugates, i.e. nanoprisms coated single or dimers of beads, and D) Representative SEM image of PS beads/nanoprisms conjugates, i.e. bead clusters. 
images, Figure 5.3B \&C , and the peak at around $5 \mu \mathrm{m}$ hydrodynamic diameter denotes PS beads/nanoprism conjugates, i.e. bead clusters, where 3-7 PS beads are bound together via the two major facets of nanoprisms, Figure 5.3D.

The relatively larger DLS peak at around $100 \mathrm{~nm}$ hydrodynamic diameter for the $0.1 \mathrm{M}$ ionic strength sample compared to samples at $0.2 \mathrm{M}$ and $0.3 \mathrm{M}$ ionic strength indicates that there are more unbound nanoprisms for $0.1 \mathrm{M}$ ionic strength and that the loading efficiency was indeed higher for $0.2 \mathrm{M}$ and $0.3 \mathrm{M}$ salt concentrations than $0.1 \mathrm{M}$ salt concentration. However, the quantitative effect of solution ionic strength on the loading density of nanoprisms was apparently insignificant and there was no particular trend observed from SEM images. Previous studies on DNA-mediated loading of gold nanospheres on polymer beads demonstrated that the loading density of nanoparticles significantly increases with increasing salt concentration. ${ }^{141}$ This difference in salt concentration dependence between the loading of anisotropic nanoprisms and nanospheres onto polymer beads could be attributed to the enhanced binding strength of nanoprisms, which is several million times higher than their nanosphere counterparts, stemming from the two major flat surfaces of prisms that can accommodate significantly more local DNA linker coating density than nanospheres. ${ }^{24}$

However ionic strength did play a significant role in the growth of PS beads/nanoprism clusters. As the salt concentration increased, the DLS peaks at larger diameters gradually increased, indicating the formation of a larger number of bead clusters at higher salt concentration. This is because high solution ionic 
strength sufficiently screens the long-range electrostatic repulsion between beads and facilitates close contact between neighboring beads for short range hydrogen bonding between complementary DNA strands.

\subsubsection{Salt effects on the conjugation of nanoprisms onto PS beads}

Figure 5.4 shows the UV-Vis absorption spectra of PS beads/nanoprisms conjugates at different salt concentrations. The SPR absorbance of nanoprisms in the conjugates exhibits a significant red shift from the SPR absorbance of nanoprisms at $895 \mathrm{~nm}$, Figure 5.4A. The red shift in absorption band upon conjugation of nanoprisms to PS beads can be attributed to the interaction of nanoprisms with the PS beads as well as neighboring nanoprisms on the PS beads surface. Absorption spectra also reveal the effect of ionic strength on the formation of PS beads/nanoprisms conjugates. For instance, the SPR band of nanoprisms
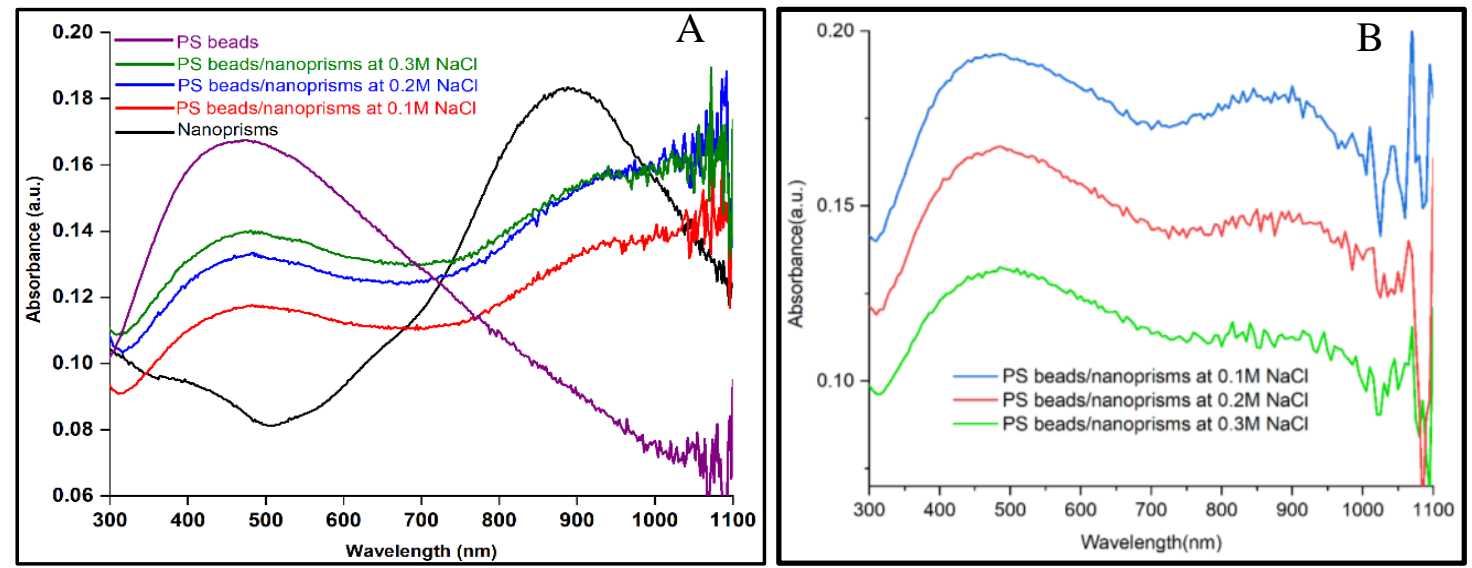

Figure 5.4: A) UV-Vis characterization of salt effect in DNA induced binding between larger nanoprisms and PS beads, B) UV-Vis characterization of salt effect in DNA induced binding between smaller nanoprisms and PS beads. 
were increasingly red shifted with increasing salt concentration from $0.1 \mathrm{M}$ to 0.3

M, Figure 5.4A \& B, indicating that higher ionic strength increases the formation of PS beads/nanoprisms cluster.

\subsubsection{Fluorescence quenching of PS beads at different salt concentration}

Gold nanoparticles can function as highly efficient fluorescence quenchers when the fluorophore is placed in proximity of nanoparticles. ${ }^{275-278}$ This phenomenon of fluorescence quenching has been utilized for molecular sensing applications $^{279}$ and energy transfer assays for the detection of biomolecules. ${ }^{280}$ Moreover, conditional quenching or selective quenching of fluorescence can find applications in negative sensing. ${ }^{281}$ The efficiency of fluorescence quenching by the nanoparticle is dependent on the distance between nanoparticle and fluorescent object, i.e. fluorophore and chromophore. Dulkeith, et al. investigated the mechanism of fluorescence quenching of cy5 for distances ranging from 2-16 nm between the nanoparticle and cy5. ${ }^{282}$ They showed that at all examined distances, the fluorescence intensity or efficiency of cy5 was reduced due to a reduced radiative decay rate of cy5. In this study, we used two 21-mer complementary ssDNA strands, which are intertwined to form double helices in PS beads/nanoprism conjugates. Therefore, the approximate distance between the nanoprisms and PS beads would be around $7 \mathrm{~nm}$, excluding salt-induced contraction of DNA. At all salt concentrations, the fluorescence intensity of the PS beads in PS beads/nanoprism conjugates was significantly reduced compared to the intensity of uncoated PS beads of the same concentrations. 
The emission spectra of fluorescent PS beads in PS beads/nanoprism conjugates at various salt concentrations is shown in Figure 5.5. For all salt concentrations, the fluorescence of PS beads was almost quenched, and the intensity was reduced by almost $78 \%$ due to damping of PS beads' molecular dipole by the attached nanoprisms. ${ }^{283}$ This could be due to phase induced suppression of the radiative decay rate of the fluorescent PS beads, which are tangentially oriented to the nanoprisms surfaces. ${ }^{282,284}$ The effect of salt

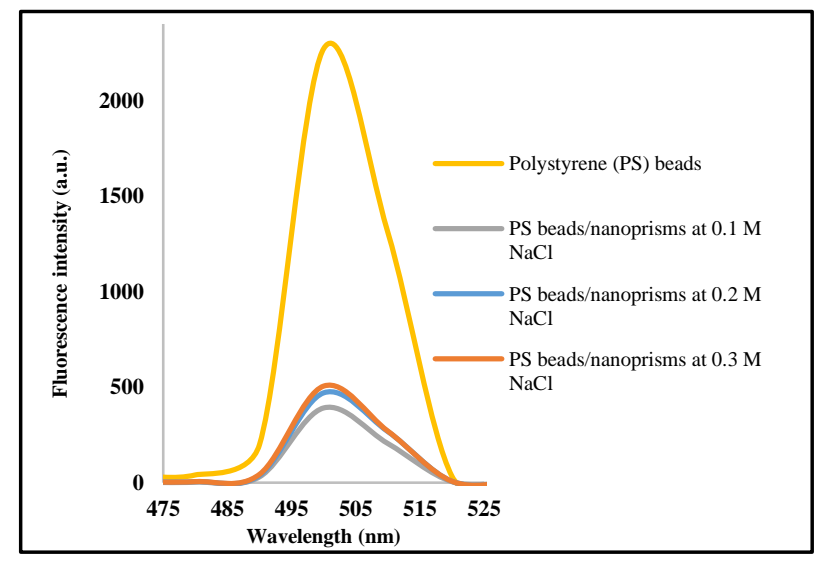

Figure 5.5: Fluorescence quenching of PS beads at different salt concentration.

concentration on the fluorescence quenching was insignificant, which could be attributed to the lack of salt effect on the loading density of nanoprisms onto PS beads.

\subsubsection{Formation of 3D PS beads/Nanoprism aggregates}

DNA-linked nanoparticle and microparticle aggregates exhibit cooperative melting behavior stemming from short range duplex to duplex interactions. ${ }^{285}$ Upon 
heating above a characteristic temperature, which dissociates duplex DNA structures linking the particles into two complementary DNA strands, DNAinduced particle aggregates show a sharp melting transition, which is an indicative of dense functionalization of particles in DNA mediated particle assembly systems. In order to determine the characteristic melting temperature, PS beads/nanoprisms conjugates were heated from $45-90^{\circ} \mathrm{C}$ at a ramp rate of $0.25^{\circ} \mathrm{C} / \mathrm{min}$. During the heating process, the change in absorbance at surface plasmon resonance band (895 $\mathrm{nm}$ ) of nanoprisms was monitored as a function of temperature. A control experiment was also carried out by similarly annealing a solution containing uncoated nanoprisms and uncoated PS beads. The sharp melting profile for the PS beads/nanoprism conjugates (Figure 5.6A), as monitored via UV-vis spectroscopy, and the lack of such profile for the control experiment as shown in Figure 5.6B
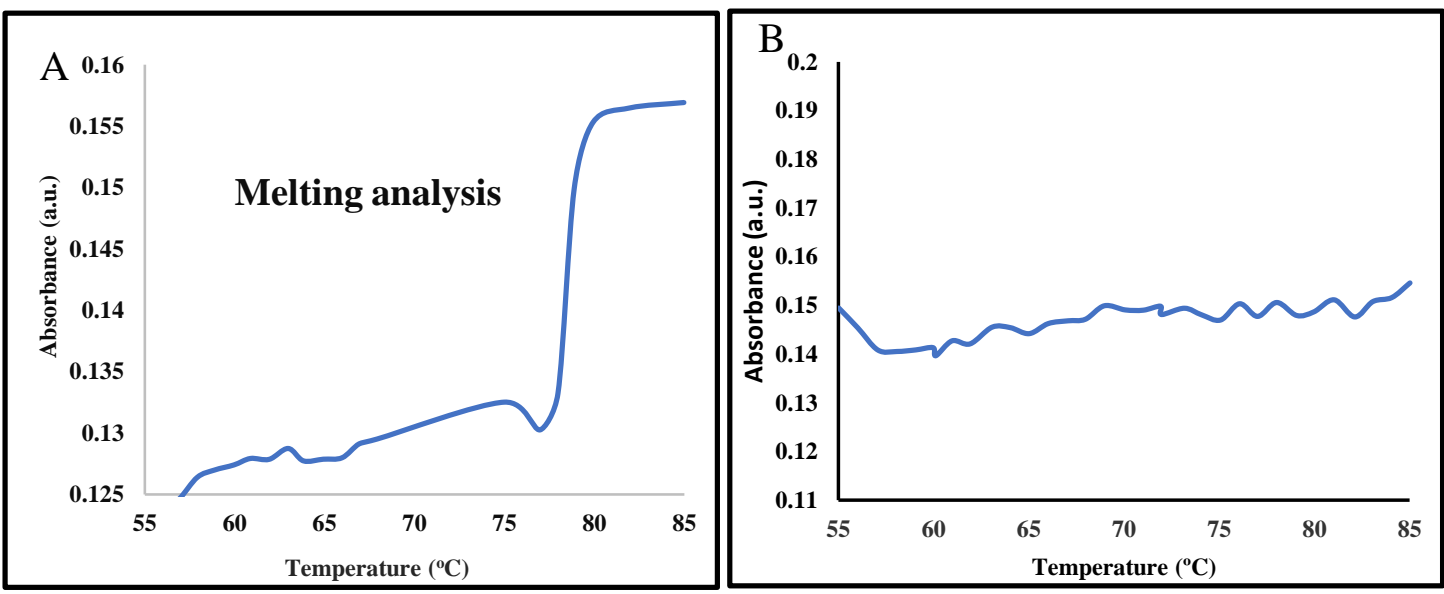

Figure 5.6: A) Melting profile of PS beads/nanoprism conjugates, monitored at $895 \mathrm{~nm}$ (the SPR of nanoprisms), B) Melting profile of control experiment, i.e. mixture of uncoated nanoprisms and uncoated PS beads in a hybridization buffer, monitored at $895 \mathrm{~nm}$. 
confirmed the dense functionalization of DNA on nanoprisms and PS beads and DNA-mediated interaction between PS beads and nanoprisms in PS beads/nanoprism conjugates. Dehybridization of DNA linked PS beads/nanoprism conjugates occurred over a narrow temperature range and the melting point was determined to be the inflection point at $78^{\circ} \mathrm{C}$ of the melting curve, approximately $37^{\circ} \mathrm{C}$ higher than $\mathrm{T}_{\mathrm{m}}$ of the DNA linkers. This significant increase in $\mathrm{T}_{\mathrm{m}}$ of PS beads/nanoprisms aggregates could be attributed to enhanced binding between nanoprisms and PS beads, and the formation of beads' cluster.

In order to create a large area of 3D homogenous SERS substrates, we adopted a DNA-induced nanoparticle/polymer bead crystallization approach, i.e. thermal annealing followed by slow cooling. Initially formed PS beads/nanoprism conjugates were heated to $65-70^{\circ} \mathrm{C}$, which is below the melting point of the PS beads/nanoprism conjugates, yet higher than the melting point of the DNA linkers. Thermal annealing below the melting point of the conjugates ensures the intactness of PS beads/nanoprism conjugates, while slow cooling and heating above the $\mathrm{T}_{\mathrm{m}}$ of the DNA strands allows the long range ordering of PS beads via DNA induced interaction between DNA-A coated nanoprisms and DNA-A' coated PS beads, yielding a large ensemble/stack of PS beads (PS beads/nanoprism aggregates). The resulting ensemble has a diameter ranging from 10-15 $\mu \mathrm{m}$ and consists of 25-50 PS beads that are bound through nanoprisms and DNA, Figure 5.7A\&B.

Each bead in the aggregates contains a number of closely spaced nanoprisms with various orientations such as tip-to-tip bowtie structures, tip-to-edge, edge-to- 
edge as well as interstitial orientations, with gap distances between prisms ranging from 1-20 nm.
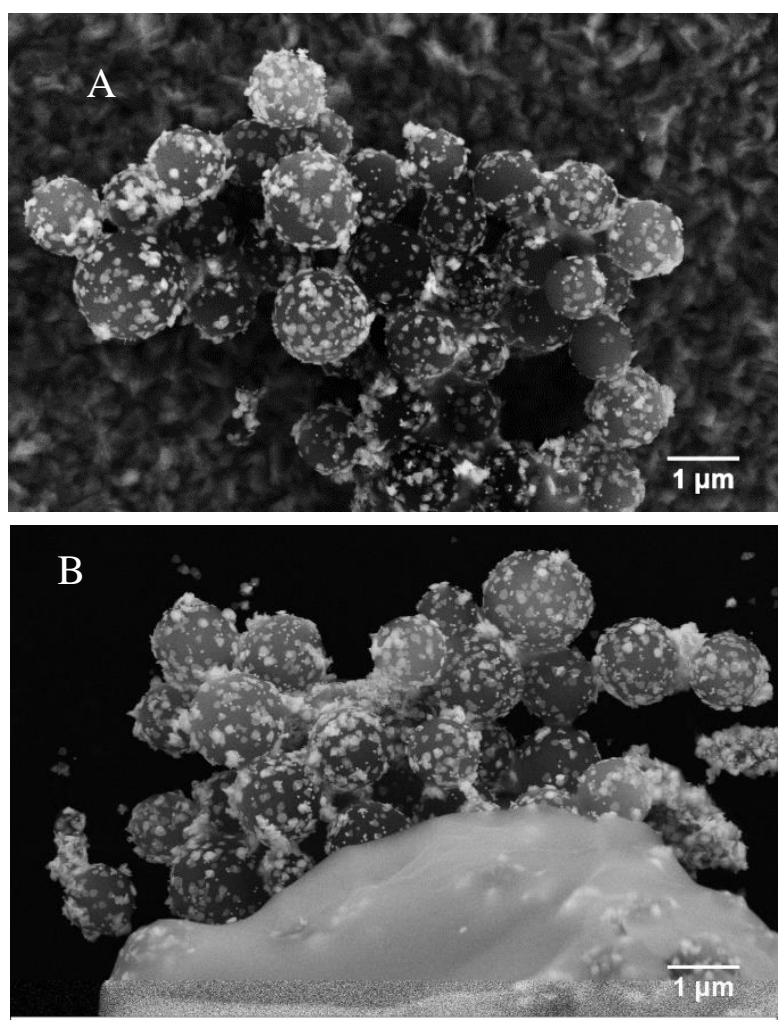

Figure 5.7: A) \&B) Representative SEM images of 3D PS beads /nanoprisms aggregates.

Depending on the gap and orientations, various arrangements of nanoprisms on the PS bead surfaces lead to varying degrees of plasmonic coupling and electromagnetic field enhancement in the vicinity of the nanoprisms' sharp edges and tips. Thus, the junctions of a large number of closely positioned nanoprisms in the 3D stacks of PS beads promotes the formation of high densities of plasmonic hot spots that could lead to strong SERS enhancement. 


\subsubsection{SERS activity of 3D PS beads/Nanoprism aggregates}

The performance of 3D PS beads/nanoprism aggregates as a SERS substrate was evaluated using the common Raman reporter molecule methylene blue (MB). MB is chosen as the model compound for SERS analysis because of its well-known characteristic Raman bands. Figure 5.8A shows the Raman spectra of 3D PS beads/nanoprism aggregate substrates without $\mathrm{MB}$ and with a $10^{-4} \mathrm{M}$ aqueous solution of MB on three substrates: PS beads aggregates, glass substrate and 3D PS beads/nanoprism aggregates. All the spectra were collected under the same conditions, using an excitation wavelength of $633 \mathrm{~nm}$.

A

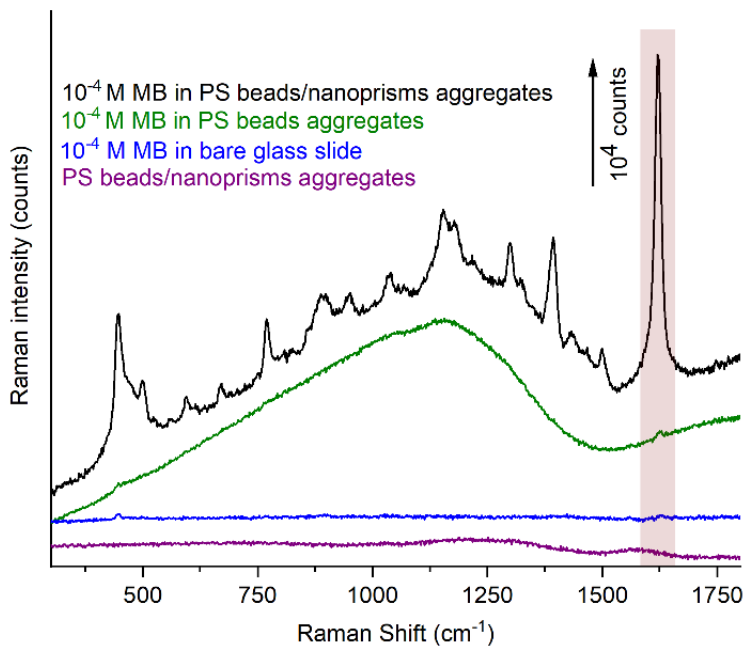

B

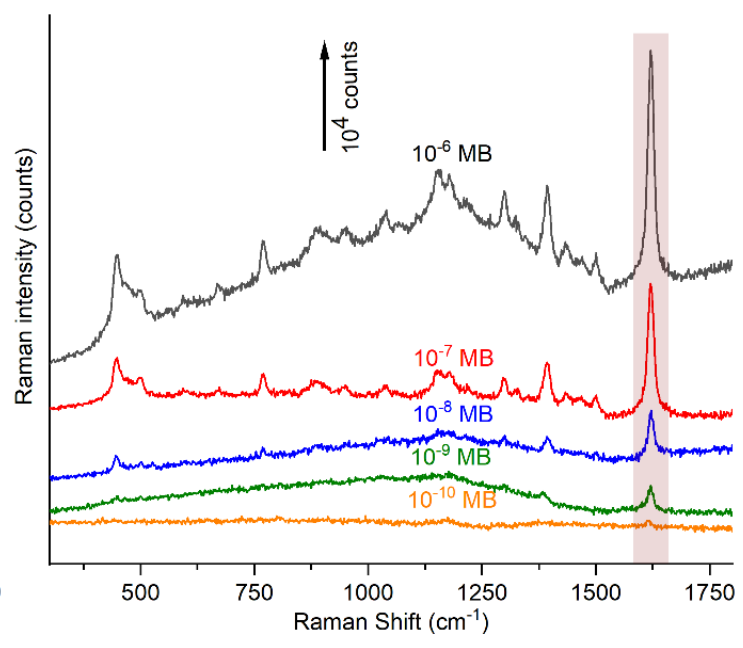

Figure 5.8: A) Raman spectrum of PS beads/nanoprism substrate (green) and SERS spectra of MB adsorbed on glass substrate (blue), PS bead aggregates (Red), \& PS beads/nanoprism aggregates (black), B) SERS spectra of MB on 3D PS beads/nanoprism aggregates at different concentrations. 
Due to smaller Raman cross section, only two characteristic peaks with weak intensities were observed in the Raman spectrum for the $10^{-4} \mathrm{M} \mathrm{MB}$ on both glass substrate and PS beads aggregates. On the other hand, all the characteristic peaks of MB were observed with good signal to noise ratio in the SERS spectrum recorded for the same concentration of MB on the 3D PS beads/nanoprism aggregate substrates ( see section for MB peak assignments). These peaks were not observed for the Raman analysis of the SERS substrate and glass substrate without MB, indicating that they originate from the MB. The strong signal enhancement for the band at $1621 \mathrm{~cm}^{-1}$ indicates a favorable orientation of probe molecules on the nanoprism surface, presumably through the fused phenyl and thiazine rings of the probe molecule. ${ }^{253}$

While Raman analysis of $10^{-4} \mathrm{M}$ MB on PS beads/nanoprism aggregates substrate reveals a highly structured spectrum with well resolved characteristic peaks of MB, only two peaks with weak intensities were recorded for the same concentration of MB on PS bead aggregates and glass substrates, indicating that the 3D PS beads/nanoprism aggregates are highly SERS active. The strong SERS enhancement for the 3D aggregates is attributed to the presence of plasmonic hot spots due to enhanced coupling and field enhancement from a close packed arrangement of nanoprisms. Notably, the Raman spectrum of MB on PS bead aggregates is masked by the autofluorescence of the PS beads, resulting in a larger fluorescence background and weaker Raman signal in the spectra. This is due to the fact that the laser excitation energy is close to the electronic transition energies of 
PS beads. ${ }^{286}$ However, due to quenching of PS beads' fluorescence by the nanoprisms, the Raman spectra of MB on 3D PS beads/nanoprism aggregate and 3D PS beads/nanoprism aggregates themselves didn't show fluorescence background. The molecular detection limit of 3D PS beads/nanoprism aggregates was determined by recording SERS spectra of MB at different concentration varying from $10^{-6}$ to $10^{-10} \mathrm{M}$, Figure $\mathbf{5 . 8 B}$. The intensity of SERS peaks for MB expectedly decreased as the concentration of MB decreased. An obvious SERS peaks at $1621 \mathrm{~cm}^{-1}$ were still clearly visible at a very low MB concentration of $10^{-10}$ M, indicating a high detection sensitivity of 3D PS beads/nanoprism aggregates.

As compared to SERS enhancement of PS beads/nanoprism aggregates, the SERS intensity of $10^{-4} \mathrm{M} \mathrm{MB}$, as shown in Figure 5.9, obtained using randomly

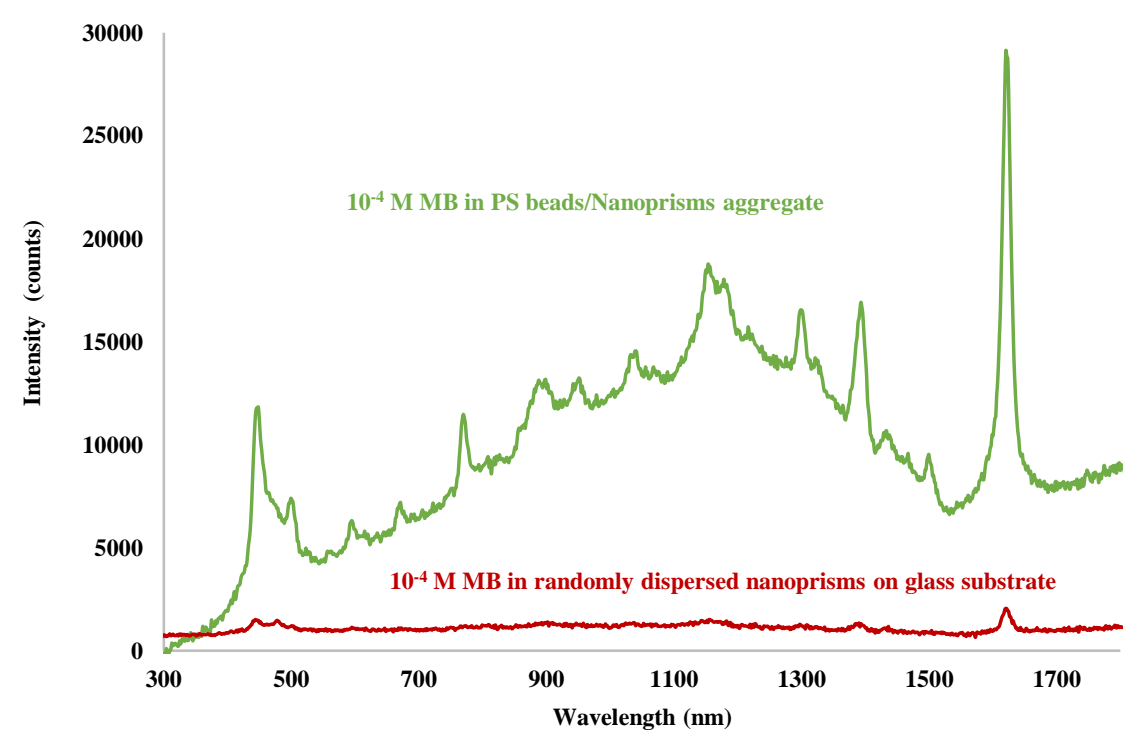

Figure 5.9: SERS intensity of $10^{-4} \mathrm{M} M B$ using 3D PS beads/nanoprism aggregates (green) and randomly dispersed nanoprisms (dark red). 
dispersed nanoprisms on glass substrate was substantially lower, which highlights the importance of densely packed arrangement of nanoprisms for high SERS efficiency.

To assess the reproducibility in SERS response of PS beads/nanoprism aggregates, SERS intensity at $1621 \mathrm{~cm}^{-1}$ was measured for $10^{-7} \mathrm{M}$ MB from 15 different PS beads/nanoprisms aggregates, Figure 5.10. The average signal intensity at $1621 \mathrm{~cm}^{-1}$ for the PS beads/nanoprism aggregates was 3580.4 counts with a coefficient of variation $(\mathrm{CV})$ of only $18 \%$, indicating that good reproducibility of the PS beads/nanoprism aggregates as a SERS substrate.

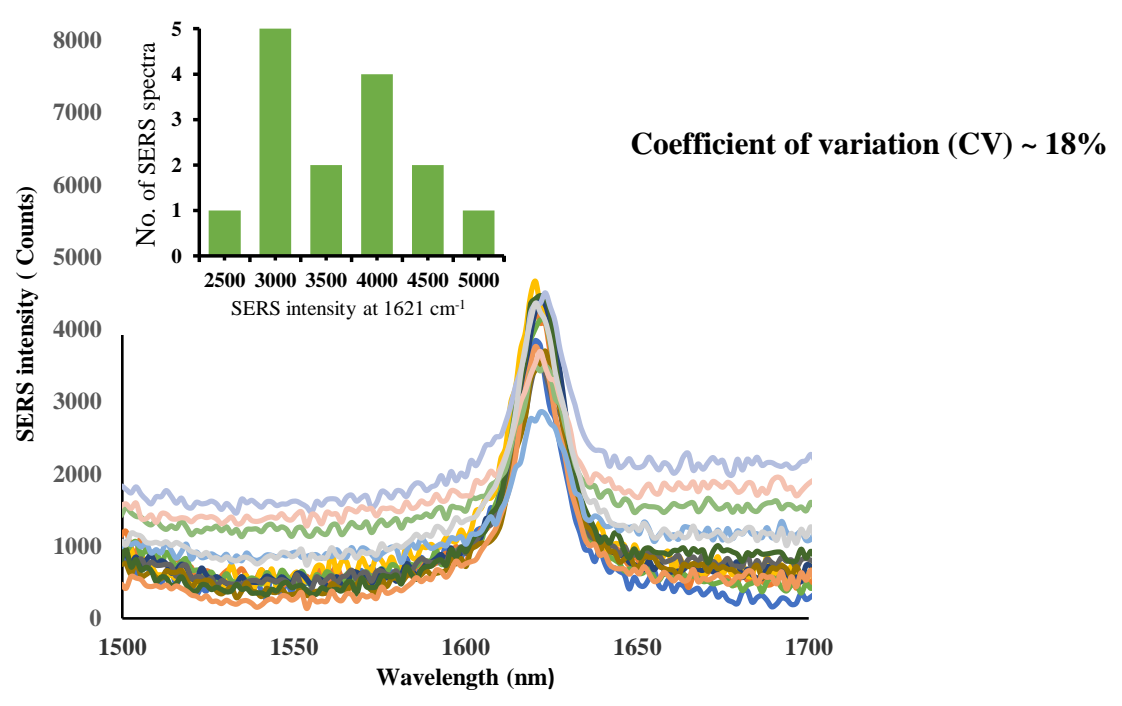

Figure 5.10: Spot-to-spot variation in SERS intensity of MB $\left(10^{-7} \mathrm{M}\right)$ at $1621 \mathrm{~cm}^{-1}$ for the PS beads/nanoprism aggregates (SERS measurements were collected from 15 different spots). 
The SERS enhancement factor (EF) of 3D PS beads/nanoprism aggregates was calculated from the SERS intensity of the prominent band at $1621 \mathrm{~cm}-1\left(10^{-7} \mathrm{M}\right.$ $\mathrm{MB})$ and Raman intensity of the corresponding band $\left(10^{-4} \mathrm{M} \mathrm{MB}\right)$, considering bare glass substrates as the reference (see Experimental details for details). The SERS enhancement of MB on the 3D PS beads/nanoprism substrate was assessed by calculating the enhancement factor $(\mathrm{EF})$ using the following equation.

$$
E F=\frac{I_{S E R S} / N_{S E R S}}{I_{\text {Bulk }} / N_{\text {Bulk }}}
$$

where $\mathrm{I}_{\mathrm{SERS}}$ and $\mathrm{I}_{\mathrm{BULK}}$ refer to the peak intensity at $1621 \mathrm{~cm}^{-1}$ in the SERS spectrum of $10^{-7} \mathrm{M} \mathrm{MB}$ on the PS beads/nanoprism substrate and bulk $10^{-4} \mathrm{M} \mathrm{MB}$ on the reference glass substrate, respectively. $\mathrm{N}_{\text {SERS }}$ and $\mathrm{N}_{\text {BULK }}$ refers to the number of adsorbed MB molecules within the laser spot on the 3D substrate and the number of adsorbed MB molecules in a bulk sample (glass substrate), respectively.

\section{Number of MB molecules excited for Raman ( $\left.\mathrm{N}_{\text {Bulk }}\right)$ :}

Deposition $10 \mathrm{uL}$ of $10^{-4} \mathrm{M}$ of $\mathrm{MB}$ produced a spot of approximately $5 \mathrm{~mm}$ diameter on the glass slide. 
Table 5.1: Calculation of the number of MB molecules excited for Raman

Area of MB spot size $=0.13 \mathrm{~cm}^{2}$

Area of laser spot size $=0.83 \mu m^{2}$

Initial bulk $\mathrm{MB}$ moles $=1 \times 10^{-9}$

Number of bulk MB molecules irradiated, $\mathrm{N}_{\text {Bulk }} \mathrm{MB}$ molecules $=2.6 \times 10^{7} \mathrm{MB}$ molecules

\section{Number of MB molecules excited for SERS (NsERS):}

Deposition $10 \mathrm{uL}$ of $10^{-7} \mathrm{M}$ of MB produced a spot of approximately $5 \mathrm{~mm}$ diameter on the 3D SERS substrate

Table 5.2: Calculation of the number of MB molecules excited for SERS

Initial bulk MB within 3D substrate moles $=1 \times 10^{-12}$ moles

Number of estimated nanoprisms within laser spot $\approx 20$ (Estimated from SEM image)

Surface area of a single nanoprism $=\frac{\sqrt{3 a^{2}}}{2}+3 a h=0.01 \mu m^{2} \quad$ (edge length, $a \approx$ $91.25 \mathrm{~nm}$, thickness, $h \approx 10 \mathrm{~nm}$ )

Number of MB molecules adsorbed per nanoprism MB molecules $=306$ molecules

Number of MB molecules excited for SERS, $\mathrm{N}_{\mathrm{SERS}} \mathrm{MB}$ molecules $=6.1 \times 10^{3} \mathrm{MB}$ molecules 
The SERS signal intensity at $1621 \mathrm{~cm}^{-1}$ is $481.4 \mathrm{cps}$ and normal Raman signal intensity at $1621 \mathrm{~cm}^{-1}$ is $18.5 \mathrm{cps}$.

Therefore, $E F=\frac{481.4 / 6.1 \times 10^{3}}{18.5 / 2.6 \times 10^{7}}=1.09 \times 10^{5}$

The EF value for the 3D SERS substrate is estimated to be $1.09 \times 10^{5}$. The SERS performance of the 3D SERS substrate is comparable to or better than that of other nanoparticle-based plasmonic SERS substrates for the SERS analysis of MB. ${ }^{251-252 \text {, }}$ $254-256$

\subsection{Conclusion}

In conclusion, we have demonstrated a DNA mediated self-assembly strategy for the fabrication of large area homogenous SERS substrates, i.e. PS beads/nanoprism aggregates, from PS beads and gold nanoprisms. The conjugation of nanoprisms to PS beads was carried out using DNA-DNA hybridizations and the effect of solution ionic strength on the conjugation was investigated. No clear trend was observed for the effect of salt concentration on the loading density of nanoprisms on PS beads, but the size of nanoprisms loaded PS beads clusters increased with the increasing salt concentration as evidenced by the increasing red shift of SPR of both nanoprisms and DLS size distribution. The fluorescence intensity of fluorescent PS beads was reduced by $78 \%$ due to quenching effects of the nanoprisms conjugated to PS beads.

A SERS substrate was fabricated by assembling the initially formed PS bead/nanoprism conjugates into a large 3D PS beads/nanoprism aggregates via annealing the conjugates below the $\mathrm{T}_{\mathrm{m}}\left(78^{\circ} \mathrm{C}\right)$, followed by slow cooling that 
facilitated the long-range interaction between PS beads and nanoprisms. The average size of PS beads/nanoprism aggregates was found to be $10-15 \mu \mathrm{M}$ in diameter and each aggregate consisted of 20-50 beads, where a large density of nanoprisms are arranged on the PS bead surfaces in various directions with varying interparticle distances, leading to the formation 3D plasmonic fields with a high density of plasmonic hot spots. The presence of such a high-density of hot spots led to homogeneous and high SERS enhancement. SERS measurements of methylene blue probe molecules on the substrates demonstrated excellent SERS enhancement with a limit of detection as low as $10^{-10} \mathrm{M}$ concentration of MB. We believe that the conjugation and self-assembly approach we demonstrated here could be utilized for a number of applications including negative fluorescence-based detection and SERS based molecular sensing. 


\section{CHAPTER 6}

\section{SYNTHESIS AND OPTICAL PROPERTIES OF GOLD NANOPRISM DIMERS AND TRIMERS}




\subsection{Introduction}

In the quest for various novel plasmonic application, directed self-assembly emerges as a promising route to organize few nanoscale objects into small clusters known as 'artificial molecule'. ${ }^{287}$ The surface plasmons of nanoparticle in these assembled molecule exhibits plasmonic hybridization similar to atomic orbitals. ${ }^{288}$ This property prompts the design and construction of various artificial plasmonic nano-systems, giving rise to many intriguing phenomenon and emergent applications in plasmonic ruler, ${ }^{289}$ non-linear optics, ${ }^{290}$ and plasmonic lasers ${ }^{291}$. In particular, plasmonic Fano resonance has drawn significant attention due to its characteristic narrow and deep line shape and asymmetric spectral profile, which is inherently sensitive to the change in local dielectric environment. ${ }^{292-293}$ Moreover, Fano resonance shows a dark sub-radiant mode and bright-super radiant mode. ${ }^{294}$ These properties endow plasmonic metamaterials with Fano resonance many promising applications including high figure of merit (FoM) refractive index sensing, ${ }^{295}$ electromagnetically induced transparency, ${ }^{296}$ light trapping, ${ }^{297}$ and energy storage ${ }^{298}$. Various plasmonic nanostructures such as non-concentric ring-disk cavities, ${ }^{299}$ metal nanoparticle oligomers, ${ }^{300}$ nanofilm, ${ }^{301}$ and nano-shells ${ }^{302}$ have been designed and fabricated to explore Fano resonance. In general, anisotropic nanomaterials with reduced symmetry are better suited to investigate Fano resonance. ${ }^{294,303}$

Various top-down lithography and bottom-up self-assembly approaches have been introduced for arranging nanoscale objects into plasmonic clusters known with high precision and tunable interparticle distance. High precision lithography in combination 
with layer by layer stacking techniques have been used to place a single gold nanorod in between two pairs of nanorods for a 3D plasmonic ruler application. ${ }^{304}$ The combination of top down lithography and bottom up DNA-based assembly has also been reported to construct superlattices of nanoparticle heterotrimers, which exhibited solvent responsive broadband absorption. ${ }^{305}$ Additionally, the side-to-side and end-toend assembly of gold nanorods has been accomplished using several different selfassembly approaches, including electrostatic ${ }^{306-307}$ and covalent interactions, ${ }^{125}$ and use of aromatic dithiol linkers. ${ }^{308}$ Despite the significant advances in the organization of nanoparticles into, the precise arrangement of anisotropic building blocks into composite plasmonic nanostructures on the nanoscale such as dimer and trimer remains a key challenge.

The use of DNA has been proven to be a powerful tool for directed assembly of nanoparticles to construct highly functional and ordered nanostructures. ${ }^{169,216}$ As discussed in Chapter 4 and Chapter 5, the simple design rules and sequence specific interactions of DNA render unprecedented tunability that has been utilized for assembling nanoparticle building blocks into various 1D, 2D and 3D nanoparticle superstructures with different lattice symmetry and well-defined crystallization habits. ${ }^{29,108,170,309-310}$ Moreover, the programmability of DNA bonds also allows tunability in the interparticle distance from $3 \mathrm{~nm}$ to above $130 \mathrm{~nm}$ by simply just changing the number of base pairs. In contrast to a diverse set of DNA based nanostructures that involve symmetric and dense DNA functionalization of nanoparticles, the study on asymmetric functionalization of nanoparticles that 
facilitates the formation of plasmonic dimers and trimers of nanoparticles, in particular those of anisotropic nanoparticles has been very limited. The ability to introduce valency into anisotropic nanoparticles and construct designer novel complex plasmonic nanostructures would present a significant step forward in expanding the range of potential nanomaterials.

Here we describe a DNA-directed self-assembly of asymmetrically functionalized Janus gold nanoprisms to construct plasmonic dimers and trimers. We first functionalized the major facets of nanoprisms with DNA and PEG to create Janus nanoprisms. This asymmetric functionalization allowed nanoprisms to interact through only DNA-containing facets via Watson crick base pairing of complementary DNA sequences, as PEG containing facets don't exhibit any interaction. We then characterized the assembly of nanoprisms into dimers and trimer with SEM and UVVis spectroscopy.

Additionally, we also studied the plasmonic response of individual dimers and trimers by a combination of SEM, darkfield microscopy imaging and single particle hyperspectral spectroscopy. Single particle scattering spectra of dimers and trimers exhibit significant red shifts and reproducible spectral patterns, which possess a narrow and asymmetric dip at around $817 \mathrm{~nm}$, commonly known as "Fano resonance". 311 


\subsection{Results and discussion}

\subsubsection{Design and synthesis of gold nanoprism dimers and trimers}

The directed self-assembly approach for the synthesis of gold nanoprisms dimers and trimers was based on the DNA-mediated interaction of asymmetrically functionalized Janus gold nanoprisms ( see section 2.3.18 for details) .This asymmetric functionalization strategy allows facet selective DNA induced interactions leading to asymmetric assembly of nanoprisms, and resulted in the formation of gold nanoprism dimers and trimers in solutions. To accomplish facet selective interaction, we first synthesized Janus gold nanoprisms by controllably placing thiolated PEG on one facet and thiolated complementary DNA strands (adenine rich DNA-A and thymine rich DNA- $\mathbf{A}^{\prime \prime}$ ) on the other facet of the nanoprisms. The non-reactivity of PEG surfaces and complementary DNA interactions between DNA-A in PEG $\mid$ DNA-A and DNA-A" in PEG $\mid$ DNA- A" Janus nanoprisms enabled the formation of nanoprism dimers and the interaction between DNA- A" in DNA- $\mathbf{A}^{\prime \prime} \mid$ DNA- $\mathbf{A}^{\prime \prime}$ nanoprisms and DNA-A in PEG | DNA-A nanoprisms led to assembly of nanoprism trimers. 


\subsubsection{Characterization of Janus nanoprisms}

We synthesized two sets of Janus nanoprisms following literature precedent, ${ }^{165}$ where one set consist of PEG and DNA-A, while the other set contains PEG and DNA-A".

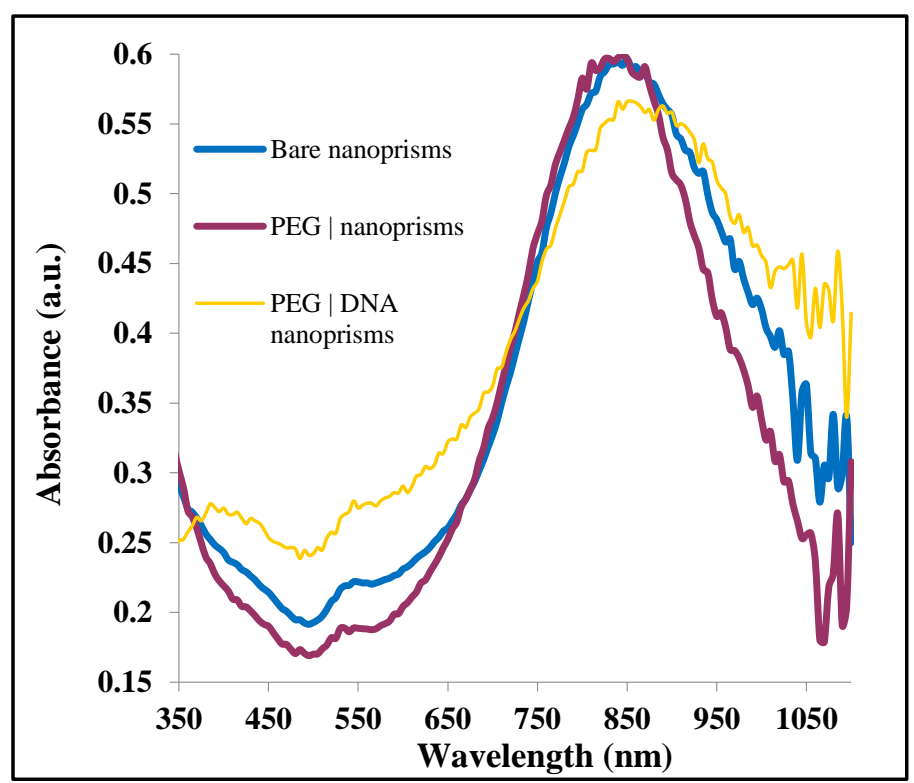

Figure 6.1: UV-vis spectra of nanoprisms before (blue) and after surface modification with PEG (dark red) and DNA (yellow).

Figure 6.1 shows UV-Vis spectra for the synthesis of Janus nanoprisms. The surface plasmon resonance maxima at $840 \mathrm{~nm}$ is redshifted by $14 \mathrm{~nm}$ after PEG functionalization on one facet of the nanoprisms and then another $11 \mathrm{~nm}$ after the attachment of DNA on the other facet. This is due to the change in the local refractive index resulting from surface modification with PEG and DNA. 


\subsubsection{Characterization of nanoprisms dimers and trimers}

The formation of dimers and trimers was confirmed and characterized by SEM microscopy and UV-Vis absorption spectra. In order to accomplish nanoprism dimer synthesis PEG | DNA-A and PEG | DNA- A" Janus nanoprisms were mixed in a DNA hybridization buffer in 1: 1 molar ratio. As illustrated in the SEM microscopy images the primary product for the reaction between equimolar Janus PEG |DNA-A and Janus PEG | DNA-A" nanoprisms was dimers, Figure 6.2. However, there were also singlets, triplets and multiplets. From the SEM images, the yield of nanoprism dimers was estimated to be around $60 \%$, which is comparable to the dimer assembly of other nanoparticles reported in the literature. ${ }^{145,312-313}$ Control studies were carried out by allowing two sets of complementary nanoprisms coated with only DNA (DNA-A or DNA-A") to react under the same experimental condition, Figure 6.3A, which resulted in the formation of 1D nanoprism stacks and taking PEG coated particles in DNA hybridization buffer, which didn't exhibit any interaction, Figure 6.3B.

Furthermore, the presence of mixed dual coatings (both PEG and DNA) instead of mutually exclusive Janus surface coatings i.e. either DNA or PEG, generated some random stacks of nanoprisms and unreacted nanoprisms, as shown in Figure 6.3C. 


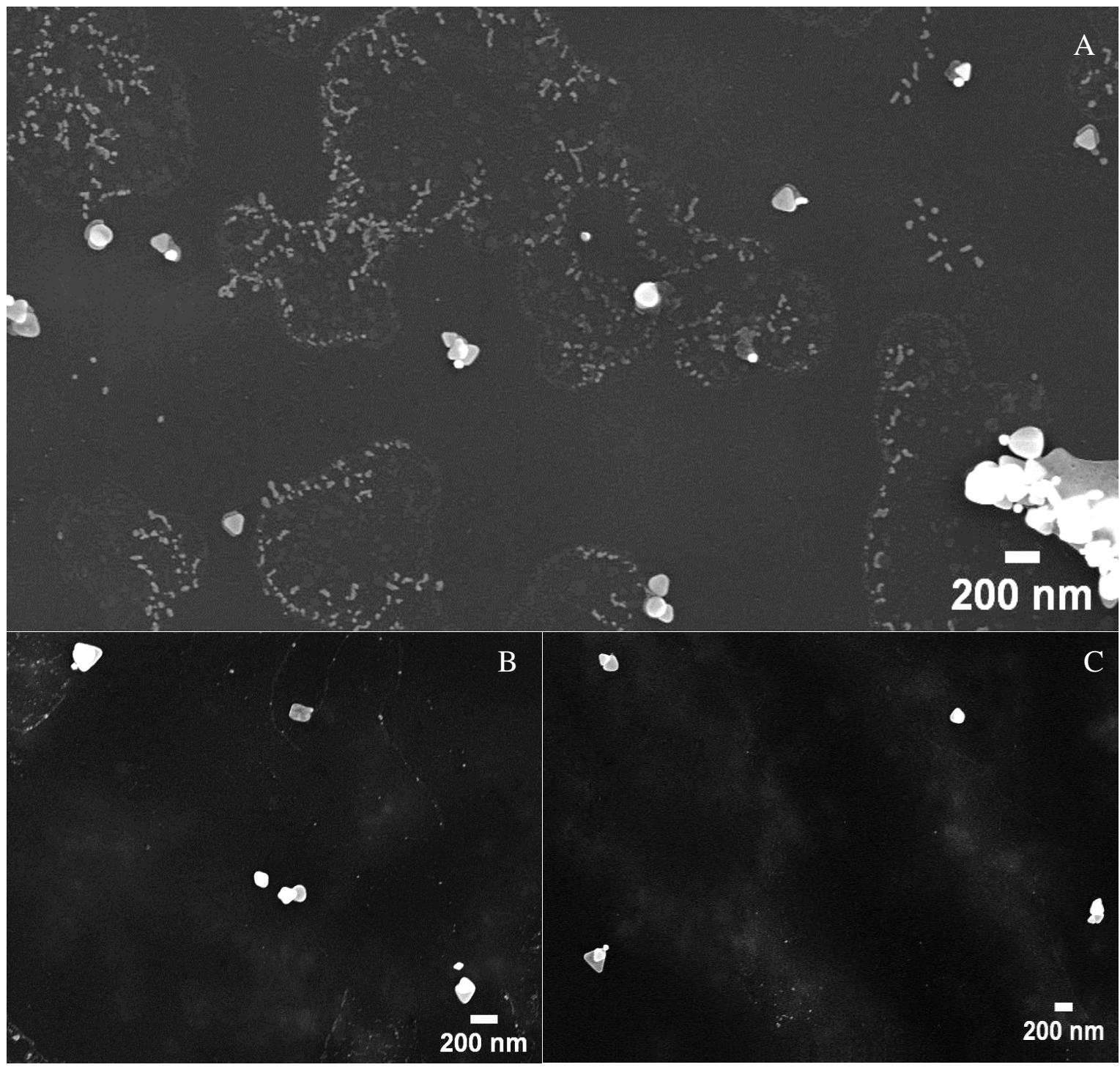

Figure 6.2: A), B) \& C) Representative SEM images of gold nanoprism dimers. 

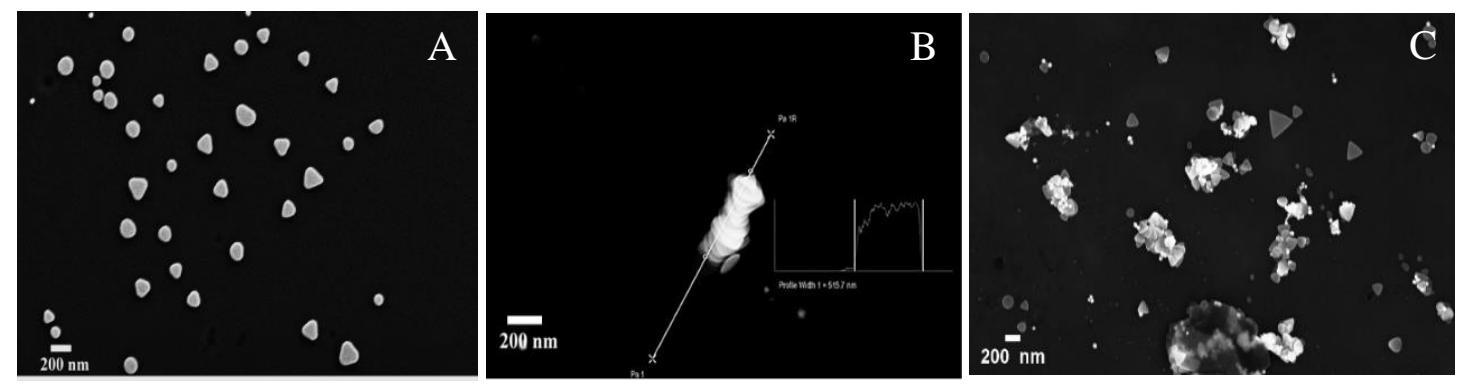

Figure 6.3: Representative SEM images of control experiments. A) PEG coated nanoprisms, B) face-to-face stacking of DNA-A and DNA-A" nanoprisms, C) cluster formation due to mixed PEG/DNA surface functionalization.

The lack of interaction for PEG coated nanoprisms and the face-to-face stacking of nanoprisms when the nanoprisms were densely and symmetrically functionalized with complementary DNAs indicates that dimers and trimers are formed through facet selective DNA interactions only. Additionally, the instance of cluster formation for mixed dual coating on the nanoprisms highlights the importance of efficient asymmetric functionalization of nanoprisms with either DNA or PEG on their two major facets for the formation of dimers and trimers.

While a good portion of nanoprism dimers possess a face-to-face orientation, there were also dimer populations with interstitial arrangement. This could be because of two reasons: first, the polydispersity of nanoprisms may cause uneven DNA-induced interactions between nanoprisms, and second, the presence of a mixture of PEG and DNA coatings, as compared to the presence of only either 
DNA or PEG coatings, on one of the major facets of some nanoprisms may have led to the formation of interstitial dimer configurations of nanoprisms.
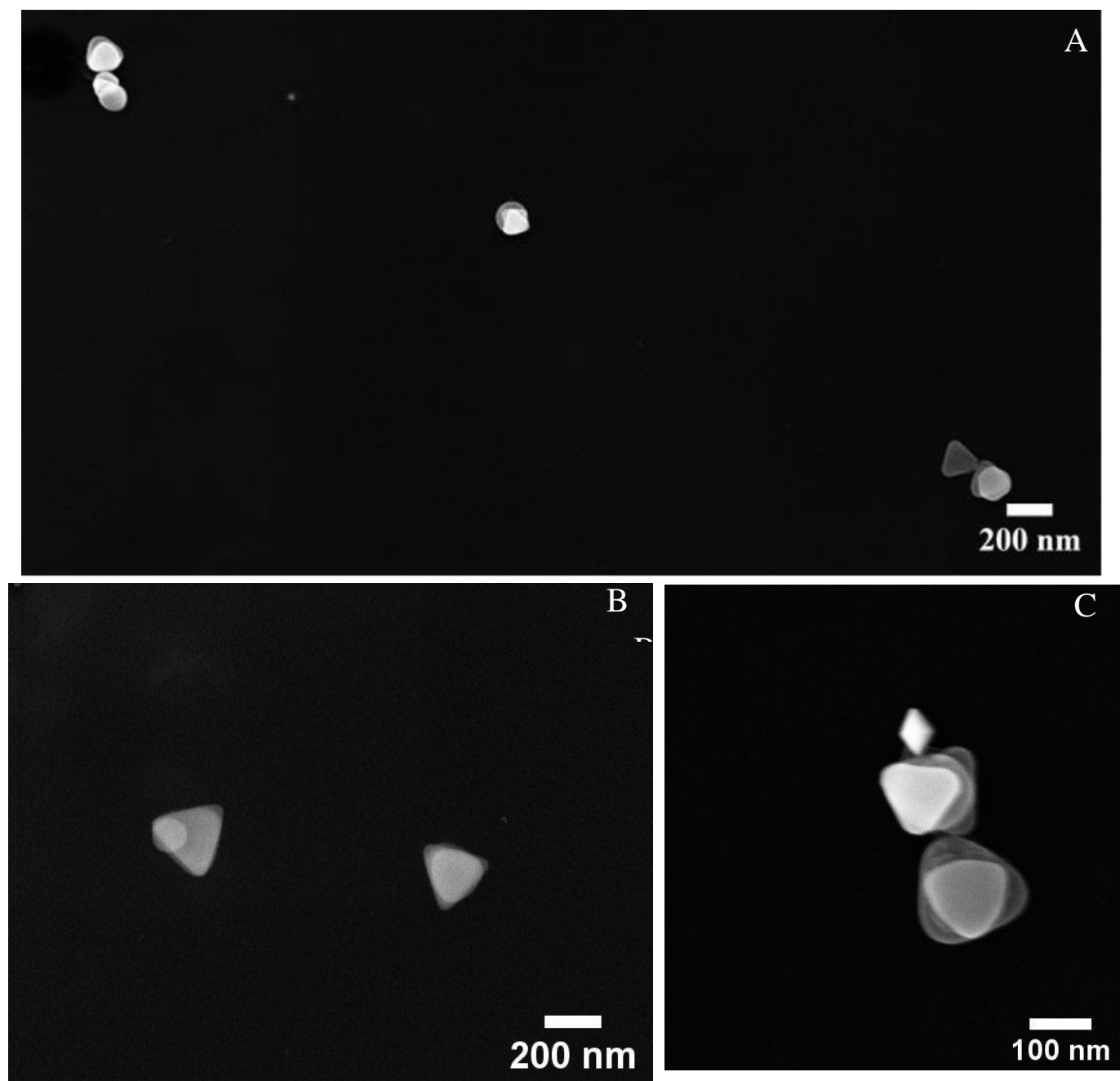

Figure 6.4: A), B) \&C) Representative SEM images of assembled nanoprism trimers.

The synthesis of trimers proceeds through DNA interaction between DNA-A" coated nanoprisms (DNA-A" | DNA-A" nanoprisms) and Janus PEG | DNA-A nanoprisms. To generate trimer structures, we mixed DNA coated nanoprisms with 
Janus nanoprisms in a 1: 2 molar ratios. Figure 6.4 shows the representative SEM images of assembled face-to-face nanoprism trimers. As illustrated in SEM images, nanoprisms are arranged in a face-to-face configuration to form trimer structures, which lie down in direction perpendicular to the substrates (glass slides or TEM grids). The yield of trimers was found to be around only $30-40 \%$, which is lower than that of dimers. The rest of the products in the assembly of nanoprisms trimers were singlets, dimers and clusters of nanoprisms. Similar to what was observed for the dimer assembly, there were both perfect face-to-face trimers and interstitial configurations of nanoprism interspersed among the trimer structures.

Figure 6.5 shows UV-vis absorption spectra for the formation dimers and trimers of nanoprisms. The surface plasmon resonance of nanoprisms redshifted and broadened after dimer and trimer assembly.
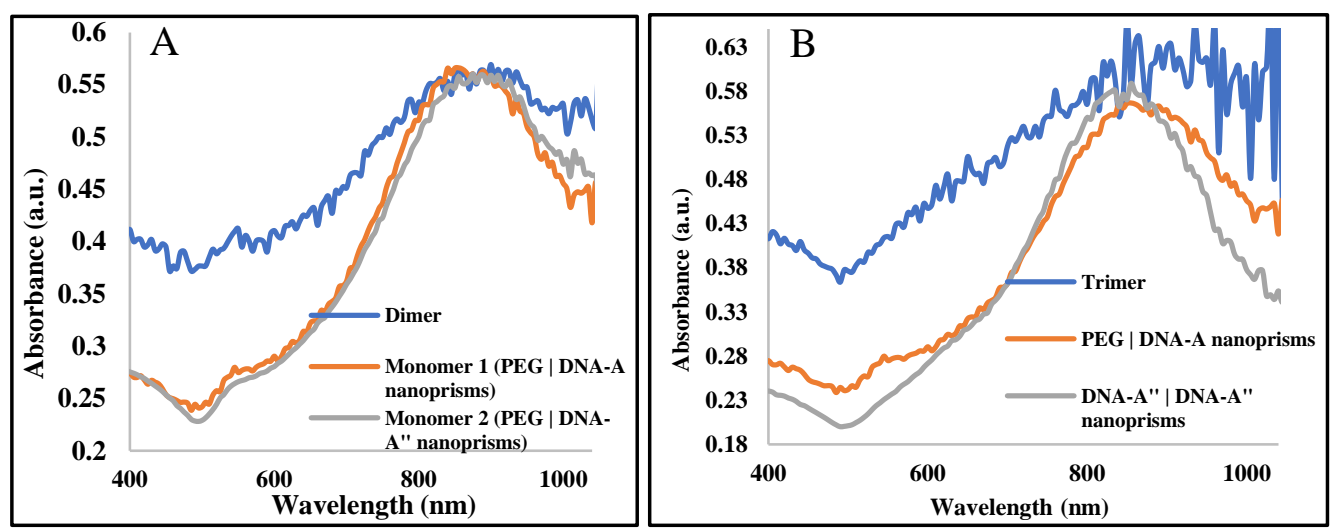

Figure 6.5: A) UV-Vis spectra of assembled gold nanoprism dimer, B) $\mathrm{UV}-\mathrm{Vis}$ spectra of assembled gold nanoprism trimer.

The broadening might be due to the formation of small clusters of nanoprisms. Additionally, the assembly of dimers and trimers induced $30 \mathrm{~nm}$ and $50 \mathrm{~nm}$ red 
shift in the SPR of the nanoprisms, respectively. The red shift is due to plasmonic coupling between neighboring nanoprisms in the dimer and trimer assemblies.

\subsubsection{Plasmonic properties of single nanoprim dimers and trimers}

Next, we studied the optical properties of nanoprism dimers and trimers at the single particle level using a combined approach of hyperspectral imaging and SEM. To correlate single nanoprism dimers and trimers with their plasmonic response, we located single dimers and trimers on a glass slide with etched numerical markers and collected their scattering spectra by hyperspectral imaging. Figure 6.6 shows representative SEM images of single gold nanoprism monomers, dimers and trimers as well as hyperspectral image of single gold nanoprism monomers, dimers and trimers marked with red rectangles and their corresponding scattering profiles. The scattering spectrum of monomer nanoprisms shows a single LSPR peak at 684 $\mathrm{nm}$. In contrast, the scattering spectrum for dimers and trimers exhibit two distinct LSPR modes, which are significantly red shifted compared to the LSPR of nanoprism monomers. The peaks at $850 \mathrm{~nm}$ and at $764 \mathrm{~nm}$ can be assigned as the low energy bonding dipole-quadrupole mode and high energy antibonding dipolequadrupole mode respectively. In addition, the trimer configuration shows weak higher order plasmon modes (see below). This could be due to the retardation or finite size effect as the quantum confinement or size dependence of dielectric coefficients becoming negligible. 

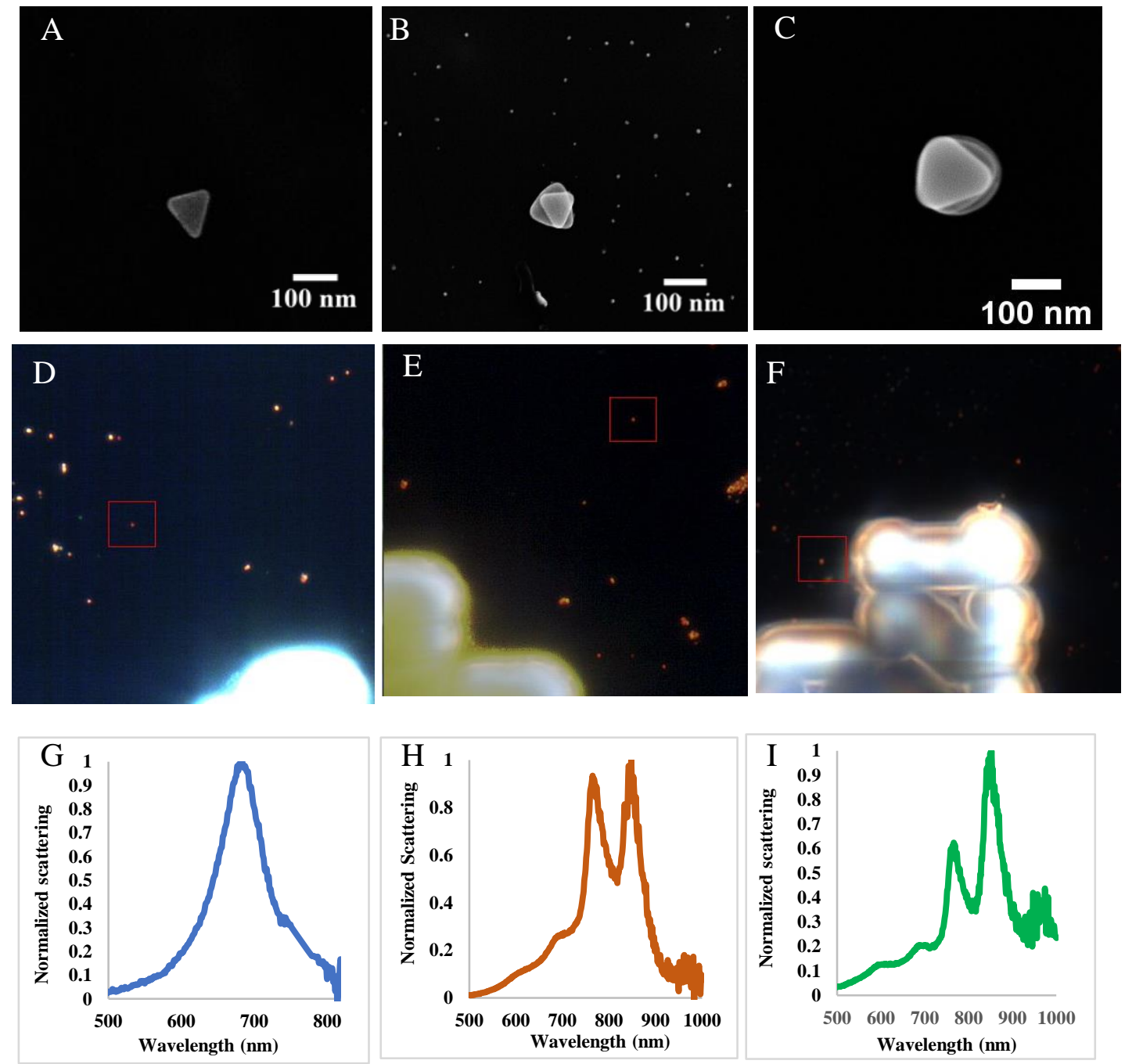

Figure 6.6: A), B) \& C) Representative SEM images of a single nanoprism, a nanoprism dimer and a nanoprisms trimer respectively; D) \& G) Hyperspectral image of nanoprisms on a marked glass slide and the scattering spectrum of the particle marked with a red rectangle, respectively; E) \& $\mathrm{H}$ ) Hyperspectral image of a nanoprism dimer on a glass slide and the scattering spectrum of the particle marked with red rectangle, respectively ; F) \& I) Hyperspectral image of a nanoprism trimer on a glass slide and the scattering spectrum of the particle marked with red rectangle, respectively. 
The peak at around $850 \mathrm{~nm}$ in the scattering profile for both dimer and trimer is known as a bright super-radiant mode resulting from the constructive interference of their radiated fields. ${ }^{294,314}$ More importantly, the scattering profile for both dimer and trimer reveal Fano type resonance with a characteristic narrow and asymmetric dip at around $817 \mathrm{~nm}$ known as a Fano minima. Fano resonance occurs through the interference between bright super-radiant and dark sub-radiant modes or coupling of bright super radiant mode to dark sub-radiant mode via near field interaction. ${ }^{315-317}$

Plasmonic nanostructures that exhibit strong Fano resonance can have a range of applications. ${ }^{300,318}$ For example, these nanostructures can be used in nanoscale waveguiding. The propagation of surface plasmon polaritons along a chain of $1 \mathrm{D}$ nanostructures at their Fano minimum can generate highly dispersive and relatively scatter-free waveguiding. These structures can also find application as optical cavities, because a large amount of energy can be stored in the dark mode. More importantly, these structures are ideal for nanoscale LSPR sensing applications because of their higher sensitivity to the changes in geometry or surrounding environment than the primitive modes of the nanostructure.

To be able to reliably use the scattering spectrum of dimers and trimers as a readout for sensing, homogeneity and reproducibility in the plasmonic response in terms of spectral pattern and spectral shift are essential. Figure 6.7 shows the scattering spectra of 6 different dimer and 6 trimer constructs. The spectral pattern for both dimer and trimer constructs are consistent. The spectra contain two major 
modes at the wavelength of around 764 and $850 \mathrm{~nm}$, which are red shifted by 80 $\mathrm{nm}$ and $164 \mathrm{~nm}$ respectively from the LSPS of the monomer nanoprism at $684 \mathrm{~nm}$. Additionally, the spectra exhibit a narrow and asymmetric Fano dip.
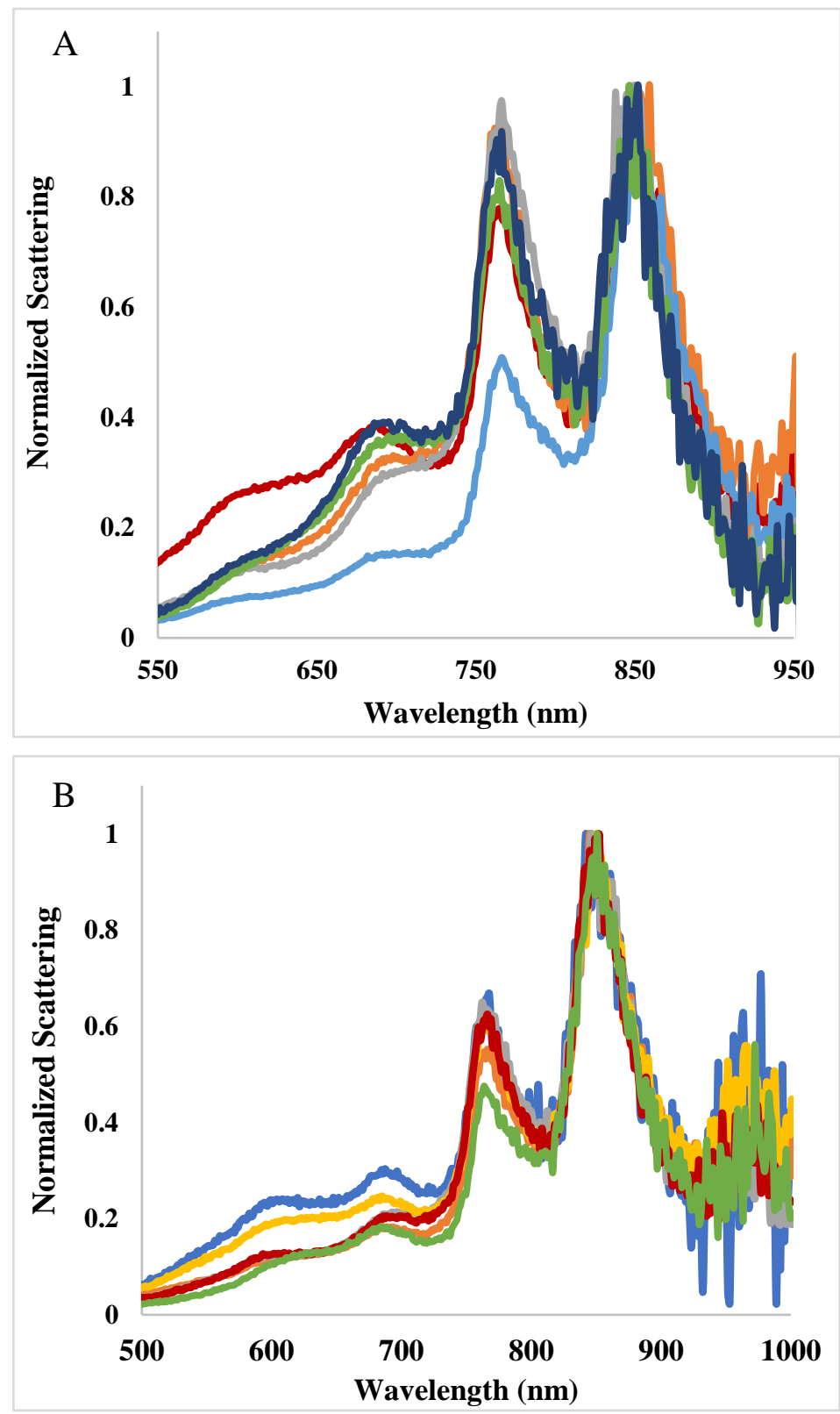

Figure 6.7: A) Scattering spectra of 6 single nanoprism dimers \& B) Scattering spectra of 6 single nanoprism trimers. 
These spectral shifts, which are dependent on the interparticle distance can be controlled by either changing the number of base pairs in the connecting DNA or solvent induced DNA bond contraction. Therefore, these dimers and trimers could potentially be used in plasmonic ruler applications.

\subsection{Conclusion}

In Summary, we demonstrated a DNA directed self-assembly approach for the synthesis of gold nanoprism dimers and trimers in solution. Asymmetric PEG-DNA dual functionalization of nanoprisms restricted their interaction to only DNA containing facets of the nanoprisms while PEG containing surfaces didn't interact, thereby affording the synthesis of plasmonic dimers and trimers. The plasmonic response of single dimers and trimers were studied using hyperspectral imaging combined with SEM. Both dimers and trimers exhibited reproducible spectral patterns and red shifts in LSPR, which are important for biochemical sensing based on an optical readout of the nano-constructs. Additionally, the scattering spectra of dimers and trimers showed a Fano dip at $817 \mathrm{~nm}$ induced by near field coupling of dark mode to bright mode. At the Fano minima, energy is stored in a dark mode. These findings highlight the potential of plasmonic nanoprism dimers and trimers for various

applications, including nanoscale waveguides, switching, optical imaging and sensing. 


\section{CHAPTER 7}

\section{CONCLUSION AND FUTURE DIRECTIONS}




\subsection{Summary and conclusions}

The work presented in this thesis focuses on the asymmetric surface functionalization and DNA guided assembly of nanoscale anisotropic building blocks for the fabrication of targeted nanostructures and their optical properties. A facile method was developed to controllably functionalize two major facets of anisotropic gold nanoprisms with distinct molecular coatings such as DNA and PEG/hexadecane to fabricate multifunctional Janus particles from an anisotropic core. While Janus particles have been known for quite a long time and various synthetic approaches have been reported for the synthesis of a diverse range of Janus particles, very little has been done on the synthesis of Janus particles from anisotropic cores. Two different anisotropic Janus gold nanoprisms, i.e. DNA | PEG and DNA | HexaD were synthesized by functionalizing one facet of the nanoprism with a thiolated DNA strand while the other facet was coated with thiolated hexadecane or PEG. DNA | HexaD Janus gold nanoprisms are amphiphilic and aligned themselves at the interface in a biphasic water/chloroform system and showed surface selective interaction to both hydrophilic and hydrophobic surfaces through the hydrophilic DNA containing facet and hydrophobic HexaD containing facet, respectively. Moreover, PEG | DNA Janus nanoprisms exhibited surface selective DNA-DNA interactions with spherical nanoparticles and asymmetric self-assembly through DNA containing facets.

Asymmetric functionalization of nanoprisms allowed the fabrication of targeted nanostructures such as dimers and trimers of gold nanoprisms. To synthesize dimers, two sets of Janus nanoprisms, PEG | DNA-A and PEG | DNA-A" were synthesized 
and mixed in a 1.1 molar ratio while nanoprisms trimer were synthesized by mixing Janus PEG | DNA-A with DNA-A" | DNA- $\mathbf{A}^{\prime \prime}$ in a 2:1 molar ratio. Optical properties of nanoprism dimers and trimers were studied at single particle level through hyperspectral imaging.

Moreover, we also explored the artificial directional interaction and shape effect of anisotropic nanoprisms to understand DNA-mediated hierarchical organization and crystallization of nanoprisms into 1D and 3D nanostructures. The dense layer of DNA ligand coatings drive the directional interactions between nanoparticles and leads to assembly with controlled symmetry. DNA-mediated interactions between complementary DNA strands anchored on the nanoprisms' flat surfaces led to the formation of $1 \mathrm{D}$ columnar stacks of nanoprisms. The average size of $1 \mathrm{D}$ crystals was around $500 \mathrm{~nm}-1.2 \mu \mathrm{m}$. Melting analysis showed sharp melting transitions with a characteristic melting point at $68.5^{\circ} \mathrm{C}$, indicating the dense DNA functionalization of nanoprisms.

The formation of 3D superlattices of nanoprisms was achieved though long-range interactions between 1D stacks that was established by introducing thermal energy. The simplest and most common thermal annealing method to generate nanoparticle superlattices is to heat and hold the initially formed nanoparticle aggregates $2-4^{\circ} \mathrm{C}$ below their melting transition point. When this thermal profile was applied and nanoprism $1 \mathrm{D}$ crystals were heated from $66^{\circ} \mathrm{C}$ to just below the $\mathrm{T}_{\mathrm{m}}$ of $1 \mathrm{D}$ stacks for several hours, 1D crystals gradually formed 3D superlattices with sizes ranging from 5-15 $\mu \mathrm{m}$ in diameter. High magnification SEM images showed that 1D crystals were 
assembled in both perpendicular and horizontal directions, resulting in highly polycrystalline nanoprism superlattices with crystal defects. The lack of highly ordered hexagonal and honeycomb nanoprism superlattices, as expected from selfassembly of plate and rod type nanoparticles, could be attributed to highly polydisperse nanoprisms. Prior work demonstrated that colloidal crystals formed using DNA tolerate only $10 \%$ or less polydispersity in nanoparticle core size for forming discrete geometric structures. Another important factor for the long-range ordering of nanoprisms was the concentration of nanoprism dispersions. Although dispersions of nanoprisms at low concentrations (below 2 OD) were able to generate 1D crystals, only a few 3D superlattices were formed for the same concentration of nanoprisms, even with extended annealing. A prior study has demonstrated that long range ordering of $1 \mathrm{D}$ stacks of triangular prisms occurs only when the concentration is 50 $\mu \mathrm{M} .{ }^{319}$ In this study, the initial concentration of the nanoprism dispersion was 2.4 OD for each complementary DNA pair for the formation of 1D stacks, which was then concentrated two fold before thermal annealing by spinning down using a centrifuge.

The applicability of 3D nanoprism superlattices as a plasmonic substrate was evaluated by measuring SERS and SEF enhancement of model compounds using 3D superlattices. The large number of closely arranged nanoprism arrays in the 3D crystal generated an ensemble of plasmonic hots spots, which gave rise to significant enhancement in SERS and SEF. The estimated SERS enhancement factor was $2.91 \times 10^{6}$ for the SERS analysis of methylene blue with a low detection limit of $10^{-10}$ M. We also assessed the reproducibility in the SERS signal using 3D superlattice by 
statistical analysis of both spot-to-spot and sample-to-sample variation. While the calculated co-efficient of variation $(\mathrm{CV})$ for sample-to-sample variation was only $5.6 \%$, indicating excellent reproducibility in the SERS performance of 3D superlattice, the spot-to-spot variation was as high as $28 \%$. This was due to larger size differences of superlattices, which resulted in varying degrees of collective plasmonic response. We then investigated the crystal size effect on the SERS response and found that the larger the size of superlattices, the higher the SERS intensities. SEF enhancement was evaluated by photoluminescence analysis of Alexa Fluor Phalloidin 647. A maximum of 5.5-fold increase in PL intensity of the dye was observed on the 3D superlattices.

Finally, we studied the factors that affect DNA mediated interactions between nanoscale anisotropic gold nanoprisms and microscale polymer beads and DNA induced crystallization of nanoprisms coated PS beads. DNA mediated interaction between nanoprisms and PS beads generated nanoprism-coated single beads, dimers of beads and bead clusters (3-7 beads). The effect of solution ionic strength on the loading density of nanoprisms was also investigated. While no clear trend in the salt effect on the loading density of nanoprisms onto PS beads was observed, DLS size measurements showed that the size of bead cluster increased with increasing solution ionic strength. Furthermore, the plasmon resonance band of nanoprisms increasingly red shifted with increasing salt concentration. This is because higher ionic strength sufficiently screens repulsion between negative changed DNA strands and allows greater interaction between nanoprism loaded beads. 
Fluorescence emission spectra of PS beads before and after nanoprism immobilization showed that the Fluorescence intensity of PS beads dropped by $78 \%$ due to the quenching effect of nanoprisms, which could be explained as damping of the PS beads' molecular dipole by the attached nanoprisms due to phase induced suppression of radiative decay rate of the fluorescent PS beads. Melting analysis of PS bead/nanprism conjugates showed sharp melting transitions with the characteristic melting point at $78^{\circ} \mathrm{C}$, while control experiments didn't show any such thermal profile. This indicates the dense DNA functionalization of nanoprisms and PS beads and that de-hybridization of DNA stands anchored on the nanoprisms and PS beads resulted in the sharp melting transition.

Thermal annealing of PS beads/nanoprisms conjugates below $\mathrm{T}_{\mathrm{m}}$ followed by slow cooling facilitated long range interaction between nanoprism-coated beads, which led to the formation of 3D PS bead/nanoprism aggregates. The average aggregate size was 10-20 $\mu \mathrm{m}$, consisting of 20-50 PS beads. The applicability of 3D nanoprism/PS bead aggregates as a SERS substrate was investigated by the SERS measurement of a model compound, methylene blue, using the 3D substrate. While the Raman spectrum of MB on PS bead aggregates is masked by the autofluorescence of the PS beads, such fluorescence background was not observed for the 3D PS beads/nanoprisms aggregates and 3D PS beads/nanoprisms aggregates due to quenching of the PS beads' fluorescence by the nanoprisms.

The presence of a large number plasmonic hot spots in the laser focal volume resulted in high SERS enhancement $\left(<10^{5}\right.$ ) with a low MB detection limit of $10^{-10} \mathrm{M}$. 
Analysis of spot-to-spot variation in the SERS signal showed that the average signal intensity at $1621 \mathrm{~cm}^{-1}$ for the PS bead/nanoprism aggregates was 3580.4 counts with a coefficient of variation (CV) of only $18 \%$, indicating good reproducibility of the 3D PS bead/nanoprism aggregates as a SERS substrate.

\subsection{Future Directions}

Looking ahead to future work, the results and findings presented in this dissertation would enable numerous potential research investigations. In regard to Janus particle synthesis, the method we described here will be not only transferable to other isotropic and anisotropic nanoparticles, but also applied to other biomolecules such as proteins and peptides, and other polymeric coatings such as chitosan, dendrimers, poly(allylamine) and poly(styrenesulfonate) as surface coatings, which would allow the fabrication of more complex Janus particles. Moreover, initial cell studies showed that our DNA-coated nanoprisms are well internalized by the glioblastoma cells (Figure 7.1). It will be intriguing to study the difference in the cellular uptake of just DNA coated nanoprisms and Janus nanoprisms. Prior work demonstrated that the presence and spatial segregation of hydrophobicity and charges on Janus particle surfaces induces nanoparticles attachment more strongly to lipid bilayers and causes defects more effectively than nanoparticles with uniform surface coatings. ${ }^{320}$ 

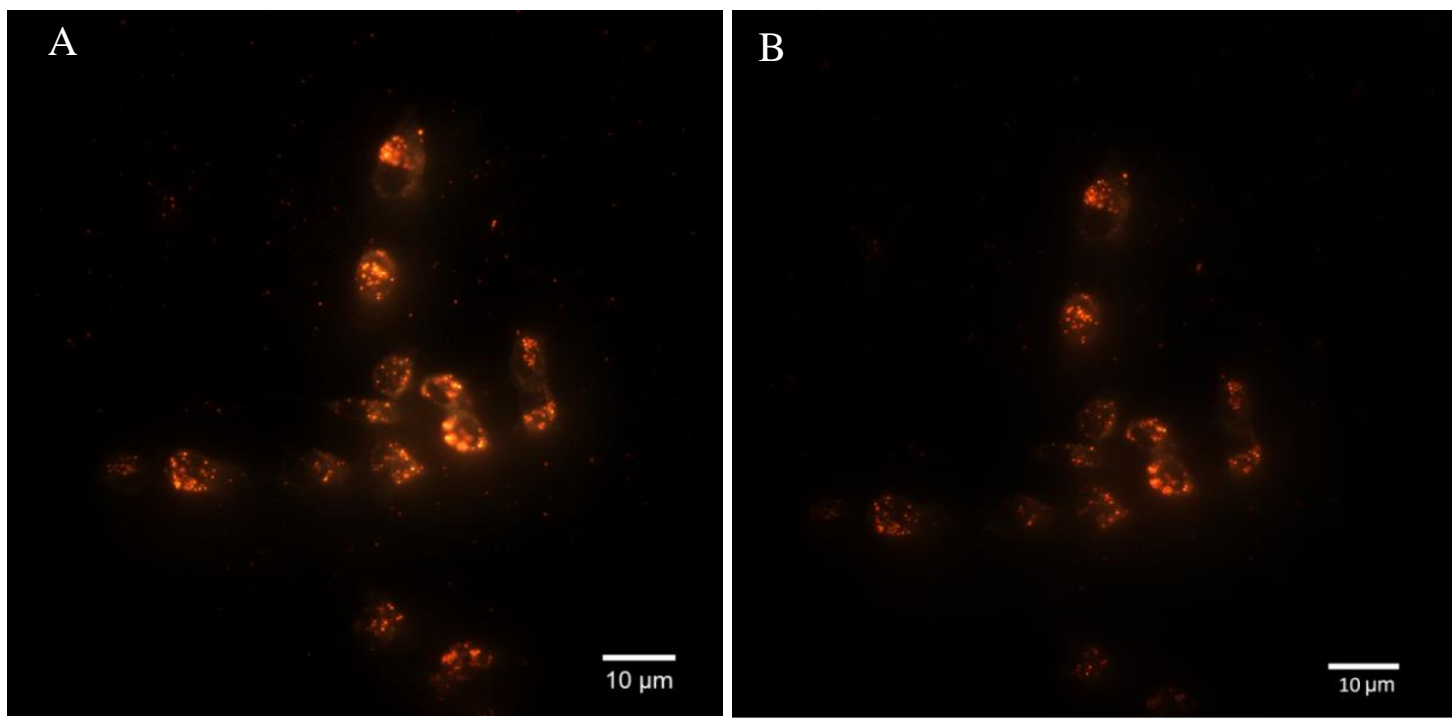

Figure 7.1: A) \& B) Dark field microscopy images of cellular internalization of DNA coated gold nanoprisms.

One of the major disadvantages of using a glass slides as a substrate for protecting part of nanoprisms for controllable dual coating was the low yield of Janus particle due to low surface area of glass slide. In addition, it's cumbersome to handle glass slides. In order to increase the yield, we utilized the high surface area of glass wool and glass beads. Preliminary results show that the use of glass wool (1g) and glass beads $(10 \mathrm{~g})$, whose surface area is 3 orders of magnitude larger than that of 10 glass slides (Table 7.1), as a template dramatically increases the particle yield. 
Table 7.1: Comparison of surface areas of different glass substrates

\begin{tabular}{ccc}
\hline Materials & Amount & Surface area \\
\hline Glass slide & 10 units & $1.8 \times 10^{14}$ \\
Glass beads & $10 \mathrm{~g}$ & $1.6 \times 10^{17}$ \\
Glass wools & $1 \mathrm{~g}$ & $1.8 \times 10^{17}$ \\
\hline
\end{tabular}

Figure 7.2 shows the representative SEM images of glass wools, monolayer of nannoprisms on APTES functionalized glass wools and glass wools after lifting nanoprisms off the surface and Figure $\mathbf{7 . 3}$ shows the representative SEM images of glass beads, monolayer of nannoprisms on APTES functionalized glass beads and glass beads after lifting nanoprisms off the bead surface. While 10 glass slides generate only $1 \mathrm{~mL}$ of $0.5-1.0$ OD Janus nanoprisms, both $1 \mathrm{~g}$ glass wool and $10 \mathrm{~g}$ glass beads produces 3-5 $\mathrm{mL}$ of 6-10 OD of Janus nanoprisms. However, it requires extra purification steps after lift-off from these surfaces. Future work could optimize the use of glass wool and glass beads instead of glass slides and focus on the mitigation of the need of extra purification steps. 


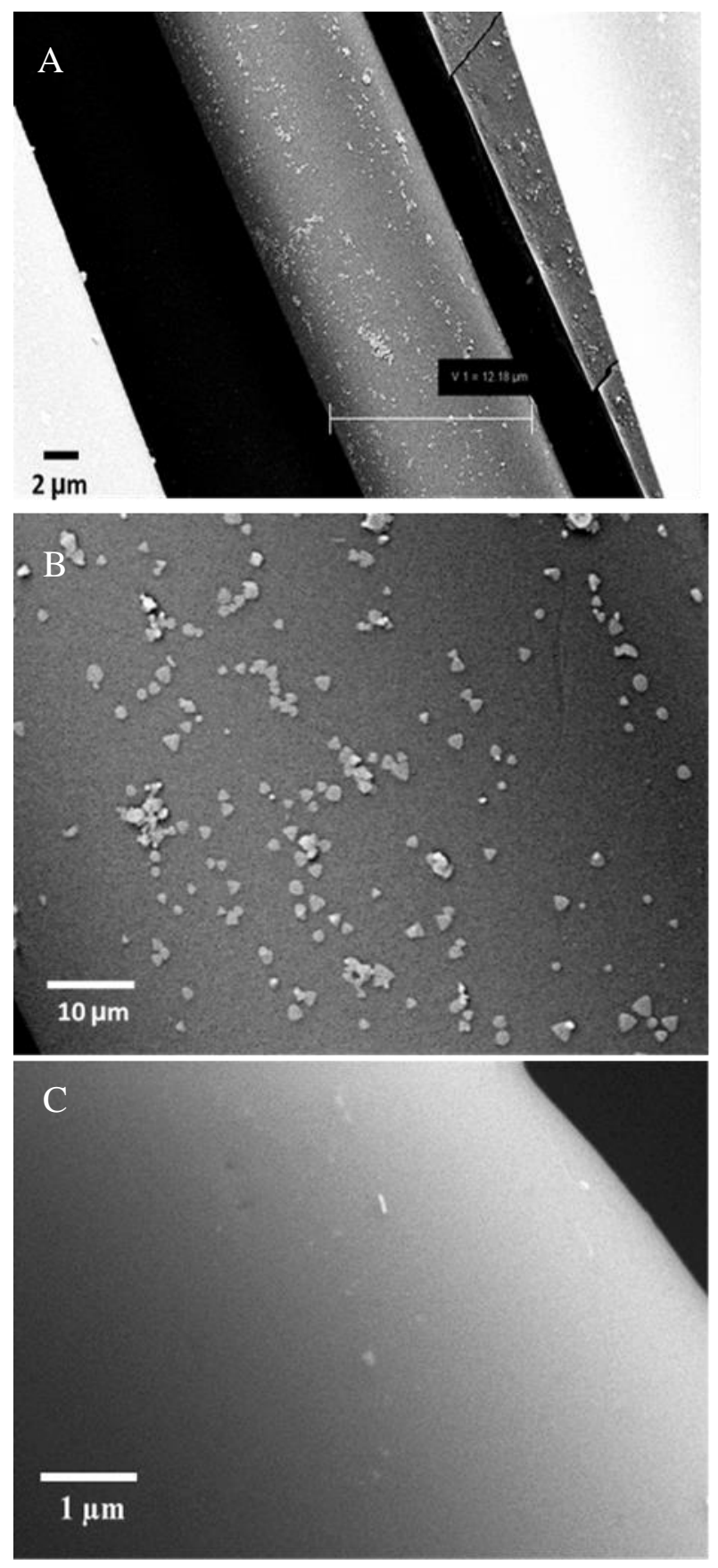

Figure 7.2: Representative SEM images of A) \& B) monolayers of nanoprisms on APTES functionalized glass wools and C) glass wool after the removal of nanoprisms. 

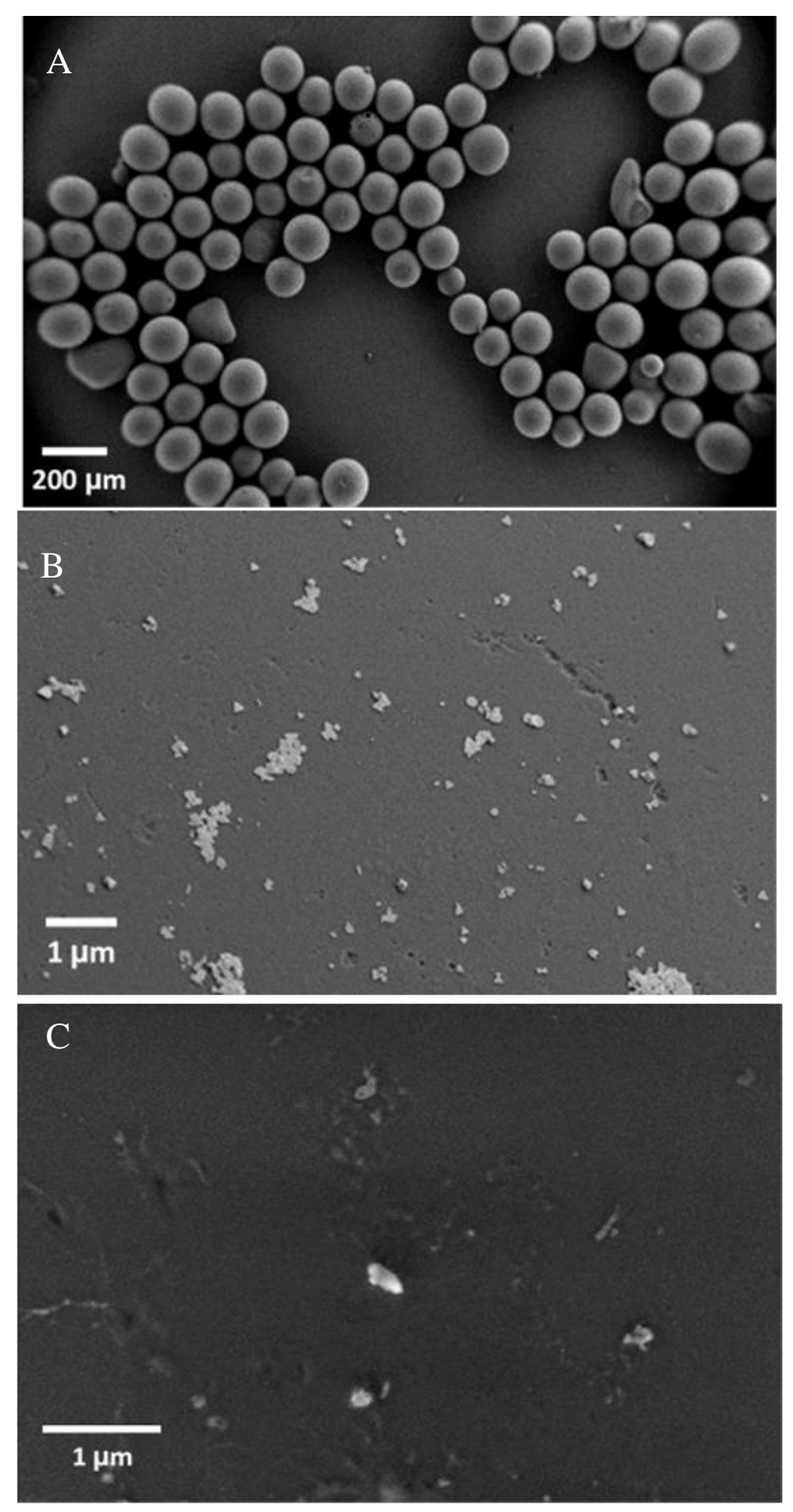

Figure 7.3: Representative SEM images of A) glass beads \& B) monolayer of nanoprisms on APTES functionalized glass beads and C) a glass bead surface after removal of nanoprisms. 
With respect to nanoprism dimers and trimers, an innovative drug carrier could be developed. For instance, replacing the inside DNA coating of dimers/trimers with a hydrophobic coating would allow the fabrication of a hydrophobic drug carriers, where light-responsive drugs could be loaded to interparticle spaces of dimers and trimers. Moreover, light scattering of single nanoprism dimers and trimers showed distinct plasmonic shift (80-160nm) and spectral patterns, which could find applications in highly sensitive biosensing.

One of the primary goals of this dissertation was to study the kinetics of nanoprism face-to-face assembly and control the length of nanoprism 1D stacks by capping the growth with the addition of Janus nanoprisms. While these were not accomplished in this thesis, the findings pave the way to study the kinetics and control the length of 1D stacks. Typically, particle assembly using DNA happens significantly faster than the process of particle crystallization, which makes the control of the length of nanoprisms stacks and study the assembly kinetics impossible. However, the DNA strands used in this study requires heating followed by cooling for the face-to-face assembly and the melting analysis shows the time window (Figure 7.4), when particle assembly occurs. By adding Janus nanoprisms at different time points within the time window of assembly, the length of nanoprisms stack could be controlled. Knowing the time window for nanoprism assembly would also facilitate the kinetic study of face-toface assembly and 1D crystal growth. 


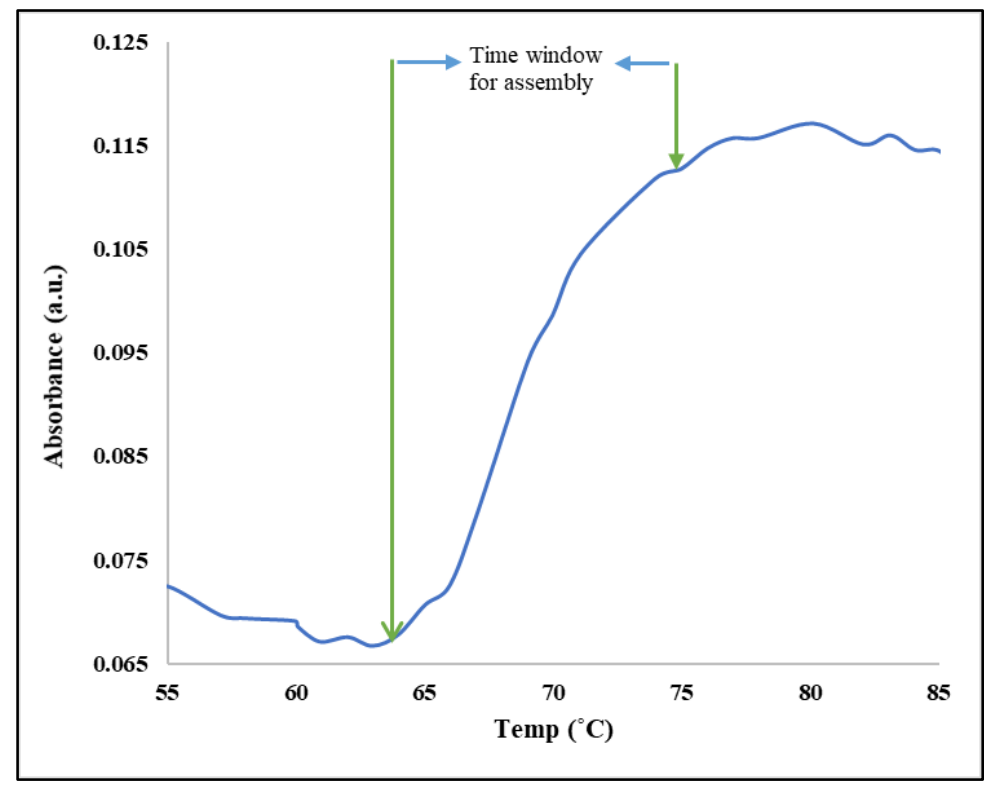

Figure 7.4: Time window for face-to-face assembly of nanoprisms.

Furthermore, due to high confinement of electromagnetic energy, propagating surface plasmon polaritons along 1D nanostructures provides one of the best potential ways to construct next-generation circuits that can overcome the speed limit of electronics by using light. Therefore, the properties of propagating surface plasmon polaritons (SPPs) along 1D nanoprism stacks could find applications as nanophotonic circuits and nano-plasmonic waveguide circuits.

Regarding PS beads/nanoprisms conjugates, while we have been able to obtain a dense monolayer of nanoprisms on PS bead surfaces in this study, the consistency in uniform loading of nanoprisms onto PS beads was still lacking. Future work can fix the inconsistency by controlling the cooling rate during annealing to provide enough 
time window for consistently high-density coating of nanoprisms on the PS beads. While the majority of previous studies on polymer-nanoparticle conjugates was based on the loading of spherical nanoparticles onto polymer beads, this work documents the assembly of anisotropic nanoprisms. Future work could focus on the loading of other anisotropic shaped particles (such as rods, cuboids, rhomboids) or combinations of two or three different nanoparticles, which would allow the fabrication of more complex polymer-nanoparticle conjugates for SERS and nano-photonics.

The fluorescence quenching of PS beads by nanoprisms could be corelated to the concentration of DNA, which would facilitate the design and development of a fluorescence quenching based assay. 


\section{REFERENCES}

1) Zhang, S.; Pelligra, C. I.; Feng, X.; Osuji, C. O. Adv. Mater. 2018, 30 (18), 1705794.

2) Khan, I.; Saeed, K.; Khan, I. Arab. J. Chem. 2019, 12 (7), 908-931.

3) Yeh, Y.-C.; Creran, B.; Rotello, V. M. Nanoscale 2012, 4 (6), 1871-1880.

4) Kim, I. T.; Nunnery, G. A.; Jacob, K.; Schwartz, J.; Liu, X.; Tannenbaum, R. J. Phys. Chem. C 2010, 114 (15), 6944-6951.

5) Grzelczak, M.; Vermant, J.; Furst, E. M.; Liz-Marzán, L. M. ACS Nano 2010, 4 (7), 3591-3605.

6) Toma, H. E. Curr. Sci. 2008, 95 (9), 1202-1225.

7) Fendt, L.-A.; Stöhr, M.; Wintjes, N.; Enache, M.; Jung, T. A.; Diederich, F. Chem. Eur. J. 2009, 15 (42), 11139-11150.

8) Lele, P. P.; Mittal, M.; Furst, E. M. Langmuir 2008, 24 (22), 12842-12848.

9) Lee, S. H.; Liddell, C. M. Small 2009, 5 (17), 1957-1962.

10) Roy, D.; Mondal, D.; Goswami, D. Sci. Rep. 2016, 6 (1), 23318.

11) Dong, Z.; Asbahi, M.; Lin, J.; Zhu, D.; Wang, Y. M.; Hippalgaonkar, K.; Chu, H.-S.; Goh, W. P.; Wang, F.; Huang, Z.; Yang, J. K. W. Nano Lett. 2015, 15 (9), 5976-5981.

12) Booth, S. G.; Dryfe, R. A. W. J. Phys. Chem. C 2015, 119 (41), 23295-23309.

13) Shereda, L. T.; Larson, R. G.; Solomon, M. J. Phys. Rev. Lett. 2008, 101 (3), 038301.

14) Jones, M. R.; Seeman, N. C.; Mirkin, C. A. Science 2015, 347 (6224), 1260901.

15) Mirkin, C. A.; Letsinger, R. L.; Mucic, R. C.; Storhoff, J. J. Nature 1996, 382 (6592), 607-609.

16) Mondal, S.; Gazit, E. ChemNanoMat 2016, 2 (5), 323-332.

17) Wang, Y.; Xu, H.; Zhang, X. Adv. Mater. 2009, 21 (28), 2849-2864. 
18) Fendt, L. A.; Stohr, M.; Wintjes, N.; Enache, M.; Jung, T. A.; Diederich, F. Chem.Eur. J. 2009, 15 (42), 11139-11150.

19) Daniel, M. C.; Astruc, D. Chem. Rev. 2004, 104 (1), 293-346.

20) Mejia-Ariza, R.; Graña-Suárez, L.; Verboom, W.; Huskens, J. J. Mat. Chem. B 2017, 5 (1), 36-52.

21) Oh, J. H.; Park, D. H.; Joo, J. H.; Lee, J. S. Anal. Bioanal. Chem. 2015, 407 (29), 8627-45.

22) Sedlmeier, A.; Gorris, H. H. Chem. Soc. Rev. 2015, 44 (6), 1526-1560.

23) Hu, J.; Zhou, S.; Sun, Y.; Fang, X.; Wu, L. Chem. Soc. Rev. 2012, 41 (11), 43564378.

24) Jones, M. R.; Macfarlane, R. J.; Prigodich, A. E.; Patel, P. C.; Mirkin, C. A. J. Am. Chem. Soc. 2011, 133 (46), 18865-9.

25) Burrows, N. D.; Vartanian, A. M.; Abadeer, N. S.; Grzincic, E. M.; Jacob, L. M.; Lin, W. N.; Li, J.; Dennison, J. M.; Hinman, J. G.; Murphy, C. J. J. Phys. Chem. Lett. 2016, 7 (4), 632-641.

26) Jiang, X.; Liu, R.; Tang, P.; Li, W.; Zhong, H.; Zhou, Z.; Zhou, J. RSC Adv. 2015, 5 (98), 80709-80718.

27) Brann, T.; Patel, D.; Chauhan, R.; James, K. T.; Bates, P. J.; Malik, M. T.; Keynton, R. S.; O’Toole, M. G. J. Nanomater. 2016, 2016, 2036029.

28) Pattani, V. P.; Tunnell, J. W. Lasers Surg. Med. 2012, 44 (8), 675-84.

29) Jones, M. R.; Macfarlane, R. J.; Lee, B.; Zhang, J.; Young, K. L.; Senesi, A. J.; Mirkin, C. A. Nat. Mater. 2010, 9 (11), 913-7.

30) Glotzer, S. C. Nature 2012, 481 (7382), 450-2.

31) Zhou, Y.; Zhou, X.; Park, D. J.; Torabi, K.; Brown, K. A.; Jones, M. R.; Zhang, C.; Schatz, G. C.; Mirkin, C. A. Nano Lett. 2014, 14 (4), 2157-2161.

32) Millstone, J. E.; Hurst, S. J.; Metraux, G. S.; Cutler, J. I.; Mirkin, C. A. Small 2009, 5 (6), 646-64.

33) Reguera, J.; Langer, J.; Jiménez de Aberasturi, D.; Liz-Marzán, L. M. Chem. Soc. Rev. 2017, 46 (13), 3866-3885.

34) Sajanlal, P. R.; Sreeprasad, T. S.; Samal, A. K.; Pradeep, T. Nano Rev. 2011, 2. 
35) Millstone, J. E.; Park, S.; Shuford, K. L.; Qin, L.; Schatz, G. C.; Mirkin, C. A. J. Am. Chem. Soc. 2005, 127 (15), 5312-5313.

36) Yánez-Sedeño, P.; Campuzano, S.; Pingarrón, J. M. Appl. Mater. Today 2017, 9 , 276-288.

37) Xue, C.; Métraux, G. S.; Millstone, J. E.; Mirkin, C. A. J. Am. Chem. Soc. 2008, 130 (26), 8337-8344.

38) Jones, M. R.; Mirkin, C. A. Angew. Chem. Int. Ed. Engl. 2013, 52 (10), 2886-91.

39) Vutukuri, H. R.; Badaire, S.; de Winter, D. A.; Imhof, A.; van Blaaderen, A. Nano Lett. 2015, 15 (8), 5617-23.

40) Paramasivam, G.; Kayambu, N.; Rabel, A. M.; Sundramoorthy, A. K.;

Sundaramurthy, A. Acta Biomater. 2017, 49, 45-65.

41) Li, C. X.; Bolisetty, S.; Mezzenga, R. Adv. Mater. 2013, 25 (27), 3694-3700.

42) Milligan, W. O.; Morriss, R. H. J. Am. Chem. Soc. 1964, 86 (17), 3461-3467.

43) Chen, L.; Ji, F.; Xu, Y.; He, L.; Mi, Y.; Bao, F.; Sun, B.; Zhang, X.; Zhang, Q. Nano Lett. 2014, 14 (12), 7201-7206.

44) Ha, T. H.; Koo, H.-J.; Chung, B. H. J. Phys. Chem. C 2007, 111 (3), 1123-1130.

45) Malikova, N.; Pastoriza-Santos, I.; Schierhorn, M.; Kotov, N. A.; Liz-Marzán, L. M. Langmuir 2002, 18 (9), 3694-3697.

46) Shaik, F.; Zhang, W.; Niu, W. J. Phys. Chem. C 2017, 121 (17), 9572-9578.

47) Shankar, S. S.; Rai, A.; Ankamwar, B.; Singh, A.; Ahmad, A.; Sastry, M. Nat. Mater. 2004, 3 (7), 482-488.

48) Zhu, J.; Jin, X. L. Superlattices Microstruct. 2007, 41 (4), 271-276.

49) Zhai, Y.; DuChene, J. S.; Wang, Y. C.; Qiu, J.; Johnston-Peck, A. C.; You, B.; Guo, W.; DiCiaccio, B.; Qian, K.; Zhao, E. W.; Ooi, F.; Hu, D.; Su, D.; Stach, E. A.; Zhu, Z.; Wei, W. D. Nat. Mater. 2016, 15 (8), 889-95.

50) Zhang, G.; Jasinski, J. B.; Howell, J. L.; Patel, D.; Stephens, D. P.; Gobin, A. M. Nanoscale Res. Lett. 2012, 7 (1), 337.

51) Saverot, S.-E.; Reese, L. M.; Cimini, D.; Vikesland, P. J.; Bickford, L. R. Nanoscale Res. Lett. 2015, 10 (1), 241.

52) James, K. T.; O'Toole, M. G.; Patel, D. N.; Zhang, G.; Gobin, A. M.; Keynton, R. S. RSC Adv. 2015, 5 (17), 12498-12505. 
53) Poggi, E.; Gohy, J.-F. Colloid Polym. Sci. 2017, 295 (11), 2083-2108.

54) Walther, A.; Müller, A. H. E. Chem. Rev. 2013, 113 (7), 5194-5261.

55) Perro, A.; Reculusa, S.; Ravaine, S.; Bourgeat-Lami, E.; Duguet, E. J. Mater. Chem. 2005, 15 (35-36), 3745-3760.

56) Zhang, J.; Grzybowski, B. A.; Granick, S. Langmuir 2017, 33 (28), 6964-6977.

57) Lattuada, M.; Hatton, T. A. Nano Today 2011, 6 (3), 286-308.

58) Choi, S. Q.; Jang, S. G.; Pascall, A. J.; Dimitriou, M. D.; Kang, T.; Hawker, C. J.; Squires, T. M. Adv. Mater. 2011, 23 (20), 2348-2352.

59) Fan, X.; Yang, J.; Loh, X. J.; Li, Z. Macromol. Rapid. Commun. 2019, 40 (5), e1800203.

60) Cho, I.; Lee, K.-W. J. Appl. Polym. Sci. 1985, 30 (5), 1903-1926.

61) Archer, R. J.; Campbell, A. I.; Ebbens, S. J. Soft Matter 2015, 11 (34), 6872-6880.

62) Wei, D.; Ge, L. L.; Lu, S. H.; Li, J. J.; Guo, R. Langmuir 2017, 33 (23), 5819-5828.

63) Zenerino, A.; Peyratout, C.; Aimable, A. J. Colloid Interface Sci. 2015, 450, $174-$ 181.

64) Li, Z. F.; Lee, D. Y.; Rubner, M. F.; Cohen, R. E. Macromolecules 2005, 38 (19), 7876-7879.

65) Zhang, C.; Chang, M.-W.; Li, Y.; Qi, Y.; Wu, J.; Ahmad, Z.; Li, J.-S. RSC Adv. 2016, 6 (81), 77174-77178.

66) Nisisako, T.; Torii, T.; Takahashi, T.; Takizawa, Y. Adv. Mater. 2006, 18 (9), 11521156.

67) Zhao, Y.; Gu, H.; Xie, Z.; Shum, H. C.; Wang, B.; Gu, Z. J. Am. Chem. Soc. 2013, $135(1), 54-57$.

68) Love, J. C.; Gates, B. D.; Wolfe, D. B.; Paul, K. E.; Whitesides, G. M. Nano Lett. 2002, 2 (8), 891-894.

69) Zhang, L.; Yu, J.; Yang, M.; Xie, Q.; Peng, H.; Liu, Z. Nat. Commun. 2013, 4 (1), 1443.

70) Paunov, V. N.; Cayre, O. J. Adv. Mater. 2004, 16 (9-10), 788-791.

71) McConnell, M. D.; Kraeutler, M. J.; Yang, S.; Composto, R. J. Nano Lett. 2010, 10 (2), 603-609. 
72) Perro, A.; Meunier, F.; Schmitt, V.; Ravaine, S. Colloids Surf. A Physicochem. Eng. Asp. 2009, 332 (1), 57-62.

73) Delcea, M.; Madaboosi, N.; Yashchenok, A. M.; Subedi, P.; Volodkin, D. V.; De Geest, B. G.; Mohwald, H.; Skirtach, A. G. Chem. Commun. (Cambridge, U. K.) 2011, 47 (7), 2098-100.

74) Lin, C. C.; Liao, C. W.; Chao, Y. C.; Kuo, C. ACS Appl. Mater. Interfaces 2010, 2 (11), 3185-91.

75) Cayre, O.; Paunov, V. N.; Velev, O. D. Chem. Commun. 2003, (18), 2296-2297.

76) Anderson, K. D.; Luo, M.; Jakubiak, R.; Naik, R. R.; Bunning, T. J.; Tsukruk, V. V. Chem. Mater. 2010, 22 (10), 3259-3264.

77) Kim, S.-H.; Lee, S. Y.; Yang, S.-M. Angew. Chem. Int. Ed. Engl. 2010, 49 (14), 2535-2538.

78) Kocak, G.; Tuncer, C.; Bütün, V. Polym. Chem. 2017, 8 (1), 144-176.

79) Pawar, A. B.; Kretzschmar, I. Langmuir 2008, 24 (2), 355-358.

80) Liu, L.; Ren, M.; Yang, W. Langmuir 2009, 25 (18), 11048-11053.

81) Cha, B. G.; Piao, Y.; Kim, J. Mater. Res. Bull. 2015, 70, 424-429.

82) Wang, B.; Li, B.; Zhao, B.; Li, C. Y. J. Am. Chem. Soc. 2008, 130 (35), 1159411595 .

83) Lim, J. K.; Ciszek, J. W.; Huo, F.; Jang, J.-W.; Hwang, S.; Mirkin, C. A. Nano Lett. 2008, 8 (12), 4441-4445.

84) Wang, J.-Y.; Wang, Y.; Sheiko, S. S.; Betts, D. E.; DeSimone, J. M. J. Am. Chem. Soc. 2012, 134 (13), 5801-5806.

85) Walther, A.; Muller, A. H. E. Chemical Reviews 2013, 113 (7), 5194-5261.

86) Tian, L.; Zhang, B.; Li, W.; Li, X.; Fan, X.; Jia, X.; Zhang, H.; Zhang, Q. RSC Adv. 2014, 4 (52), 27152-27158.

87) Saito, N.; Nakatsuru, R.; Kagari, Y.; Okubo, M. Langmuir 2007, 23 (23), 11506-12.

88) Hu, S.-H.; Gao, X. J. Am. Chem. Soc. 2010, 132 (21), 7234-7237.

89) Nisisako, T. Curr. Opin. Colloid Interface Sci. 2016, 25, 1-12.

90) Lone, S.; Cheong, I. W. RSC Adv. 2014, 4 (26), 13322-13333. 
91) Chen, C.-H.; Shah, R. K.; Abate, A. R.; Weitz, D. A. Langmuir 2009, 25 (8), 43204323.

92) Xie, J.; Jiang, J.; Davoodi, P.; Srinivasan, M. P.; Wang, C. H. Chem. Eng. Sci. 2015, $125,32-57$.

93) Pochan, D. J.; Zhu, J.; Zhang, K.; Wooley, K. L.; Miesch, C.; Emrick, T. Soft Matter 2011, 7 (6), 2500-2506.

94) Du, J.; Armes, S. P. Soft Matter 2010, 6 (19), 4851-4857.

95) Rupar, P. A.; Chabanne, L.; Winnik, M. A.; Manners, I. Science 2012, 337 (6094), 559-62.

96) Erhardt, R.; Böker, A.; Zettl, H.; Kaya, H.; Pyckhout-Hintzen, W.; Krausch, G.; Abetz, V.; Müller, A. H. E. Macromolecules 2001, 34 (4), 1069-1075.

97) Wolf, A.; Walther, A.; Müller, A. H. E. Macromolecules 2011, 44 (23), 9221-9229.

98) Hiekkataipale, P.; Löbling, T. I.; Poutanen, M.; Priimagi, A.; Abetz, V.; Ikkala, O.; Gröschel, A. H. Polymer 2016, 107, 456-465.

99) Girard, M.; Millan, J. A.; de la Cruz, M. O., DNA-Driven Assembly: From Polyhedral Nanoparticles to Proteins. In Annu. Rev. Mater. Res., Vol 47, Clarke, D. R., Ed. 2017; Vol. 47, pp 33-49.

100) Ou, J.; Tan, H.; Chen, X.; Chen, Z. Nanomaterials 2018, 8 (12).

101) Heuer-Jungemann, A.; Liedl, T. Trends Chem. 2019, 1 (9), 799-814.

102) Fu, T. J.; Seeman, N. C. Biochemistry 1993, 32 (13), 3211-20.

103) He, Y.; Tian, Y.; Ribbe, A. E.; Mao, C. J. Am. Chem. Soc. 2006, 128 (50), 1597815979.

104) Shih, W. M.; Quispe, J. D.; Joyce, G. F. J. Biomol. Struct. Dyn. 2005, 22 (6), 762.

105) Rothemund, P. W. K. Nature 2006, 440 (7082), 297-302.

106) Douglas, S. M.; Dietz, H.; Liedl, T.; Hogberg, B.; Graf, F.; Shih, W. M. Nature 2009, 459 (7250), 1154-1154.

107) Julin, S.; Nummelin, S.; Kostiainen, M. A.; Linko, V. J. Nanopart. Res. 2018, 20 (5), 119.

108) Mazid, R. R.; Si, K. J.; Cheng, W. L. Methods 2014, 67 (2), 215-226. 
109) Jones, M. R.; Osberg, K. D.; Macfarlane, R. J.; Langille, M. R.; Mirkin, C. A. Chem. Rev. 2011, 111 (6), 3736-3827.

110) Alivisatos, A. P.; Johnsson, K. P.; Peng, X.; Wilson, T. E.; Loweth, C. J.; Bruchez, M. P.; Schultz, P. G. Nature 1996, 382 (6592), 609-611.

111) Hurst, S. J.; Lytton-Jean, A. K. R.; Mirkin, C. A. Anal. Chem. 2006, 78 (24), 83138318.

112) Pranata, J.; Wierschke, S. G.; Jorgensen, W. L. J. Am. Chem. Soc. 1991, 113 (8), 2810-2819.

113) Mucic, R. C.; Storhoff, J. J.; Mirkin, C. A.; Letsinger, R. L. J. Am. Chem. Soc. 1998, 120 (48), 12674-12675.

114) Park, S. Y.; Lytton-Jean, A. K. R.; Lee, B.; Weigand, S.; Schatz, G. C.; Mirkin, C. A. Nature 2008, 451 (7178), 553-556.

115) Zhang, Y.; Lu, F.; Yager, K. G.; van der Lelie, D.; Gang, O. Nat. Nanotechnol. 2013, 8 (11), 865-872.

116) Macfarlane, R. J.; Lee, B.; Hill, H. D.; Senesi, A. J.; Seifert, S.; Mirkin, C. A. Proc. Natl. Acad. Sci. U.S.A. 2009, 106 (26), 10493-8.

117) Thaner, R. V.; Kim, Y.; Li, T. I. N. G.; Macfarlane, R. J.; Nguyen, S. T.; de la Cruz, M. O.; Mirkin, C. A. Nano Lett. 2015, 15 (8), 5545-5551.

118) Macfarlane, R. J.; Jones, M. R.; Senesi, A. J.; Young, K. L.; Lee, B.; Wu, J.; Mirkin, C. A. Angew. Chem. Int. Ed. 2010, 49 (27), 4589-4592.

119) Macfarlane, R. J.; Thaner, R. V.; Brown, K. A.; Zhang, J.; Lee, B.; Nguyen, S. T.; Mirkin, C. A. Proc. Natl. Acad. Sci. U.S.A. 2014, 111 (42), 14995-15000.

120) Sun, D.; Gang, O. J. Am. Chem. Soc. 2011, 133 (14), 5252-5254.

121) Sun, D.; Gang, O. Langmuir 2013, 29 (23), 7038-7046.

122) Millstone, J. E.; Georganopoulou, D. G.; Xu, X.; Wei, W.; Li, S.; Mirkin, C. A. Small 2008, 4 (12), 2176-80.

123) Xu, L.; Kuang, H.; Xu, C.; Ma, W.; Wang, L.; Kotov, N. A. J. Am. Chem. Soc. 2012, 134 (3), 1699-709.

124) Fang, A.; White, S.; Jain, P. K.; Zamborini, F. P. Nano Lett. 2015, 15 (1), 542-548.

125) Caswell, K. K.; Wilson, J. N.; Bunz, U. H. F.; Murphy, C. J. J. Am. Chem. Soc. 2003, 125 (46), 13914-13915. 
126) Rossi, L.; Sacanna, S.; Irvine, W. T. M.; Chaikin, P. M.; Pine, D. J.; Philipse, A. P. Soft Matter 2011, 7 (9), 4139-4142.

127) Zhu, D.; Li, W.; Ma, H. R. J. Phys. Condens. Matter 2003, 15 (49), 8281-8290.

128) O'Brien, M. N.; Jones, M. R.; Lee, B.; Mirkin, C. A. Nat. Mater. 2015, 14 (8), 8339.

129) Lu, F.; Yager, K. G.; Zhang, Y.; Xin, H.; Gang, O. Nat. Commun. 2015, 6, 6912.

130) Lee, J. H.; Mahmoud, M. A.; Sitterle, V.; Sitterle, J.; Meredith, J. C. J. Am. Chem. Soc. 2009, 131 (14), 5048-9.

131) Lee, J.-H.; Mahmoud, M. A.; Sitterle, V. B.; Sitterle, J. J.; Meredith, J. C. Chem. Mater. 2009, 21 (23), 5654-5663.

132) Jeong, C. J.; In, I.; Park, S. Y. Surf. Interface Anal. 2015, 47 (2), 253-258.

133) Ishida, T.; Kuroda, K.; Kinoshita, N.; Minagawa, W.; Haruta, M. J. Colloid Interface Sci. 2008, 323 (1), 105-111.

134) Peceros, K. E.; Xu, X. D.; Bulcock, S. R.; Cortie, M. B. J. Mater. Chem. B 2005, 109 (46), 21516-21520.

135) Zhang, J. H.; Liu, J. B.; Wang, S. Z.; Zhan, P.; Wang, Z. L.; Ming, N. B. Adv. Funct. Mater. 2004, 14 (11), 1089-1096.

136) Lee, J.-H.; Oh, J. S.; Lee, P. C.; Kim, D. O.; Lee, Y.; Nam, J.-D. J. Electron. Mater. 2008, 37 (10), 1648-1652.

137) Cassagneau, T.; Caruso, F. Adv. Mater. 2002, 14 (10), 732-+.

138) Kato, N.; Caruso, F. J. Phys. Chem. B 2005, 109 (42), 19604-19612.

139) Zhang, J. H.; Bai, L. T.; Zhang, K.; Cui, Z. C.; Zhang, G.; Yang, B. J. Mater. Chem. 2003, 13 (3), 514-517.

140) Ji, T. H.; Lirtsman, V. G.; Avny, Y.; Davidov, D. Adv. Mater. 2001, 13 (16), 1253 1256.

141) Wu, L.-A.; Li, W.-E.; Lin, D.-Z.; Chen, Y.-F. Sci. Rep. 2017, 7 (1), 13066.

142) Zhang, N. C.; Yu, X.; Hu, J. Q.; Xue, F.; Ding, E. Y. Rsc Adv. 2013, 3 (33), 1374013747.

143) Hu, J. W.; Li, J. F.; Ren, B.; Wu, D. Y.; Sun, S. G.; Tian, Z. Q. J. Phys. Chem. C 2007, 111 (3), 1105-1112. 
144) Xu, X.; Rosi, N. L.; Wang, Y.; Huo, F.; Mirkin, C. A. J. Am. Chem. Soc. 2006, 128 (29), 9286-9287.

145) Maye, M. M.; Nykypanchuk, D.; Cuisinier, M.; van der Lelie, D.; Gang, O. Nat. Mater. 2009, 8 (5), 388-391.

146) Xu, X.; Rosi, N. L.; Wang, Y.; Huo, F.; Mirkin, C. A. J. Am. Chem. Soc. 2006, 128 (29), 9286-9287.

147) Yin, T.; Liu, X.; Wang, J. Z.; An, Y. L.; Zhang, Z. K.; Shi, L. Q. Rsc Adv. 2015, 5 (59), 47458-47465.

148) Wen, F.; Zhang, W. Q.; Wei, G. W.; Wang, Y.; Zhang, J. Z.; Zhang, M. C.; Shi, L. Q. Chem. Mater. 2008, 20 (6), 2144-2150.

149) Hirsch, L. R.; Jackson, J. B.; Lee, A.; Halas, N. J.; West, J. Anal. Chem. 2003, 75 (10), 2377-2381.

150) Siiman, O.; Burshteyn, A. J. Phys. Chem. B 2000, 104 (42), 9795-9810.

151) Rogach, A.; Susha, A.; Caruso, F.; Sukhorukov, G.; Kornowski, A.; Kershaw, S.; Möhwald, H.; Eychmüller, A.; Weller, H. Adv. Mater. 2000, 12 (5), 333-337.

152) Zhou, C.; Tsai, T. H.; Adler, D. C.; Lee, H. C.; Cohen, D. W.; Mondelblatt, A.; Wang, Y. H.; Connolly, J. L.; Fujimoto, J. G. Opt. Lett. 2010, 35 (5), 700-702.

153) Hirsch, L. R.; Stafford, R. J.; Bankson, J. A.; Sershen, S. R.; Rivera, B.; Price, R. E.; Hazle, J. D.; Halas, N. J.; West, J. L. Proc. Natl. Acad. Sci. U.S.A. 2003, 100 (23), 13549-13554.

154) Lan, X.; Chen, Z.; Liu, B.-J.; Ren, B.; Henzie, J.; Wang, Q. Small 2013, 9 (13), 2308-2315.

155) Zhu, C.; Wang, M.; Dong, J.; Zhou, C.; Wang, Q. Langmuir 2018, 34 (49), 14963 14968.

156) Pal, S.; Deng, Z.; Wang, H.; Zou, S.; Liu, Y.; Yan, H. J. Am. Chem. Soc. 2011, 133 (44), 17606-17609.

157) Coronado, E. A.; Encina, E. R.; Stefani, F. D. Nanoscale 2011, 3 (10), 4042-4059.

158) Acuna, G. P.; Möller, F. M.; Holzmeister, P.; Beater, S.; Lalkens, B.; Tinnefeld, P. Science 2012, 338 (6106), 506-510.

159) Puchkova, A.; Vietz, C.; Pibiri, E.; Wünsch, B.; Sanz Paz, M.; Acuna, G. P.; Tinnefeld, P. Nano Lett. 2015, 15 (12), 8354-8359. 
160) Zheng, C. Y.; Palacios, E.; Zhou, W.; Hadibrata, W.; Sun, L.; Huang, Z.; Schatz, G. C.; Aydin, K.; Mirkin, C. A. Adv. Mater. 2019, 31 (41), 1904448.

161) Zheng, Y.; Thai, T.; Reineck, P.; Qiu, L.; Guo, Y.; Bach, U. Adv. Funct. Mater. 2013, 23 (12), 1519-1526.

162) Zhan, P.; Wen, T.; Wang, Z.-g.; He, Y.; Shi, J.; Wang, T.; Liu, X.; Lu, G.; Ding, B. Angew. Chem. Int. Ed. Engl. 2018, 57 (11), 2846-2850.

163) Zhou, C.; Yang, Y.; Li, H.; Gao, F.; Song, C.; Yang, D.; Xu, F.; Liu, N.; Ke, Y.; Su, S.; Wang, P. Nano Lett. 2020, 20 (5), 3155-3159.

164) Patel, D.; James, K. T.; O’Toole, M.; Zhang, G.; Keynton, R. S.; Gobin, A. M. J. Colloid Interface Sci. 2015, 441, 10-16.

165) Chowdhury, E.; Grapperhaus, C. A.; O’Toole, M. G. J. Nanopart. Res. 2020, 22 (6), 142.

166) Yilbas, B. S.; Hassan, G.; Al-Sharafi, A.; Ali, H.; Al-Aqeeli, N.; Al-Sarkhi, A. Sci. Rep. 2018, 8 (1), 2984.

167) Sharma, J. N.; Pattadar, D. K.; Mainali, B. P.; Zamborini, F. P. Anal. Chem. 2018, 90 (15), 9308-9314.

168) Soto, C. M.; Srinivasan, A.; Ratna, B. R. J. Am. Chem. Soc. 2002, 124 (29), 85088509.

169) Geerts, N.; Eiser, E. Soft Matter 2010, 6 (19), 4647-4660.

170) O’Brien, M. N.; Lin, H.-X.; Girard, M.; Olvera de la Cruz, M.; Mirkin, C. A. J. Am. Chem. Soc. 2016, 138 (44), 14562-14565.

171) Nie, Z.; Fava, D.; Rubinstein, M.; Kumacheva, E. J. Am. Chem. Soc. 2008, 130 (11), 3683-3689.

172) Zhang, Q.; Deng, Y.-X.; Luo, H.-X.; Shi, C.-Y.; Geise, G. M.; Feringa, B. L.; Tian, H.; Qu, D.-H. J. Am. Chem. Soc. 2019, 141 (32), 12804-12814.

173) Kang, C.; Honciuc, A. J. Phys. Chem. Lett. 2018, 9 (6), 1415-1421.

174) Pang, X.; Wan, C.; Wang, M.; Lin, Z. Angew. Chem. Int. Ed. 2014, 53 (22), 55245538.

175) Poggi, E.; Gohy, J.-F. Colloid. Polym. Sci. 2017, 295 (11), 2083-2108.

176) Crossley, S.; Faria, J.; Shen, M.; Resasco, D. E. Science 2010, 327 (5961), 68-72. 
177) Li, X.; Zhou, L.; Wei, Y.; El-Toni, A. M.; Zhang, F.; Zhao, D. J. Am. Chem. Soc. 2014, 136 (42), 15086-15092.

178) Yu, Z.; Wang, C.-F.; Ling, L.; Chen, L.; Chen, S. Angew. Chem. Int. Ed. 2012, 51 (10), 2375-2378.

179) Zhao, Y. J.; Gu, H. C.; Xie, Z. Y.; Shum, H. C.; Wang, B. P.; Gu, Z. Z. J. Am. Chem. Soc. 2013, 135 (1), 54-57.

180) Jurado-Sanchez, B.; Sattayasamitsathit, S.; Gao, W.; Santos, L.; Fedorak, Y.; Singh, V. V.; Orozco, J.; Galarnyk, M.; Wang, J. Small 2015, 11 (4), 499-506.

181) Tanaka, T.; Okayama, M.; Minami, H.; Okubo, M. Langmuir 2010, 26 (14), 1173211736.

182) Parmar, J.; Ma, X.; Katuri, J.; Simmchen, J.; Stanton, M. M.; Trichet-Paredes, C.; Soler, L.; Sanchez, S. Sci. Technol. Adv. Mater. 2015, 16 (1), 9.

183) Wang, X.; Feng, X.; Ma, G.; Yao, L.; Ge, M. Adv Mater 2016, 28 (16), 3131-7.

184) Zahn, N.; Kickelbick, G. Colloids Surf. A Physicochem. Eng. Asp. 2014, 461, 142150.

185) Sheng, L.; Chen, H.; Fu, W.; Li, Z. Langmuir 2015, 31 (44), 11964-11970.

186) Hong, L.; Cacciuto, A.; Luijten, E.; Granick, S. Nano Lett. 2006, 6 (11), 25102514.

187) Roh, K. H.; Martin, D. C.; Lahann, J. Nat. Mater. 2005, 4 (10), 759-763.

188) Lone, S.; Cheong, I. W. RSC Adv. 2014, 4 (26), 13322-13333.

189) Walther, A.; André, X.; Drechsler, M.; Abetz, V.; Müller, A. H. E. J. Am. Chem. Soc. 2007, 129 (19), 6187-6198.

190) Liu, K.; Nie, Z. H.; Zhao, N. N.; Li, W.; Rubinstein, M.; Kumacheva, E. Science 2010, 329 (5988), 197-200.

191) Bhaskar, S.; Pollock, K. M.; Yoshida, M.; Lahann, J. Small 2010, 6 (3), 404-411.

192) Uzun, O.; Hu, Y.; Verma, A.; Chen, S.; Centrone, A.; Stellacci, F. Chem. Commun. 2008, (2), 196-198.

193) Zhang, X. D.; Wu, D.; Shen, X.; Liu, P. X.; Yang, N.; Zhao, B.; Zhang, H.; Sun, Y. M.; Zhang, L. A.; Fan, F. Y. Int. J. Nanomedicine 2011, 6, 2071-81.

194) Norgaard, K.; Weygand, M. J.; Kjaer, K.; Brust, M.; Bjornholm, T. Faraday Discuss. 2004, 125, 221-233. 
195) Lee, H. Y.; Shin, S. H. R.; Abezgauz, L. L.; Lewis, S. A.; Chirsan, A. M.; Danino, D. D.; Bishop, K. J. M. J. Am. Chem. Soc. 2013, 135 (16), 5950-5953.

196) Mezni, A.; Dammak, T.; Fkiri, A.; Mlayah, A.; Abid, Y.; Smiri, L. S. J. Phys. Chem. C 2014, 118 (31), 17956-17967.

197) Xu, X. Y.; Rosi, N. L.; Wang, Y. H.; Huo, F. W.; Mirkin, C. A. J. Am. Chem. Soc. 2006, 128 (29), 9286-9287.

198) Si, K. J.; Chen, Y.; Shi, Q.; Cheng, W. Adv. Sci. 2018, 5 (1), 1700179.

199) Dutta, A.; Chakraborty, J.; Prasad, B. L. V.; Sahu, P. J. Colloid Interface Sci. 2014, 420, 41-49.

200) Ross, M. B.; Ku, J. C.; Vaccarezza, V. M.; Schatz, G. C.; Mirkin, C. A. Nat. Nanotechnol. 2015, 10 (5), 453-458.

201) Alaeian, H.; Dionne, J. A. Opt. Express 2012, 20 (14), 15781-96.

202) Wang, D.; Yang, A.; Hryn, A. J.; Schatz, G. C.; Odom, T. W. ACS Photonics 2015, 2 (12), 1789-1794.

203) Chen, J.; Guo, L.; Qiu, B.; Lin, Z.; Wang, T. Mater. Chem. Front. 2018, 2 (5), 835860 .

204) Wang, W.; Ramezani, M.; Väkeväinen, A. I.; Törmä, P.; Rivas, J. G.; Odom, T. W. Mater. Today 2018, 21 (3), 303-314.

205) Nie, Z.; Petukhova, A.; Kumacheva, E. Nat. Nanotechnol. 2010, 5 (1), 15-25.

206) Schulz, F.; Pavelka, O.; Lehmkühler, F.; Westermeier, F.; Okamura, Y.; Mueller, N. S.; Reich, S.; Lange, H. Nat. Commun. 2020, 11 (1), 3821.

207) Tao, A.; Sinsermsuksakul, P.; Yang, P. Nat. Nanotechnol. 2007, 2 (7), 435-40.

208) Mendes, P. M.; Jacke, S.; Critchley, K.; Plaza, J.; Chen, Y.; Nikitin, K.; Palmer, R. E.; Preece, J. A.; Evans, S. D.; Fitzmaurice, D. Langmuir 2004, 20 (9), 3766-3768.

209) Werts, M. H. V.; Lambert, M.; Bourgoin, J.-P.; Brust, M. Nano Lett. 2002, 2 (1), 43-47.

210) Tao, A. R.; Huang, J.; Yang, P. Acc. Chem. Res. 2008, 41 (12), 1662-73.

211) Li, P.; Li, Y.; Zhou, Z.-K.; Tang, S.; Yu, X.-F.; Xiao, S.; Wu, Z.; Xiao, Q.; Zhao, Y.; Wang, H.; Chu, P. K. Adv. Mater. 2016, 28 (13), 2511-2517.

212) Wei, W.; Wang, Y.; Ji, J.; Zuo, S.; Li, W.; Bai, F.; Fan, H. Nano Lett. 2018, 18 (7), 4467-4472. 
213) Pang, J.; Xiong, S.; Jaeckel, F.; Sun, Z.; Dunphy, D.; Brinker, C. J. J. Am. Chem. Soc. 2008, 130 (11), 3284-3285.

214) Shi, Q.; Di, W.; Dong, D.; Yap, L. W.; Li, L.; Zang, D.; Cheng, W. ACS Nano 2019, 13 (5), 5243-5250.

215) Radha, B.; Senesi, A. J.; O’Brien, M. N.; Wang, M. X.; Auyeung, E.; Lee, B.; Mirkin, C. A. Nano Lett. 2014, 14 (4), 2162-2167.

216) Mazid, R. R.; Si, K. J.; Cheng, W. Methods 2014, 67 (2), 215-226.

217) Kumar, A.; Hwang, J.-H.; Kumar, S.; Nam, J.-M. Chem. Commun. 2013, 49 (26), 2597-2609.

218) Tan, S. J.; Campolongo, M. J.; Luo, D.; Cheng, W. Nat. Nanotechnol. 2011, 6 (5), 268-76.

219) Young, K. L.; Ross, M. B.; Blaber, M. G.; Rycenga, M.; Jones, M. R.; Zhang, C.; Senesi, A. J.; Lee, B.; Schatz, G. C.; Mirkin, C. A. Adv. Mater. 2014, 26 (4), 653-659.

220) Hatab, N. A.; Hsueh, C.-H.; Gaddis, A. L.; Retterer, S. T.; Li, J.-H.; Eres, G.; Zhang, Z.; Gu, B. Nano Lett. 2010, 10 (12), 4952-4955.

221) Kausar, A. S. M. Z.; Reza, A. W.; Latef, T. A.; Ullah, M. H.; Karim, M. E. Sensors (Basel). 2015, 15 (4), 8787-8831.

222) Mayer, K. M.; Hafner, J. H. Chem. Rev. 2011, 111 (6), 3828-3857.

223) Masson, J. F. ACS Sens. 2017, 2 (1), 16-30.

224) Gramotnev, D. K.; Bozhevolnyi, S. I. Nat. Photonics 2010, 4 (2), 83-91.

225) Demirel, G. J. Mater. Chem. C. 2018, 6.

226) Pilo-Pais, M.; Watson, A.; Demers, S.; LaBean, T. H.; Finkelstein, G. Nano Lett. 2014, 14 (4), 2099-2104.

227) Reguera, J.; Langer, J.; Jimenez de Aberasturi, D.; Liz-Marzan, L. M. Chem. Soc. Rev. 2017, 46 (13), 3866-3885.

228) Li, J.-F.; Li, C.-Y.; Aroca, R. F. Chem. Soc. Rev. 2017, 46 (13), 3962-3979.

229) Ray, P. C. Chem. Rev. 2010, 110 (9), 5332-5365.

230) Yilmaz, M.; Senlik, E.; Biskin, E.; Yavuz, M. S.; Tamer, U.; Demirel, G. Phys. Chem. Chem. Phys. 2014, 16 (12), 5563-70.

231) Park, J.-E.; Kim, J.; Nam, J.-M. Chem. Sci. 2017, 8 (7), 4696-4704. 
232) Chen, X.; Jiang, C.; Yu, S. CrystEngComm 2014, 16 (43), 9959-9973.

233) Scarabelli, L.; Coronado-Puchau, M.; Giner-Casares, J. J.; Langer, J.; Liz-Marzán, L. M. ACS Nano 2014, 8 (6), 5833-5842.

234) Lim, D.-K.; Jeon, K.-S.; Kim, H. M.; Nam, J.-M.; Suh, Y. D. Nat. Mater. 2010, 9 (1), 60-67.

235) Wei, H.; Xu, H. Nanoscale 2013, 5 (22), 10794-10805.

236) Lakowicz, J. R.; Geddes, C. D.; Gryczynski, I.; Malicka, J.; Gryczynski, Z.; Aslan, K.; Lukomska, J.; Matveeva, E.; Zhang, J.; Badugu, R.; Huang, J. J. Fluoresc. 2004, 14 (4), 425-441.

237) Chen, H.-Y.; Lin, M.-H.; Wang, C.-Y.; Chang, Y.-M.; Gwo, S. J. Am. Chem. Soc. 2015, 137 (42), 13698-13705.

238) Kasani, S.; Curtin, K.; Wu, N. Nanophotonics 2019, 8 (12), 2065.

239) Lee, H. K.; Lee, Y. H.; Koh, C. S. L.; Phan-Quang, G. C.; Han, X.; Lay, C. L.; Sim, H. Y. F.; Kao, Y.-C.; An, Q.; Ling, X. Y. Chem. Soc. Rev. 2019, 48 (3), 731-756.

240) Emtias Chowdhury, C. A. G., Martin G O'Toole. J. Mater. Chem. C (In peer review) 2020.

241) Auer, S.; Frenkel, D. Nature 2001, 413 (6857), 711-713.

242) Phillips, C. L.; Glotzer, S. C. J. Chem. Phys. 2012, 137 (10), 104901.

243) Laramy, C. R.; O’Brien, M. N.; Mirkin, C. A. Nat. Rev. Mater. 2019, 4 (3), 201224.

244) Lan, S.; Guo, C.; Zhou, W.; Ren, Y.; Almer, J.; Pei, C.; Hahn, H.; Liu, C.-T.; Feng, T.; Wang, X.-L.; Gleiter, H. Commun. Phys. 2019, 2 (1), 117.

245) Nykypanchuk, D.; Maye, M. M.; van der Lelie, D.; Gang, O. Nature 2008, 451 (7178), 549-552.

246) Sinha, S. S.; Jones, S.; Demeritte, T.; Chavva, S. R.; Shi, Y.; Burrell, J.; Pramanik, A.; Ray, P. C. J. Phys. Chem. C 2016, 120 (8), 4546-4555.

247) Luo, B.; Kim, A.; Smith, J. W.; Ou, Z.; Wu, Z.; Kim, J.; Chen, Q. Nat. Commun. 2019, $10(1), 1815$.

248) Ou, Z.; Wang, Z.; Luo, B.; Luijten, E.; Chen, Q. Nat. Mater. 2020, 19 (4), 450-455.

249) Agarwal, U.; Escobedo, F. A. Nat. Mater. 2011, 10 (3), 230-235. 
250) Demirel, G.; Gieseking, R. L. M.; Ozdemir, R.; Kahmann, S.; Loi, M. A.; Schatz, G. C.; Facchetti, A.; Usta, H. Nat. Commun. 2019, 10 (1), 5502.

251) Li, C.; Huang, Y.; Lai, K.; Rasco, B. A.; Fan, Y. Food Control 2016, 65, 99-105.

252) Xu, T.; Wang, X.; Huang, Y.; Lai, K.; Fan, Y. Food Control 2019, 106, 106720.

253) Roy, S. D.; Ghosh, M.; Chowdhury, J. J. Raman. Spectrosc. 2015, 46.

254) Srichan, C.; Ekpanyapong, M.; Horprathum, M.; Eiamchai, P.; Nuntawong, N.; Phokharatkul, D.; Danvirutai, P.; Bohez, E.; Wisitsoraat, A.; Tuantranont, A. Sci. Rep. 2016, 6 (1), 23733.

255) Yilmaz, M. Phys. Chem. Chem. Phys. 2014, 16.

256) Xiao, G.-N.; Man, S.-Q. Chem. Phys. Lett. 2007, 447 (4), 305-309.

257) Pompa, P. P.; Martiradonna, L.; Torre, A. D.; Sala, F. D.; Manna, L.; De Vittorio, M.; Calabi, F.; Cingolani, R.; Rinaldi, R. Nat. Nanotechnol. 2006, 1 (2), 126-130.

258) Trojanowska, A.; Pazos-Perez, N.; Panisello, C.; Gumi, T.; Guerrini, L.; AlvarezPuebla, R. A. J. Colloid Interface Sci. 2015, 460, 128-134.

259) Westcott, S. L.; Oldenburg, S. J.; Lee, T. R.; Halas, N. J. Langmuir 1998, 14 (19), 5396-5401.

260) Strozyk, M. S.; Jimenez de Aberasturi, D.; Liz-Marzan, L. M. Chem. Rec. 2018, 18 (7-8), 807-818.

261) Wei, Q.-H.; Su, K.-H.; Zhang, X.-X.; Zhang, X., Engineering 'hot spots' for surface-enhanced Raman scattering. SPIE: 2003; Vol. 5221.

262) Belhout, S. A.; Baptista, F. R.; Devereux, S. J.; Parker, A. W.; Ward, A. D.; Quinn, S. J. Nanoscale 2019, 11 (42), 19884-19894.

263) Belhout, S. A.; Kim, J. Y.; Hinds, D. T.; Owen, N. J.; Coulter, J. A.; Quinn, S. J. Chem. Commun. 2016, 52 (100), 14388-14391.

264) Serrano-Montes, A. B.; Langer, J.; Henriksen-Lacey, M.; Jimenez de Aberasturi, D.; Solís, D. M.; Taboada, J. M.; Obelleiro, F.; Sentosun, K.; Bals, S.; Bekdemir, A.; Stellacci, F.; Liz-Marzán, L. M. J. Phys. Chem. C 2016, 120 (37), 20860-20868.

265) Hasell, T.; Yang, J.; Wang, W.; Li, J.; Brown, P. D.; Poliakoff, M.; Lester, E.; Howdle, S. M. J. Mater. Chem. 2007, 17 (41), 4382-4386. 
266) Burrows, N. D.; Vartanian, A. M.; Abadeer, N. S.; Grzincic, E. M.; Jacob, L. M.; Lin, W.; Li, J.; Dennison, J. M.; Hinman, J. G.; Murphy, C. J. J. Phys. Chem. Lett. 2016, 7 (4), 632-641.

267) Tebbe, M.; Maennel, M.; Fery, A.; Pazos-Perez, N.; Alvarez-Puebla, R. A. J. Phys. Chem. C 2014, 118 (48), 28095-28100.

268) McLintock, A.; Cunha-Matos, C. A.; Zagnoni, M.; Millington, O. R.; Wark, A. W. ACS Nano 2014, 8 (8), 8600-8609.

269) Park, S.; Lee, J.; Ko, H. ACS Appl. Mater. Interfaces 2017, 9 (50), 44088-44095.

270) Yang, Y.; Zhong, X. L.; Zhang, Q.; Blackstad, L. G.; Fu, Z. W.; Li, Z. Y.; Qin, D. Small 2014, 10 (7), 1430-7.

271) Alsammarraie, F. K.; Lin, M. J. Agric. Food Chem. 2017, 65 (3), 666-674.

272) Zhang, Z.; Yu, Q.; Li, H.; Mustapha, A.; Lin, M. J. Food Sci. 2015, 80 (2), N450-8.

273) Caglayan, M. G.; Kasap, E.; Cetin, D.; Suludere, Z.; Tamer, U. Microchimica Acta 2017, 184 (4), 1059-1067.

274) Chowdhury, E.; Rahaman, M. S.; Sathitsuksanoh, N.; Grapperhaus, C. A.; O’Toole, M. G. Nanotechnology 2020, 32 (2), 025506.

275) Dulkeith, E.; Morteani, A. C.; Niedereichholz, T.; Klar, T. A.; Feldmann, J.; Levi, S. A.; van Veggel, F. C.; Reinhoudt, D. N.; Moller, M.; Gittins, D. I. Phys. Rev. Lett. 2002, 89 (20), 203002.

276) Kang, K. A.; Wang, J. T.; O'Toole, M. G.; Nantz, M.; Moore, J. D.; Laulhe, S.; Achilefu, S., Sensitivity Enhancement of NIR Fluorescence Contrast Agent Utilizing Gold Nanoparticles. In Oxygen Transport to Tissue Xxxiii, Wolf, M.; Bucher, H. U.; Rudin, M.; VanHuffel, S.; Wolf, U.; Bruley, D. F.; Harrison, D. K., Eds. Springer-Verlag Berlin: Berlin, 2012; Vol. 737, pp 285-291.

277) Dubertret, B.; Calame, M.; Libchaber, A. J. Nat. Biotechnol. 2001, 19 (4), 365-70.

278) Wang, J.; O'Toole, M.; Massey, A.; Biswas, S.; Nantz, M.; Achilefu, S.; Kang, K. A., Highly Specific, NIR Fluorescent Contrast Agent with Emission Controlled by Gold Nanoparticle. In Oxygen Transport to Tissue Xxxii, LaManna, J. C.; Puchowicz, M. A.; Xu, K.; Harrison, D. K.; Bruley, D. F., Eds. 2011; Vol. 701, pp 149-154.

279) Benincasa, M.; Pacor, S.; Gennaro, R.; Scocchi, M. Antimicrob. Agents Chemother. 2009, 53 (8), 3501-3504.

280) Maxwell, D. J.; Taylor, J. R.; Nie, S. J. Am. Chem. Soc. 2002, 124 (32), 9606-9612. 
281) Hong, B.; Kang, K. A. Biosens. Bioelectron. 2006, 21 (7), 1333-8.

282) Dulkeith, E.; Ringler, M.; Klar, T. A.; Feldmann, J.; Muñoz Javier, A.; Parak, W. J. Nano Lett. 2005, 5 (4), 585-589.

283) Soller, T.; Ringler, M.; Wunderlich, M.; Klar, T. A.; Feldmann, J.; Josel, H. P.; Markert, Y.; Nichtl, A.; Kürzinger, K. Nano Lett. 2007, 7 (7), 1941-1946.

284) Karthikeyan, B. J. Appl. Phys. 2010, 108 (8), 084311.

285) Jin, R.; Wu, G.; Li, Z.; Mirkin, C. A.; Schatz, G. C. J. Am. Chem. Soc. 2003, 125 (6), 1643-1654.

286) Yang, S.; Li, B.; Slipchenko, M. N.; Akkus, A.; Singer, N. G.; Yeni, Y. N.; Akkus, O. J. Raman. Spectrosc. 2013, 44 (8), 1089-1095.

287) Haran, G.; Chuntonov, L. Chem. Rev. 2018, 118 (11), 5539-5580.

288) Mastroianni, A. J.; Claridge, S. A.; Alivisatos, A. P. J. Am. Chem. Soc. 2009, 131 (24), 8455-8459.

289) Hill, R. T.; Mock, J. J.; Hucknall, A.; Wolter, S. D.; Jokerst, N. M.; Smith, D. R.; Chilkoti, A. ACS Nano 2012, 6 (10), 9237-9246.

290) Panoiu, N. C.; Sha, W. E. I.; Lei, D. Y.; Li, G. C. J. Opt. 2018, 20 (8), 083001.

291) Oulton, R. F. Mater. Today 2012, 15 (1), 26-34.

292) Ai, B.; Song, C.; Bradley, L.; Zhao, Y. J. Phys. Chem. C 2018, 122 (36), 2093520944.

293) Fang, W.; Jia, S.; Chao, J.; Wang, L.; Duan, X.; Liu, H.; Li, Q.; Zuo, X.; Wang, L.; Wang, L.; Liu, N.; Fan, C. Sci. Adv. 2019, 5 (9), eaau4506.

294) Cui, X.; Lai, Y.; Qin, F.; Shao, L.; Wang, J.; Lin, H.-Q. Nanoscale 2020, 12 (3), 1975-1984.

295) Su, W.; Ding, Y.; Luo, Y.; Liu, Y. Results Phys. 2020, 16, 102833.

296) Okamoto, K.; Tanaka, D.; Degawa, R.; Li, X.; Wang, P.; Ryuzaki, S.; Tamada, K. Sci. Rep. 2016, 6 (1), 36165.

297) Yan, J.; Ma, C.; Liu, P.; Wang, C.; Yang, G. Light Sci. Appl. 2017, 6 (1), e16197e16197.

298) Khanikaev, A. B.; Wu, C.; Shvets, G. Nanophotonics 2013, 2 (4), 247-264.

299) Norton, S. J.; Vo-Dinh, T. Appl. Opt. 2016, 55 (10), 2611-8. 
300) Fan, J. A.; Bao, K.; Wu, C.; Bao, J.; Bardhan, R.; Halas, N. J.; Manoharan, V. N.; Shvets, G.; Nordlander, P.; Capasso, F. Nano Lett. 2010, 10 (11), 4680-4685.

301) Mukherjee, S.; Sobhani, H.; Lassiter, J. B.; Bardhan, R.; Nordlander, P.; Halas, N. J. Nano Lett. 2010, 10 (7), 2694-2701.

302) Wu, D.; Jiang, S.; Liu, X. J. Chem. Phys. 2012, 136 (3), 034502.

303) Zhang, S.; Bao, K.; Halas, N. J.; Xu, H.; Nordlander, P. Nano Lett. 2011, 11 (4), 1657-1663.

304) Liu, N.; Hentschel, M.; Weiss, T.; Alivisatos, A. P.; Giessen, H. Science 2011, 332 (6036), 1407-1410.

305) Lin, Q.-Y.; Mason, J. A.; Li, Z.; Zhou, W.; O’Brien, M. N.; Brown, K. A.; Jones, M. R.; Butun, S.; Lee, B.; Dravid, V. P.; Aydin, K.; Mirkin, C. A. Science 2018, 359 (6376), 669-672.

306) Kar, A.; Thambi, V.; Paital, D.; Joshi, G.; Khatua, S. Langmuir 2020, 36 (33), 9894-9899.

307) Kumar, J.; Wei, X.; Barrow, S.; Funston, A. M.; Thomas, K. G.; Mulvaney, P. Phys. Chem. Chem. Phys. 2013, 15 (12), 4258-4264.

308) Liu, J.; Kan, C.; Li, Y.; Xu, H.; Ni, Y.; Shi, D. Appl. Phys. Lett. 2014, 104 (25), 253105.

309) Liu, W.; Tagawa, M.; Xin, H. L.; Wang, T.; Emamy, H.; Li, H.; Yager, K. G.; Starr, F. W.; Tkachenko, A. V.; Gang, O. Science 2016, 351 (6273), 582-586.

310) Wang, Y.; Wang, Y.; Zheng, X.; Ducrot, É.; Lee, M.-G.; Yi, G.-R.; Weck, M.; Pine, D. J. J. Am. Chem. Soc. 2015, 137 (33), 10760-10766.

311) Emtias Chowdhury, C. A. G., Martin G O’Toole. Manuscript in Prep. 2020.

312) Piantanida, L.; Naumenko, D.; Lazzarino, M. RSC Adv. 2014, 4 (29), 15281-15287.

313) Chen, G.; Wang, Y.; Tan, L. H.; Yang, M.; Tan, L. S.; Chen, Y.; Chen, H. J. Am. Chem. Soc. 2009, 131 (12), 4218-4219.

314) Liu, T.-R.; Zhou, Z.-K.; Jin, C.; Wang, X. Plasmonics 2013, 8 (2), 885-890.

315) Hentschel, M.; Dregely, D.; Vogelgesang, R.; Giessen, H.; Liu, N. ACS Nano 2011, 5 (3), 2042-2050.

316) Zheng, C.; Jia, T.; Zhao, H.; Xia, Y.; Zhang, S.; Feng, D.; Sun, Z. RSC Adv. 2018, 8 (7), 3381-3391. 
317) Fan, J. A.; Wu, C.; Bao, K.; Bao, J.; Bardhan, R.; Halas, N. J.; Manoharan, V. N.; Nordlander, P.; Shvets, G.; Capasso, F. Science 2010, 328 (5982), 1135-1138.

318) Mirin, N. A.; Bao, K.; Nordlander, P. J. Phys. Chem. A 2009, 113 (16), 4028-4034.

319) Zhang; Horsch, M. A.; Lamm, M. H.; Glotzer, S. C. Nano Lett. 2003, 3 (10), 13411346.

320) Lee, K.; Zhang, L.; Yi, Y.; Wang, X.; Yu, Y. ACS Nano 2018, 12 (4), 3646-3657. 
APPENDIX A

SYNTHESIS, CHARACTERIZATION, AND ASYMMETRIC SURFACE FUNCTIONALIZATION OF GOLD

NANOPRISMS 


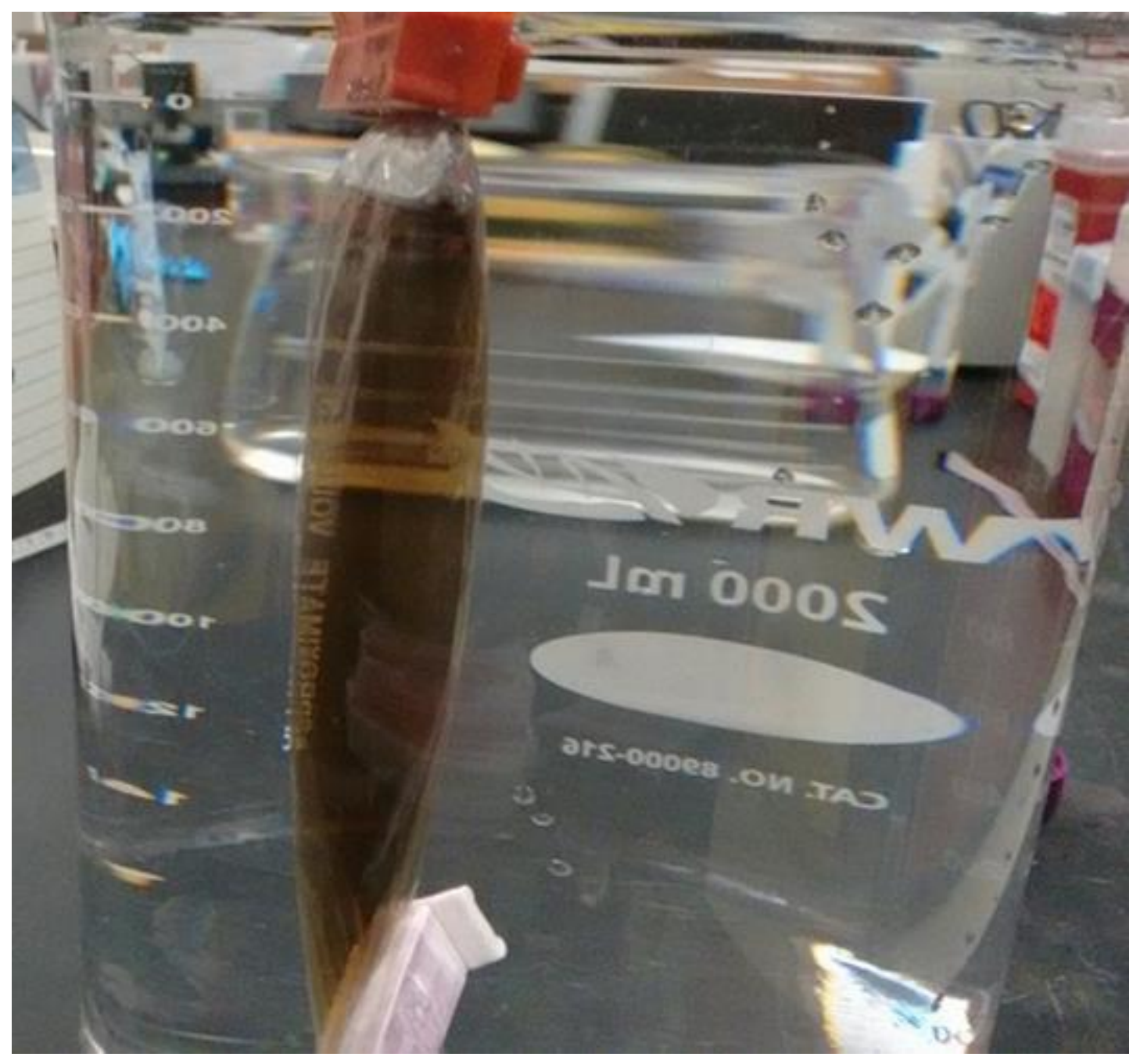

Figure A1: Picture of a typical reaction vessel made up of cellulose acetate with MWCO 12KD for the synthesis gold nanoprisms. 

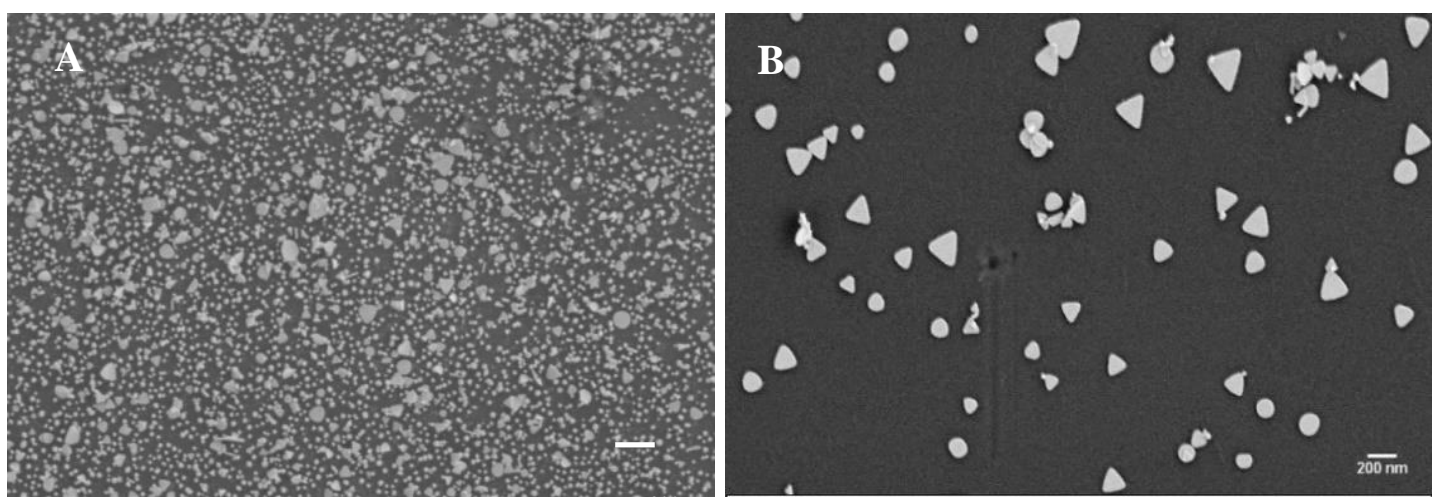

$\mathrm{C}$

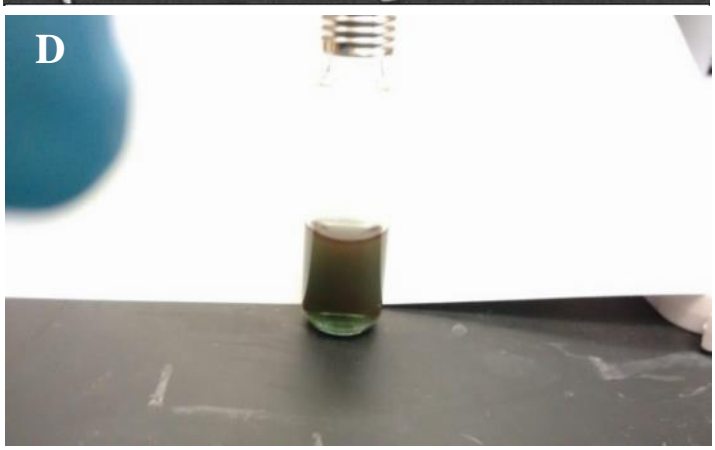

Figure A2: Representative SEM images of A) as synthesized gold nanoprism crude product, B) purified gold nanoprisms and an image of C) as synthesized gold nanoprism crude product (Brown), and D) purified gold nanoprisms (Green). 


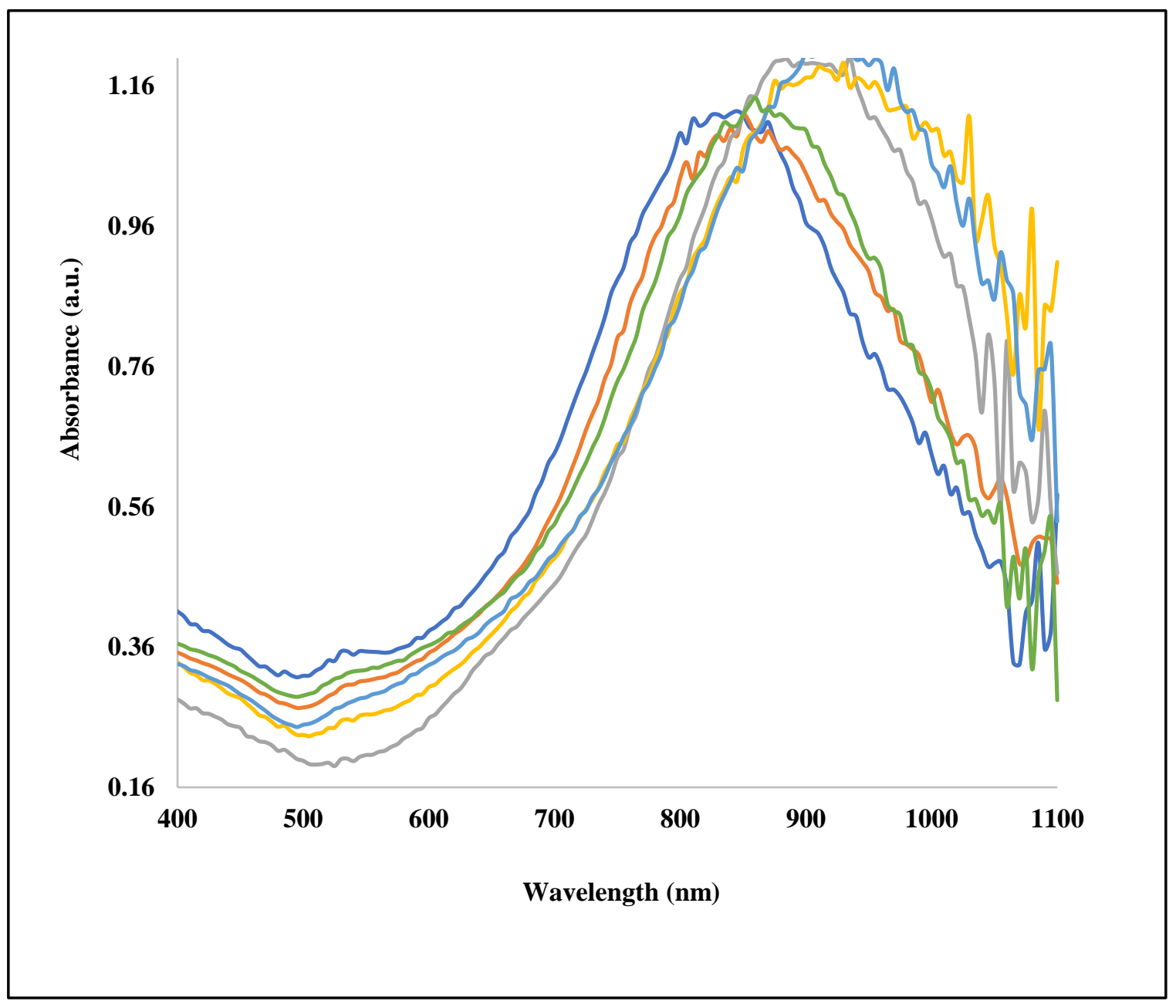

Figure A3: UV-Vis absorbance spectra of purified gold nanoprisms of different sizes. 


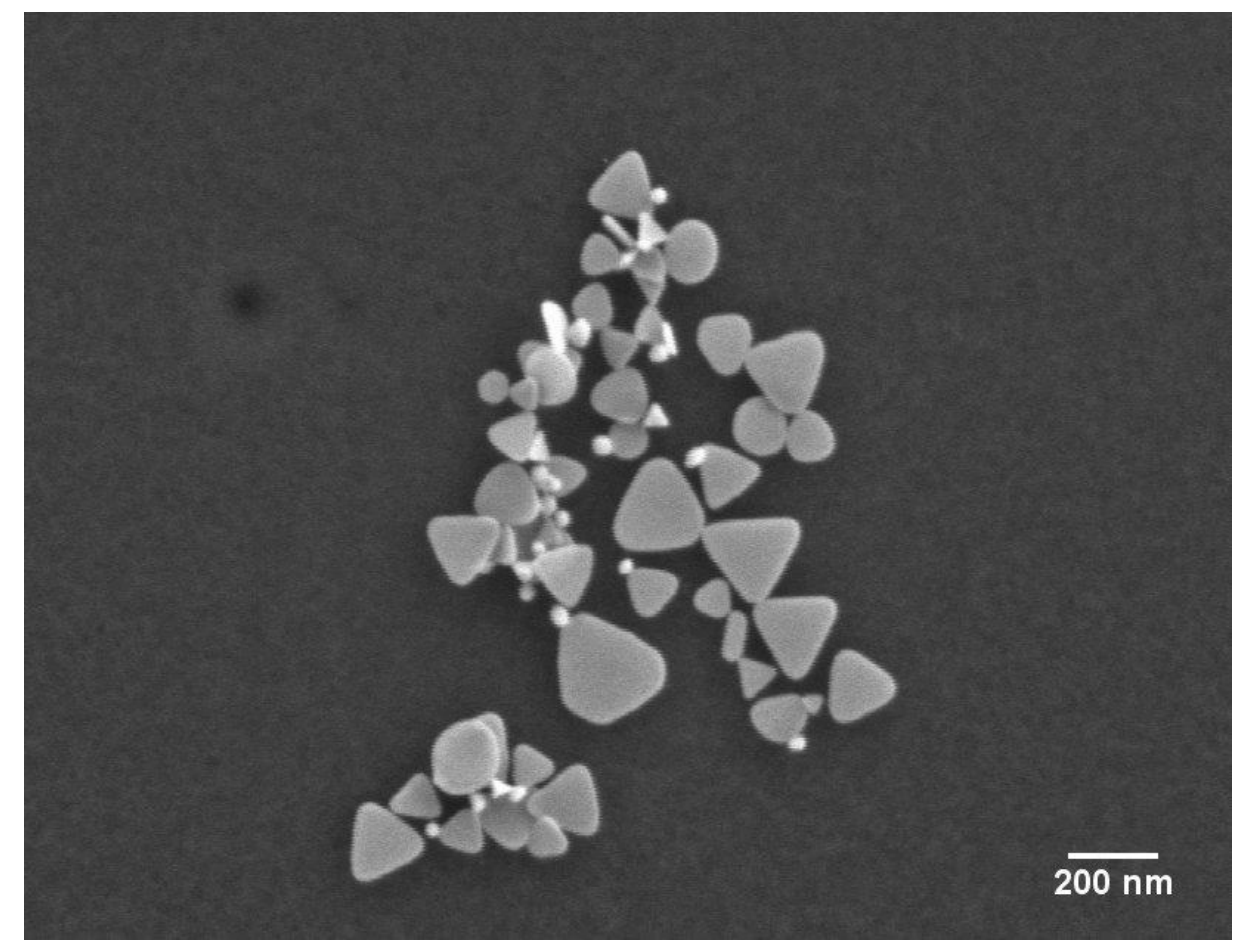

Figure A4: Representative SEM image of gold nanoprisms sample on a glass slide. 

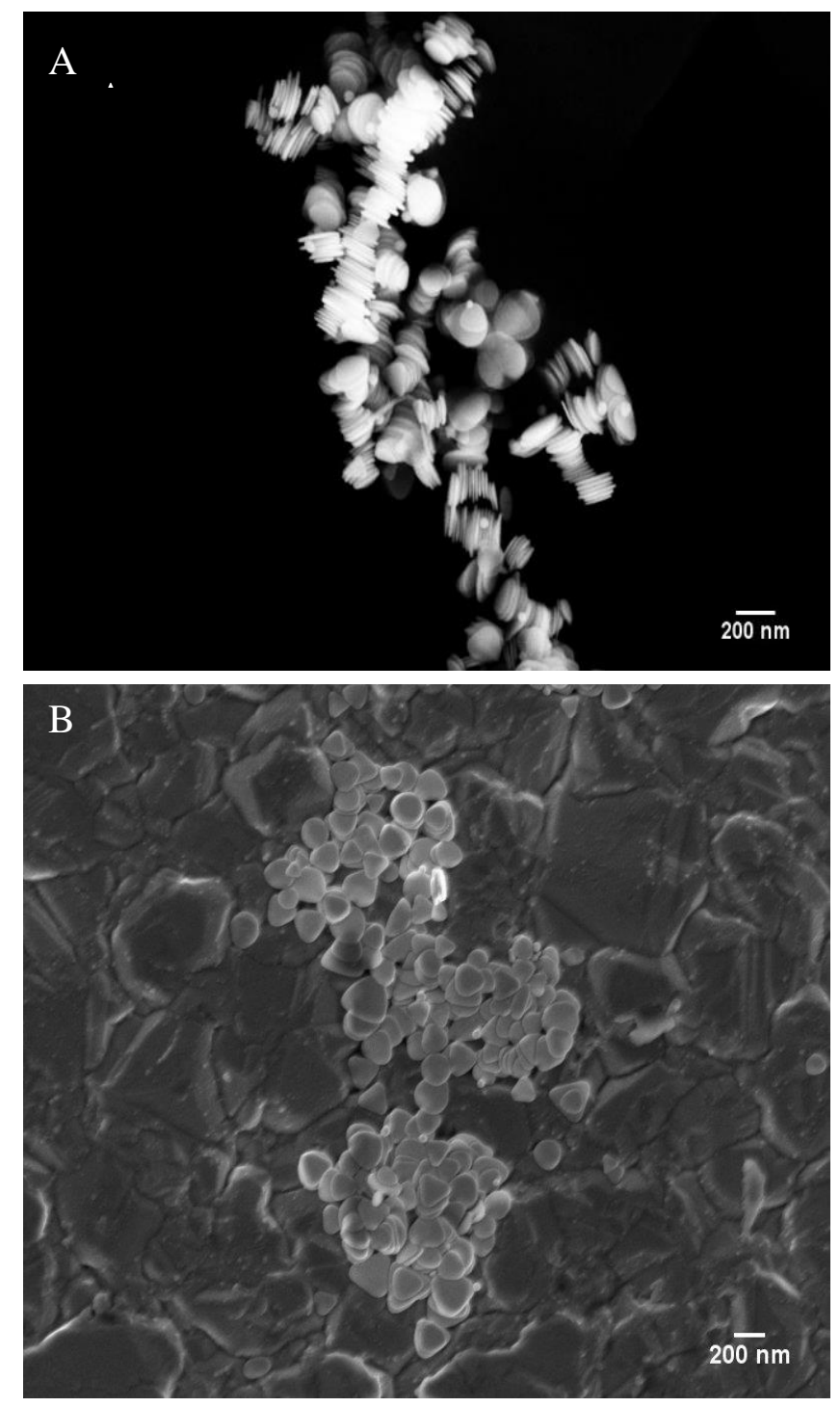

Figure A5: A) Representative SEM image of gold nanoprisms face-to-face arrangement after heating, which retained its structure after drop casting on the TEM grid, B) Representative SEM image showing the lack of face-toface assembly of gold nanoprisms without heating. 


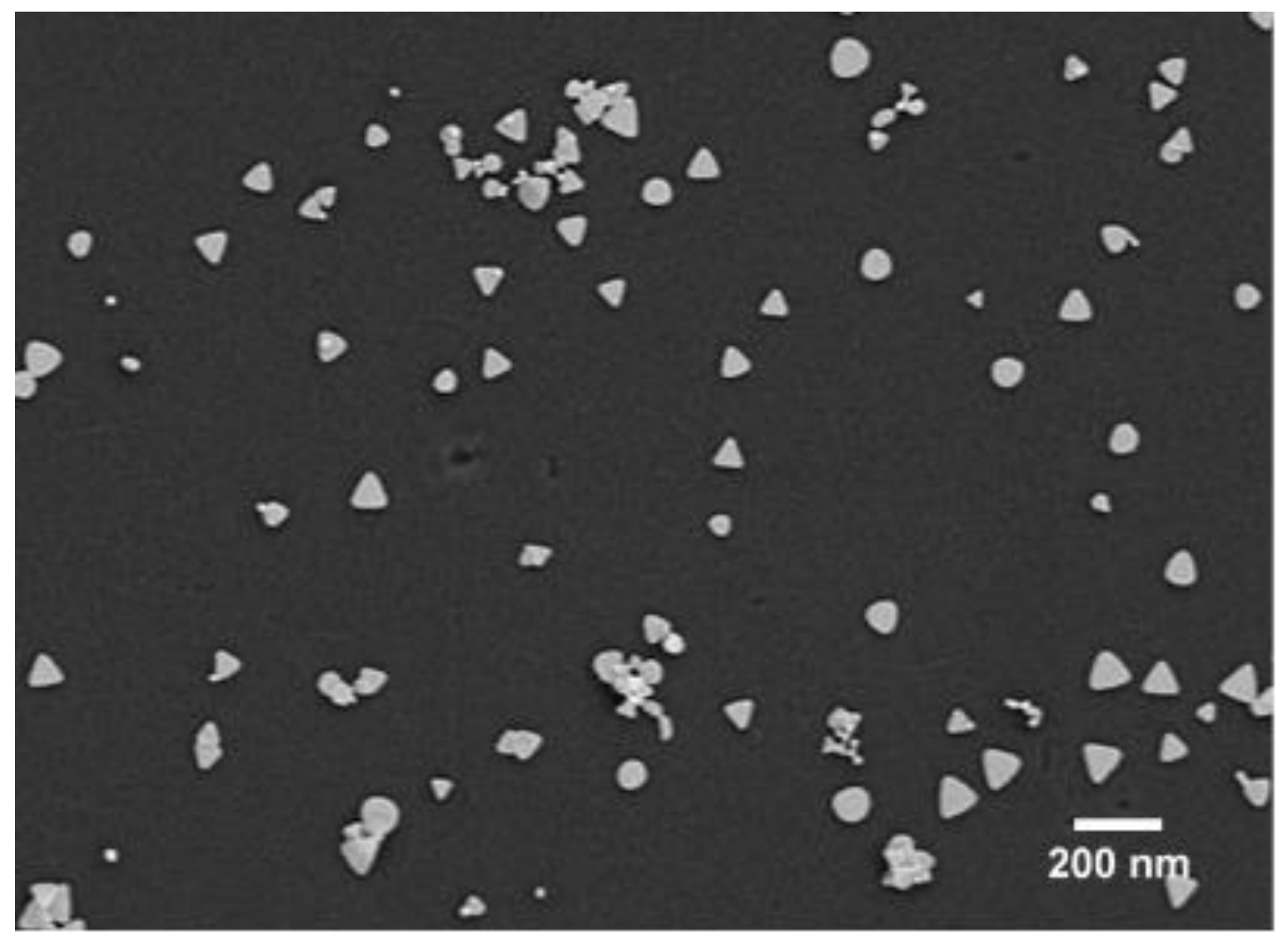

Figure A6: Representative SEM image of a monolayer of nanoprisms on the APTES functionalized glass surface. 


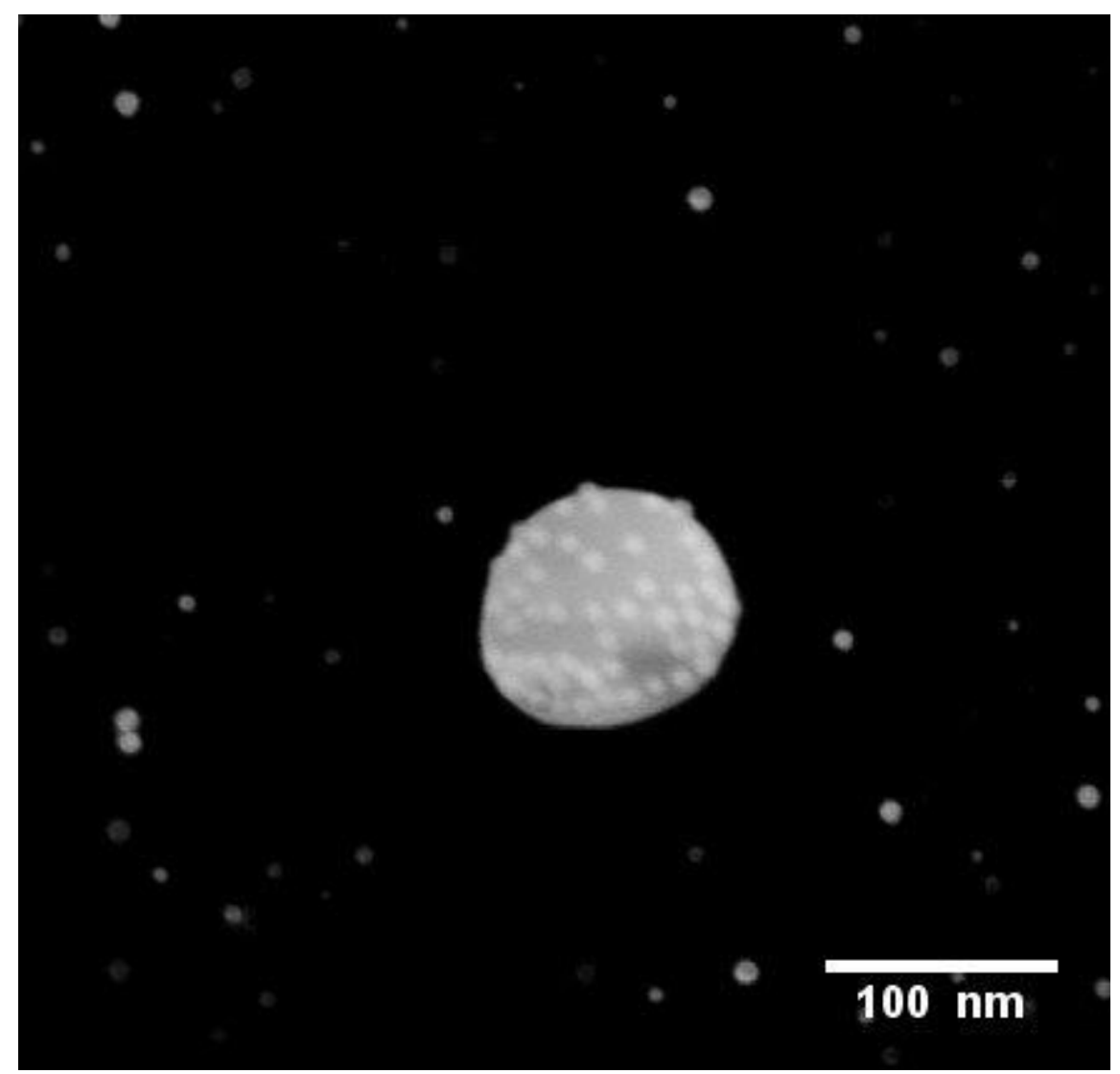

Figure A7: Representative STEM image of the conjugation of DNA-A" coated nanospheres with PEG | DNA-A Janus nanoprisms. 

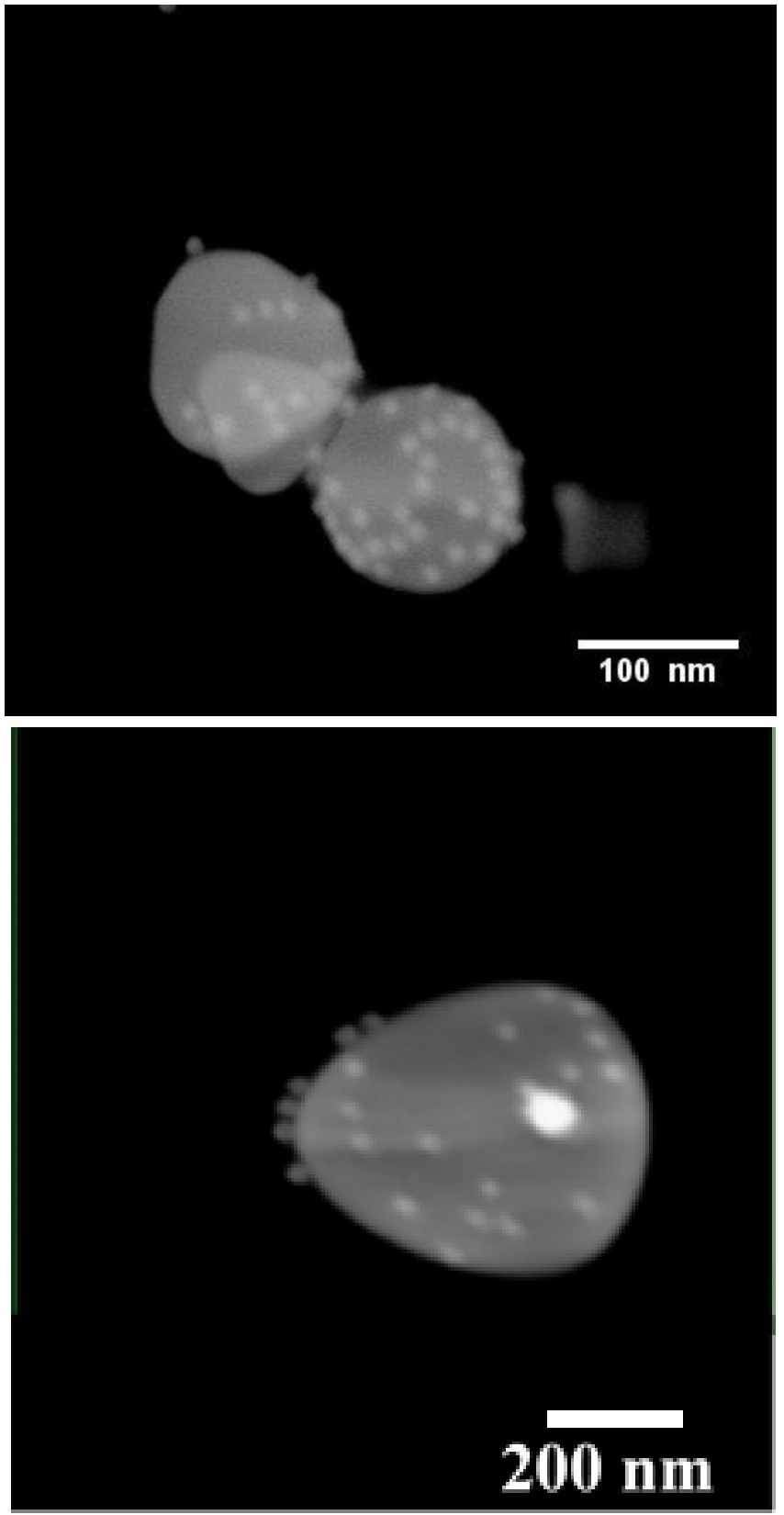

Figure A8: Additional STEM images of the conjugation of DNAA" coated nanospheres with PEG |DNA-A Janus nanoprisms. 


\section{APPENDIX B}

\section{ADDITIONAL SEM AND DARKFIELD MICROSCOPY CHARACTERIZATION OF NANOPRISM DIMERS, TRIMERS, SUPERLATTICES AND PS BEADS/ NANOPRISMS AGGREGATES}



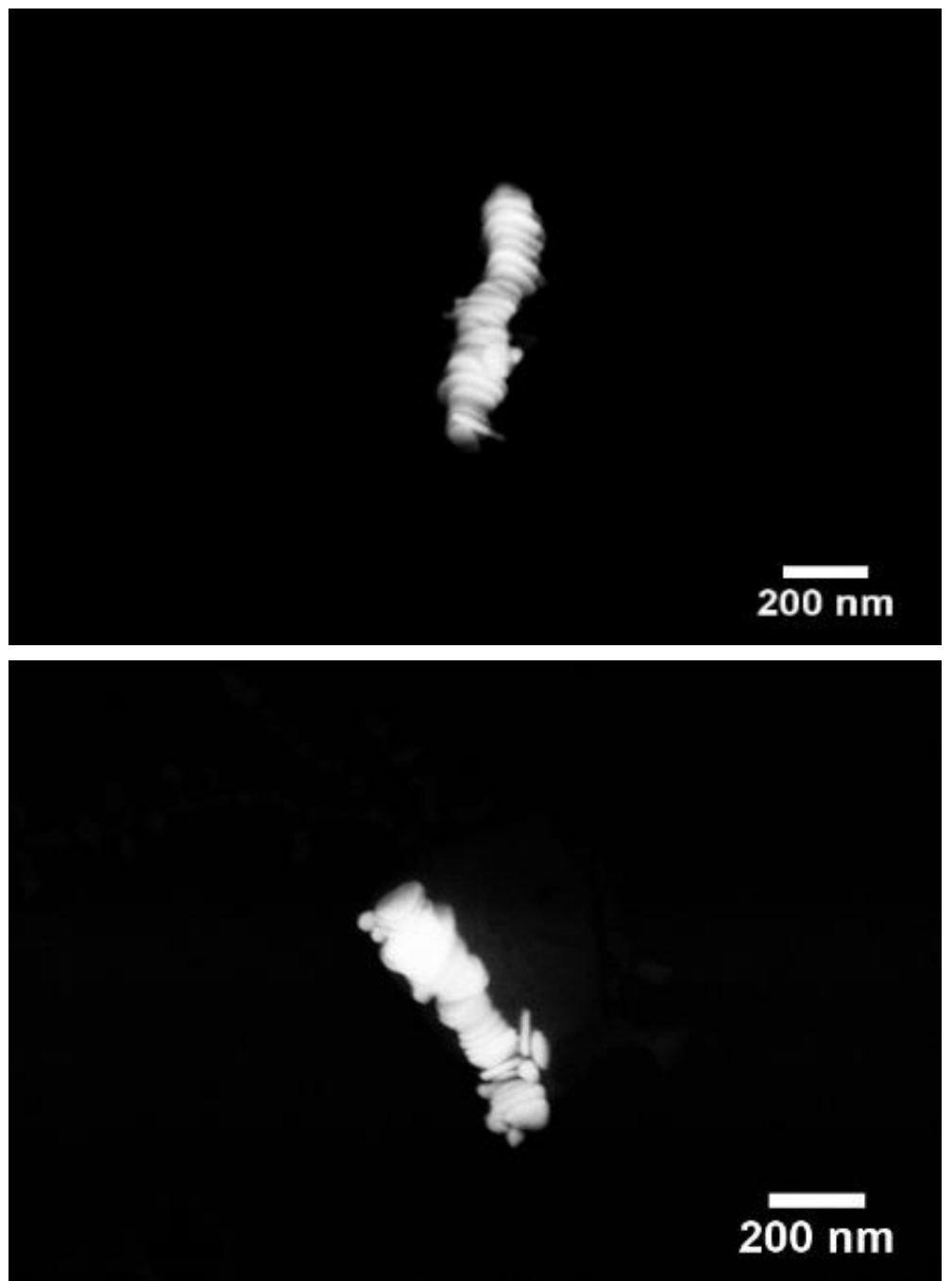

Figure B1: Additional SEM images of 1D face-to-face arrangement of gold nanoprisms. 

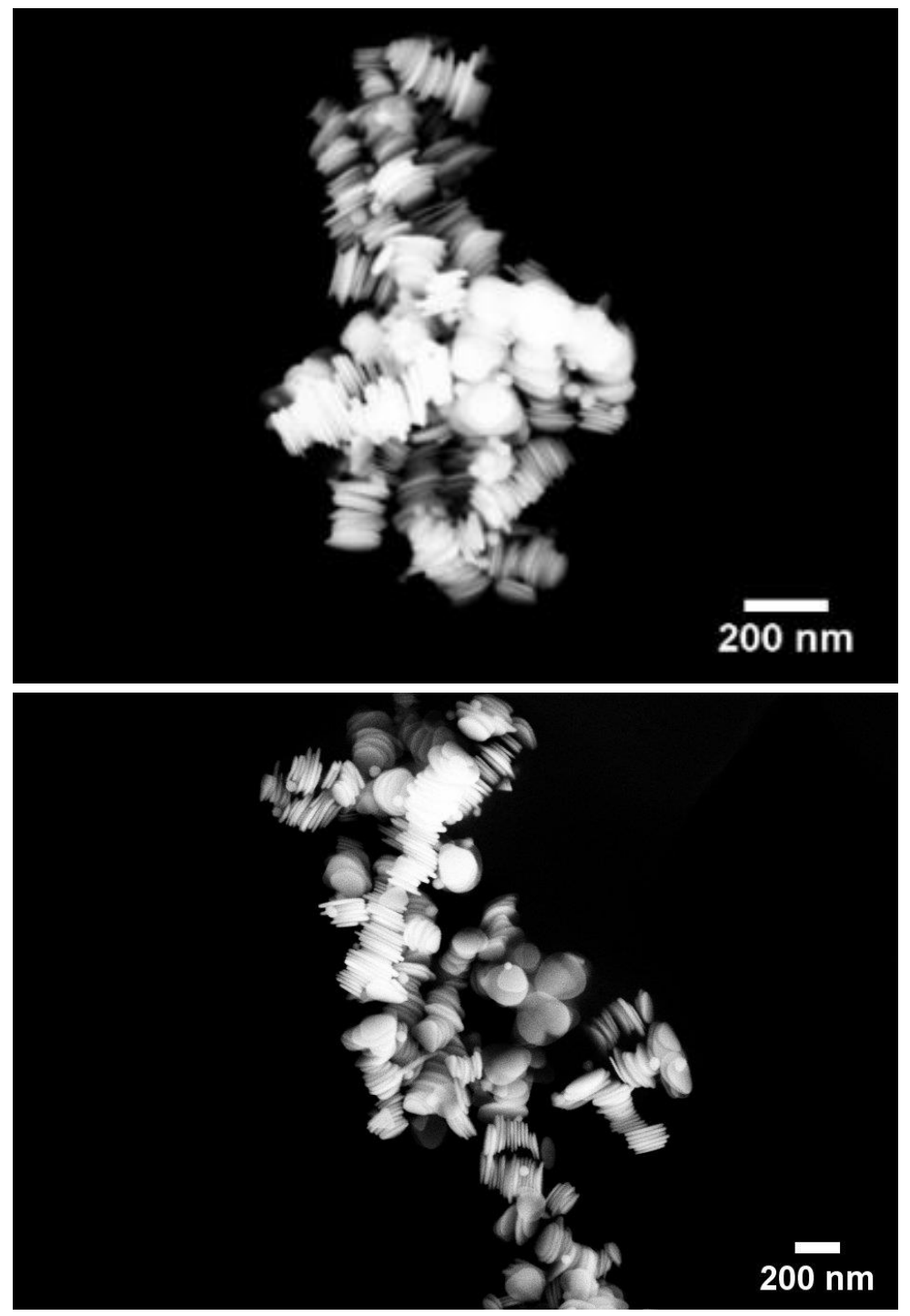

Figure B2: Additional SEM images of 1D face-to-face arrangement of gold nanoprisms. 

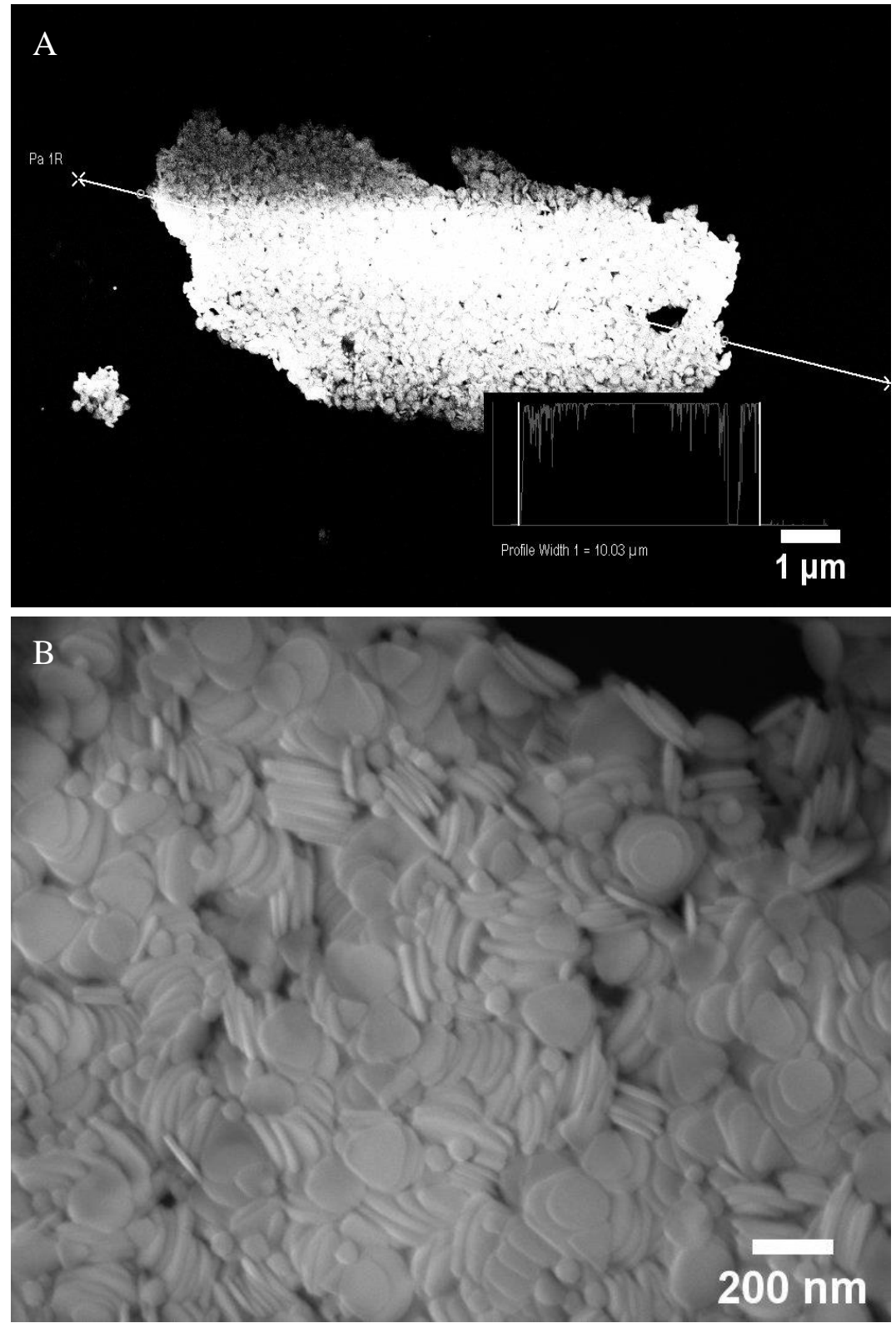

Figure B3: Additional SEM image of 3D hierarchical organization of gold nanoprisms at A) low magnification, and B) high magnification. 

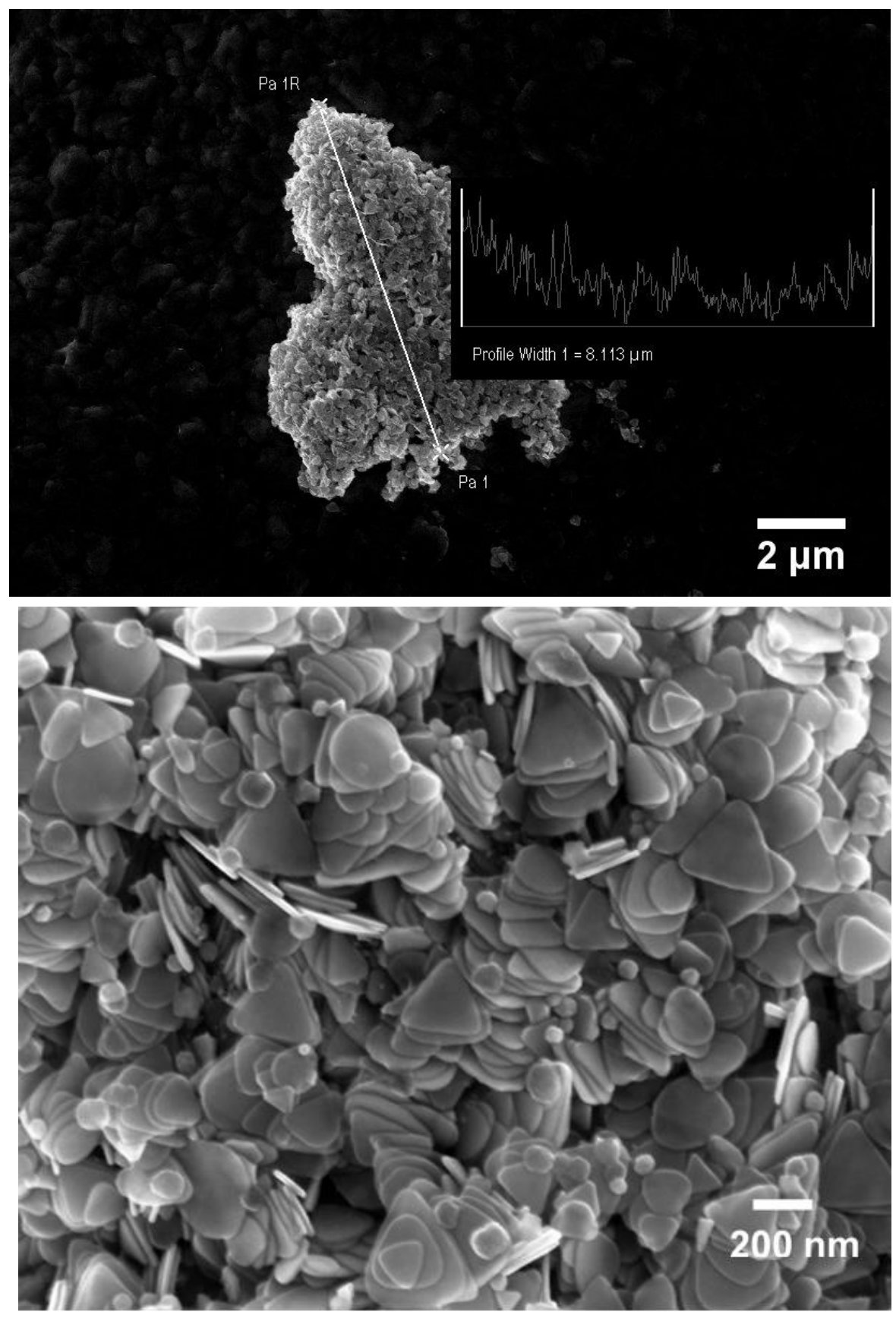

Figure B4: Additional SEM image of 3D hierarchical organization of gold nanoprisms at A) low magnification, and B) high magnification. 

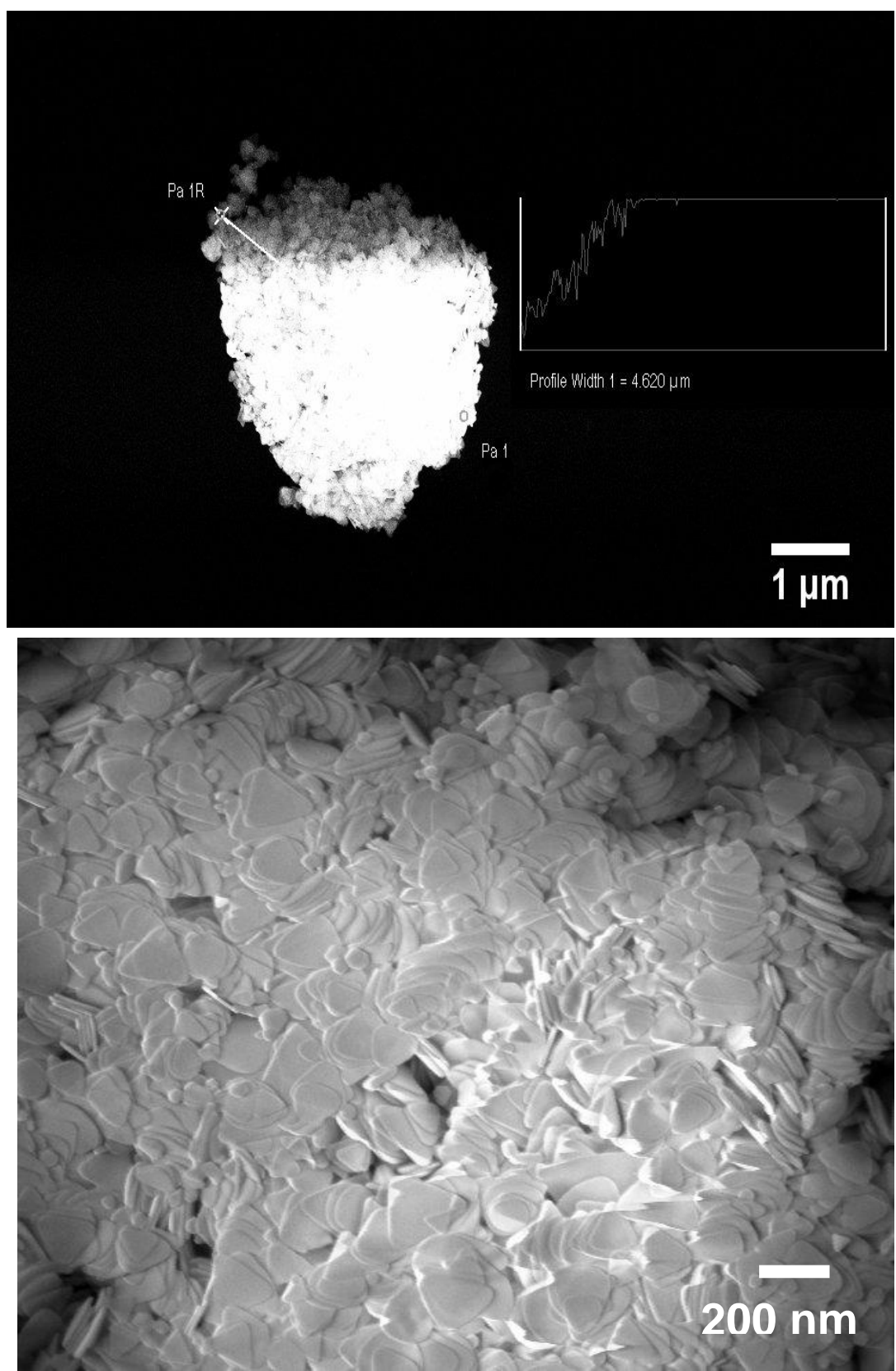

Figure B5: Additional SEM image of 3D hierarchical organization of gold nanoprisms at A) low magnification, and B) high magnification. 

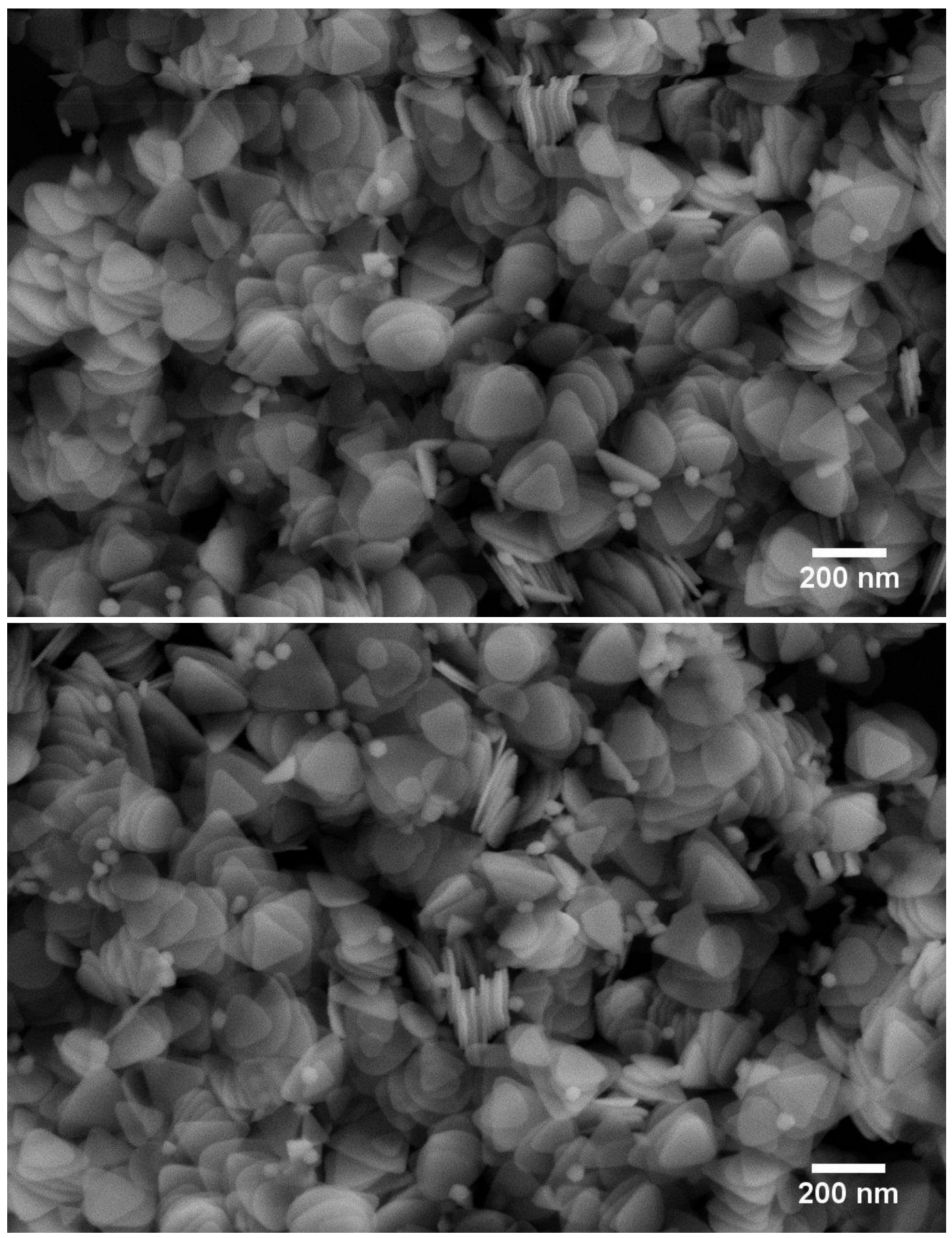

Figure B6: Additional SEM images of 3D hierarchical organization of gold nanoprisms on a glass substrate. 


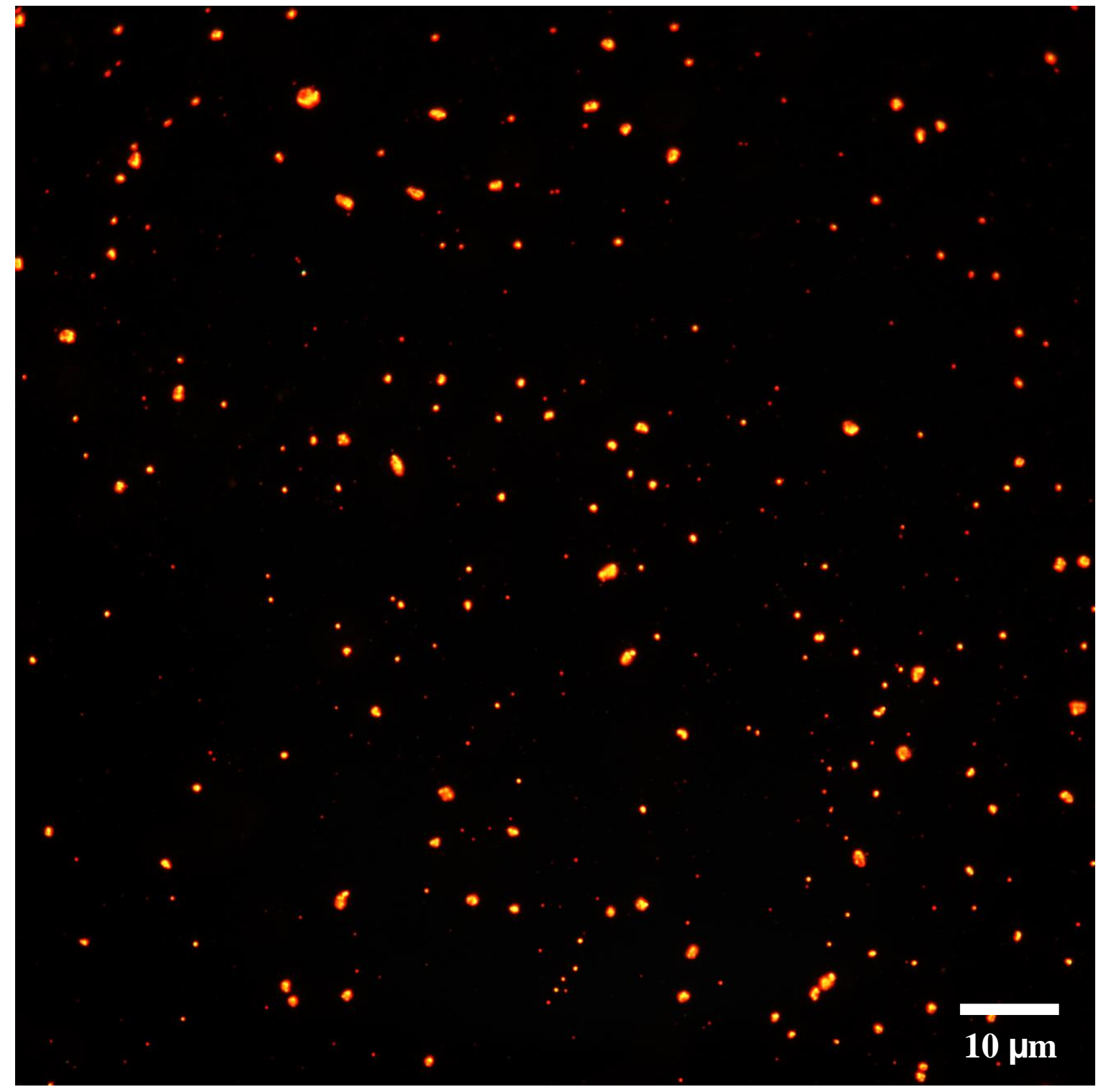

Figure B7: Additional darkfield microscopy image of face-to-face stacking of nanoprisms at room temperature. 


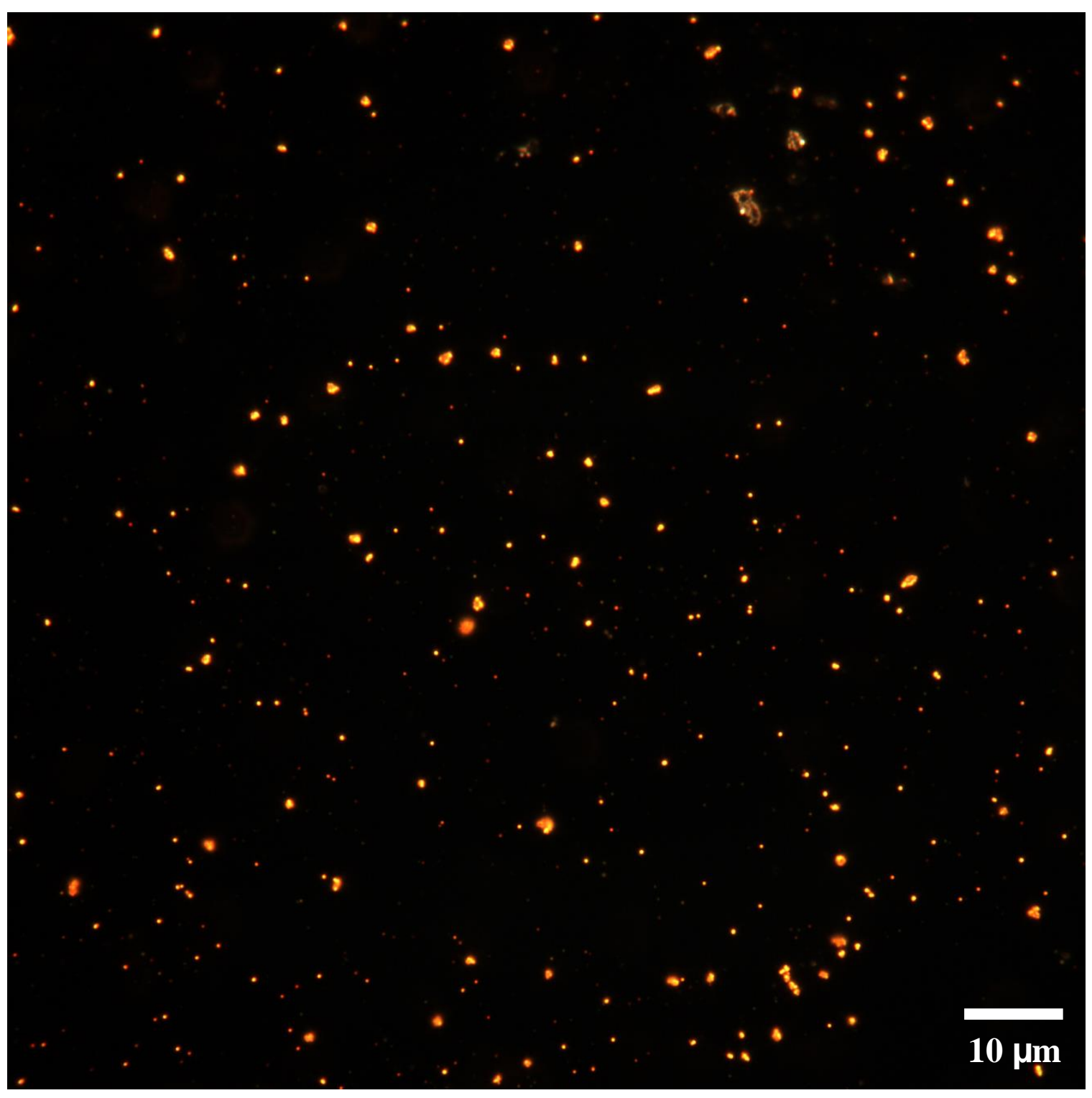

Figure B8: Additional darkfield microscopy image of face-to-face stacking of nanoprisms at room temperature. 


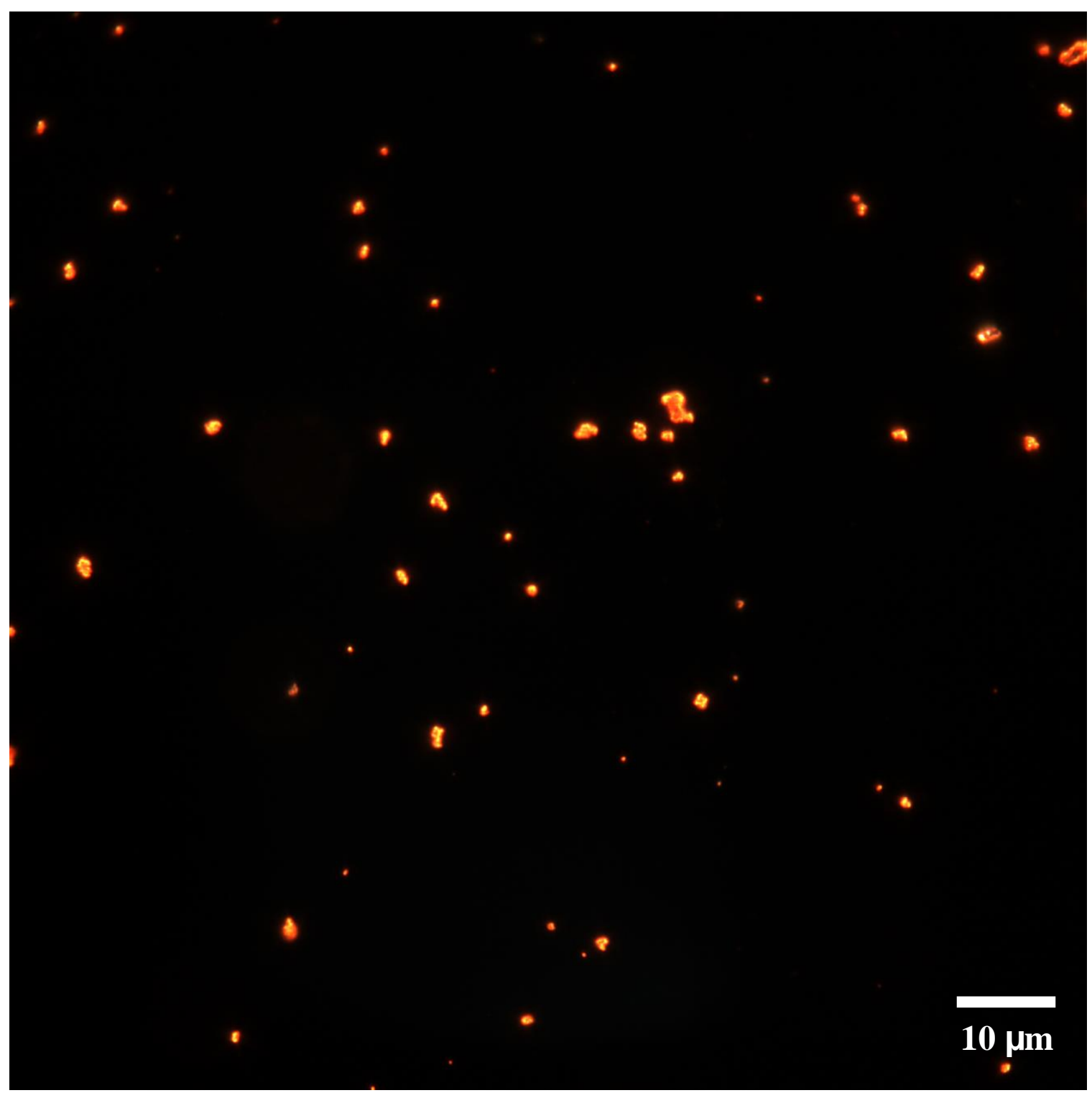

Figure B9: Additional darkfield microscopy image of long-range DNA mediated ordering of nanoprisms at $65^{\circ} \mathrm{C}$. 


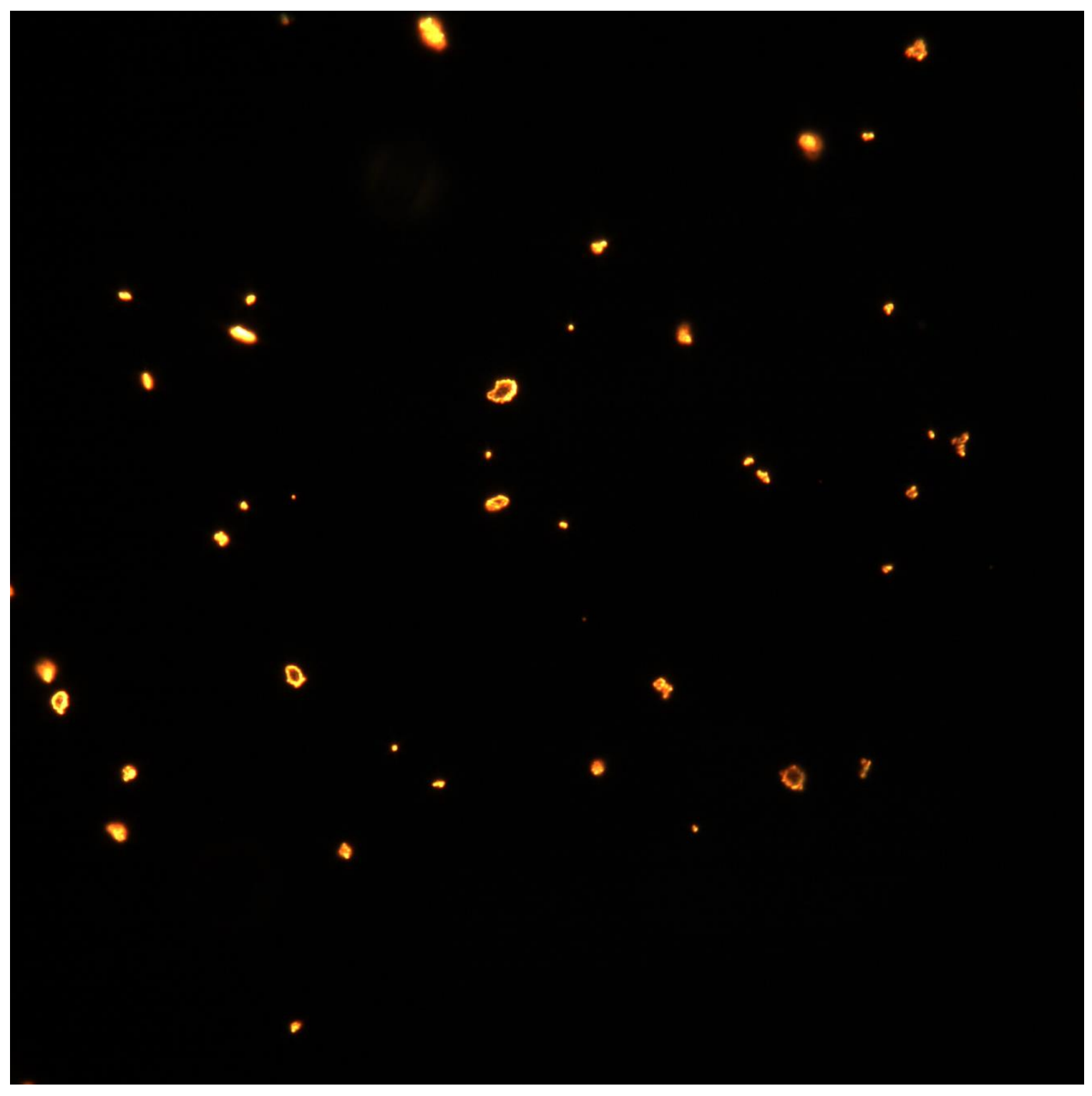

Figure B10: Additional darkfield microscopy image of long-range DNA mediated ordering of nanoprisms at $65^{\circ} \mathrm{C}$. 


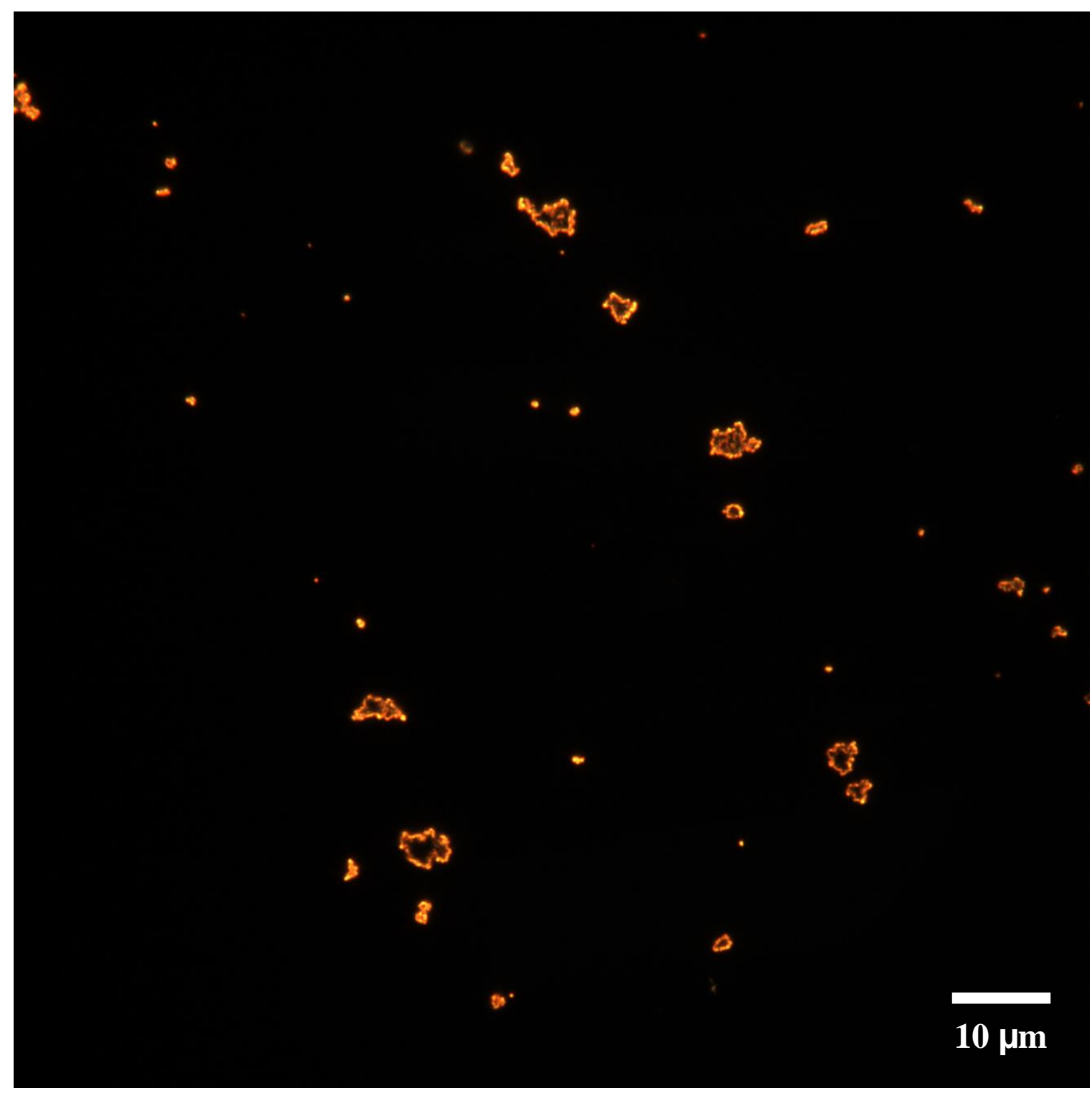

Figure B11: Additional darkfield microscopy image of long-range DNA mediated ordering of nanoprisms at $66^{\circ} \mathrm{C}$. 


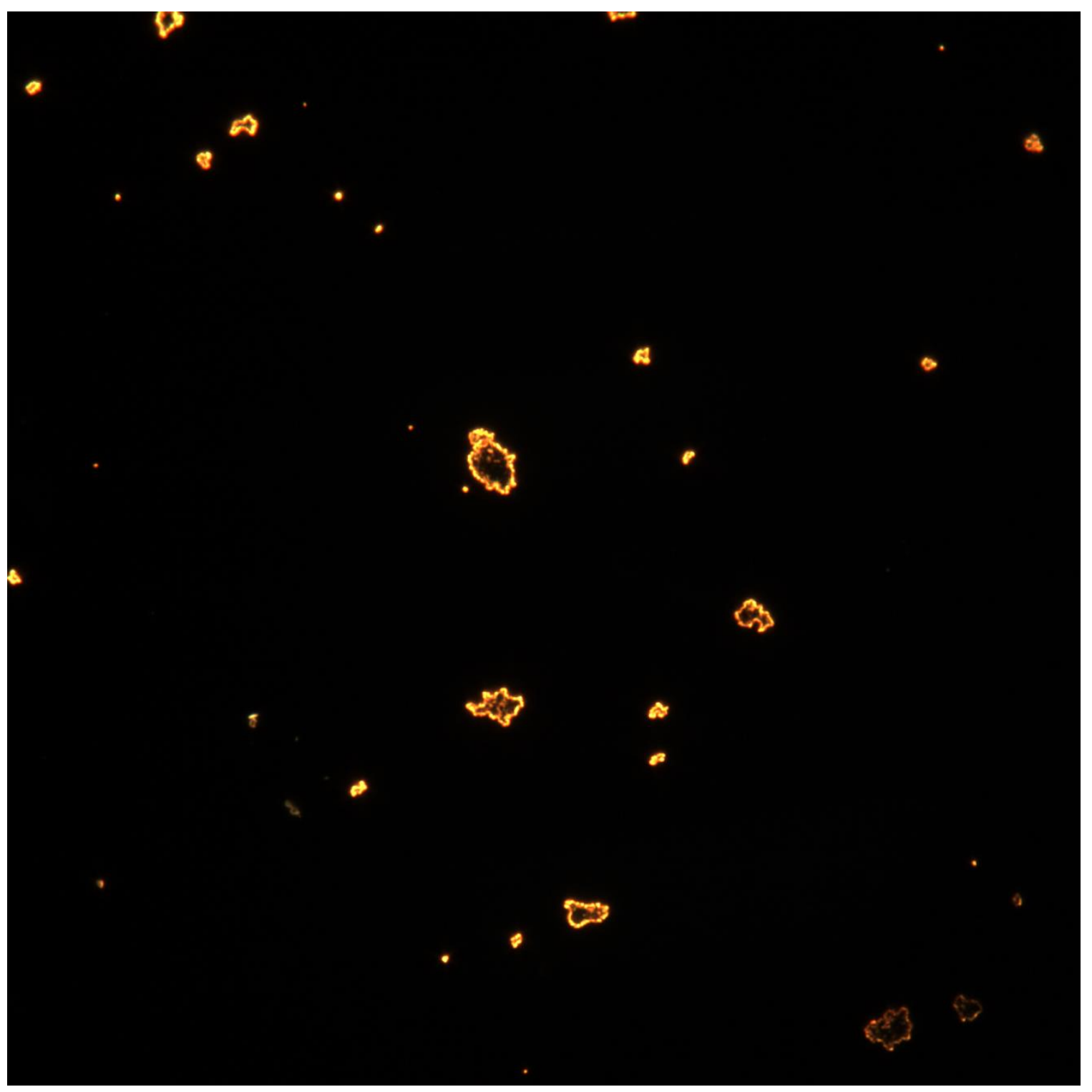

Figure B12: Additional darkfield microscopy image of long-range DNA mediated ordering of nanoprisms at $66^{\circ} \mathrm{C}$. 


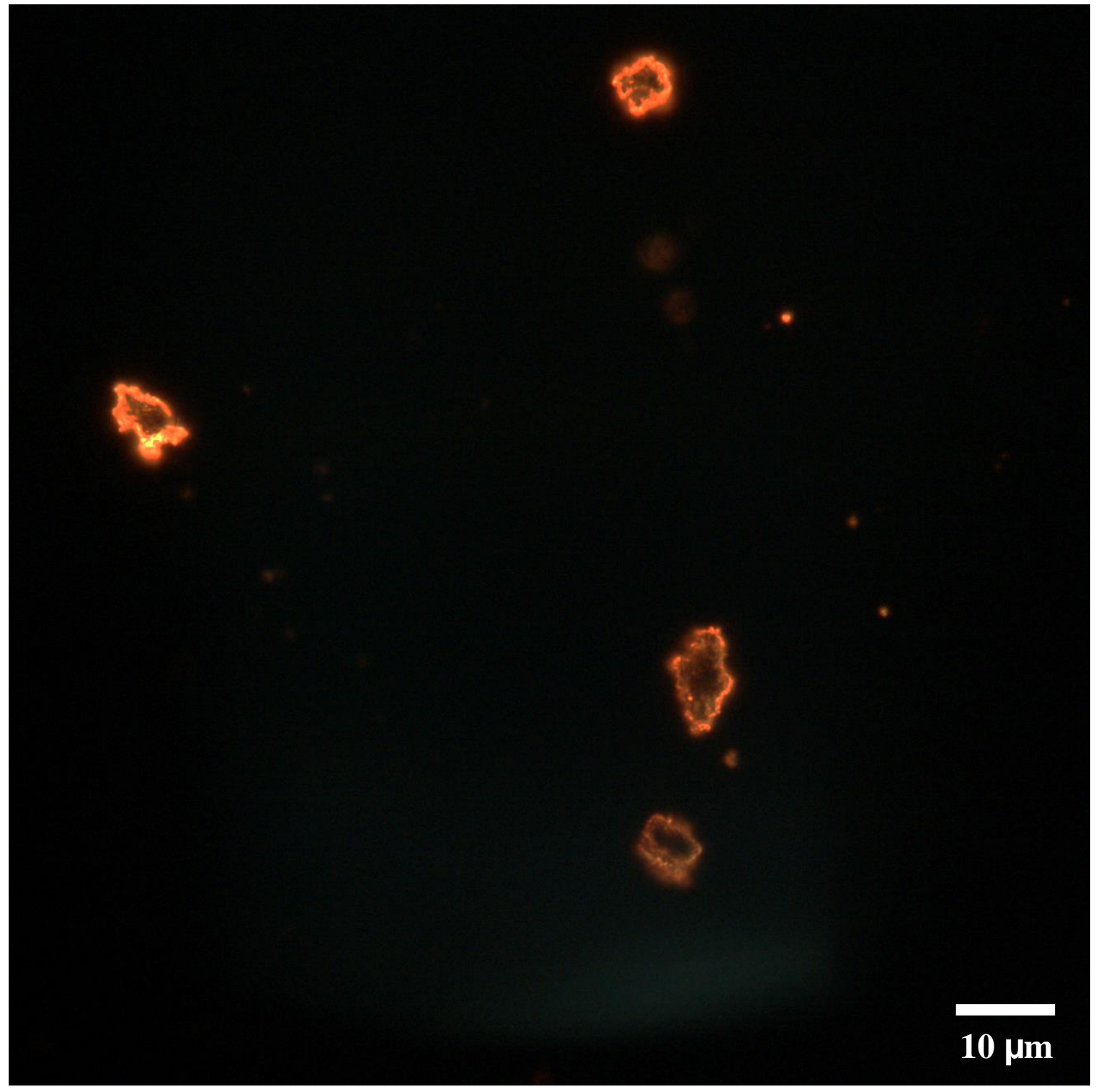

Figure B13: Additional darkfield microscopy image of long-range DNA mediated ordering of nanoprisms at just below the melting temperature of 1D nanoprisms stack $\left(\mathrm{T}_{\mathrm{m}} \sim 68.5^{\circ} \mathrm{C}\right)$ 


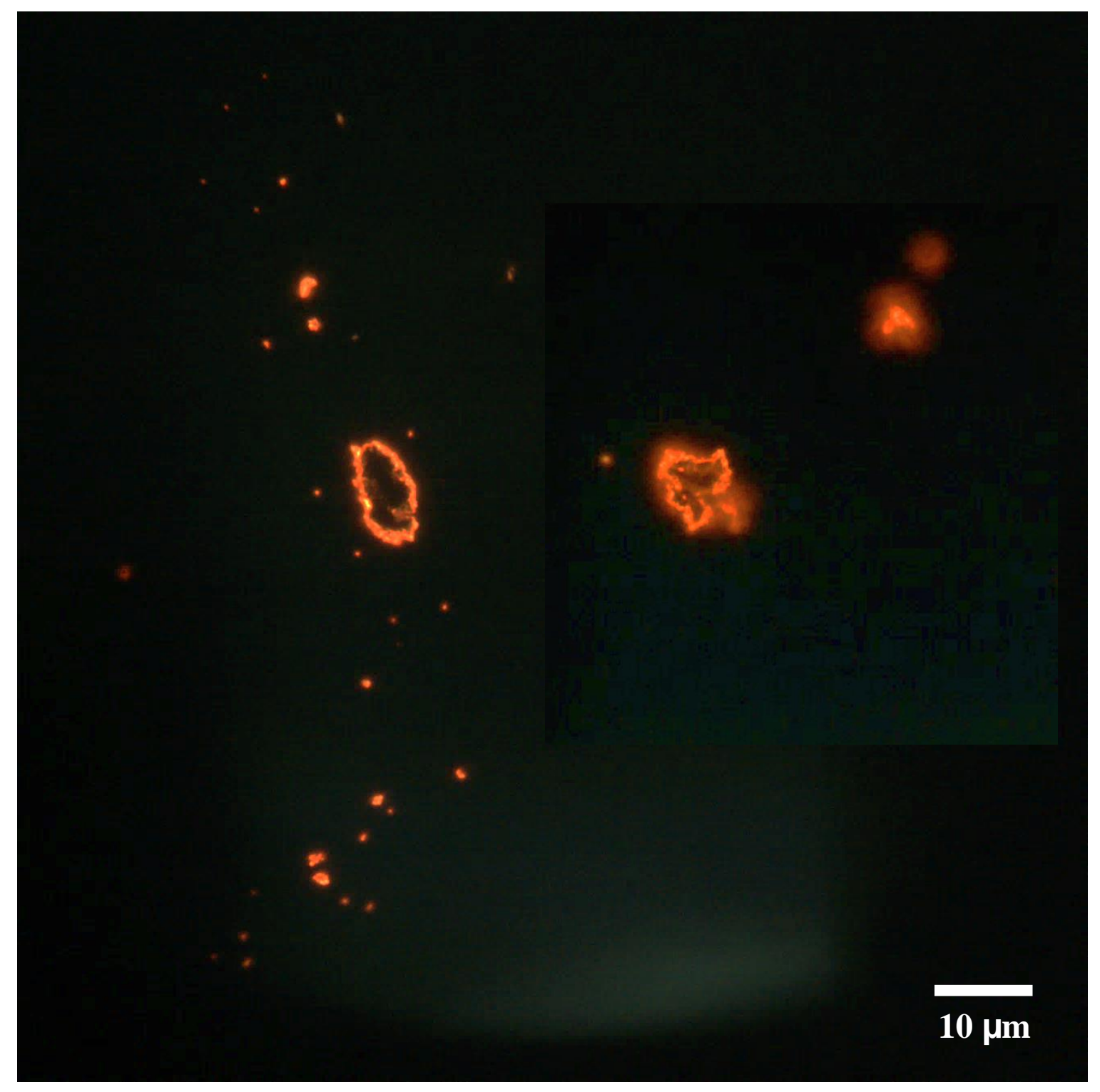

Figure B14: Additional darkfield microscopy image of long-range DNA mediated ordering of nanoprisms at just below the melting temperature of 1D nanoprism stacks $\left(\mathrm{T}_{\mathrm{m}} \sim 68.5^{\circ} \mathrm{C}\right)$. 


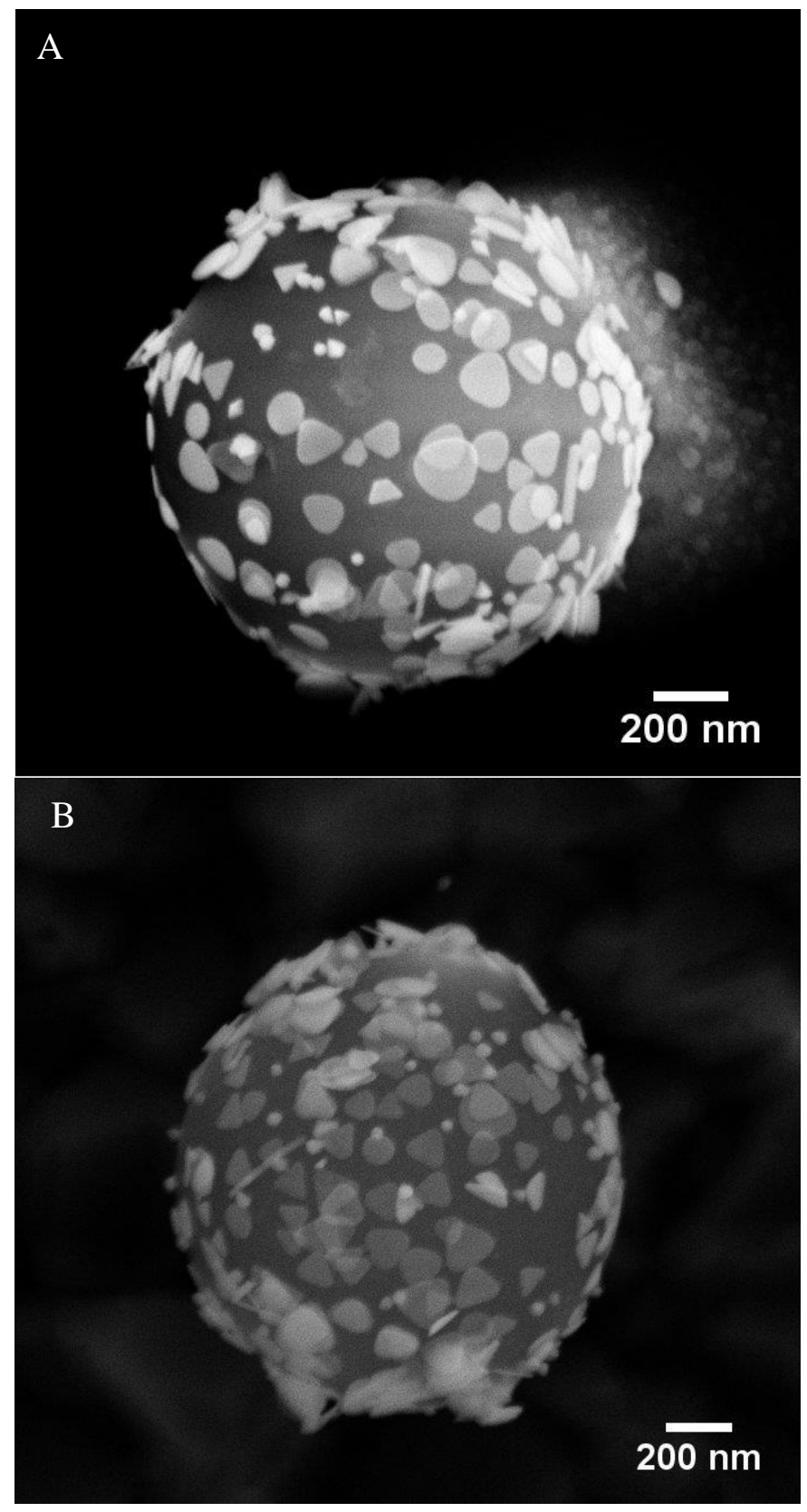

Figure B15: Additional SEM images of PS bead/nanoprism conjugates: A) \& B) Nanoprism loaded single bead. 

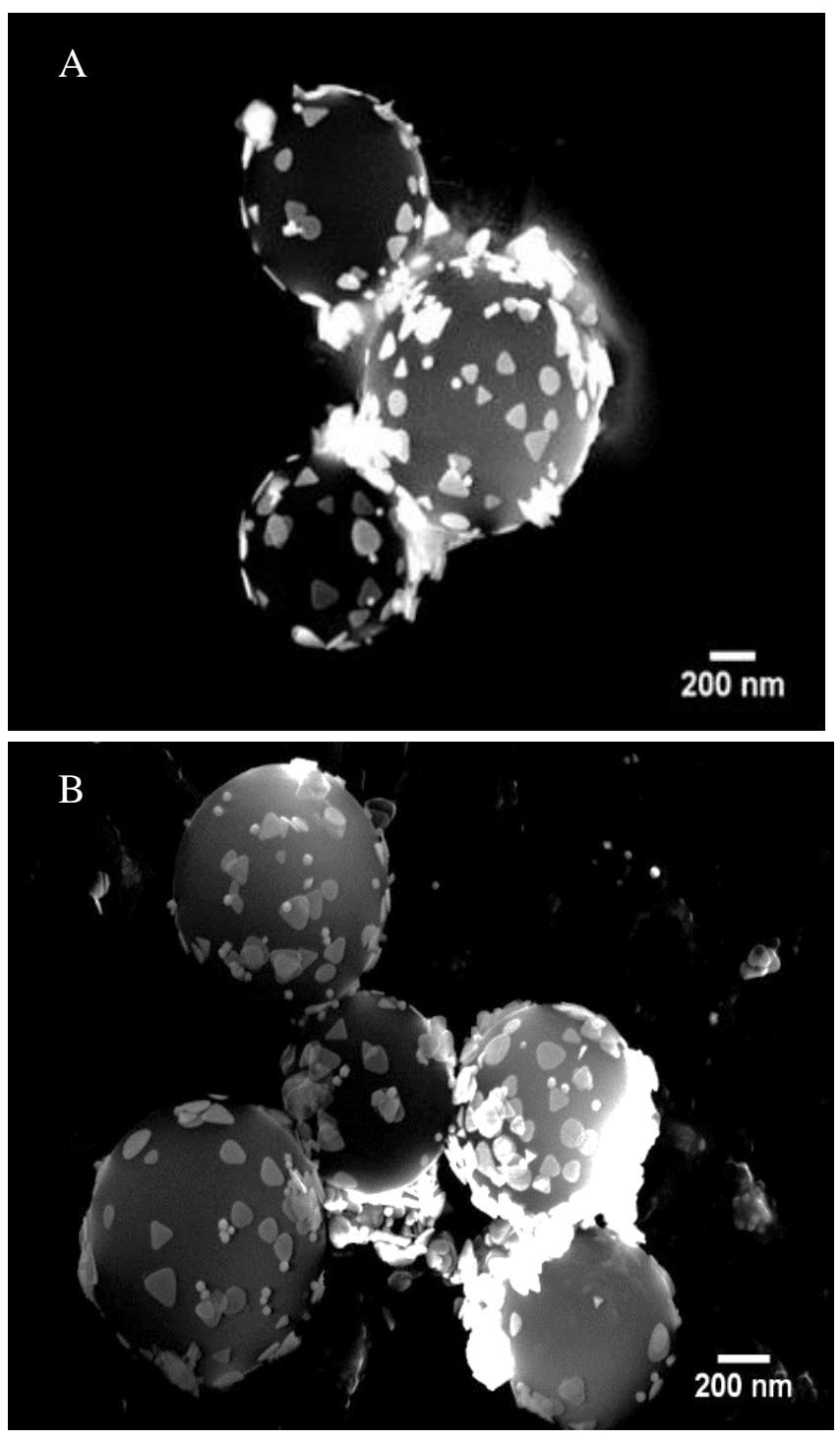

Figure B16: Additional SEM image of PS bead/nanoprism conjugates: A) \& B) PS bead/nanoprism cluster. 

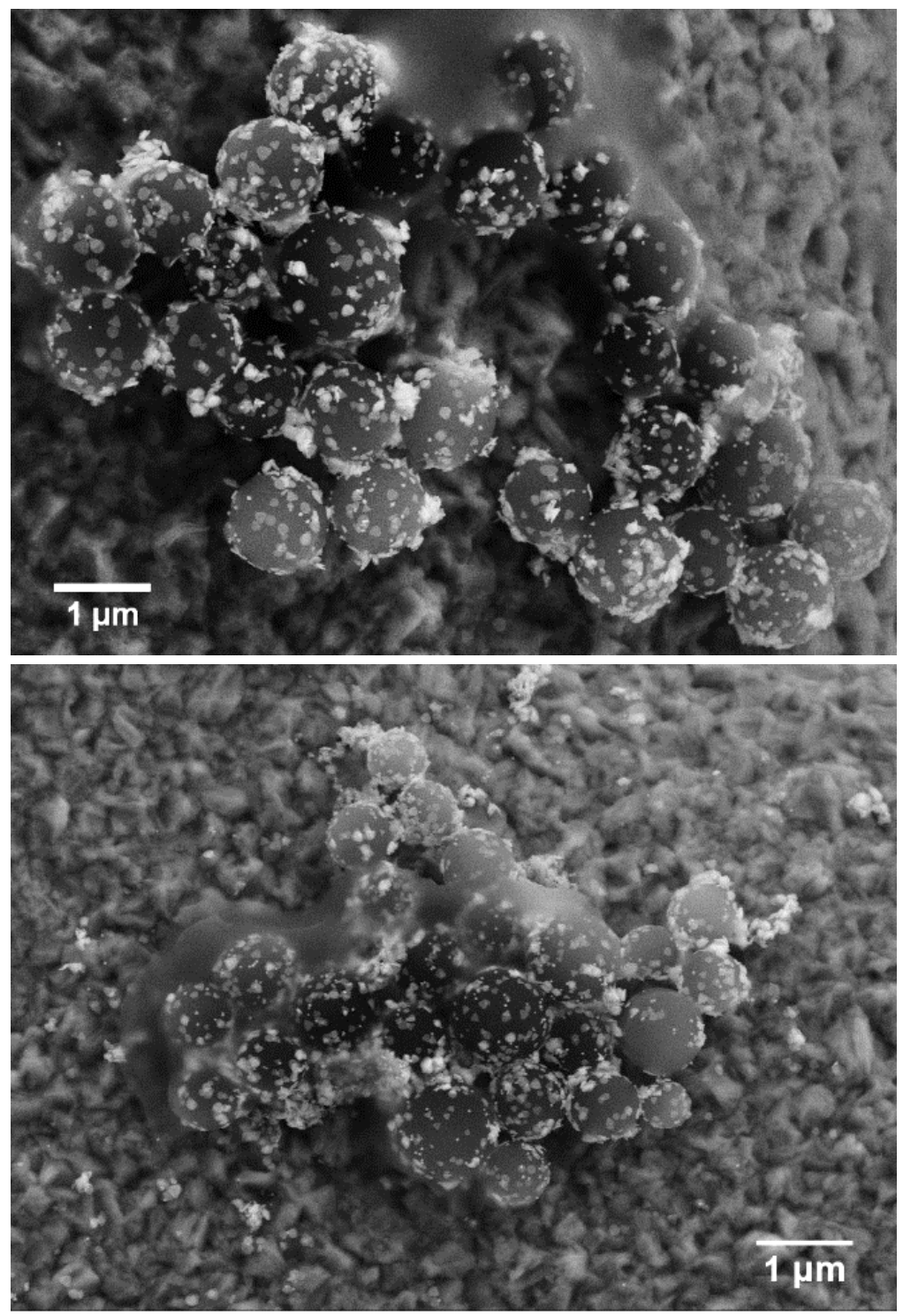

Figure B17: Additional SEM images of 3D PS bead/nanoprism aggregates. 


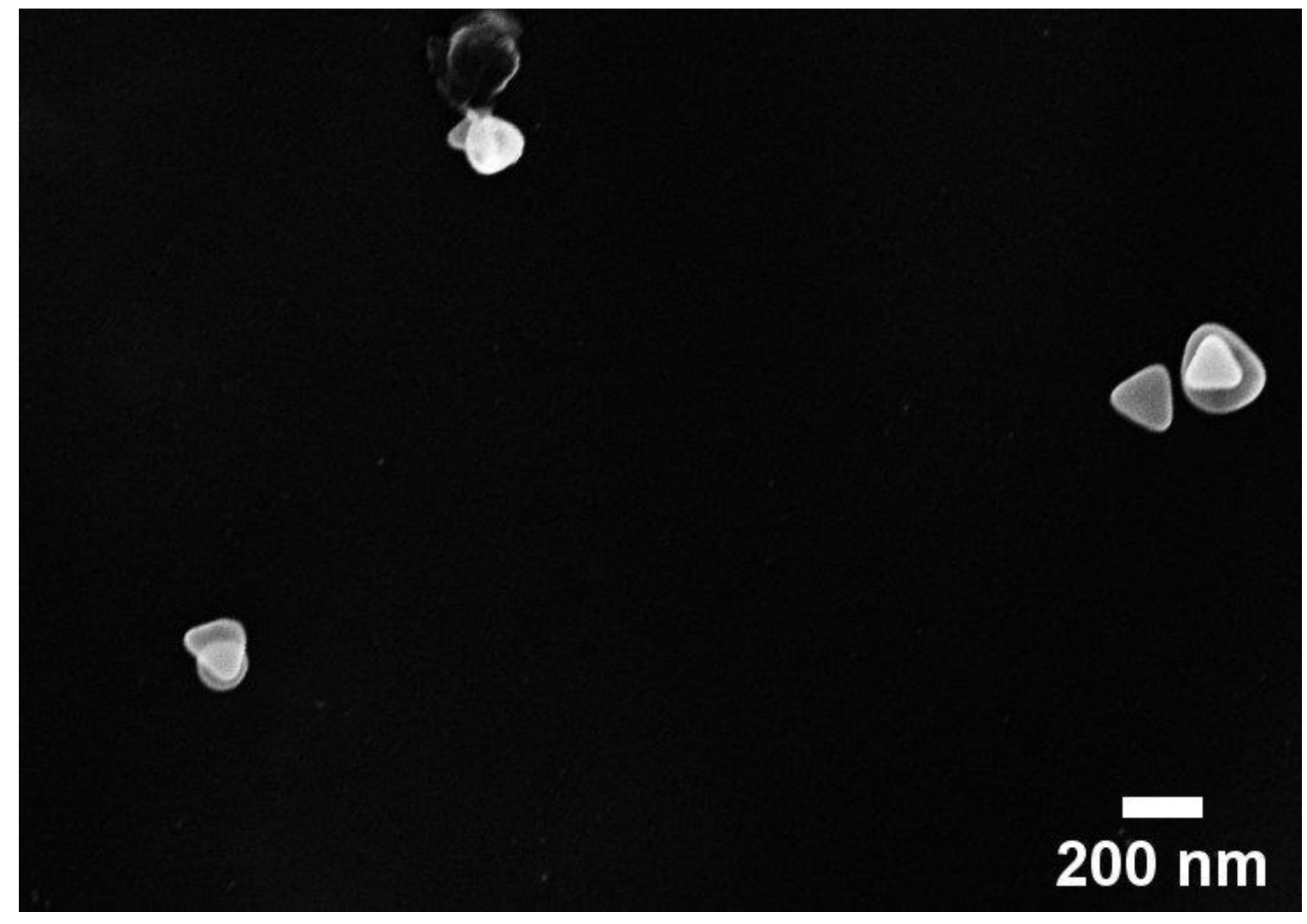

Figure B18: Additional SEM image of gold nanoprism dimers. 


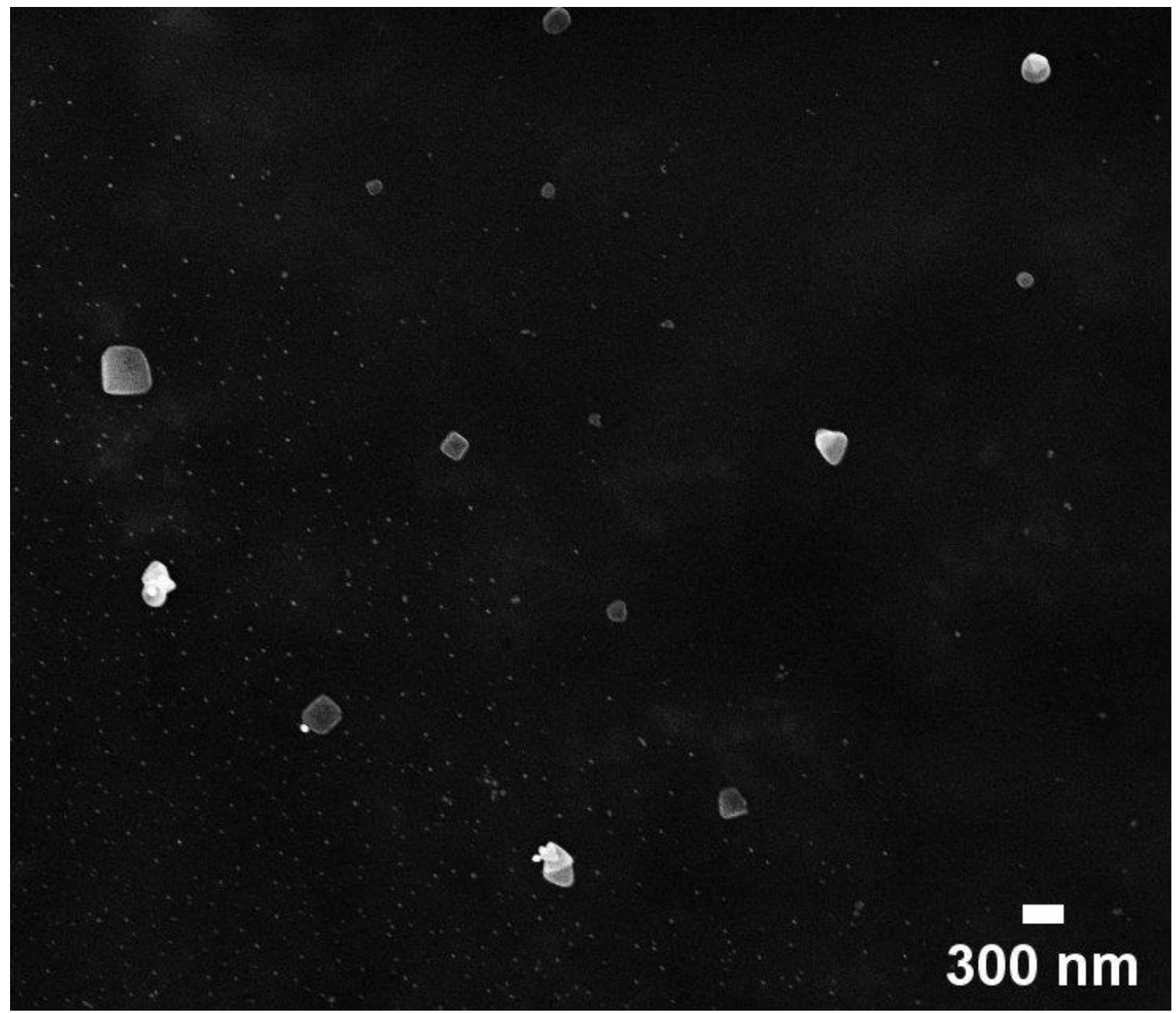

Figure B19: Additional SEM image of gold nanoprism dimers. 

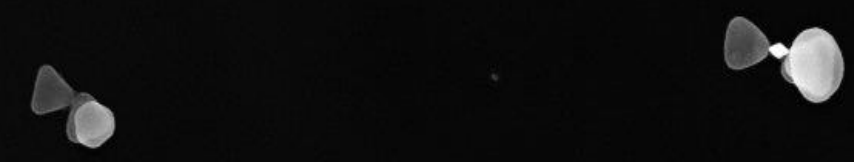

$200 \mathrm{~nm}$

Figure B20: Additional SEM image of gold nanoprism trimers. 


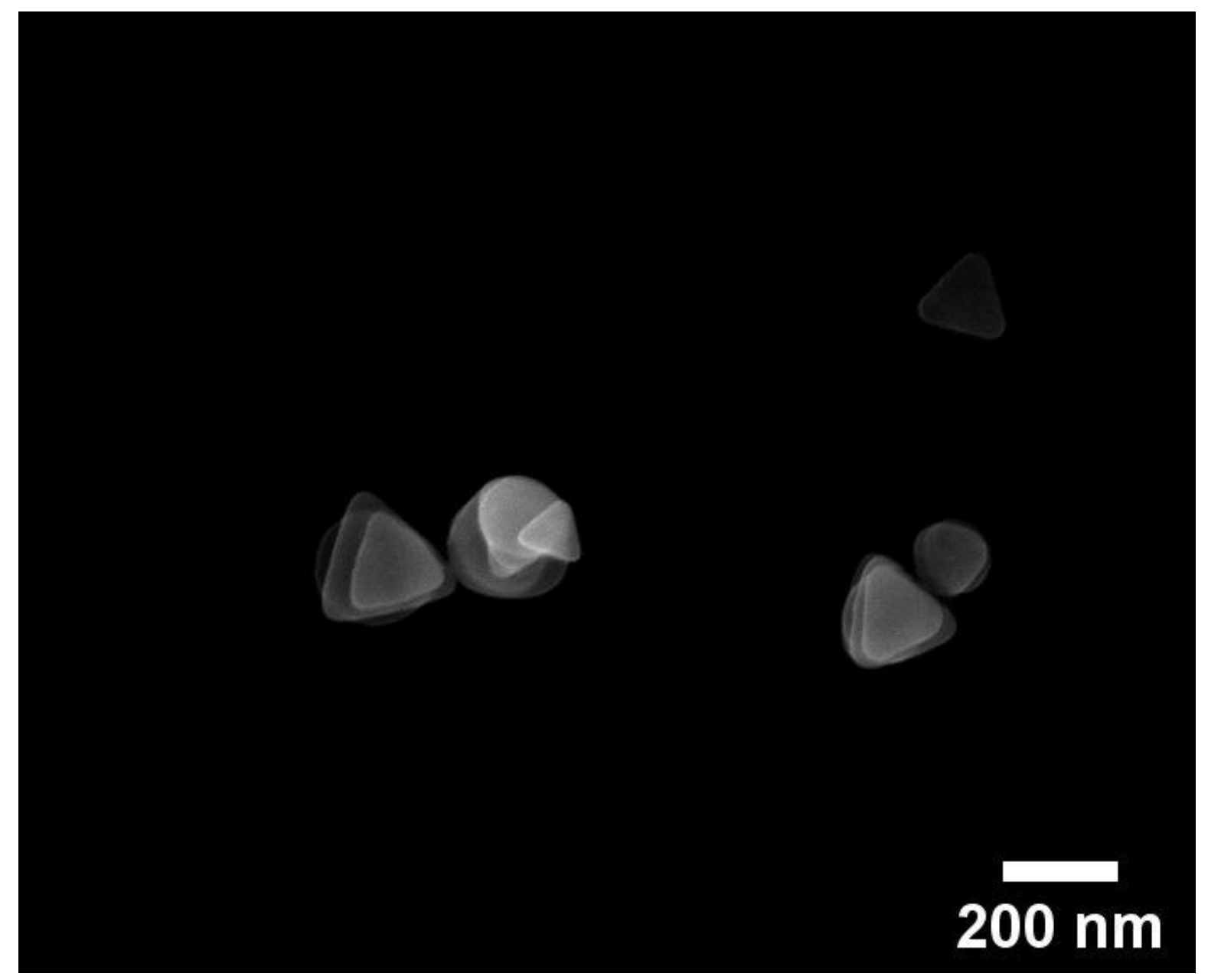

Figure B21: Additional SEM image of gold nanoprism trimers. 


\section{APPENDIX C}

\section{ADDITIONAL SERS SPECTRA OF METHYLENE BLUE AND PL SPECTRA OF ALEXA FLUOR PHALLOIDIN 647 ON 3D NANOPRISMS SUPERLATTICE AND 3D PS BEADS/ NANOPRISMS AGGREGATES}




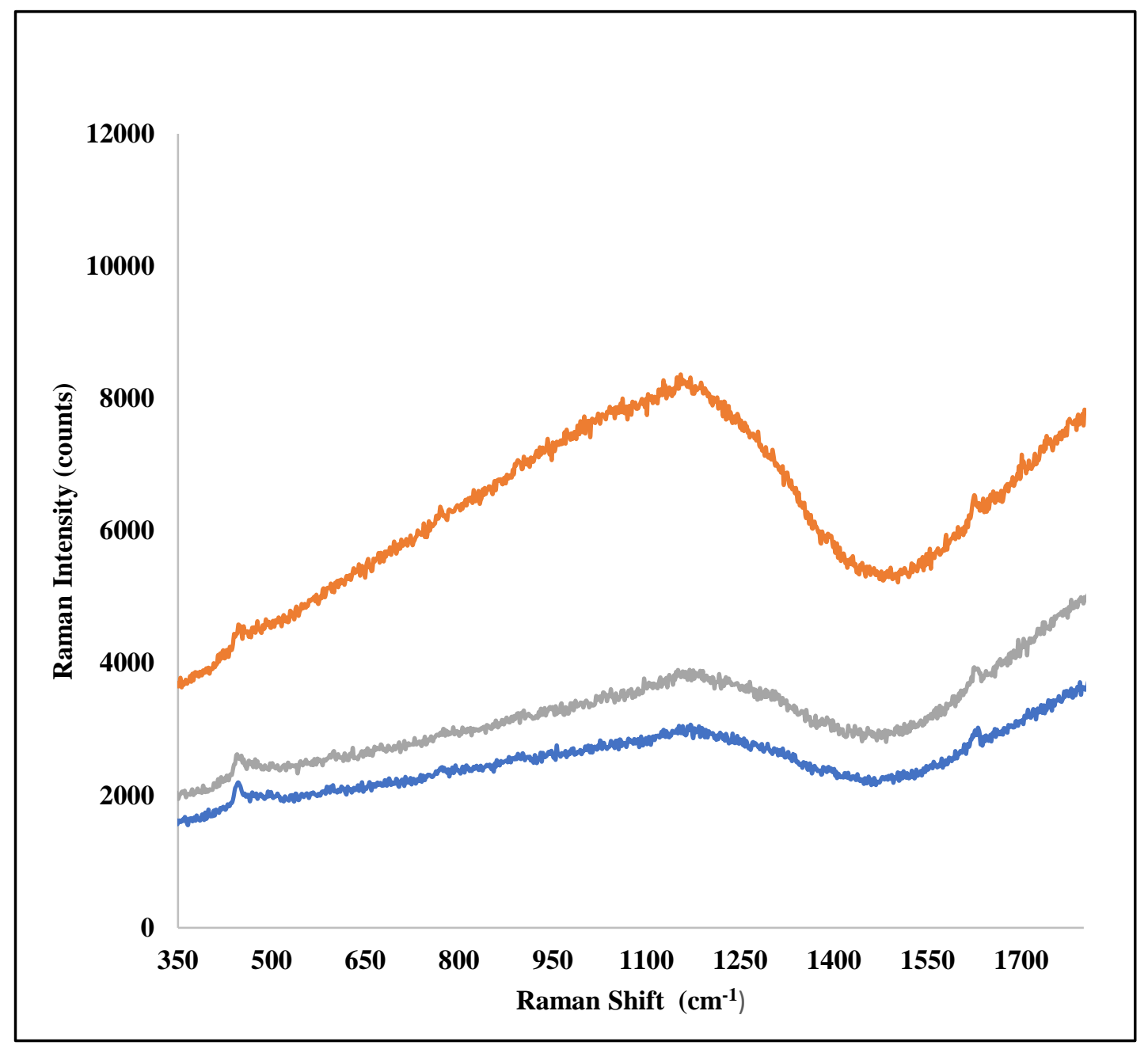

Figure C1: Additional Raman spectra of $10^{-4} \mathrm{M}$ MB on pristine glass slides. 


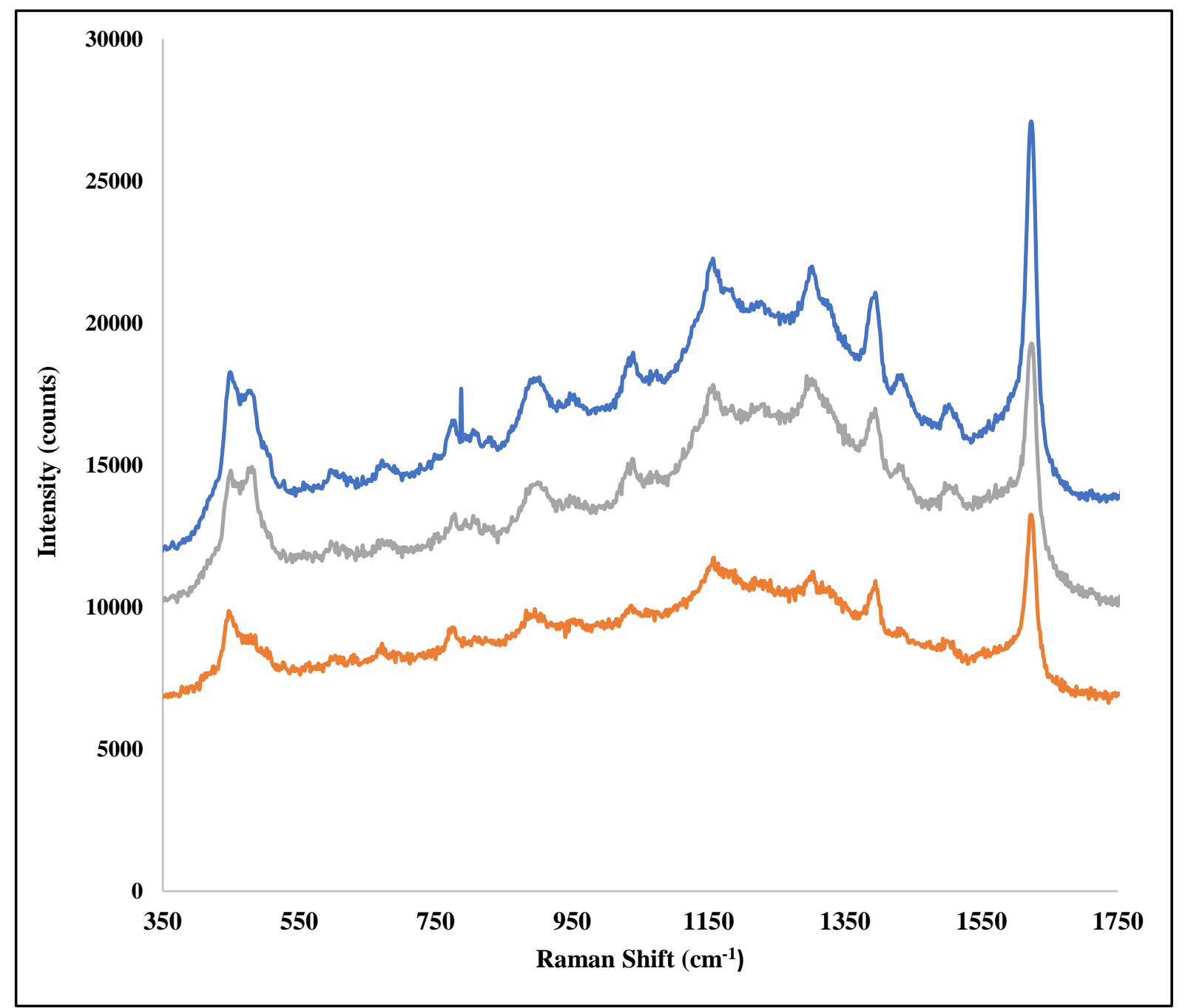

Figure C2: Additional SERS spectra of $10^{-6} \mathrm{M} \mathrm{MB}$ on 3D nanoprism superlattices. 


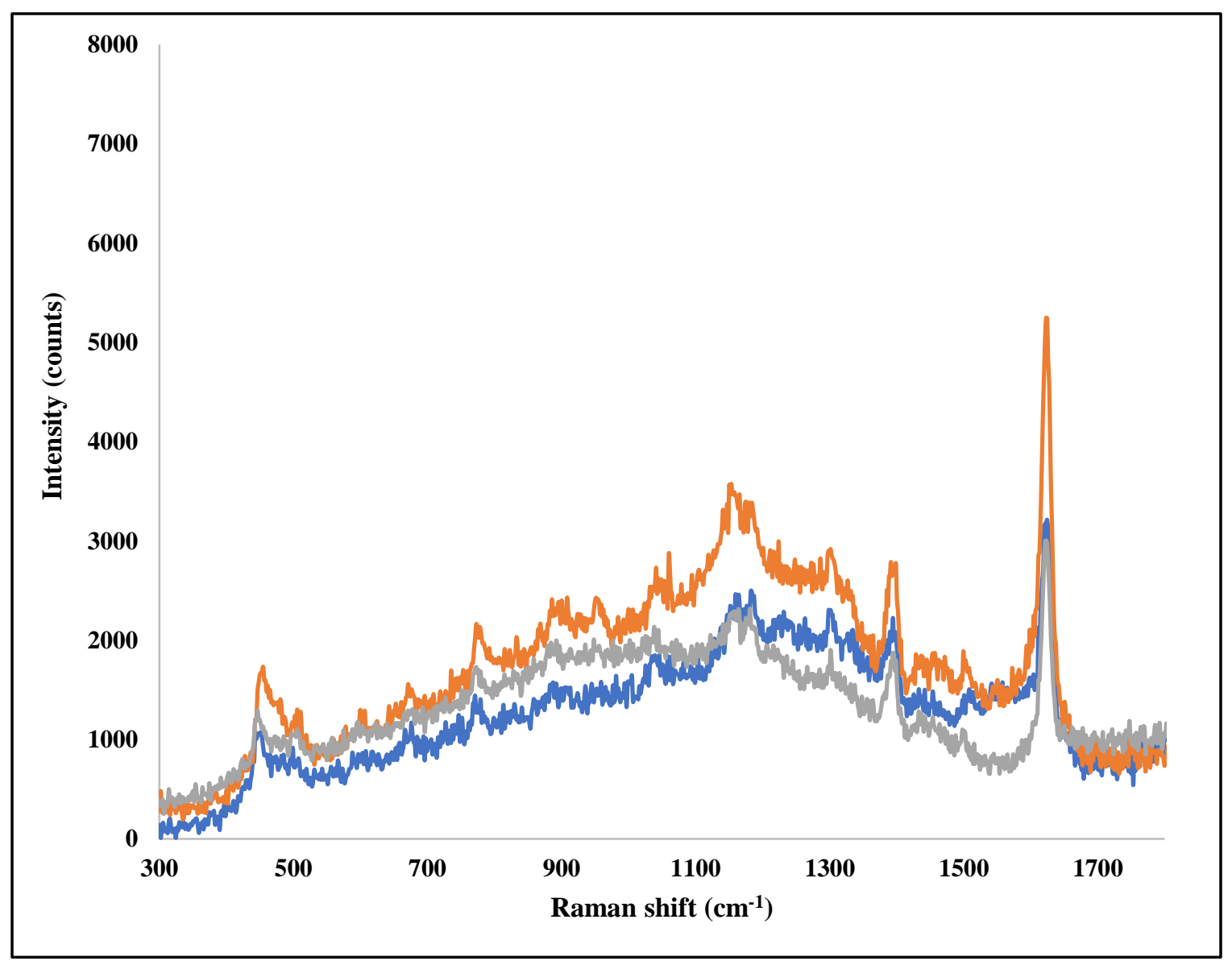

Figure C3: Additional SERS spectra of $10^{-7} \mathrm{M} \mathrm{MB}$ on 3D nanoprism superlattices. 


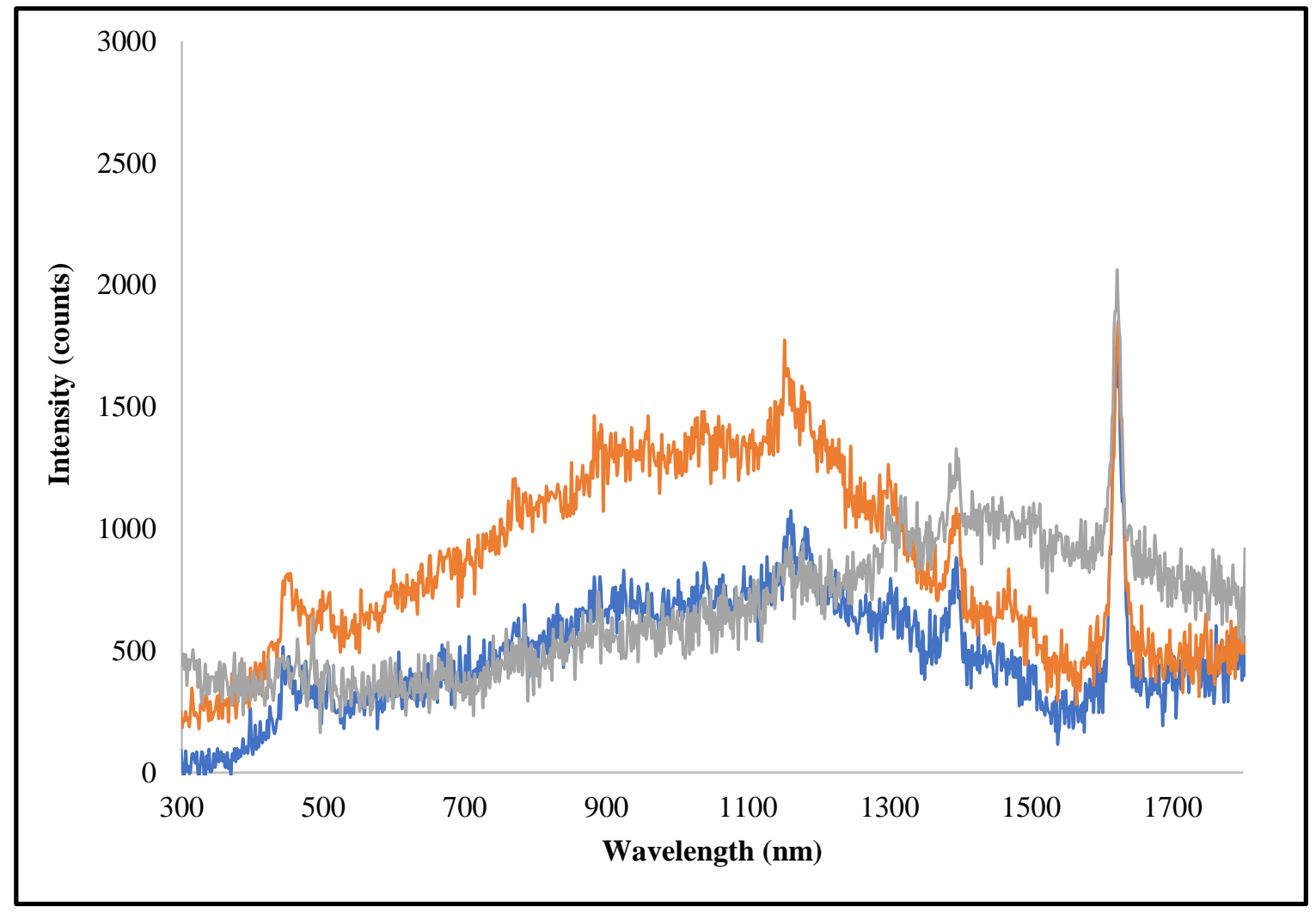

Figure C4: Additional SERS spectra of $10^{-8} \mathrm{M}$ MB on 3D nanoprism superlattices. 


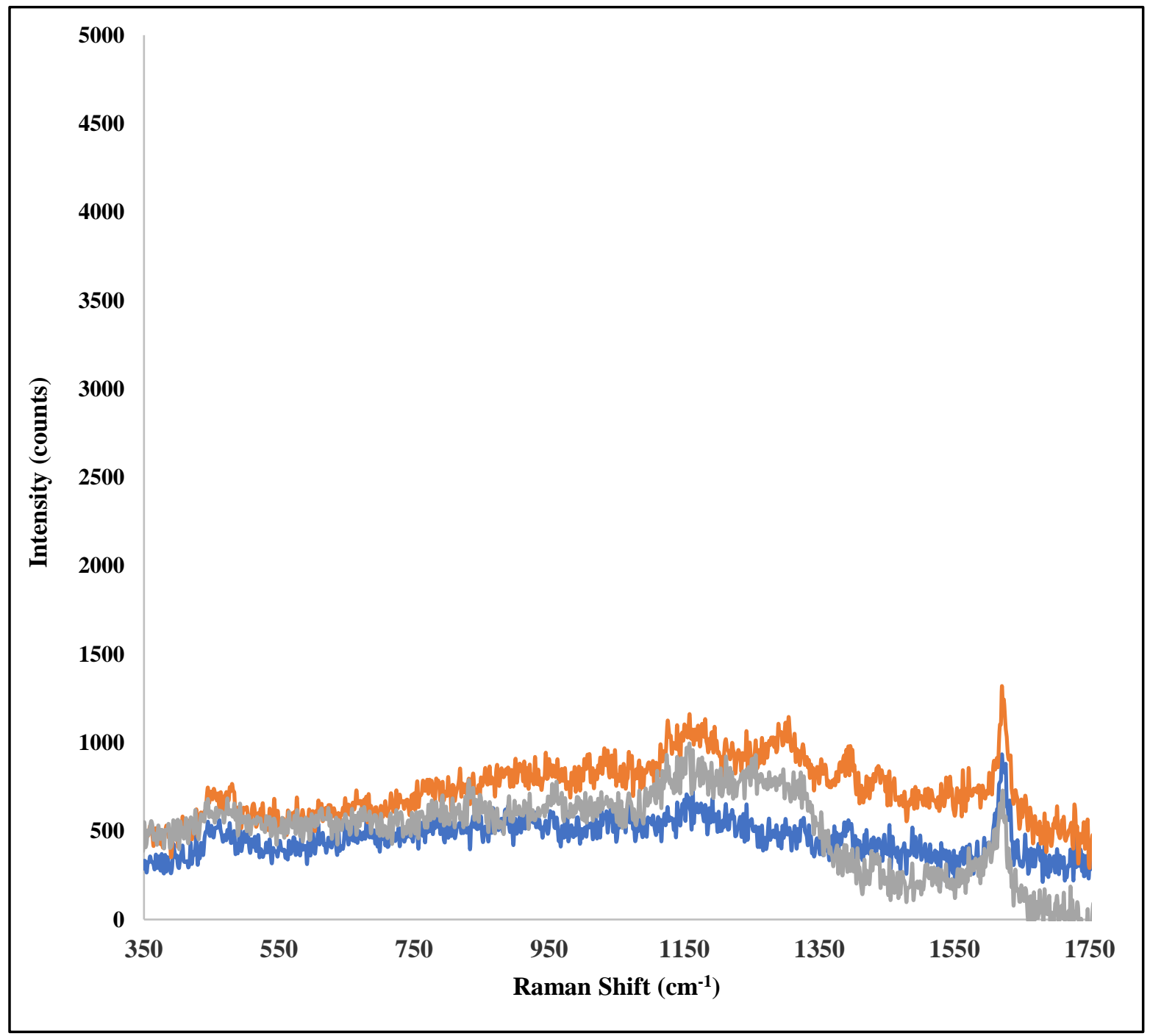

Figure C5: Additional SERS spectra of $10^{-9} \mathrm{M} \mathrm{MB}$ on 3D nanoprism superlattices. 


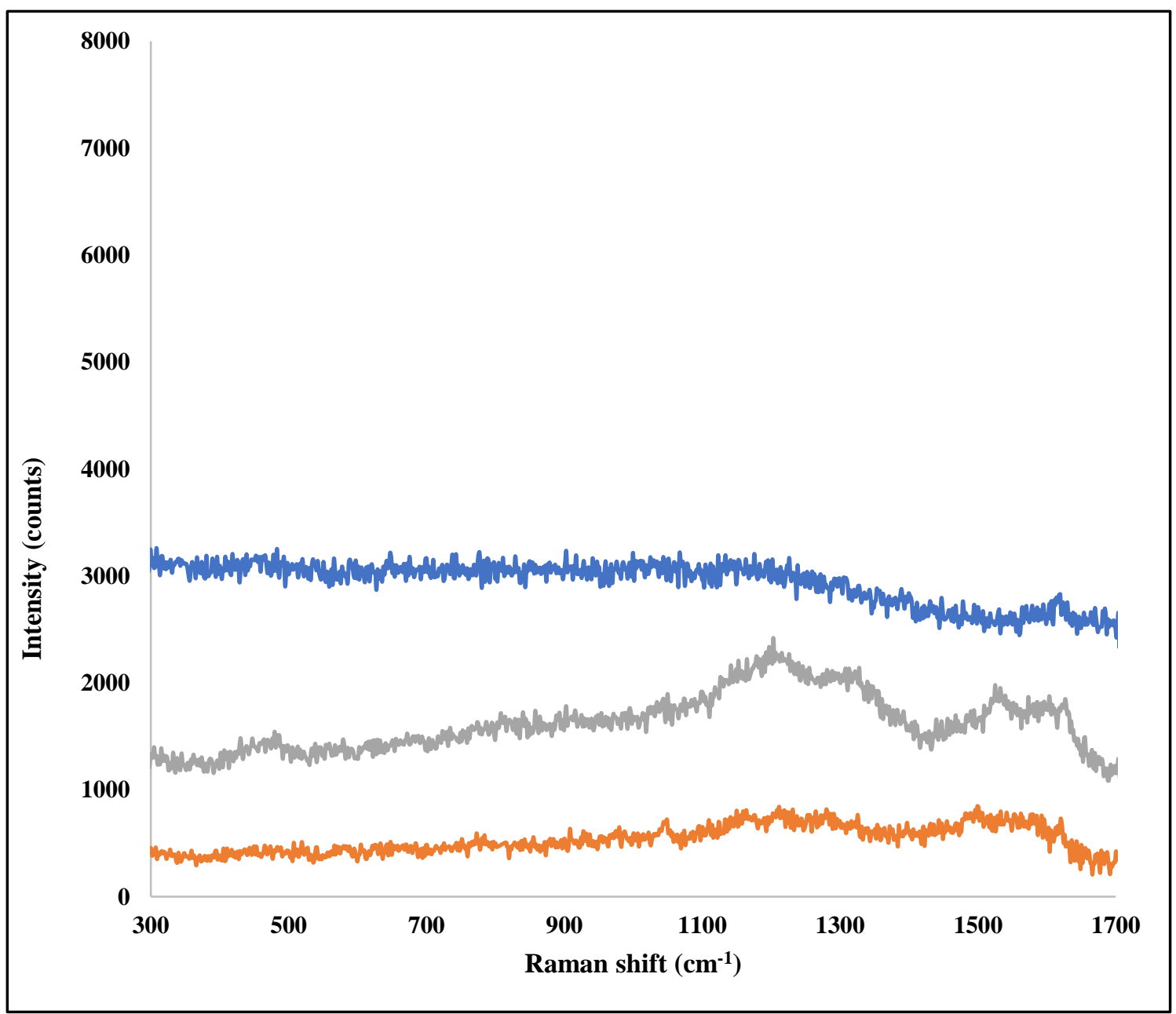

Figure C6: Additional SERS spectra of $10^{-10} \mathrm{M} \mathrm{MB}$ on 3D nanoprism superlattices. 


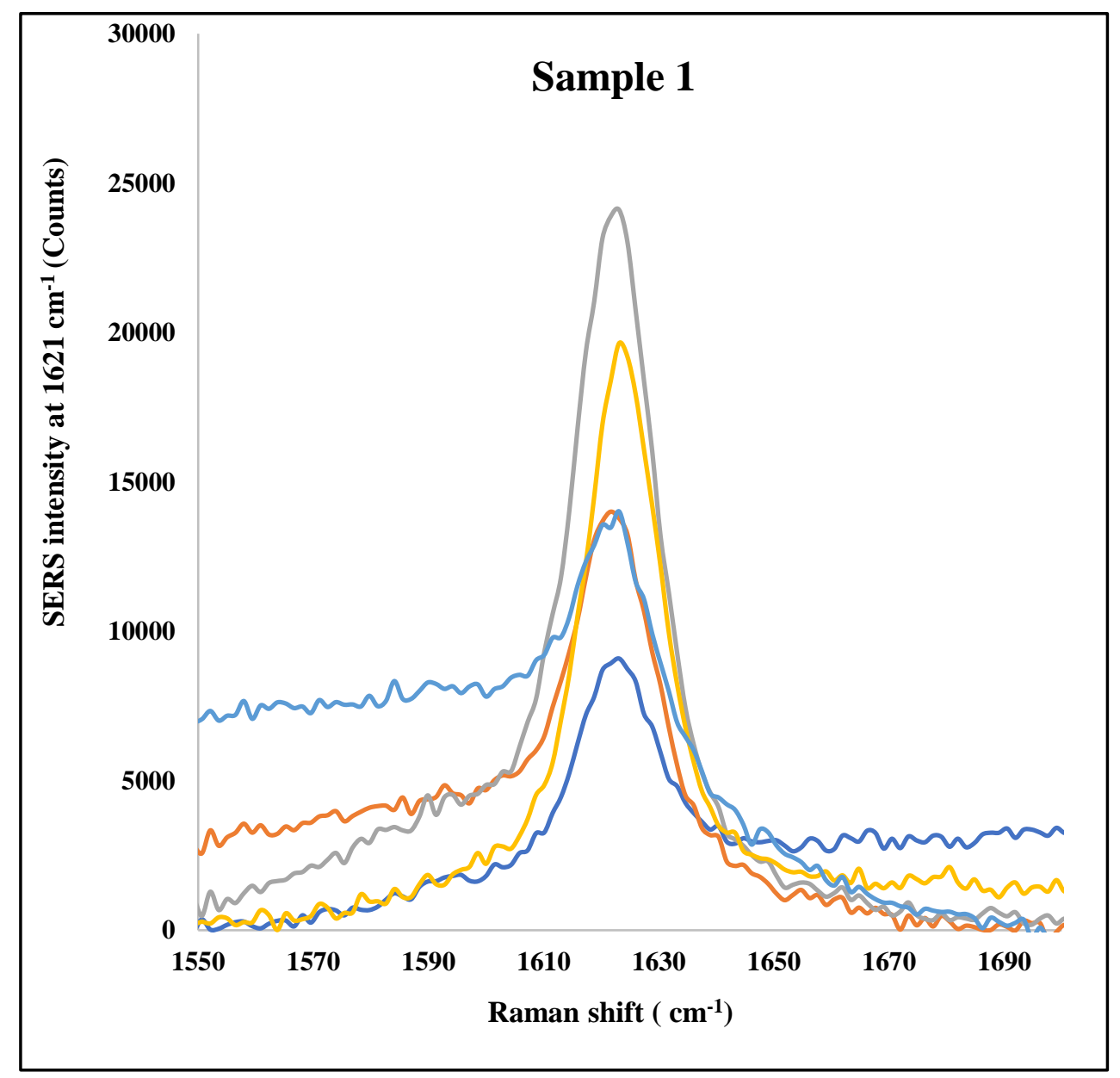

Figure C7: SERS intensity of $10^{-4} \mathrm{M} \mathrm{MB}$ at $1621 \mathrm{~cm}^{-1}$ from 5 different spots of 3D nanoprism superlattices (sample 1). 


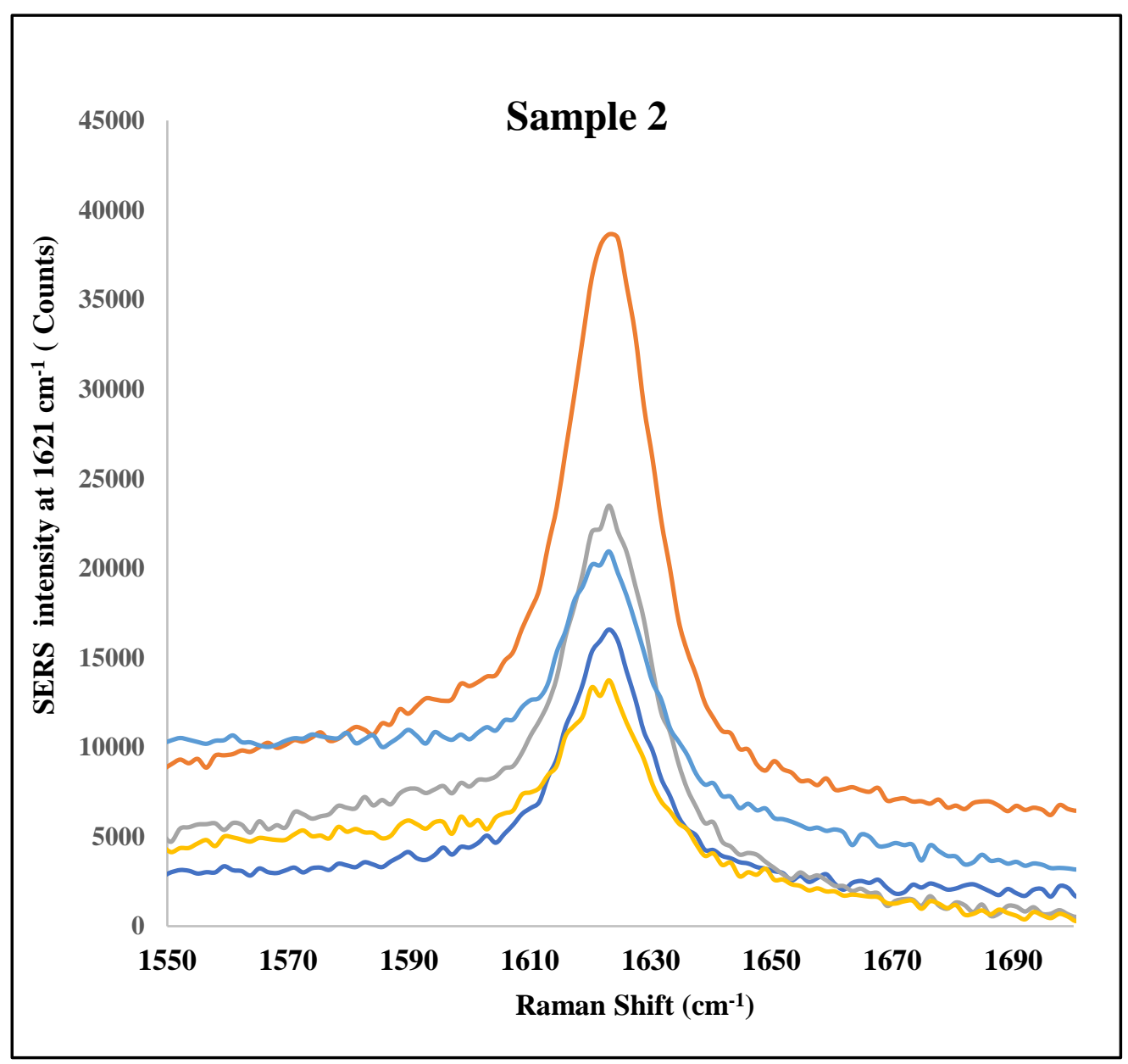

Figure C8: SERS intensity of $10^{-4} \mathrm{M} \mathrm{MB}$ at $1621 \mathrm{~cm}^{-1}$ from 5 different spots of 3D nanoprism superlattices (sample 2). 


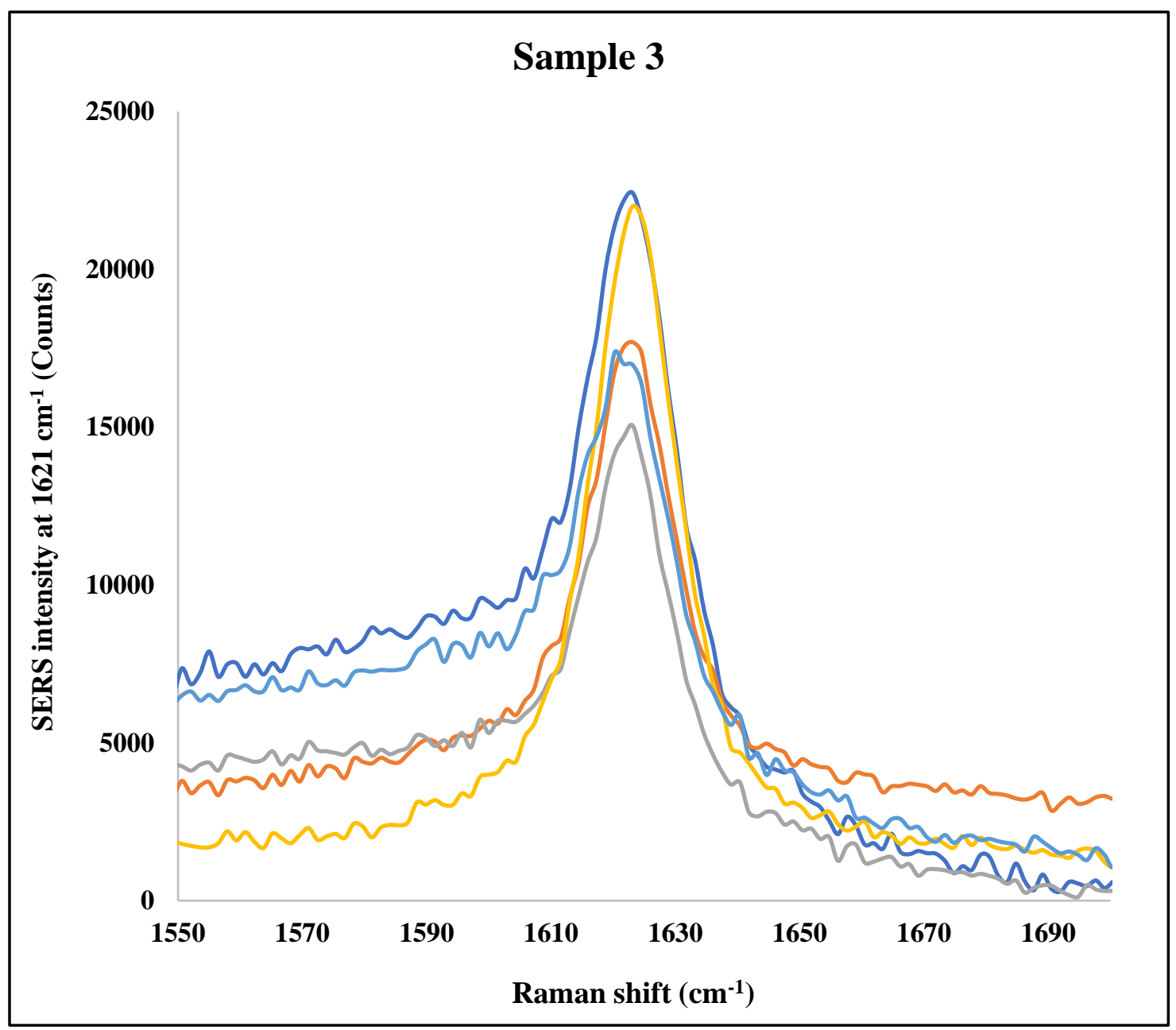

Figure C9: SERS intensity of $10^{-4} \mathrm{M} \mathrm{MB}$ at $1621 \mathrm{~cm}^{-1}$ from 5 different spots of 3D nanoprism superlattices (sample 3). 


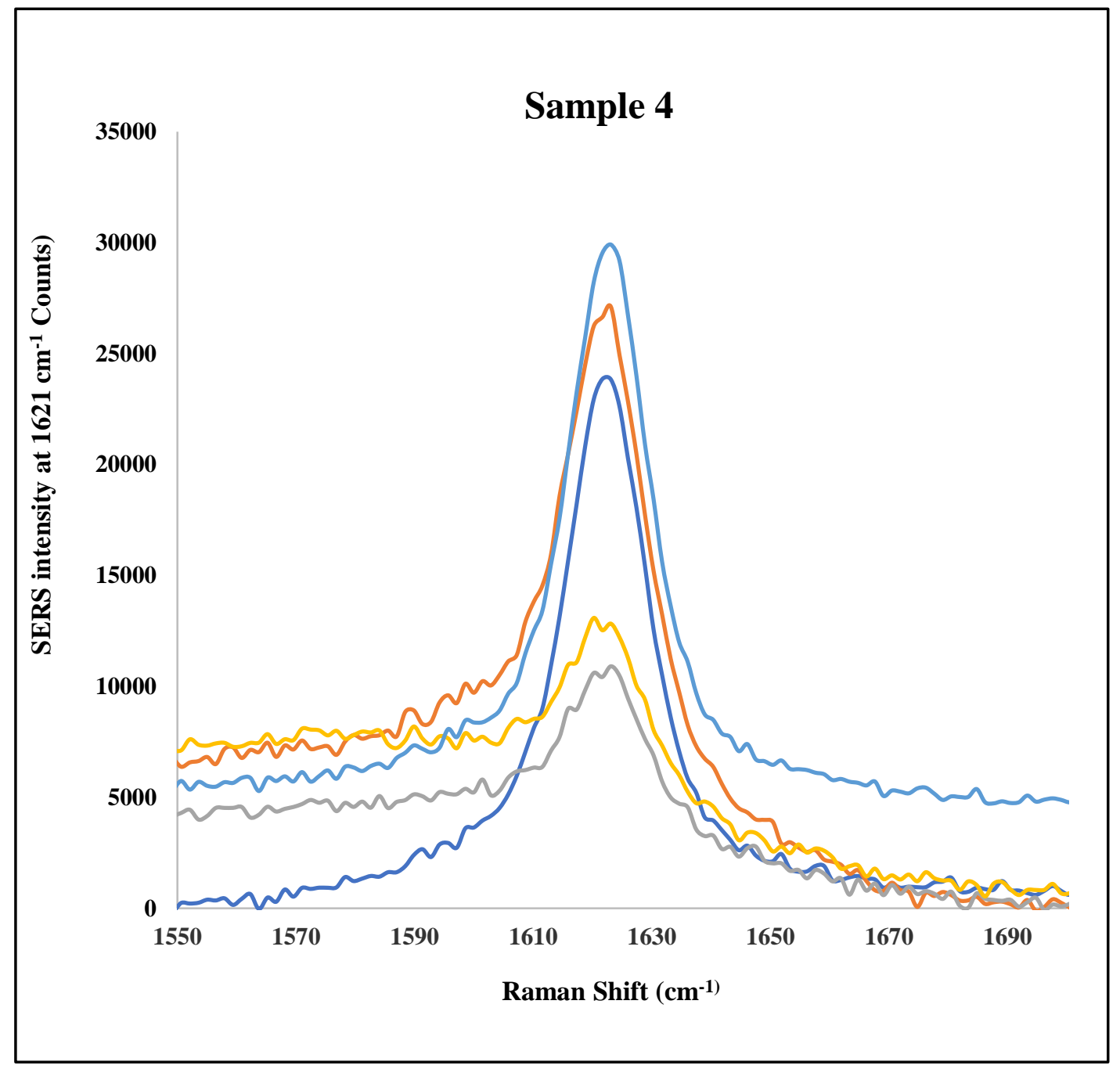

Figure C10: SERS intensity of $10^{-4} \mathrm{M} \mathrm{MB}$ at $1621 \mathrm{~cm}^{-1}$ from 5 different spots of 3D nanoprism superlattices (sample 4). 


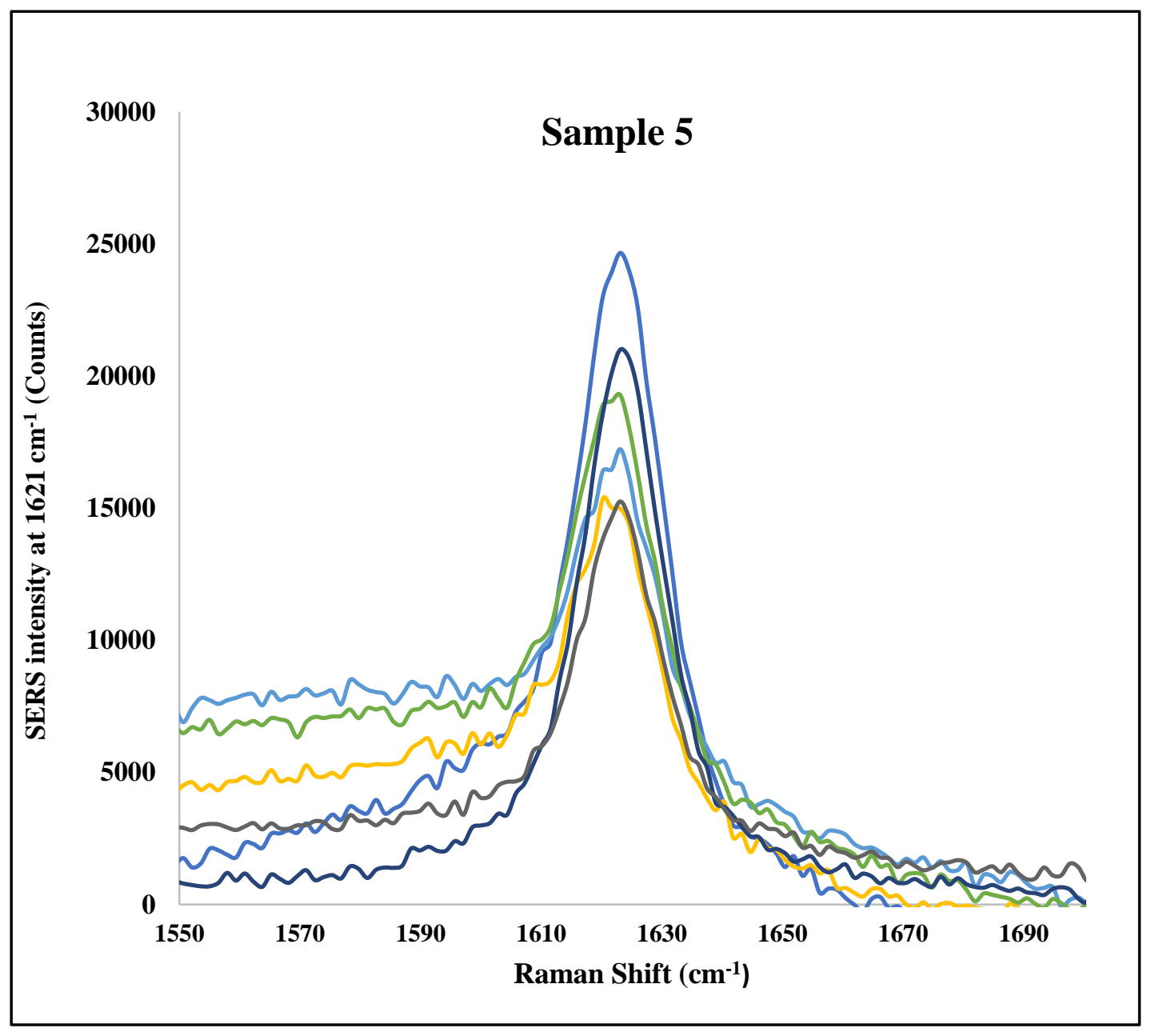

Figure C11: SERS intensity of $10^{-4} \mathrm{M}$ MB at $1621 \mathrm{~cm}^{-1}$ from 5 different spots of 3D nanoprism superlattices (sample 5). 
Table C1: Statistical reproducibility analysis of SERS performance of 3D nanoprisms superlattice

\begin{tabular}{cc}
\hline Range of SERS intensity (a.u.) & Frequency (counts) \\
$10000-15000$ & 6 \\
$20000-25000$ & 8 \\
$15000-20000$ & 8 \\
$25000-30000$ & 2 \\
$<30000$ & 1 \\
\hline
\end{tabular}




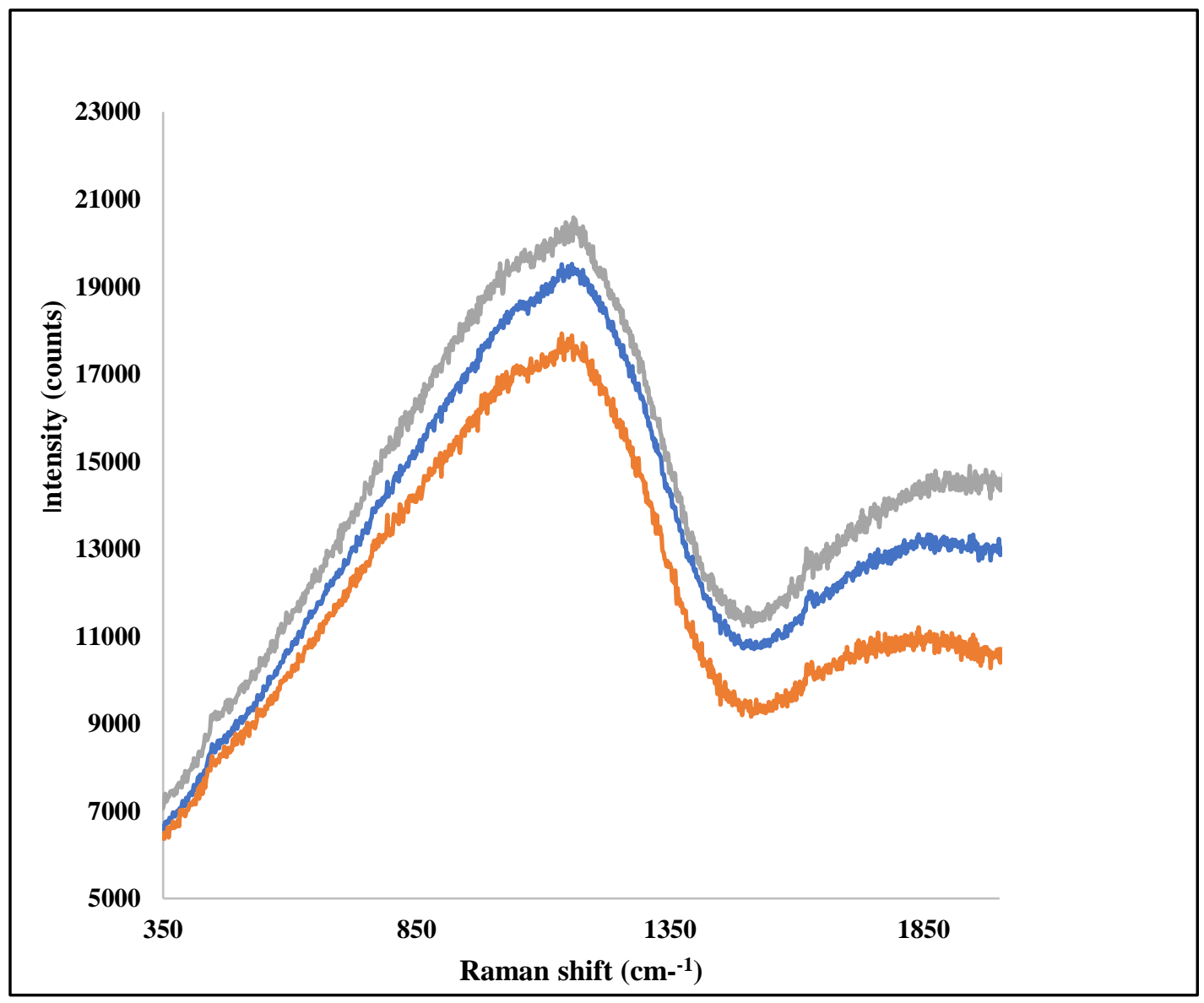

Figure C12: Additional SERS spectra of $10^{-4} \mathrm{M}$ MB on bare PS beads 


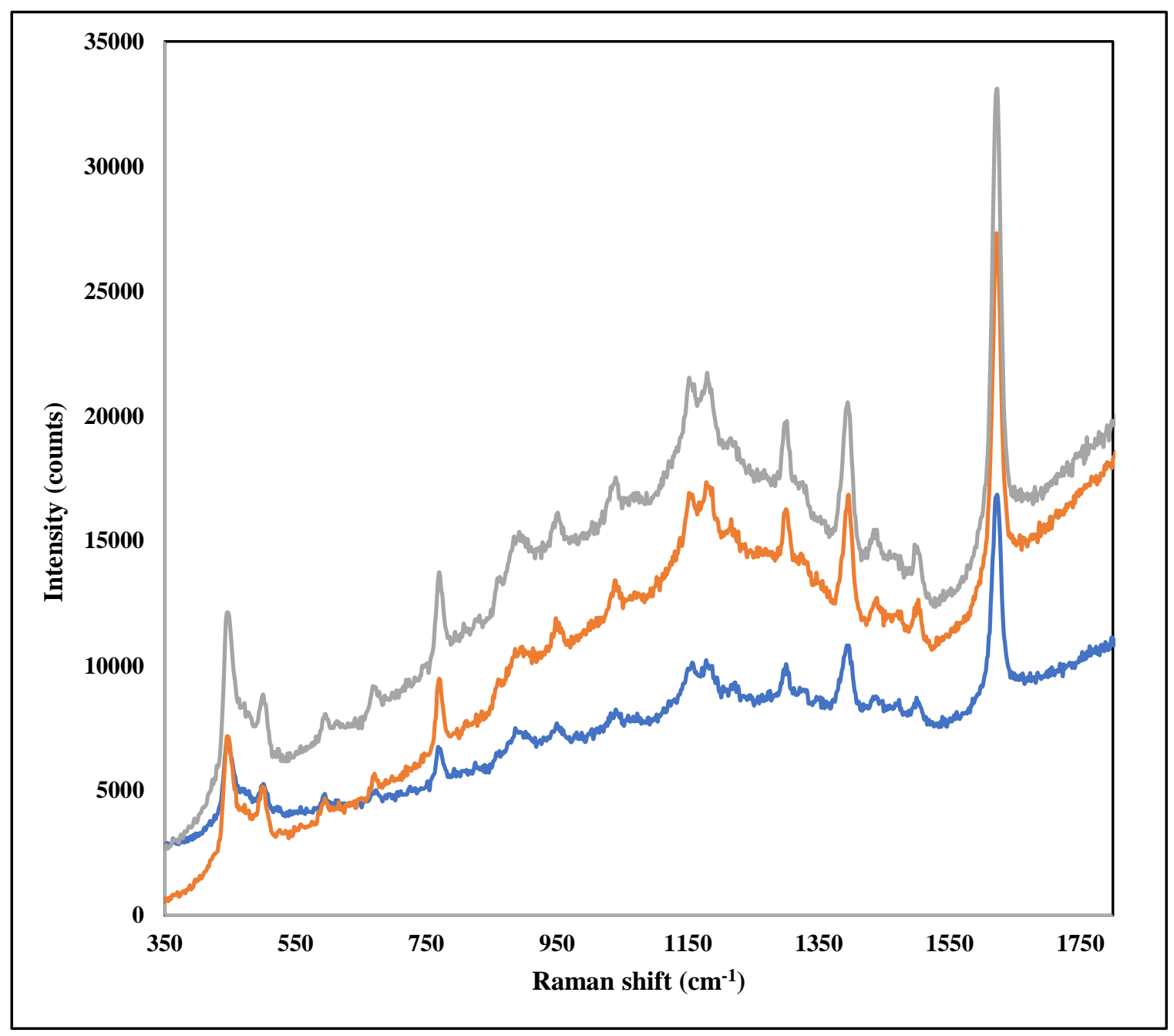

Figure C13: Additional SERS spectra of $10^{-6} \mathrm{M}$ MB on 3D PS bead/nanoprism aggregates. 


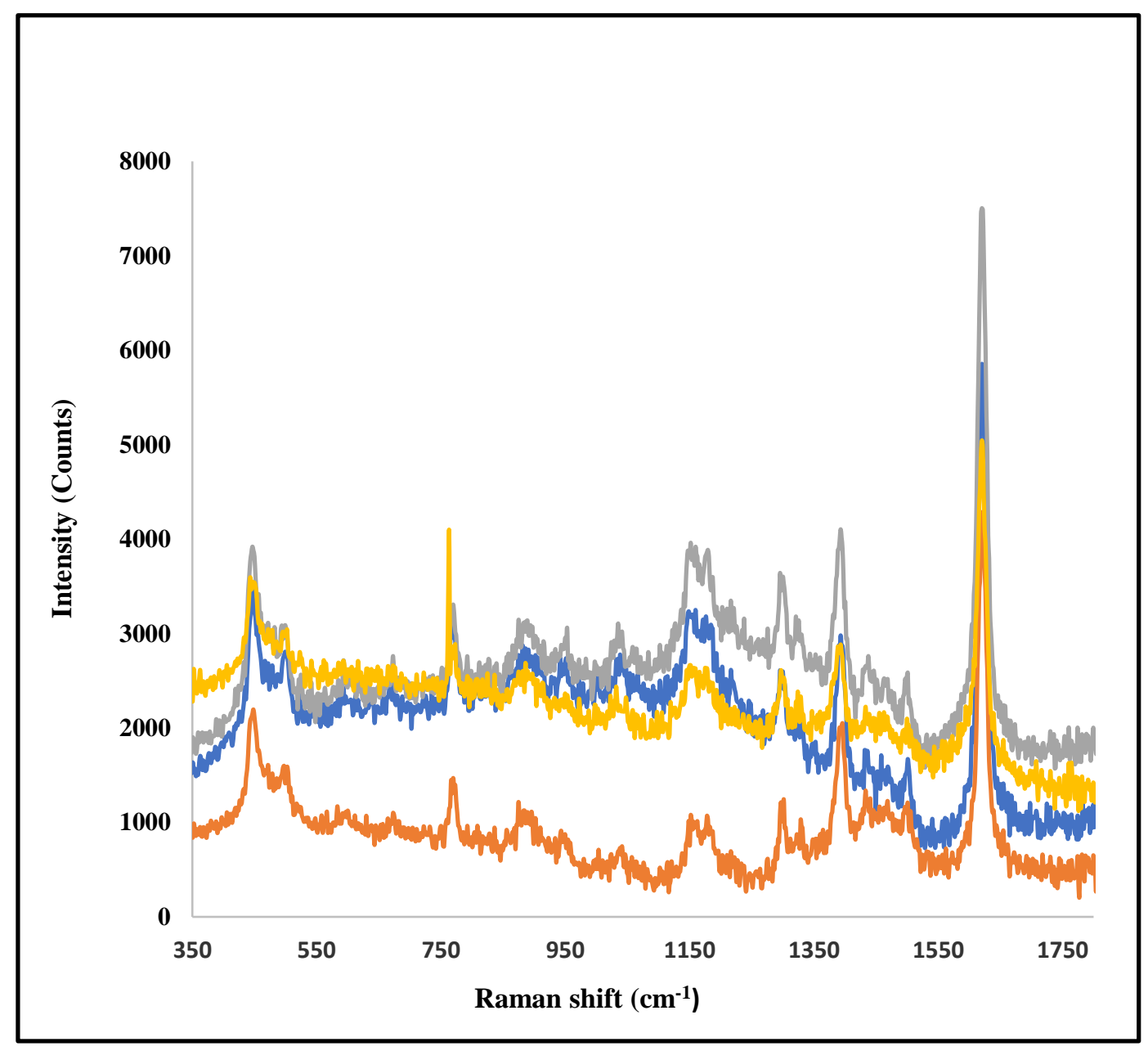

Figure C14: Additional SERS spectra of $10^{-7} \mathrm{M} \mathrm{MB}$ on 3D PS bead /nanoprismsaggregates. 


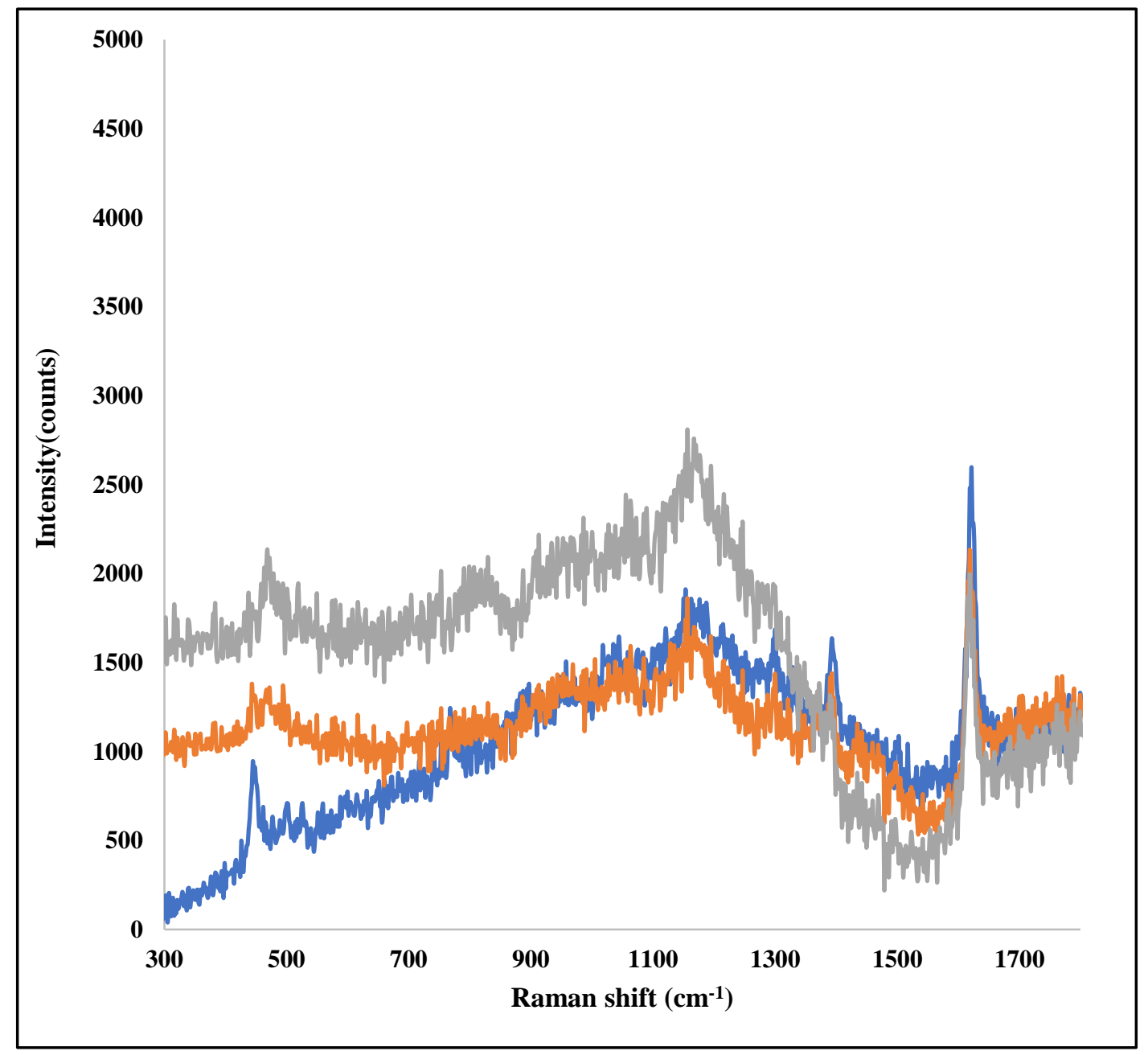

Figure C15: Additional SERS spectra of $10^{-8} \mathrm{M} \mathrm{MB}$ on 3D PS bead /nanoprism aggregates. 


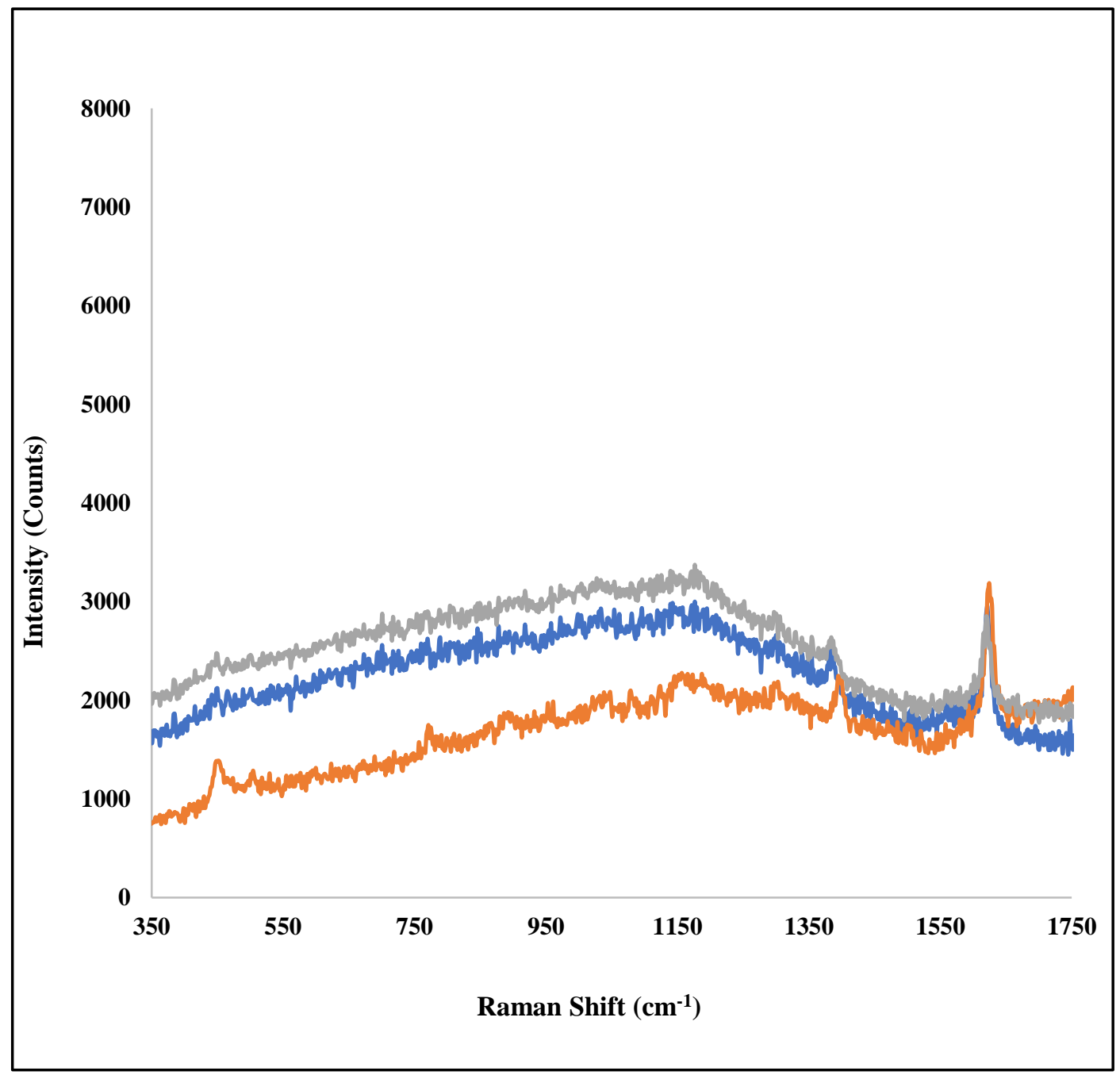

Figure C16: Additional SERS spectra of $10^{-9} \mathrm{M}$ MB on 3D PS bead /nanoprism aggregates. 


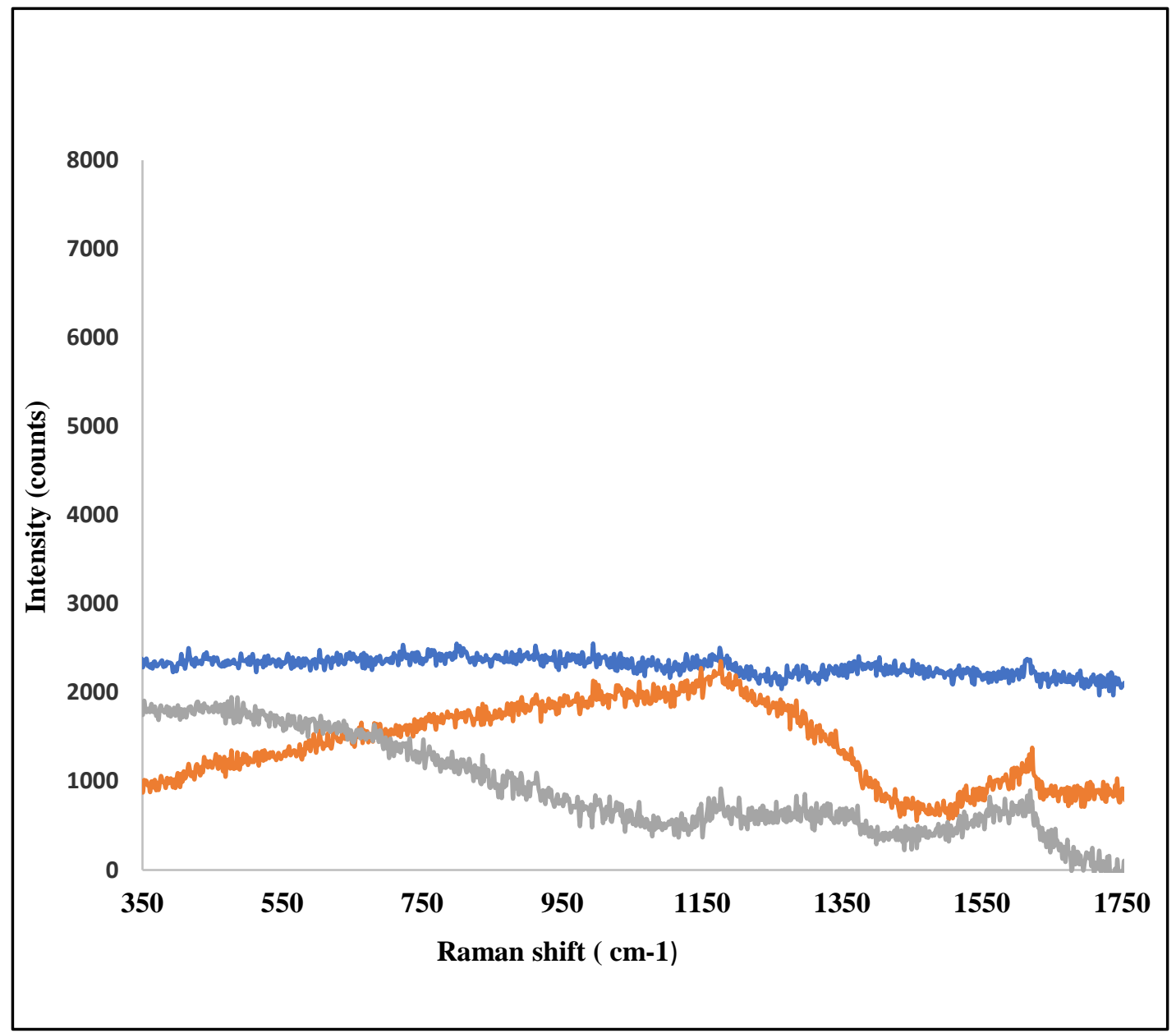

Figure C17: Additional SERS spectra of $10^{-10} \mathrm{M}$ MB on 3D PS bead /nanoprism aggregates. 


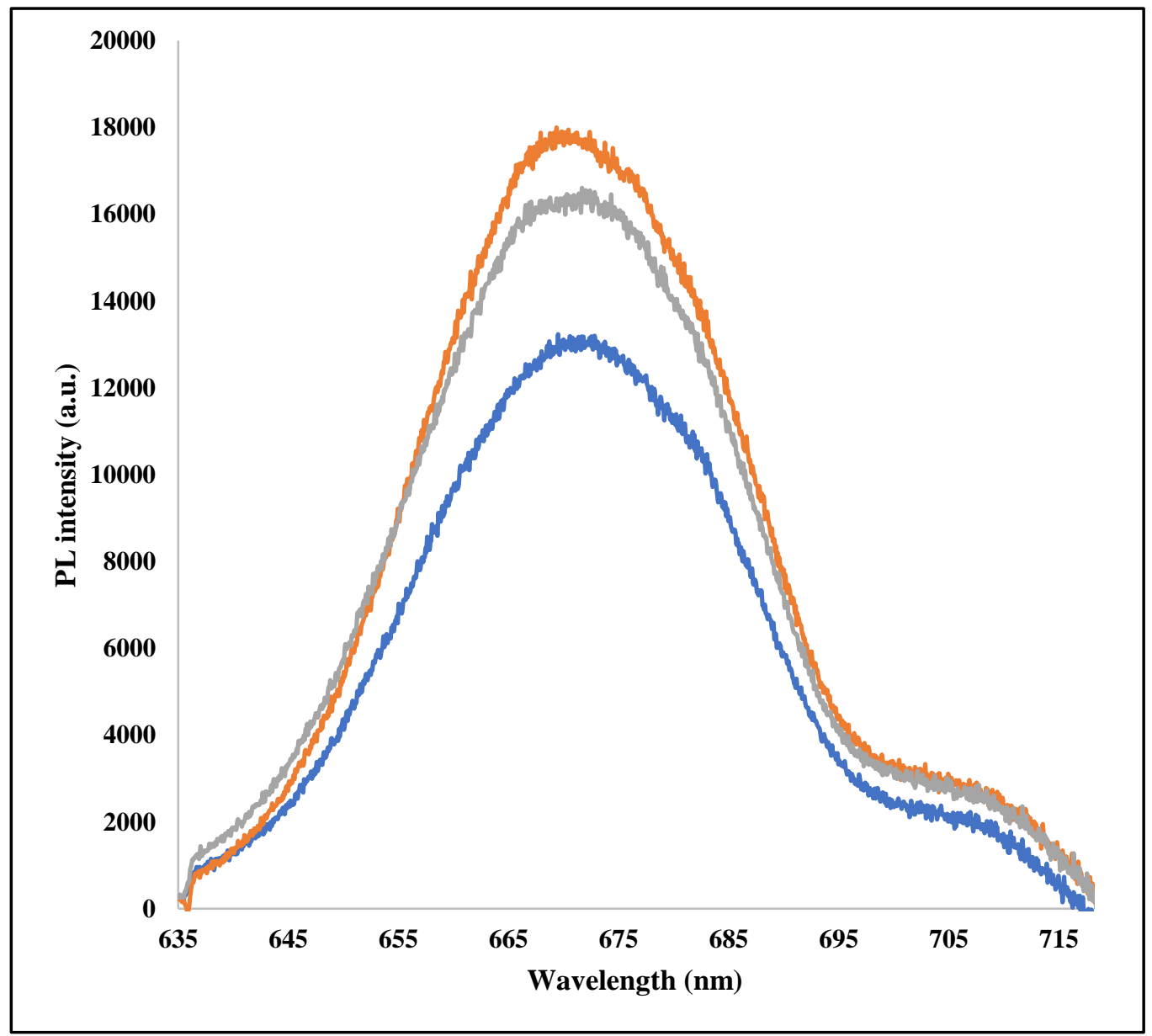

Figure C18: Additional PL spectra of Alexa Fluor Phalloidin 647 using 3D nanoprism superlattices. 


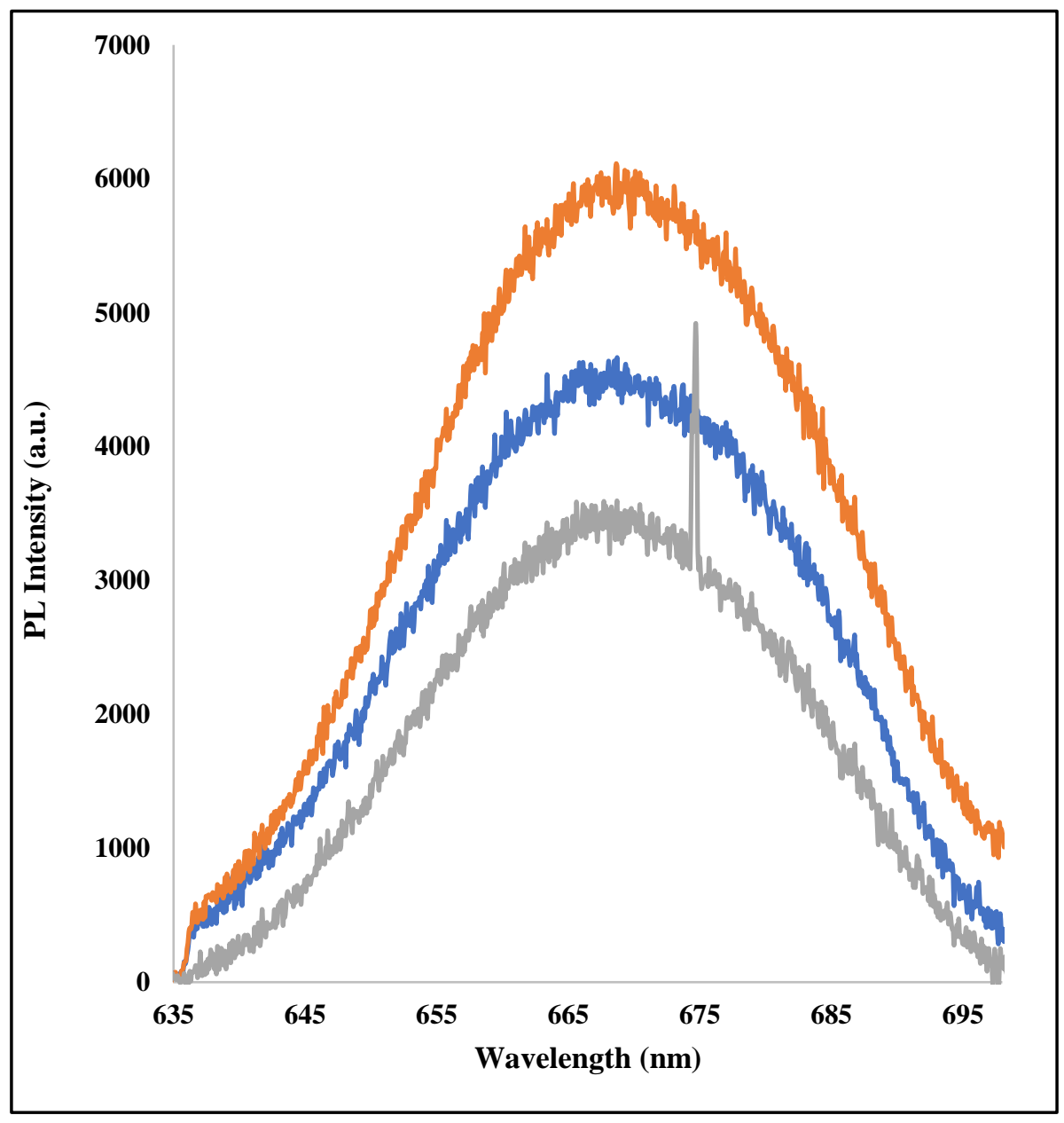

Figure C19: Additional PL spectra of Alexa Fluor Phalloidin 647 on pristine glass slides (blank). 


\title{
CURRRICULUM VITAE
}

\author{
Md. Emtias Chowdhury \\ 216 West Collins Court, Louisville, KY 40214 \\ 502-936-4198 | m0chow01@ louisville.edu
}

\section{SUMMARY}

Qualified by a research background in the synthesis and characterization of nanoparticle-biomolecule conjugates and formulation and directed assembly of nanoparticles and biopolymer composites using a diverse set of laboratory skills and instrumentation techniques. Core competencies include:

- Bioconjugation of DNA / Nucleic acid to nanoparticles and polymer beads.

- Extensive hands-on experience in an array of analytical and surface characterization instruments.

- Characterization of nanomaterials' surface functionalization, formulation, and bioconjugation.

- Familiarity with spherical nucleic acids, and polymeric particles for drug delivery.

- Detail oriented, multitasking, and strong interpersonal and communication skills.

- Ability to work independently or in a team environment 


\section{SKILLS}

- Instrumentation techniques: Dynamic light scattering, UV-Vis spectroscopy, Plate reader, Gel electrophoresis, Darkfield, Brightfield \& Fluorescence microscopy imaging, Photoluminescence, Raman scattering, FT-IR, SEM, AFM, STEM, Mass spectrometry, HPLC, GC-MS, XRD, NMR.

- Experimental skills: Surface modification of nanoparticles, polymer beads and glass substrate, Carbodiimide coupling, Formulation, Spray drying, Organic synthesis, Surface chemistry, Assay development (Oligreen ss-DNA assay, BCA assay, Antioxidant, and scavenging assay), Nanoparticle-biomolecule conjugates, and Analytical separation.

- Computer skills: MS office, R, ImageJ, Igor Pro.

\section{RESEARCH EXPERIENCE}

University of Louisville Louisville, KY

PhD Researcher $12 / 2014$ to current

- Synthesized high yield (around 55\%) nanoparticle homo- and hetero-dimers through DNA directed assembly of Janus nanocrystals for plasmonic biosensing.

- Worked on the optimization of curcumin- chitosan spray dried formulation leading to improvement in size distribution and efficacy of curcumin encapsulated particles by $30 \%$.

- Optimized crystallization pathways to minimize 'kinetic trapping' effect in DNAinduced hierarchical assembly of nanoprisms $(\sim 80 \mathrm{~nm})$ into microscale nanoparticle superlattice.

- Studied plasmonic properties of 3D arrays of gold nanocrystals that exhibited significant surface-enhanced Raman scattering $\left(<10^{6}\right)$ and fluorescence (up to 10 times) enhancement.

- Synthesized polymer bead and nanoparticle composite plasmonic substrate via DNA interaction for ultra-low $(20 \mathrm{pg} / \mathrm{mL})$ SERS detection of methylene blue. 
- Improved reliability and accuracy of compound identification by $20 \%$ in GC-MS analysis of complex bio-oil mixture using retention index constraint along with mass spectral matching.

- Identified and quantified Phenolics (21.7 wt.\%) in Lignin Fast pyrolysis bio-oils by GC-MS analysis to assess the feasibility of using oil extract as complete replacements for phenol in resins.

\section{LEADERSHIP EXPERIENCE}

University of Louisville

Louisville, KY

Teaching Assistant 08/2014 to current

- Led multiple laboratory sections of CHEM-207 and 208 class consisting of 30-35 students.

- Led exam proctoring for CHEM-202 class comprising 250-300 students and mentored 3 undergrad students on nanoparticle synthesis, characterization, and DNA functionalization.

\section{EDUCATION}

University of Louisville Louisville, KY

Doctor of Philosophy in Chemistry $11 / 2020$ (Expected)

GPA: $3.74 / 4.00$

University of Maine

Orono, ME

Master of Science in Analytical chemistry

08/2014

GPA: $3.83 / 4.00$

University of Chittagong

Chittagong, Bangladesh

Bachelor of Science in Chemistry

06/2010

GPA: $3.18 / 4.00$ 


\section{PUBLICATION | SEMINAR | POSTER}

- Anushka E Vithanage, Emtias Chowdhury, Luz D Alejo, Paige C Pomeroy, William J DeSisto, Brian G Frederick, William M Gramlich. "Renewably sourced phenolic resins from lignin bio- oil.” J. Appl. Polym. Sci. 2017,134, 44827.

- Shahinur Rahaman, khoa Phung, Anwar Hossain, Emtias Chowdhury, Martin O'toole, Jacek B. Jasinski, Noppadon sathitsuksanoh. "Hydrophobic functionalization of HY zeolites for efficient conversion of glycerol to solketal.” Applied Catalysis A: General 2020, 592, 117369.

- Emtias Chowdhury, Craig Grapperhaus, Martin O’Toole. "Facet selective asymmetric functionalization of nanoprisms for Janus particle synthesis." J Nanopart Res 2020, 22,1-12.

- Emtias Chowdhury, Craig A Grapperhaus, Martin G. O’Toole. "DNA induced assembly of gold nanoprisms and polymer beads into 3D plasmonic SERS substrate." Nanotechnology 2020, 32, 2.

- Emtias Chowdhury, Craig A Grapperhaus, Martin G. O’Toole. "DNA mediated hierarchical organization of gold nanoprisms into 3D superlattice for surface enhanced fluorescence and Raman scattering.” Journal of Materials Chemistry C (In Revision)

- Emtias Chowdhury, Craig A Grapperhaus, Martin G. O’Toole. "High yield synthesis and Fano-type resonance of gold nanoprisms dimers and trimers. (Manuscript in prep.)

- Emtias Chowdhury, Martin G. O'Toole. "DNA mediated 3D hierarchical organization of gold nanoprisms". KY Nano/AM Symposium, 08/2019, Louisville, KY.

- Emtias Chowdhury, Martin G. O’Toole. "Synthesis of Janus gold nanoprisms and high yield nanoprism dimers in solution." ACS conference and regional meeting, 04/2019, Orlando, Florida.

- Emtias Chowdhury, Martin G. O’Toole. “Amphiphilic Janus gold nanoplates for Supramolecular assembly.” 2018 KY Nano/AM Symposium, 08/2018, Louisville, KY.

- Emtias Chowdhury, Martin G. O’Toole. "Dual Coated Amphiphilic Gold Nanoplates for Supramolecular Assembly." Society of Biomaterials annual meeting, 08/2017, Atlanta, GA. 


\section{AWARDS}

- Winner (Most awesome category) of KY multi-scale Nano Image contest, 2020.

- Dissertation completion award, University of Louisville, 2020.

\section{VOLUNTARY EXPERIENCE}

- Volunteered in organizing science demonstrations for INSPIRES programs in 2016 and 2018. 